\title{
Ionization signal measurement through detection of electroluminescent light
}

\author{
by
}

\section{James Lacey}

\author{
A thesis submitted to the \\ Faculty of Graduate Studies and Research \\ in partial fulfilment of the requirements \\ for the degree of \\ Master of Science \\ Ottawa-Carleton Institute for Physics \\ Department of Physics \\ Carleton University \\ Ottawa, Ontario \\ January 2011 \\ (C)James Lacey, 2010
}




\begin{tabular}{|c|c|}
\hline $\begin{array}{l}\text { Library and Archives } \\
\text { Canada }\end{array}$ & $\begin{array}{l}\text { Bibliotheque et } \\
\text { Archives Canada }\end{array}$ \\
\hline $\begin{array}{l}\text { Published Heritage } \\
\text { Branch }\end{array}$ & $\begin{array}{l}\text { Direction du } \\
\text { Patrimoine de l'édition }\end{array}$ \\
\hline $\begin{array}{l}395 \text { Wellington Street } \\
\text { Ottawa ON K1A ON4 } \\
\text { Canada }\end{array}$ & $\begin{array}{l}\text { 395, rue Wellington } \\
\text { Ottawa ON K1A ON4 } \\
\text { Canada }\end{array}$ \\
\hline
\end{tabular}

Your file Votre référence

ISBN: 978-0-494-79606-1

Our file Notre référence

ISBN: 978-0-494-79606-1

NOTICE:

The author has granted a nonexclusive license allowing Library and Archives Canada to reproduce, publish, archive, preserve, conserve, communicate to the public by telecommunication or on the Internet, loan, distribute and sell theses worldwide, for commercial or noncommercial purposes, in microform, paper, electronic and/or any other formats.

The author retains copyright ownership and moral rights in this thesis. Neither the thesis nor substantial extracts from it may be printed or otherwise reproduced without the author's permission.
AVIS:

L'auteur a accordé une licence non exclusive permettant à la Bibliothèque et Archives Canada de reproduire, publier, archiver, sauvegarder, conserver, transmettre au public par télécommunication ou par l'Internet, prêter, distribuer et vendre des thèses partout dans le monde, à des fins commerciales ou autres, sur support microforme, papier, électronique et/ou autres formats.

L'auteur conserve la propriété du droit d'auteur et des droits moraux qui protège cette thèse. Ni la thèse ni des extraits substantiels de celle-ci ne doivent être imprimés ou autrement reproduits sans son autorisation.
In compliance with the Canadian Privacy Act some supporting forms may have been removed from this thesis.

While these forms may be included in the document page count, their removal does not represent any loss of content from the thesis.
Conformément à la loi canadienne sur la protection de la vie privée, quelques formulaires secondaires ont été enlevés de cette thèse.

Bien que ces formulaires aient inclus dans la pagination, il n'y aura aucun contenu manquant.

\section{Canadä}




\section{Abstract}

In the search for neutrinoless double beta decay, a new generation of experiments is being developed. The EXO collaboration is developing a gas phase xenon electroluminescence (EL) time projection chamber to search for the decay in ${ }^{136} \mathrm{Xe}$. A key requirement in the development of such a detector is exceptional energy resolution, on the order of $1 \%$ or better at the Q-value for xenon. EXO proposes to utilize electroluminescence optical gain for signal amplification, where the energy will be measured indirectly from the resulting light signals. This work investigates EL optical gain in xenon and the achievable resolution for $5.49 \mathrm{MeV}$ $\alpha$ particles, measured using a cylindrical EL counter operated as a gas proportional scintillation counter without gas gain. The gain and resolution were measured as a function of applied drift field, optical gain amplification and threshold parameters were determined from a fit to the data, resulting in an amplification of $A=119.9 \pm 0.280($ stat $) \pm 8.7($ sys $) \quad$ (photons $/ \mathrm{kV}$ ) and threshold of $B=0.882 \pm 0.0017($ stat $) \pm 0.0206(s y s) \quad(k V / c m-b a r)$. The resolution was measured as a function of voltage and an energy resolution on the order of $0.8 \%$ was be demonstrated. 


\section{Acknowledgements}

I would like to acknowledge my supervisor, Dr. Kevin Graham for his support and guidance throughout the course of my MSc, as well as Dr. Cliff Hargrove, whos insight and lab experience were invaluable in the completion of the research. I would also thank Philippe Gravelle and Yves Baribeau for all their help and advice throughout the course of the project. Finally I would like to thank the entire Carleton EXO group for helping make the last two and a half years of study extremely rewarding and enjoyable. 


\section{Contents}

Abstract $\quad$ ii

Acknowledgements $\quad$ iii

List of Tables $\quad$ vi

List of Figures vii

1 Introduction $\quad \mathbf{1}$

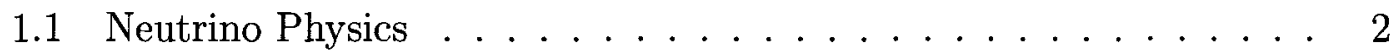

1.1.1 Neutrino Oscillations . . . . . . . . . . . . . 4

1.1 .2 Double beta decay ................. 13

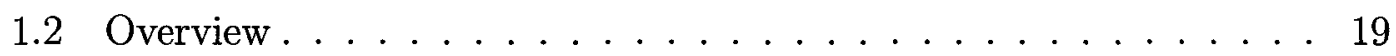

2 EXO: Experiment Background 21

2.1 Ionization \& Scintillation in Xenon ............. 25

2.2 Electroluminescence in Xenon . . . . . . . . . . . . . . . . 32

2.3 Enriched Xenon Observatory (EXO): Gas-phase . . . . . . . . . 35

2.3.1 XEP: A High Pressure Xe (HPXe) Electroluminescent TPC 37

2.4 Experimental Requirements .................. . . . . . . 41

2.4.1 Energy Resolution for $\beta \beta_{0 \nu} \ldots \ldots . \ldots . \ldots 41$

2.4 .2 Tracking for $\beta \beta_{0 \nu} \ldots \ldots \ldots . \ldots . \ldots 46$

2.4.3 Background suppression and rejection for $\beta \beta_{0 \nu} \ldots \ldots 48$

3 Electroluminescence (EL) Test Chamber $\quad 50$

3.1 Detector Design . . . . . . . . . . . . . . 56

3.1 .1 EL Insert Design ................ 60

3.1.2 PMT Response Characterization \& Calibration . . . . . . 66

3.2 Data Acquisition and Detector Output . . . . . . . . . . 72

4 Monte Carlo Chamber Simulation $\quad 78$ 
4.1 Shadowing \& Chamber Efficiency . . . . . . . . . . . . . 79

4.2 Ionization \& Scintillation Pulse Simulation . . . . . . . . . . . . 87

4.3 PMT Signal Simulation . . . . . . . . . . . . . . . 91

5 Data Analysis and Results $\quad 100$

5.1 PMT Baselines ... . . . . . . . . . . . . . . 103

5.2 Track Angle Cuts and Efficiency . . . . . . . . . . . . . 107

5.3 Run-time Signal Stability . . . . . . . . . . . . . . . 115

5.4 Response Stability During The Data Acquisition Period . . . . . . . 124

5.5 Anode signal analysis . . . . . . . . . . . . . . 126

5.6 Scintillation Signal . . . . . . . . . . . . . . . . . . . . . . . . . . . . . . . . . . . . . . . . . . . . .

5.7 EL Signal and Optical Gain . . . . . . . . . . . . . . . . 146

5.7 .1 EL resolution . . . . . . . . . . . . . . . 154

6 Discussion and Conclusions $\quad 161$

$\begin{array}{lr}\text { Bibliography } & 169\end{array}$ 


\section{List of Tables}

1.1 Family of Leptons . . . . . . . . . . . . . . . . 4

1.2 Family of Antileptons . . . . . . . . . . . . . . . . 4

2.1 Xenon properties table. . . . . . . . . . . . . 25

2.2 Literature $\mathrm{W}$-values. . . . . . . . . . . . . . . . . . 32

3.1 Detector dimensions $\ldots \ldots \ldots \ldots \ldots$

3.2 PMT gain. . . . . . . . . . . . . . 70 


\section{List of Figures}

1.1 Mass spectrum for three neutrino states [1]. . . . . . . . . . 12

1.2 Feynman diagram for two neutrino double beta decay. . . . . . . . 14

1.3 Feynman diagram for neutrinoless double beta decay. . . . . . . . . 14

1.4 Summed energy spectrum for the two outgoing electrons. Reprinted with permission from [2], API Copyright 2008. . . . . . . 15

1.5 Double beta decay mass as a function of the minimum neutrino mass. Reprinted with permission from [2], API Copyright 2008. . . 18

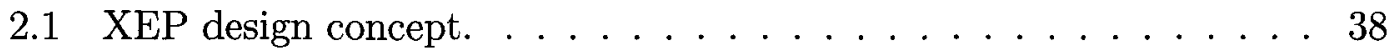

3.1 Electric field vs. radial position. . . . . . . . . . . . . . 52

3.2 Cylindrical EL detector concept. . . . . . . . . . . . . . . 52

3.3 Lesker T vacuum chamber. Drawing by M. Bowcock. . . . . . . . 57

3.4 EL chamber insert with Teflon cap and Teflon insulated high-voltage wire. . . . . . . . . . . . . . . . . 5 57

3.5 Drawing of cylindrical anode/cathode insert. Drawing by M. Bow-

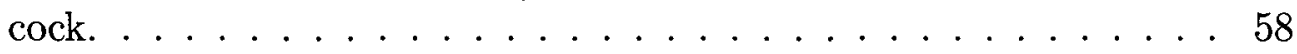

3.6 ADMCA spectrum of the ${ }^{241} \mathrm{Am}$ source. Lines indicate the region of interest, FWHM, and the centroid. . . . . . . . . . 59

3.7 Distribution of ionization track lengths for alpha particles in 1 bar of xenon obtained from GEANT4. . . . . . . . . . . . 6 62

3.8 Optical gain and $R_{E L}$ dependence on anode radius: top: a) EL threshold plotted using Equation (3.2) with $B=0.83(\mathrm{kV} / \mathrm{cm}-\mathrm{bar})$ [3], bottom: b) EL gain determined by integrating Equation (2.14) . 64

3.9 a) System setup used for PMT calibration.

b) Calibration circuit. . . . . . . . . . . . . . . 67

3.10 PMTB $1.3 \mathrm{kV}$ : ADMCA spectrum showing an SPE peak centered at channel 75 and a pulse generator peak located at channel $\approx 830 \quad 69$

3.11 SPE spectrum. . . . . . . . . . . . . . . . 70

3.12 PMT gain including systematic errors vs. PMT voltage: Top curve: PMTA, Bottom curve: PMTB . . . . . . . . . . . . . . 71 
3.13 Integrated scintillation amplitudes for PMT's A\&B. Gaussian fits result in mean values which agree within $3 \% \ldots \ldots \ldots . . . . .72$

3.14 Ionization track orientation $\theta \quad \& \quad \phi$ dependence. a) Side view of EL insert. b) Axial view of EL insert. . . . . . . . . . . . . 76

3.15 a) Perpendicular ionization track: Raw PMT signal shown in black. Raw anode signal shown in red. b) Parallel ionization track: Raw PMT signal shown in black. Raw anode signal shown in red. . . . . 77

3.16 Raw PMT traces illustrating angular dependence. . . . . . . . . . 77

4.1 GEANT representation of EL test chamber. The vacuum chamber walls and PMTs are not shown. Axis colours: Red $=\mathrm{x}$, Yellow=y, Blue $=$. . . . . . . . . . . . . . . . . . . . . . 8 80

4.2 a) Depiction of efficiency simulation. b) Conversion of the 2-D efficiency map into a 3-D efficiency distribution. . . . . . . . . . 81

4.3 Light collection efficiency distributions corresponding to the scintillation region. Obtained with and without the EL insert. . . . . . 83

4.4 Light collection efficiency distributions corresponding to the EL region. Obtained with and without the EL insert. . . . . . . . . 83

4.5 Anode shadowing distributions for the scintillation and EL regions. 84

4.6 Sum PMT photon hit efficiency as a function of y-position for selected z-positions. . . . . . . . . . . . . . 85

4.7 Sum PMT photon hit efficiency as a function of z-position for selected y-positions. . . . . . . . . . . . . 86

4.8 2-D efficiency map of the $\mathrm{y}-\mathrm{z}$ plane for $(0.51<y<5.95) \mathrm{cm}$ and

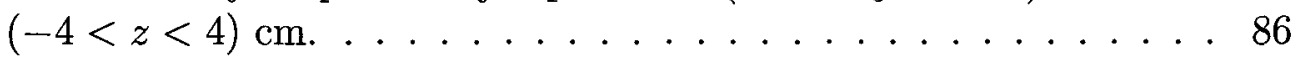

$4.9 \alpha$ ionization track produced in GEANT4 . . . . . . . . 88

$4.10 \mathrm{dE} / \mathrm{dx}$ corresponding to the ionization track of Figure 4.9. $\ldots . .88$

4.11 Scintillation pulse corresponding to the $\alpha$ event of Figure $4.9 \ldots 89$

4.12 GEANT4 result for the number of expected photons detected by the PMTs assuming $W_{s}=34.3 \mathrm{eV} . \ldots \ldots \ldots \ldots$

4.13 GEANT4 result for the number of expected photons detected by the PMTs assuming $W_{s}=49.6 \mathrm{eV} \ldots \ldots \ldots \ldots$

$4.143 \mathrm{kV}$ simulated PMT trace corresponding to the ionization track of

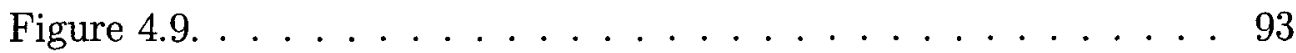

$4.153 \mathrm{kV}$ : Simulated PMT trace comparison with data: perpendicular $\alpha$ track . . . . . . . . . . . . . . . . . . 94

4.16 Comparison of real scintillation pulse to the pulse generated by GEANT4. . . . . . . . . . . . . . . . . . 994

$4.173 \mathrm{kV}$ simulated EL pulse comparison with data for a perpendicular $\alpha$ track. . . . . . . . . . . . . . . 95 
$4.183 \mathrm{kV}$ simulated EL pulse comparison with data for a parallel $\alpha$ track. 95

$4.193 \mathrm{kV}$ simulated EL pulse for a perpendicular $\alpha$ track: comparison with data after rebinning. . . . . . . . . . . . . . 97

4.20 MC/data $6 \mathrm{kV}$ PMT trace comparison for a selection of $\alpha$ track angles. . . . . . . . . . . . . . . . . . . . . . 99

4.21 MC/data $3 \mathrm{kV}$ PMT trace comparison for a selection of $\alpha$ track angles. . . . . . . . . . . . . . . . . . . . 99

5.1 Raw PMT signal illustrating the trace characteristics. . . . . . . . 101

5.2 PMT baselines obtained from fit: 1) baseline fit over region $t=$ $0 \rightarrow t=t_{0}-3 \mu \mathrm{s}$. 2) baseline fit over region $t=95 \mu \mathrm{s} \rightarrow t=100 \mu \mathrm{s} 104$

5.3 PMT average baselines over the region $t=0 \rightarrow t=t_{0}-3 \mu s: 1$ ) raw distribution of data. 2) distribution of data excluding points outside $3 \sigma \ldots \ldots \ldots \ldots$. . . . . . . . . . . . . . . . . . . . . . . .

5.4 Baseline distributions obtained for voltages of 2,4 , and $6 \mathrm{kV} . \quad \ldots 105$

5.5 Baseline mean values vs. voltage. . . . . . . . . . . . 106

5.6 Baseline RMS vs. voltage. . . . . . . . . . . . . . . 107

5.7 Exponential fit to the leading edge of a perpendicular $\alpha$ track. . . . 108

5.8 Signal and EL onset drift times: measured values and simulated results . . . . . . . . . . . . . . . . . 110

5.9 Average drift velocities for drift to the EL region and for total charge collection: measured values \& simulated results . . . . . . . . . 111

5.10 Ratio of the measured to simulated average drift velocities. . . . . . 112

5.11 a) EL vs. EL onset time distribution. b) The corresponding onset time distribution showing the timing cuts (30 in total). Red signifies the cut start and blue the cut end. c) $\theta$ ranges corresponding to the timing cuts in b. . . . . . . . . . . . . 114

5.12 Efficiency of the scintillation and EL regions as a function of $\theta$-cut for $6 \mathrm{kV} \ldots \ldots \ldots \ldots \ldots$. . . . . . . . . . . . . . . . . . . . . . . . . .

5.13 EL region efficiency vs. voltage: Angular dependence of EL efficiency for selected $\theta$-ranges. . . . . . . . . . . . . . . . . . . . . 117

5.14 Temperatures measured at 3 representative positions in the lab. The time period is $\approx 16 \mathrm{~h}:$

Bottom two curves PMTA \& PMTB, Top curve: Power supply rack 118

5.15 EL, scintillation, and anode signals vs. event number. Red: temperature, Black: Signal . . . . . . . . . . . . . 118

5.16 1.) The mean of every 500 events for the EL signal 2.) The RMS of every 500 events for the EL signal 3.) RMS/mean ratio . . . . . 119

5.17 EL distribution corrected for temperature variations. . . . . . . . 120 
$5.186 \mathrm{kV}$ EL distributions obtained over various event ranges before and after temperature corrections. . . . . . . . . . . . . . . 121

$5.196 \mathrm{kV}$ scintillation distributions obtained over various event ranges after temperature corrections. . . . . . . . . . . . . . 122

$5.206 \mathrm{kV}$ anode amplitude distributions obtained over various event ranges after temperature corrections. . . . . . . . . . . . . 123

5.21 Scintillation spectrum obtained with ADMCA setup. Fit with a skewed Gaussian convoluted with a linear background function. . . 125

5.22 SPE and scintillation Gaussian means, and the total number of detected scintillation photons per event vs. day: Measurements obtained using the ADMCA setup. Systematic errors are presented. 126

5.23 Anode amplitude vs. EL onset time. . . . . . . . . . . . . . 127

5.24 Gaussian fit to a $6 \mathrm{kV}$ anode amplitude distribution for the angular range: $20^{\circ}<\theta<30^{\circ} \ldots \ldots$. . . . . . . . . . . . 129

5.25 Anode amplitude vs. voltage for selected $\theta$-ranges showing angular dependence. Statistical errors are included but are not visible on the given scale. . . . . . . . . . . . . . . . . . . . . . . 129

5.26 Linear fit applied to the anode amplitude vs. onset time. . . . . . 130

5.27 Anode amplitude corrected for angular dependence vs. voltage for selected $\theta$-ranges. The angular dependence is now removed. . . . . . 130

5.28 Anode amplitude vs. voltage: raw amplitude and decay time corrected amplitude. Statistical errors are included but are not visible on the given scale. . . . . . . . . . . . . . . . 132

5.29 Number of ionization electrons collected. Constant fit applied above $1.5 \mathrm{kV}$ resulting in $\approx 228000$ electrons. . . . . . . . . . . . . . . 132

5.30 Resolution of the ionization signal for a selection of angular range. . 133

5.31 Gaussian fit to the scintillation distribution obtained at $2 \mathrm{kV}$. . . 135

5.32 Scintillation mean vs. voltage for the angular range $\left(20^{\circ}<\right.$ theta $<$ $30^{\circ}$ ). Statistical errors are included but are not visible on the given scale. . . . . . . . . . . . . . . . . . 138

5.33 Scintillation values as a function of voltage corresponding to the two integration time windows for a selection of angular ranges. Statistical errors are included but are not visible on the given scale. . . . 139

5.34 Average scintillation value taken over the voltage range (0-5) $\mathrm{kV}$ as a function of angular range $(\theta$-cut $) . \ldots . . . . . . . .140$

5.35 Scintillation signal resolution vs. voltage. . . . . . . . . . . . 141

5.36 Scintillation distributions obtained at voltages of $5 \mathrm{kV}$ and $6 \mathrm{kV} \ldots .142$ 
5.37 Plots of the scintillation resolution as a function of $\theta$-cut number for voltages of 2,4 , and $6 \mathrm{kV}$. The presented errors are statistical but there is correlation between neighbouring points due to overlap in the angular ranges. . . . . . . . . . . . . . . . . 143

5.38 Scintillation resolution vs voltage corresponding to the two given $\theta$-ranges. . . . . . . . . . . . . . . . . . . . 144

5.39 Comparison of temperature corrected and uncorrected scintillation resolutions. . . . . . . . . . . . . . . . . . . . . 144

$5.406 .5 \mathrm{kV}$ : distribution of integrated EL values. . . . . . . . . 146

$5.41 \mathrm{EL}$ values vs. voltage corresponding to the given angular ranges. Statistical errors are included but are not visible on the given scale. 148

$5.42 \mathrm{EL}$ values vs. voltage for a selection of angular ranges with the angular dependence removed. Statistical errors are included but are not visible on the given scale. . . . . . . . . . . . . . 148

5.43 Event by event EL gain vs. event number: Temperature induced signal variations are cancelled out. . . . . . . . . . . . . . . 149

$5.44 \mathrm{EL}$ optical gain $\left(45^{\circ}<\theta<50^{\circ}\right)$ : Comparison of $\mathrm{W}$-value corrections without efficiency averaging. Statistical errors are included but not visible on the given scale. . . . . . . . . . . . . . 150

$5.45 W_{s}=49.6 \mathrm{eV}$ with efficiency averaging: Gain curves corresponding to the first and last $\theta$-ranges. Statistical errors are included but not visible on the given scale. . . . . . . . . . . . . . . 152

$5.46 W_{s}=49.6 \mathrm{eV}:$ EL amplification and threshold parameters vs. angular range $(\theta$-cut). The presented errors are statistical but there is correlation between neighbouring points due to overlap in the angular ranges. . . . . . . . . . . . . . . . . 152

5.47 EL value vs. scintillation value. . . . . . . . . . 153

$5.48 W_{s}=49.6 \mathrm{eV}:\left(45^{\circ}<\theta<50^{\circ}\right)$ : EL optical gain including systematic errors introduced by the $\mathrm{W}$-value and efficiency scaling. . . . . 154

5.49 Temperature corrected and uncorrected EL distributions obtained at $6.5 \mathrm{kV}\left(20^{\circ}<\theta<30^{\circ}\right) \ldots \ldots \ldots \ldots \ldots \ldots \ldots$

5.50 EL resolution for temperature corrected and uncorrected signals compared to the resolution expected from counting statistics. . . . . 156

5.51 Temperature corrected EL resolution vs. $\theta$-cut for the selected voltages 2,4 , and $6 \mathrm{kV} . \triangle$ : temp corrected, $\nabla:$ uncorrected. The presented errors are statistical but there is correlation between neighbouring points due to overlap in the angular ranges. . . . . . . . 158

5.52 Temperature corrected EL resolutions for the voltage range (4.5-7.5)

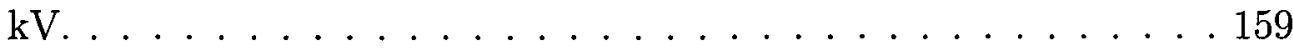


5.53 Energy resolution extrapolation curve obtained by taking $0.8 \%$ as the energy resolution at $5.49 \mathrm{MeV} \ldots \ldots \ldots \ldots$

6.1 EL resolution as a function of voltage including systematic errors due to integration windowing and baseline subtraction. . . . . . . 167 


\section{Chapter 1}

\section{Introduction}

This thesis describes research preformed on ionization signal measurements through the production and detection of electroluminescence light for the EXO collaboration towards the development of a high-pressure xenon gas time projection chamber. The Enriched Xenon Observatory (EXO) is an experimental particle physics collaboration dedicated to the measurement of the double beta decay half-life of ${ }^{136} \mathrm{Xe}$, and to investigate the neutrino mass spectrum through the study of neutrinoless double beta decay. EXO proposes to use an electroluminescence (EL) based time projection chamber in order to meet the experimental requirements needed for the identification of neutrinoless double beta decay events in ${ }^{136} \mathrm{Xe}$ and measurement of neutrino mass properties. The present work investigates the properties of electroluminescent light produced by $\alpha$ particles in a cylindrical drift 
chamber operated as a Gas Proportional Scintillation Counter (GPSC) without gas gain.

A cylindrical electroluminescence detector was constructed where the aim was to optimize the energy resolution obtained from measurements of the electroluminescent signal as well as to perform a detailed investigation of the electroluminescent light production and optical gain as a function of applied drift field. Energy resolution as a function of applied voltage for the ionization, scintillation and electroluminescence signals is measured, where a resolution on the order of $0.8 \%$ will be demonstrated for the EL signal. The EL optical gain was measured and the amplification and threshold parameters obtained from a fit to an existing model.

\subsection{Neutrino Physics}

The neutrino was first proposed in 1930 by Pauli as an explanation of the seemingly missing energy in measurements of the electrons emitted in nuclear $\beta$ decay [4]. The electron energy spectrum was found to be continuous and with a mean value well below the predicted energy which should be released in the decay. With the introduction of a new particle - the neutrino, Pauli was able to explain the continuous spectrum by assuming that in the process of nuclear $\beta$ decay a neutrino 
is emitted together with the electron in such a way that the sum of their energy is constant. Sensitive measurements of the energy and momentum of the electrons and the recoiling nucleus in $\beta$ decay were made which showed that a large portion of the energy and momentum were missing, therefore requiring the introduction of a new particle, the neutrino.

Following these observations, in 1932, Enrico Fermi postulated a theoretical framework for nuclear $\beta$ decay which included the neutrino [4]. However, the neutrino was not detected until much later, in 1957, utilizing a flux of anti-neutrinos $(\bar{\nu})$ from the Savannah River nuclear reactor [5]. The first observation of the electron antineutrino was made from the inverse $\beta$ reaction $\bar{\nu}_{e}+\mathrm{p}->\mathrm{e}^{+}+\mathrm{n}[5]$. Since the neutrino postulate of Pauli in 1930, it has been experimentally established that there are three light neutrinos, each one associated with the flavor of the corresponding leptonic interaction. The family of leptons is given in Table 1.1. There are three generations (flavours) of charged lepton, the electron, the muon, and the tau, and the corresponding generations of neutral leptons, the electron neutrino, the muon neutrino and the tau neutrino. There is also the family of antileptons where each particle is replaced with its antiparticle. Antiparticles are defined as the having the same mass but opposite values for other quantum numbers such as charge, parity, spin, and direction of magnetic moment. The interaction of a particle and antiparticle results in their annihilation with the production of radiant energy. Dirac fermions have distinct antiparticles while Majorana fermions 


\begin{tabular}{|c|c|c|}
\hline \multicolumn{3}{|c|}{ Family of Leptons } \\
\hline$e^{-}$ & $\mu^{-}$ & $\tau^{-}$ \\
$\nu_{e}$ & $\nu_{\mu}$ & $\nu_{\tau}$ \\
\hline
\end{tabular}

TABLE 1.1: Family of Leptons

\begin{tabular}{|c|c|c|}
\hline \multicolumn{3}{|c|}{ Family of Antileptons } \\
\hline$e^{+}$ & $\mu^{+}$ & $\tau^{+}$ \\
$\overline{\nu_{e}}$ & $\overline{\nu_{\mu}}$ & $\overline{\nu_{\tau}}$ \\
\hline
\end{tabular}

TABLE 1.2: Family of Antileptons

are identical to their antiparticles and determining which model applies to the neutrino is of fundamental importance for understanding the origin of neutrino masses and underlying symmetries of weak interactions [2].

\subsubsection{Neutrino Oscillations}

The idea that neutrinos oscillate between the three known flavours has been extensively verified in a large number of experiments [5-8]. The first measurement of the flux of neutrinos coming from the sun produced a result that disagreed with solar model calculated predictions by a factor of two. One way to account for the discrepancy was the introduction of neutrino flavour change, an idea first proposed by Pontecorvo. Proposed by Bethe and Critchfield, the solar model predicts that the generation of energy in the deep interior of the Sun is dominated by the $\mathrm{pp}$ 
fusion reaction.

$$
p+p \quad \rightarrow \quad{ }^{2} H+e^{+}+\nu_{e}
$$

To measure the solar neutrino flux Ray Davis operated a chlorine detector in the Homestake gold mine of Lead, South Dakota, which used the following reaction to determine the rate.

$$
{ }^{37} \mathrm{Cl}+\nu_{e} \quad \rightarrow \quad{ }^{37} \mathrm{Ar}+\mathrm{e}^{-}
$$

If neutrinos oscillate between flavours before they reach the detector then the number of $\nu_{e}$ available to interact with the $\mathrm{Cl}$ will be smaller thereby reducing the measured flux compared to that predicted, in agreement with the data [5]. The experiment was only sensitive to electron neutrinos and these are the only neutrinos expected to be produced in the Sun according to the solar model.

Oscillations between flavour states can occur if the physical neutrinos are a superposition of some number of independent mass states. A massive neutrino initially in a pure flavor state will oscillate between the possible flavor states as it propagates through space-time (flavour mixing). The observation of neutrino mixing is direct evidence for neutrinos being massive particles. In the general N-flavor case, if we consider neutrino flavour mixing in vacuum, a neutrino of a particular flavor $L$ can be represented in terms of a superposition of mass eigenstates as 
follows [2].

$$
\nu_{l L}=\sum_{\jmath=1}^{N} U_{l j} \nu_{\jmath L}, \quad l=e, \mu, \tau
$$

Here $\nu_{l L}$ are the neutrino flavor fields, $U$ is the Pontecorvo-Maki-Nakagawa-Sakata (PMNS) mixing matrix $U \equiv U_{P M N S}$, and $\nu_{j L}$ is the field of neutrino $\nu_{j}$ having a mass $m_{j}$. For three active neutrino flavours, $U$ takes the form of a $3 \times 3$ matrix with four free parameters.

$$
U=\left[\begin{array}{ccc}
U_{e 1} & U_{e 2} & U_{e 3} \\
U_{\mu 1} & U_{\mu 2} & U_{\mu 3} \\
U_{\tau 1} & U_{\tau 2} & U_{\tau 3}
\end{array}\right]
$$

Therefore there are three mass eigenstates 1,2 , and 3 , corresponding to the three masses $m_{1}, m_{2}, m_{3}$. This assumes no sterile neutrinos, ie. neutrinos which do not participate in interactions via any of the fundamental forces of the Standard Model except gravity.

$U$ can be parametrized by 3 angles and either 1 complex phase, if neutrinos are Dirac particles, or 3 complex phases, if neutrinos are Majorana particles. Dirac particles are distinct from their antiparticles while Majorana particles are identical to their antiparticles. A standard parametrization $U_{P M N S}$ takes the form [9]: 


$$
U=\left[\begin{array}{ccc}
c_{12} c_{13} & s_{12} c_{13} & s_{13} \\
-s_{12} c_{23}-c_{12} s_{23} s_{13} e^{i \delta} & c_{12} c_{23}-s_{12} s_{23} s_{13} e^{i \delta} & s_{23} c_{13} e^{i \delta} \\
s_{12} s_{23}-c_{12} c_{23} s_{13} e^{i \delta} & -c_{12} s_{23}-s_{12} c_{23} s_{13} e^{i \delta} & c_{23} c_{13} e^{i \delta}
\end{array}\right]
$$

where $c_{i j}=\cos \theta_{i j}, s_{i \jmath}=\sin \theta_{i j}$, with $\theta_{i \jmath} \in\left[0, \frac{\pi}{2}\right], \delta \in[0,2 \pi]$ is the Dirac CPV phase and $\alpha_{12}, \alpha_{13}$ are the Majorana CPV phases [9]. For purposes of illustration and to simplify the mathematics, the number of neutrino flavor eigenstates can be restricted to two, $\nu_{l}$ and $\nu_{k}$ for mixing between two neutrino mass states, $l$ and $k$. In this case the PMNS matrix reduces to the form [10]:

$$
U=\left[\begin{array}{cc}
\cos \theta & \sin \theta \\
-\sin \theta & \cos \theta
\end{array}\right]
$$

Using this form of $U$ along with the time dependant form of equation 1.1 $\left(\nu_{l L}=\sum_{\jmath=1}^{2} U_{l j} \nu_{j L} e^{-i E_{j} t}\right)$ the survival probability of neutrinos in vacuum is given by,

$$
P\left(\nu_{l} \rightarrow \nu_{k}\right)=\sin ^{2} 2 \theta \sin ^{2}\left(1.27 \Delta m^{2} \frac{L}{E_{\nu}}\right)
$$


where $\Delta m^{2}=\left|m_{2}^{2}-m_{1}^{2}\right|$ is the mass squared difference in $\mathrm{eV}^{2}, L$ is the distance from neutrino production to the detector in $\mathrm{km}$, and $E_{\nu}$ is the neutrino energy in $\mathrm{GeV}$. The probability $P_{\nu_{l} \rightarrow \nu_{k}}$ depends on the experimentally determined ratio $\frac{L}{E_{\nu}}$ and three fundamental parameters of nature, the mixing angle $\theta, m_{1}$ and $m_{2}$ $\left(\Delta m^{2}\right)$, of which only two can be measured independently [5]. Equation (1.4) gives the probability that a neutrino of energy $E_{\nu}$ and initial flavor state $l$ will be found in flavor state $k$ after travelling a distance $L$ in vacuum. It also shows that if neutrino mass eigenstates are zero $\left(\Delta m^{2}\right)$ then the probability for oscillation between flavor states is also zero.

Strong evidence for neutrino oscillation was first obtained in the observation of atmospheric neutrinos produced in the decay of hadronic showers initiated in the Earth's atmosphere by cosmic rays. The neutrinos are produced from the decay of pions and muons via weak interactions involving $W^{ \pm}$bosons (charged current interaction). The distance the neutrinos travel varies from $10-10000 \mathrm{~km}$ depending on whether downward or upward trajectories are considered, with the energy of the neutrinos spanning a range of $0.5-5 \mathrm{GeV}$. At $1 \mathrm{GeV}$, the product of flux and charged-current interactions cross-section is at a maximum and the expected neutrino flux is $1 \mathrm{~cm}^{-2} \mathrm{~s}^{-1}$ [5]. Super-Kamioka Nucleon Decay Experiments (Super-Kamiokande) is a neutrino observatory located under Mount Kamioka near the city of Hida, Gifu Prefecture, Japan. Super-Kamiokande has 
measured the asymmetry of the atmospheric $\nu_{\mu}$ flux due to the up-down asymmetry of the neutrino path length along with the dependence on zenith angle, while for electron neutrinos the event rate was found to be independent of direction and solid angle $[11,12]$. The resulting data strongly favoured the $\nu_{\mu} \rightarrow \nu_{\tau}$ oscillation hypothesis, where zenith angle dependent neutrino flavor change is the most reasonable explanation of the measurements. Under the oscillation hypothesis the mixing angle $\theta_{23}$ was found to be almost maximal, $\sin \left(\theta_{23}\right)>0.92$, and a value of $\Delta\left|m_{\text {atm }}^{2}\right|=2.4_{-0.5}^{+0.6} \mathrm{eV}^{2}$ was obtained at the $90 \%$ confidence level (Ashie et al. 2005) [11].

Since the Homestake experiment there have been considerable efforts to further measure the neutrino flux reaching Earth from the Sun. The Sudbury Neutrino Observatory (SNO) have obtained sensitive measurements of the solar neutrino flux. SNO obtained direct evidence for neutrino flavour oscillation was obtained using a large spherical heavy water Cherenkov counter which was operated $\approx 2 \mathrm{~km}$ underground in nickle mines located in Ontario, Canada. SNO has provided a method to check solar model predictions and neutrino properties independently through the analysis of the three neutrino reactions $[7,8,13]$ :

$$
\begin{aligned}
& \text { (Charged Current) } \nu_{e}+d \rightarrow p+p+e^{-} . \\
& \text {(Neutral Current) } \nu_{x}+d \rightarrow p+n+e^{-} .
\end{aligned}
$$




$$
\text { (Elastic Scattering) } \nu_{x}+e^{-} \rightarrow n \nu_{x}+e^{-} .
$$

The presence of matter in the Sun and the Earth can enhance neutrino oscillations, predicting a modulation in the flux of electron neutrinos with zenith angle due to traversing different amounts of matter. The SNO data when combined with the data from other neutrino experiments are inconsistent with solar model predictions in the absence of oscillations. The combined data are consistent with the threeflavor Mikheyev-Smirnov-Wolfenstein (MWS) model of matter enhanced neutrino oscillations thus providing strong evidence in support of the oscillation hypothesis.

Along with the study of atmospheric and solar neutrinos another method for the study of neutrino particle properties is to utilize the neutrino flux generated in nuclear reactors. The fission reactions used in nuclear reactors are very well understood and therefore the neutrino flux from reactors is accurately known. The KamLAND experiment studied the flux of reactor $\bar{\nu}_{e}$ over a flux-averaged mean baseline of $180 \mathrm{~km}$ making KamLAND sensitive to the neutrino mixing associated with the large mixing angle (LMA) solution to the solar neutrino problem [14]. Using $1000 \mathrm{t}$ of liquid scintillator measurements of the interaction rate and energy spectrum of $\bar{\nu}_{e}$ were obtained from the inverse $\beta$ decay reaction $\bar{\nu}_{e}+p \rightarrow e^{+}+n$. After a 766.3 tonne-year live-time, KamLAND has published accurate measurements of the $\bar{\nu}_{e}$ flux and energy spectrum providing very clear evidence of oscillation of reactor antineutrinos. 
Since the first neutrino flux measurements of the Homestake experiment, the evidence for neutrino oscillations from solar, atmospheric and reactor measurements has been overwhelming. The measurements obtained from the oscillation experiments have established the flavor change hypothesis of mixing of massive neutrinos and allow the determination of the mass squared difference for solar neutrinos, $\Delta m_{\odot}^{2}$, the mass squared difference for atmospheric neutrinos, $\left|\Delta m_{a t m}^{2}\right|$, and their corresponding mixing angles, $\sin ^{2} \theta_{\odot}$ and $\sin ^{2} 2 \theta_{a t m}$ with relatively good precision and to place stringent limits on $\sin ^{2} \theta_{13}$. The measurements indicate that $\Delta m_{\odot}^{2}=\Delta m_{21}^{2} \sim 8.0_{-03}^{+0.4} \times 10^{-5} \mathrm{eV}^{2},\left|\Delta m_{\text {atm }}^{2}\right|=2.4_{-05}^{+0.6} \times 10^{-3} \mathrm{eV}^{2}$, $\sin ^{2} 2 \theta_{\odot}=0.86_{-0.04}^{+0.03}, \sin ^{2} 2 \theta_{a t m}=0.92$ and $\sin ^{2} \theta_{13}<0.19[2,9]$. While the experiments have shown that neutrinos have a non-zero mass, and have made accurate measurements of the mass difference squared the results are insensitive to the sign of $\Delta m_{a t m}^{2}$ which leaves the hierarchy of the neutrino mass spectrum unknown. For 3- $\nu$ mixing there are two possibilities, $\Delta m_{31(32)}^{2}>0$ or $\Delta m_{31(32)}^{2}<0$ which correspond to two types of mass spectrum [2]:

type1: normal hierarchy, $\quad m_{1}<m_{2}<m_{3}, \quad \Delta m_{\text {atm }}^{2}=\Delta m_{31}^{2}>0$ with $m_{2} \simeq \sqrt{\Delta m_{\odot}}$ and $m_{3} \simeq \sqrt{\left|\Delta m_{a t m}\right|}$, type2: inverted hierarchy, $\quad m_{3}<m_{1}<m_{2}, \quad \Delta m_{\text {atm }}^{2}=\Delta m_{32}^{2}<0$ with $m_{1,2} \simeq \sqrt{\left|\Delta m_{a t m}\right|}$. 
Shown in Figure 1.1 are the two possible hierarchies defined by the measurements of $\Delta m_{a t m}^{2}$ and $\Delta m_{\odot}^{2}$.

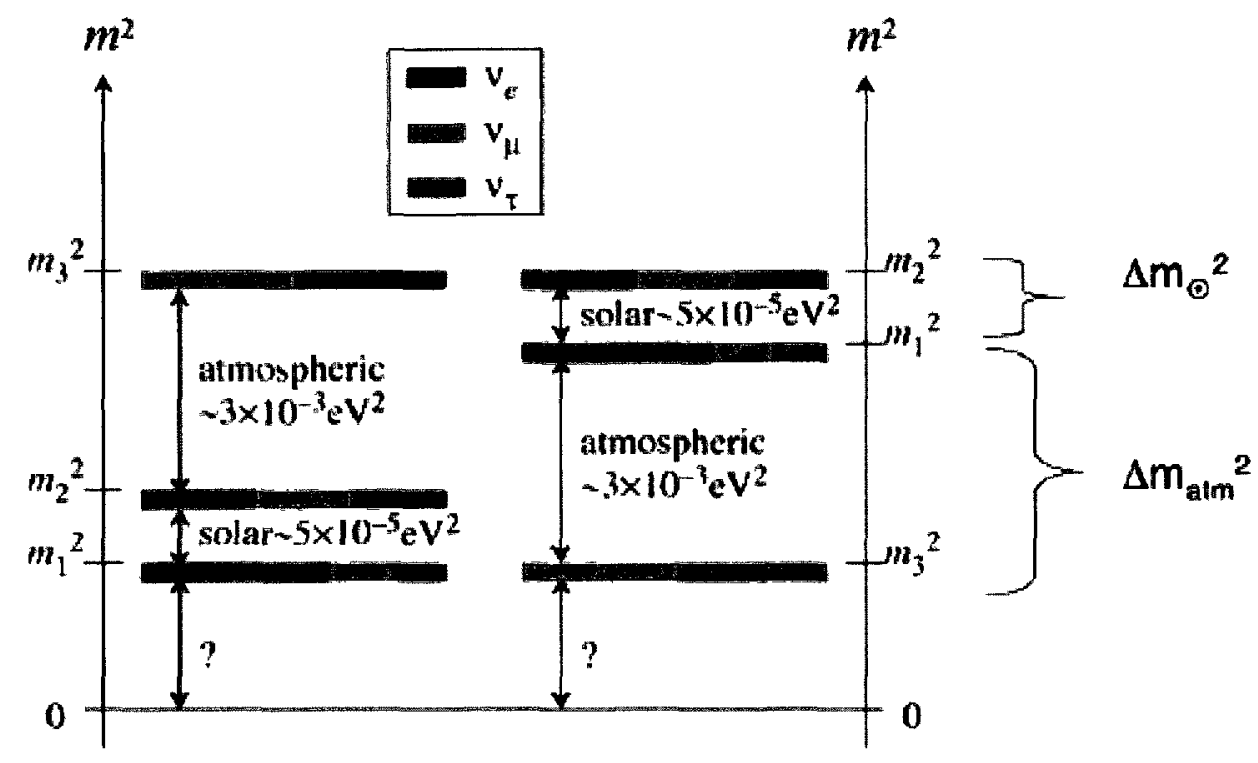

FIgURE 1.1: Mass spectrum for three neutrino states [1].

Oscillation experiments cannot probe the absolute mass scale of neutrinos and are completely insensitive to whether massive neutrinos are Dirac or Majorana by nature. The only experimental approach at present which seems feasible for establishing the Majorana nature of neutrinos are experiments searching for the extremely rare process of neutrinoless double beta decay $\left(\beta \beta_{0 \nu}\right)$ (see Section 1.1.2). The Standard Model (SM) of particle physics does not include neutrino flavor mixing and assumes that neutrinos are massless. However the observation of neutrino flavor oscillation through the mixing of mass states requires finite neutrino 
mass and therefore requires extensions to the existing Standard Model.

\subsubsection{Double beta decay}

Double beta decay is a process mediated by the weak interaction which can occur in nuclei which have an even number of protons and neutrons (even-even nucleus). In the decay an even-even nucleus with proton number $\mathrm{Z}$ and total nucleon number $\mathrm{A}$, $(Z, A)$, decays to the $(Z+2, A)$ state releasing energy, $Q_{\beta \beta}$, which is the difference in binding energy between the two nuclear states. Pairing forces make the parent nucleus more bound than the $(Z+1, A)$ nuclear state but less bound then the $(Z+2, A)$ nuclear state. In a 1935 paper by Maria Goeppert-Mayer [2], the author derived an expression for the rate of double beta decay with the emission of two electrons and two antineutrinos, and two years later a theory was developed by Ettore Majorana in which there is no distinction between $\nu$ and $\bar{\nu}$ [2]. The process of two neutrino double beta decay, $\left(\beta \beta_{2 \nu}\right)$, proceeds through a reaction of the form $(Z, A) \rightarrow(Z+2, A)+2 e^{-}+2 \overline{\nu_{e}}$. However if neutrinos are Majorana particles, the neutrinoless double beta decay process, $\left(\beta \beta_{0 \nu}\right)$, will occur in a reaction of the form $(Z, A) \rightarrow(Z+2, A)+2 e^{-}$, where the exchange of neutrino/antineutrino occurs as the exchange of a virtual particle (see Figures $1.2 \& 1.3$ ) [1].

The two decay processes, $\left(\beta \beta_{2 \nu}\right)$ and $\left(\beta \beta_{0 \nu}\right)$, have been the focus of many experimental searches beginning in 1948. A review of the experiments and their 


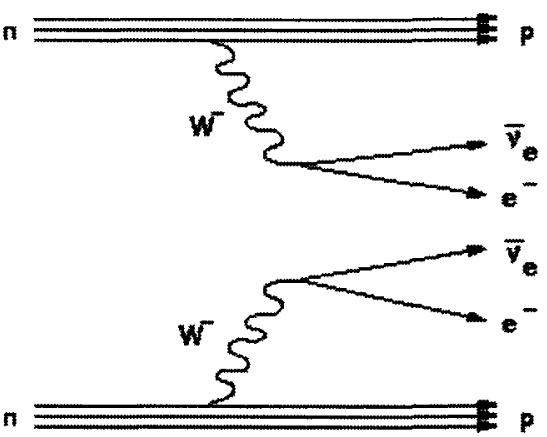

FIgURE 1.2: Feynman diagram for two neutrino double beta decay.

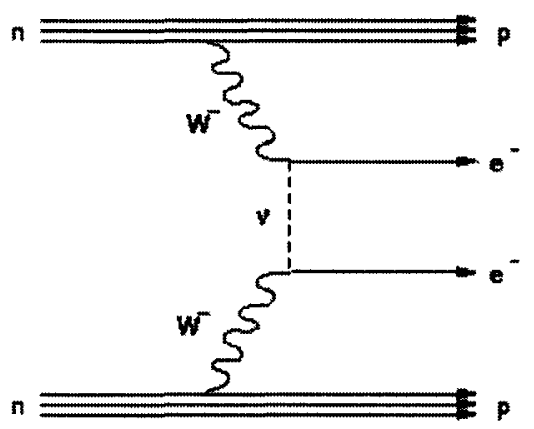

Figure 1.3: Feynman diagram for neutrinoless double beta decay.

results can be found in $[15,16]$. The two neutrino mode of double beta decay has been observed for a number of isotopes, and measurements of the half-lives and decay widths have been made. However to date there has not been an accepted observation of the neutrinoless mode of the decay, though there has been one recent claim of observation by a subset of the Heidelberg-Moscow Collaboration using ${ }^{76} \mathrm{Ge}$ (See [17]). Currently there are a large number of experiments running and under construction which are designed to further the search for neutrinoless double beta decay and reach sensitivities which will either confirm or refute the claim of observation.

In order to distinguish between the two processes an experimental signature must be available. As early as 1952, Primakoff had calculated the two electron summed energy spectrum and the electron-electron angular correlations [2], providing the means for distinguishing between the two decays [2]. For the $\beta \beta_{2 \nu}$ decay, the summed electron energy spectrum will be continuous, terminate at the 
$Q_{\beta \beta}$ endpoint, and have a peak value well below $Q_{\beta \beta}$. This is because the energy measured for the two electrons will depend on the amount of energy carried away by the two neutrinos. In the case of $\beta \beta_{0 \nu}$ the electron energy spectrum will be mono-energetic and peaked at $Q_{\beta \beta}$. Figure 1.4 shows the two different spectra.

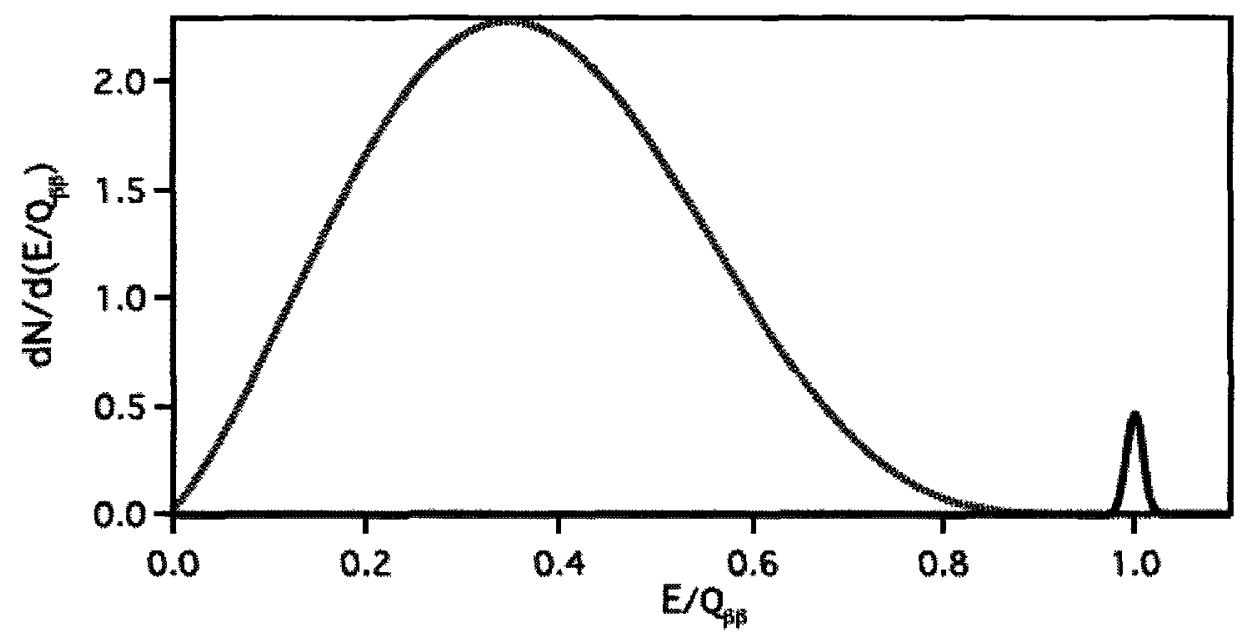

FiguRE 1.4: Summed energy spectrum for the two outgoing electrons. Reprinted with permission from [2], API Copyright 2008.

The decay rate for $\beta \beta_{2 \nu}$ can be expressed as [2],

$$
\left(T_{1 / 2}^{2 \nu}\right)^{-1}=G_{2 \nu}\left(Q_{\beta \beta}, Z\right)\left|M_{2 \nu}\right|^{2}
$$

where $G_{2 \nu}\left(Q_{\beta \beta}, Z\right)$ is the phase space factor for the emission of the two electrons and the two antineutrinos, and $M_{2 \nu}$ is the nuclear matrix element for this second order process. The $\beta \beta_{2 \nu}$ decay does not depend on the masses of the neutrinos, 
does not discriminate between Dirac and Majorana particles, and conserves lepton number [2]. Assuming the decay of $\beta \beta_{0 \nu}$ is mediated by the exchange of light neutrinos with left-handed interactions, the rate for the process can then be expressed as [2],

$$
\left(T_{1 / 2}^{0 \nu}\right)^{-1}=G_{0 \nu}\left(Q_{\beta \beta}, Z\right)\left|M_{0 \nu}\right|^{2}<m_{\beta \beta}>
$$

where $G_{0 \nu}\left(Q_{\beta \beta}, Z\right)$ is the phase space factor for the emission of the two electrons, $M_{0 \nu}$ is the corresponding matrix element, and $\left\langle m_{\beta \beta}\right\rangle$ is the effective Majorana neutrino mass, defined as [18]:

$$
<m_{\beta \beta}>=\left|\sum_{j} m_{j} U_{e j}^{2}\right|=\left.\left|m_{1}\right| U_{e 1}\right|^{2}+m_{2}\left|U_{e 2}\right|^{2} e^{i\left(\alpha_{2}-\alpha_{1}\right)}+\left|U_{e 3}\right|^{2} e^{i(-\alpha-2 \delta)} \mid,
$$

where the $m_{j}$ 's are the masses of the three neutrinos, $\delta$ is a "Dirac" phase analogous to the phase in the CKM matrix [18], and $\alpha_{1,2}$ are "Majorana" phases which only affect Majorana particles [18]. $U_{e j}$ are the top row components of the mixing matrix $U$ which allows one to write the well-defined mass states as a linear combination of flavor states [18]. The rate given by Equation (1.9) is for the $\beta \beta_{0 \nu}$ process when mediated by the exchange of light Majorana neutrinos with left-handed interactions. There are however other possible neutrinoless double 
decay mechanisms, such as the exchange of Goldstone bosons, and therefore the exchange of light Majorana neutrinos is considered to be the minimum mechanism. For a more detailed discussion of the possible mechanisms the reader is refered to the review of Avignone et al[2]. The positive identification of $\beta \beta_{0 \nu}$ requires new physics regardless of the mediating interaction.

In considering the double beta decay mass as a function of the lightest neutrino mass eigenstate, $\min \left(m_{j}\right)$, the plot shown in Figure 1.5 is obtained which shows the regions corresponding to the different mass orderings. The two regions correspond to inverted and normal hierarchies respectively. In principle $\min \left(m_{j}\right)$ can be measured in an extremely low-background beta decay experiment with ideal energy resolution, however this is not possible in practise due to technological constraints [2]. As can be easily seen from Figure 1.5, depending on the mass of the lightest neutrino, and on the sign of $\Delta m_{\text {atm }}^{2}$ the neutrino mass spectrum can take one of the following three forms [2]:

- Normal Hierarchical (NH):

$m_{1}<<m_{2}<<m_{3}$, with $m_{2} \cong \sqrt{\Delta m_{\odot}^{2}} \sim 0.009 \mathrm{eV}$, and $m_{3} \cong \sqrt{\Delta m_{\text {atm }}^{2}} \sim 0.047$

$\mathrm{eV} ; \bullet$ Inverted Hierarchical (IH):

$m_{3}<<m_{1}<m_{2}$, with $m_{1,2} \cong \sqrt{\Delta m_{a t m}^{2}} \sim 0.047 \mathrm{eV} ;$

- Quasi-Degenerate (QD):

with $m_{1} \cong m_{2} \cong m_{3} \cong m_{0}, \quad m_{j}^{2}>>\left|\Delta m_{\text {atm }}^{2}\right|, \quad m_{0} \geq 0.10 \mathrm{eV}$. 
Therefore a measurement of the $\beta \beta_{0 \nu}$ rate has the potential to distinguish between the mass hierarchies. However for some values of $m_{\beta \beta}$ and depending on the value of $\min \left(m_{\jmath}\right)$, the two regions overlap and hierarchy can not be determined. The observation of neutrinoless double beta decay would indicate the absolute neutrino mass, help decipher which mass hierarchy nature has chosen and also give insight into the underlying conservation laws of particles and their interactions. Lepton number $(\mathrm{L}), \mathrm{L}=+1$ for leptons and $\mathrm{L}=-1$ for antileptons, is generally regarded as a conserved quantity. A positive signal for $\beta \beta_{0 \nu}$ would indicate that $\mathrm{L}$ is not a conserved quantity since the decay requires $\Delta L=2$.

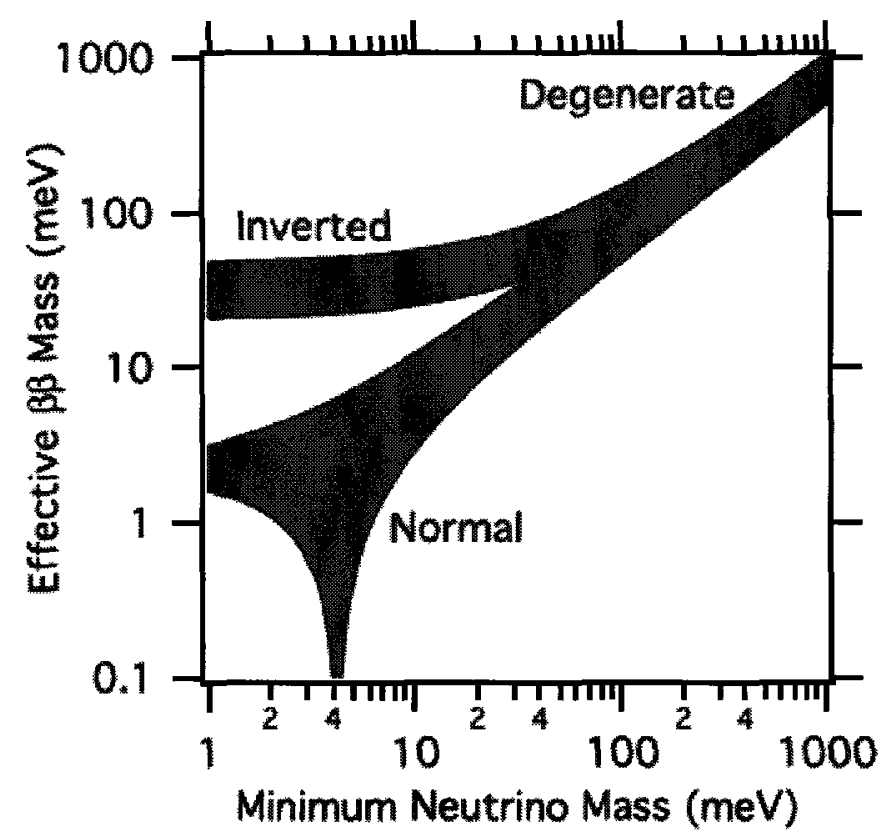

FiguRE 1.5: Double beta decay mass as a function of the minimum neutrino mass. Reprinted with permission from [2], API Copyright 2008. 


\subsection{Overview}

The following chapters will describe my research and contribution towards the development of a high pressure xenon based detector proposed by the EXO collaboration. One of the key requirements is an exceptional energy resolution, on the order of $1 \%$, in order to be able to differentiate the rare neutrinoless peak from the large two neutrino peak and any backgrounds present in the sum electron energy spectrum (see Figure 1.4. Xenon gas will be used as the active detector medium and the main focus of the reseach was to investigate xenon's light production properties and the attainable ionization signal resolution measured indirectly through the production and detection of electroluminescence (EL) light as a function of applied drift field.

The project consisted of optimizing a natural xenon detector located at Carleton for energy resolution measurements via EL light, and investigating the use of EL as a method for ionization signal amplification. Chapter 2 discusses the experimental requirements and the proposed EXO detector along with the required background theory on ionization as well as scintillation and EL light production in

xenon gas. Chapter 3 describes the detector in detail and the specifics of EL generation within it, along with the calibration of the photomultiplier tubes. Chapter 3 concludes with a description of the data acquisition setup and a look at the raw data obtained from the detector. Chapter 4 describes Monte Carlo simulations 
performed to determine the detector efficiency and check drift times. Chapter 5 presents the data analysis and results. The thesis concludes with Chapter 6 and a discussion of the results. 


\section{Chapter 2}

\section{EXO: Experiment Background}

Invented in 1974 by David Nygren [19], the TPC has become one of the standard devices in particle tracking and identification for use in particle physics [19]. The basic idea was to fill a large volume with a gas and then by introducing constant electric and magnetic fields to the volume, any electrons or ions produced in the volume due to ionizing particles will drift along the electric field lines to readout planes located at the ends of the volume. An axial magnetic field is necessary to measure the momentum of the particles, and can help in reducing the diffusion of the drifting charge distributions. Electrons and/or ions drift towards the readout plane where a two dimensional coordinate is recorded with respect to the plane. Drift time measurements of the electrons to the the anode grid provide the third coordinate, z. For electrons or ions drifting in a constant electric field the drift 
velocity for a particular gas or gas mixture is constant due to collisional processes in the gas, with typical velocities being on the order of one to a few $\mathrm{cm} / \mu s$ [19].

The original concept for acquiring the signal produced by the drifting electrons was to incorporate the existing technology of the multi-wire proportional chamber (MWPC) by having a single plane MWPC on either end of the TPC [19]. The design is based on direct charge measurements and due to the often feeble signals charge amplification is often required. The charge gain is generally implemented by applying a sufficiently high electric field which gives the electrons enough energy to participate in ionizing collisions during drift. Multi-wire gaseous detectors have progressed considerably over the decades since their invention, first to micro-strip gas detectors and further to micro-patterned gas detectors (MPGD) [20], micro-mesh gaseous detectors (Micromegas) [21], and gas electron multipliers (GEM's) [22], all of which which offer increased charge amplification, high precision, and can accommodate high event rates. The often large volume and drift distances employed in TPC's give large $d E / d x$ losses which can be used for particle identification, where $d E / d x$ is the ionization energy lost by a charged particle in traversing a unit distance in a material. Particles with different velocities will result in different $d E / d x$ losses allowing for particle identification by mass in a momentum analysis. The TPC is a fully 3-D tracking device which can support high event rates even in a high background environment [19]. 
EXO is a proposed next generation ton scale experiment. EXO will search for the neutrinoless double beta decay of ${ }^{136} \mathrm{Xe}$ by developing a tracking timeprojection chamber (TPC) which utilizes ${ }^{136} \mathrm{Xe}$ as both the source and detector medium. EXO desires to measure the neutrino mass scale through the study of neutrinoless double beta decay and to make precise measurements of the decay rate for both $\beta \beta_{2 \nu}$ and $\beta \beta_{0 \nu}$ in ${ }^{136} \mathrm{Xe}$. EXO is an international collaboration with two avenues being pursued in detector prototyping towards the final design of a ton scale experiment. Collaborating partners in the United States are leading the first initiative with the development of a $200 \mathrm{~kg}$ liquid xenon (LXe) TPC (EXO 200) located in the Waste Isolation Pilot Plant (WIPP) in Carlsbad New Mexico. The detector is fully constructed and undergoing system tests and is expected to begin taking data by the end of 2010 , with preliminary results being expected in the next year. Canada leads the efforts in the second avenue of pursuit, which is the development of a high pressure gas phase Xe TPC. This prototype currently under construction at Carleton University. The choice of using a TPC design for the detector has the main advantage of keeping background producing materials (i.e. everything but the xenon) away from the centre of the detector. The use of a TPC also allows for the reconstruction of ionization tracks. Ideally the number of tracks in each event would be determined and the energy loss per unit pathlength $(d E / d x)$ of each track measured to identify electron like tracks. Events with two electron like tracks emitted from the same vertex, and exhibiting the 
correct energy spectrum, would provide a strong experimental signature for the decay while single track events or multiple track events at disjoint locations could be rejected.

The general properties of xenon gas are listed in Table 2.1. Xenon has over 49 isotopes, where two are predicted to undergo double beta decay ${ }^{134} \mathrm{Xe}$ and ${ }^{136} \mathrm{Xe}$. Until confirmation by measurement of the double beta decay of ${ }^{134} \mathrm{Xe}$ and ${ }^{136} \mathrm{Xe}$ the two isotopes are considered stable, giving a total of 9 stable xenon isotopes and over 40 unstable ones [23]. The choice to use ${ }^{136} \mathrm{Xe}$ for the double beta decay isotope offers many fundamental advantages over ${ }^{134} \mathrm{Xe}$ and other radioactive isotopes. Xenon can be enriched by centrifuging, has a relatively high natural abundance $(\sim 8.9 \%)$, and is fairly easy to purify, therefore obtaining the large amounts required (on the order of 1 ton) is possible. Also, xenon has no other isotopes with long lifetimes and has a relatively high Q-value for the decay $\left(Q_{\beta \beta}=2.48 \mathrm{MeV}\right)$ therefore lightening the background suppression requirements [24]. The expected full width at half maximum (FWHM) intrinsic energy resolution is $\delta E / E=2.7 \times 10^{-3} F W H M$ at the $Q_{\beta \beta}$ value [24]. Another enticing property of xenon is that it can be implemented as both the active mass and detecting medium due to being an efficient scintillator. The scintillation properties also allow for a design based on accurate, well understood optical detection technologies. Another unique and very promising feature of ${ }^{136} \mathrm{Xe}$ is the possibility to use laser spectroscopy to positively identify the daughter nucleus of the decay, 


\begin{tabular}{|c|c|}
\hline \multicolumn{2}{|c|}{ Xenon Gas } \\
\hline Symbol & Xe \\
Description & Noble Gas \\
Atomic Number & 54 \\
Standard atomic weight & $131.293(6) \mathrm{g} \mathrm{mol}^{-1}$ \\
density @ 1 atm \& 293 K & $0.00588 \mathrm{~g} \mathrm{~cm}^{-3}$ \\
Melting point & $161.3 \mathrm{~K}$ \\
Boiling point & $165 \mathrm{~K}$ \\
Electron configuration & {$[\mathrm{Kr}] 5 \mathrm{~s}^{2} 4 \mathrm{~d}^{10} 5 \mathrm{p}^{6}$} \\
\hline
\end{tabular}

TABLE 2.1: Xenon properties table.

${ }^{136} \mathrm{Ba}^{++}$, thereby eliminating all backgrounds that do not produce the required ion. All of the aforementioned points make ${ }^{136} \mathrm{Xe}$ very relevant in the search for neutrinoless double decay. The experimental requirements for the $\beta \beta_{0 \nu}$ search will now be addressed along with how xenon gas can be used to meet the requirements.

\subsection{Ionization \& Scintillation in Xenon}

This section will describe the production of scintillation light due to the passage of an ionizing particle in xenon gas. When an ionizing particle passes through a gas it loses its energy though elastic and inelastic collisions and radiative loss such as bremsstrahlung. Elastic collisions only become the dominant energy loss mechanism for heavy particles at the end of their track, and bremsstrahlung losses only become important if the kinetic energy of the particle is much greater then its rest energy. Inelastic collisions result in the excitation and ionization of the 
atoms and molecules of the gas. The main energy loss mechanism in the gas is due to kinetic energy imparted to the primary ionization electrons. The primary ionization electrons also go on to excite and ionize the gas until they reach subexcitation energies at which point they lose their remaining energy through elastic collisions or inelastic collisions that do not involve electronic excitations. In the absence of an electric field the electrons, once thermalized, recombine with the ions in the gas or with an existing boundary [25].

The energy pathways available for a charged particle which deposits all of its kinetic energy in a gas are represented by the energy balance equation of Platzman $[26,27]$

$$
E_{0}=N_{i} E_{i}+N_{e x} E_{e x}+N_{i} \epsilon_{h}
$$

where $E_{0}$ is the kinetic energy of the charged particle, $N_{i}$ represents the average number of electrons produced by the incident particle, $E_{i}$ the average ionization potential required to liberate an electron from the gas atoms, $N_{e x}$ the average number of excited atoms, $E_{\text {ex }}$ the average potential to excite an atom of the gas, and $\epsilon_{h}$ the average energy of subexcitation electrons. The average energy required to liberate a single electron is given as the $\mathrm{W}$-value for ionization, which is defined as: 


$$
W_{i o n}=\frac{E_{0}}{N_{i}} .
$$

The expression for the average energy required to excite a xenon atom, represented by the $\mathrm{W}$-value for excitation is:

$$
W_{e x}=\frac{E_{0}}{N_{e x}} .
$$

When considering scintillation yield, an analogous $\mathrm{W}$-value is defined: the average energy required to produce a single scintillation photon:

$$
W_{s}=\frac{E_{0}}{N_{s}} .
$$

where $N_{s}$ is the average number of scintillation photons produced by the incident particle. While the value of $W_{i}$ is roughly the same for all types of incident particles the value for $W_{s}$ is highly dependent on the particular particle, mostly due to the different ionization densities that result from each particle. While in the absence of recombination $W_{s}$ is equivalent to $W_{e x}$, in general they are not equal because there are two distinct origins of scintillation light in xenon [27].

The production of scintillation light in pure xenon is the end result of a complex set of elementary processes which can be broken down into three main stages [25]: 
- The primary particle and all the resulting ionization electrons are slowed down creating primary activation states with large energies. This part of the process occurs very quickly, $\sim 10^{-9} \mathrm{~s}$ for a typical gas at atmospheric pressure.

- These primary states of excitation and ionization either become radiative states or de-excite by other energy loss mechanisms.

- The radiative states decay back to the ground state with the emission of a vacuum ultraviolet photon.

After the first stage the gas contains electrons, neutral atoms, and atoms excited in several levels, as well as excited and non-excited ions which are mostly singly charged. For very low pressure gas, the majority of excited atoms deexcite radiatively due to a lack of atomic collisions. The resulting spectrum is essentially just atomic. For a gas under pressure the excited atoms participate in binary and ternary processes and form other excited molecular species that decay radiatively and contribute to the observed spectrum [25]. These processes result in a characteristic xenon spectrum in the vacuum ultra-violet (VUV) which exhibits two Gaussian emission continua, peaked at $147 \mathrm{~nm}$ and $178 \mathrm{~nm}$, named the first and second continuum respectively [28].

The first continuum is peaked very near to the resonance line and its emission is thought to be due to transitions from xenons vibrationally unrelaxed and 
excited $0_{\mu}^{+}\left({ }^{1} \sum_{\mu}^{+}\right)$molecular states to the repulsive ground state [29]. The second continuum is believed to originate from the radiative decay of lowest lying, vibrationally relaxed excited molecular states, $(\nu=0){ }^{1} \sum_{\mu}^{+}$and ${ }^{3} \sum_{\mu}^{+}$, to their repulsive ground states[29]. Xenon forms attractive excited diatomic molecules (excimers) through the interaction of excited atoms, ions and subexcitation electrons with the neutral atoms of the gas. The first continuum is only present at low pressures, and at pressures above a few hundred Torr the second continuum completely dominates the spectrum $[28,30]$. The second continuum is of greater interest since all planned detectors will be operated at or above atmospheric pressure. For a gas above a few hundred Torr [26], the basic reactions responsible for the formation and decay of xenon excimers with the emission of scintillation light can be represented by the two processes:

1.

$$
\begin{gathered}
X e^{+}+2 X e \rightarrow X e_{2}^{+}+X e, \\
e_{h o t} \rightarrow e_{t h}+h e a t\left(=\epsilon_{h}\right) \\
X e_{2}^{+}+X e+e_{t h} \rightarrow X e^{* *}+X e, \\
X e^{* *}+X e \rightarrow X e^{*}+X e, \\
X e^{*}+X e+X e \rightarrow X e_{2}^{*}+X e,
\end{gathered}
$$




$$
X e_{2}^{*} \rightarrow 2 X e+h v .
$$

2.

$$
\begin{gathered}
X e^{*}+X e+X e \rightarrow X e_{2}^{*}+X e, \\
X e_{2}^{*} \rightarrow 2 X e+h v .
\end{gathered}
$$

Here $X e^{+}$denotes an atomic ion, $e_{t h}$ a thermalized electron, $e_{h o t}$ a electron not in thermal equilibrium with the gas, $X e^{*}$ a singly excited atom, $X e^{* *}$ a doubly excited atom, $X e_{2}^{+}$a molecular ion, $X e_{2}^{*}$ an excited molecular state, and $\mathrm{E}_{\gamma}=\mathrm{hv}$. Scintillation photons originating from the first set of processes are referred to as recombination luminescence while those which originate from the second set are refereed to as excitation luminescence. The wavelength of the scintillation light emitted from either process is $178 \mathrm{~nm}$ with a FWHM of about $15 \mathrm{~nm}$ [27].

A major difference between the scintillation light produced from excitation and recombination luminescence is that recombination luminescence is dependent on the applied electric field. Process (2.7) relies on the Coulomb attraction between the molecular ion and the thermalized electron. If an electric field with a high enough strength to overcome the Coulomb force is applied, recombination is prevented, at which point $W_{s}=W_{e x}$ [27]. For heavy ionizing particles such as $\alpha$-particles, two structures with different ionization densities are thought to form around the primary particle's trajectory. The region of high ionization density 
formed along, and very close to, the primary particle's path due to collisions with the xenon atoms is called the core. The region of moderate ionization density called the penumbra is formed by secondary ionization electrons and surrounds the core region [26]. Recombination in the core (geminate recombination) is very fast and occurs with a near $100 \%$ probability unless a strong electric field is applied. Recombination in the penumbra (volume recombination) is much slower, where even with no electric field, some electrons can escape recombination completely due to becoming thermalized away from their parent ions [27]. All this leads to the formation of excimers in the lowest lying vibrationally relaxed excited states, each state characterized by its spin orientation, labelled singlet and triplet states and denoted by ${ }^{1} \sum_{\mu}^{+}$and ${ }^{3} \sum_{\mu}^{+}$. The most notable difference between the singlet and triplet states is the time for radiative decay to the repulsive ground state, with the singlet and triplet states having time constants of $\tau \sim 5 \mathrm{~ns}$ and $\tau \sim 100$ ns respectively [25]. The decay of these excimers to their ground states $\left({ }^{1} \sum_{\mu}^{+} \rightarrow{ }^{1} \sum_{g}^{+}\right)$and $\left.{ }^{3} \sum_{\mu}^{+} \rightarrow{ }^{3} \sum_{g}^{+}\right)$is the basis for the observed scintillation spectrum for pure xenon gas due to the passage and/or absorption of ionizing particles.

The present work is concerned with scintillation and EL light production from alpha particles in xenon gas, and representative $\mathrm{W}$-values obtained from different authors are presented in Table 2.2. For the particular measurement methods 


\begin{tabular}{|c|c|c|c|}
\hline \multicolumn{5}{|c|}{ W-values for alpha particles in Xe } \\
\hline Reference & Density/Pressure & $W_{\imath}(\mathrm{eV})$ & $W_{s}(\mathrm{eV})$ \\
\hline$[31]$ & $1.01 \times 10^{5}-1.01 \times 10^{6} \mathrm{~Pa}$ & $21.0 \pm 0.4$ & $34.3 \pm 1.6$ \\
{$[32]$} & $1.01 \times 10^{5} \mathrm{~Pa}$ & $21.9 \pm 0.4$ & $49.6 \pm 1.1$ \\
\hline
\end{tabular}

TABLE 2.2: Literature $\mathrm{W}$-values.

and a comparison to values obtained from other work the interested reader is directed to the citations in the table and references therein. The values for $W_{\imath}$ are consistent throughout the literature, whereas $W_{s}$ values have been reported that range from (34.3-49.6) eV [27, 31]. For analysis purposes particular $\mathrm{W}$-values had to be chosen, and the range in the $\mathrm{W}$-values presented introduces an uncertainty in some of the results. These issues will be addressed in Chapter 5 .

\subsection{Electroluminescence in Xenon}

Electroluminescence (EL) in noble gases has been used in particle detectors since the 1970's, and mainly used in devices called Gas Proportional Scintillation Counters (GPSC) for high-resolution spectroscopy of low energy X-rays [24]. EL in xenon, and similarly for all the noble gases, is the emission of secondary scintillation photons due to the drift of ionization electrons under the influence of sufficiently high electric field. The production of EL is the result of scattering processes between the drifting electrons and the surrounding gas atoms and therefore highly dependent on the elastic and inelastic scattering cross-sections. Scattering 
cross-sections are energy dependent, with the amount of energy that each electron receives being directly proportional to the electric field strength, with the probability of interaction increasing relative to the gas number density/pressure. EL yield is therefore commonly given as a function of the reduced electric field, either in terms of $\mathrm{N}$, the number density of the gas $\frac{E}{N}$, or p, the gas pressure $\frac{E}{p}$, where $\mathrm{E}$ is the electric field. Results from spectral analysis and efficiency measurements indicate that the production of EL photons below the threshold for gas gain $\left(\frac{E}{p}<8-9 V /\right.$ cmTorr, $\left.[24]\right)$ is the result of decaying excimers [33, 34], displaying only the characteristics of the second continuum [34]. The production of EL has a very good scintillation efficiency, defined as the fraction of energy given to the drifting electrons by the field which is used for excitation [33].

In the production of EL an electron drifts from regions of low field to high field regions under the influence of a sufficiently strong electric field. As the electron drifts it undergoes inelastic collisions, all the while gaining energy from the field. If the field is strong enough the electron continues to gain energy until enough is gained for the electron to participate in inelastic collisions with the neutral xenon atoms. If the field is such that the drifting electron does not gain enough energy to ionize the gas, the inelastic collisions result only in atomic excitations $\left(X e+e^{-} \rightarrow X e^{*}+e^{-}\right)$. The excited xenon atoms go on to participate in reaction (2.11) of Section 2.1 forming excimers. The excimers subsequently decay to the ground state producing scintillation photons. 
In determining the expected photon yield for a given field and gas pressure, the parameter of interest is the number of inelastic collisions occurring for a single electron, per unit length of drift[34]. Results for the EL photon yield obtained from Boltzmann calculations and Monte Carlo simulations for the generation of EL light, assuming a 1-1 correspondence for emitted photons to atomic excitations, are in good agreement with the data [33-36]. Assuming this model of EL photon generation it is expected that the EL yield should have a linear dependence on the length of the EL gap, the pressure, and the electric field for $E_{0}<\frac{E}{p}<T_{g}$, where $\mathrm{E}_{0}$ is the threshold for EL production and $\mathrm{T}_{g}$ is the threshold for gas gain. This assumption is supported by a number of authors from the analysis of various data sets $[3,37,38]$.

For a constant, uniform $\frac{E}{p}$ with a total voltage of $\Delta V$ the total EL gain is defined as the number of photons generated per electron per unit of drift distance, and can be represented as [24],

$$
N_{E L p h}=\frac{\Delta V}{V_{E L p h}}
$$

where $\mathrm{V}_{E L p h}$ is the average potential required to produce one EL photon $\left(\mathrm{V}_{E L p h}=10\right.$ ev), for a set $\frac{E}{p}$. An empirical formula obtained for xenon gas giving the total EL gain, $\mathrm{N}_{E L p h}$, as a function of $\frac{E}{p}$ is given by [3], 


$$
d N_{E L p h}=A\left[\frac{E}{p}-B\right] p d x \quad\left(V U V \frac{\text { photons }}{e \quad c m}\right)
$$

where $\mathrm{A}$ is an amplification parameter with units of photons $/ \mathrm{kV}, \mathrm{E} / \mathrm{p}$ is the reduced electric field with units of $(\mathrm{kV} / \mathrm{cm}-\mathrm{bar}), \mathrm{B}$ is a threshold parameter for EL generation with the same units as $\mathrm{E} / \mathrm{p}, \mathrm{p}$ is the pressure in bar, and $\mathrm{dx}$ is the drift distance in the EL region in $\mathrm{cm}$. Recent results of Monteiro et al. [3] give values of $\mathrm{A}=140$ (photons $/ \mathrm{kV}$ ) and $\mathrm{B}=0.83(\mathrm{kV} / \mathrm{cm}$-bar) in good agreement with Boltzmann and MC studies [3]. Earlier papers reported values for the EL amplification and threshold parameters as being $\mathrm{A}=70$ (photons $/ \mathrm{kV})$ and $\mathrm{B}=1.0(\mathrm{kV} / \mathrm{cm}-\mathrm{bar})$ respectively, however these values were later attributed to gas contamination. A compilation of the results obtained for the amplification and EL threshold parameters under various conditions can be found in [3]. Optical gains up to 1000 photons/e have been generated using EL based detectors [3, 24].

\subsection{Enriched Xenon Observatory (EXO): Gas-}

\section{phase}

The EXO collaboration has begun prototyping a gas phase high pressure xenonbased TPC, designed specifically to search for the neutrinoless double beta decay of ${ }^{136} \mathrm{Xe}$. The finalized concept has also incorporated various important design 
suggestions made by David Nygren. To meet the desired energy resolution and tracking requirements, the detector will rely on exploiting xenon's scintillating properties for signal detection using a cylindrical high-pressure xenon (HPXe) TPC, up to 10 bar, implementing a separated function design concept. The separated function approach is essentially to measure the tracking and energy resolution separately using technology specifically optimized for each purpose. The TPC will be based on the conversion of an ionization signal to a light signal via electroluminescence, which will be detected by two arrays of cesium iodide photo-cathodes. There are two main advantages in using a high-pressure xenon TPC design. One is the choice to use gaseous xenon instead of liquid xenon, which is partly due to their respective Fano factors.

The Fano factor represents the intrinsic fluctuations in the partitioning of deposited energy between ionization and other energy loss mechanisms, where $\mathrm{F}$ is generally less than unity [24]. LXe is has been shown to have an anomalously large Fano factor, $\mathrm{F}_{L X e} \sim 20$ which makes reaching the goal of optimal energy resolution difficult [24]. Gaseous xenon however, has a Fano factor of $\mathrm{F}_{G X e}=(0.15-0.20)$ [24], giving it an excellent intrinsic energy resolution. The large difference in the two Fano factors implies that energy resolution in LXe is worse than in gaseous xenon by a factor of $\frac{\sigma(E)_{L X e}}{E} / \frac{\sigma(E)_{G X e}}{E} \sim 11.5$. This alone implies that using a HPXe TPC is a worthwhile approach towards optimizing detector energy resolution for 
the $\beta \beta_{0 \nu}$ search, though both approaches are feasible when translated to an inherent resolution at $2.48 \mathrm{MeV}$. By measuring the scintillation signal and exploiting the anti-correlation between ionization and scintillation, EXO-200 improves the energy resolution in LXe. However, xenon's intrinsic resolution cannot be fully restored to its expected value since precise measurement of the scintillation signal for a particular event is not possible [24].

\subsubsection{XEP: A High Pressure Xe (HPXe) Electrolumines- cent TPC}

The Carleton portion of EXO is leading the way in developing a high-pressure xenon gas phase prototype (XEP), which will be built and operated at Carleton. The basic detector concept for XEP is illustrated in Figure $2.1 \mathrm{a}, \mathrm{b}$, and c. Initially, there is an ionization event which occurs in the active volume of the detector followed by a prompt burst of scintillation light. Next, the resulting ionization electrons are transported through the gas by means of a constant electric field to the high field EL region. While traversing the EL region each electron produces an amount of EL light proportional to the distance travelled, and the electric field strength in the region. The drift field will run along the axis of the cylindrical chamber with the EL region being at one end of the chamber. The prototype has a inner radius of $60 \mathrm{~cm}$ and a length of $123 \mathrm{~cm}$ and 28 field shaping rings. 


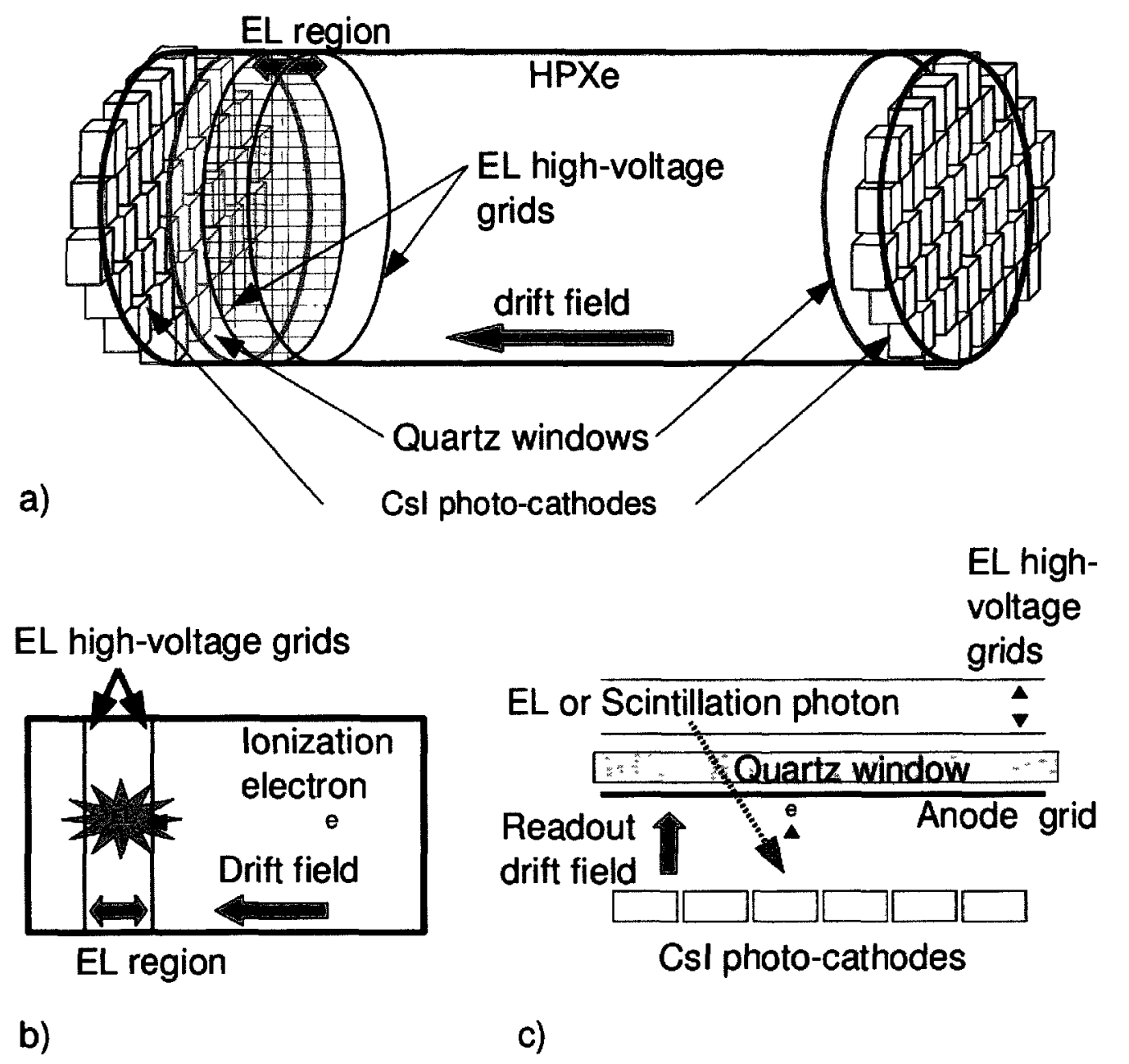

FIGURE 2.1: XEP design concept.

Each ring will be made from copper and oriented with its enclosed area normal to the $\mathrm{E}$ field along the drift path. A voltage divider chain will supply voltage to the cathode and rings producing a uniform $\mathrm{E}$ field in the drift region. The light must be transmitted out of the xenon and into a methane environment of the readout pads. The methane allows for additional signal gain through gas amplification if desired. To accomplish this, two quartz windows will be used 
which have excellent transmission for xenon scintillation light $(\approx 178 \mathrm{~nm})$. To generate the high-field EL region, a voltage difference will be applied to two large wire or mesh grids positioned approximately $1 \mathrm{~cm}$ apart. The anode mesh for collecting the drifting electrons will either be deposited directly onto the quartz window or positioned directly in front of it. The primary scintillation light and the later arriving EL light are then collected by readout packages on both ends of the TPC, while the drifting electrons are collected by an anode mesh. Each readout package will consist of approximately $200 \mathrm{CsI}$ readout pads which will allow for accurate tracking and energy resolution measurements to be made. As each event occurs and the ionization electrons drift through the EL region, the light is recorded by the readout packages on either side of the detector. The signals induced on the readout pads on the end closest to the EL region are then used for tracking and position reconstruction, while the signals recorded on the end furthest from the EL region are used for energy resolution. The signal recorded on the readout plane on the near side to the EL region will be nonuniform over the readout pads and highly dependant on the radial position of the light generation, making it ideal for tracking purposes. The signal recorded on the readout plane on the far side to the EL region will be uniform over the readout pads with a soft cosine-like dependence with the radial position of the light generation, making it well suited for energy measurements. This is the main concept of a separated function TPC first proposed by David Nygren [24]. The principle of detection of 
a single photon by a CsI readout pad is illustrated in Figure 2.1c. Cesium iodide is a photo-sensitive substance that has a work function which is lower than the energy carried by the scintillation photons. As a photon hits the pad an electron is knocked off the surface of the CsI coating by the photoelectric effect. There will be an anode mesh on the quartz window and an applied electric field between it and the CsI photo-cathodes. The ejected electron will drift through the methane inducing a current on the readout pad, until being collected by the anode mesh. The readout system can be operated with variable gas gain as desired.

The main advantage of choosing the separated function design along with EL generation is that no event boundary is required for an energy measurement as long as the reconstructed tracks lie within the fiducial volume of the detector including diffusion effects. This is because all electrons contribute equal statistical weight to the total EL signal [24]. There are a number of advantages in using CsI photo-cathodes to collect the light, most notably: CsI photo-cathodes have been shown to have a quantum efficiency (QE) on the order of (30-40)\% for converting a UV optical signal into an electrical signal, and being much more cost effective then photo-multiplier technology allows for a highly pixelated readout design to gather tracking information for position reconstruction. These are the key aspects of the approach which allow for the possibility of achieving an optimal energy and positional resolution for making an accurate measurement of the neutrinoless double beta decay of ${ }^{136} \mathrm{Xe}$. 


\subsection{Experimental Requirements}

Neutrinoless double beta decay is an extremely rare process and the experimental search for a positive signature of the decay is challenging. In designing and optimizing a detector for the $\beta \beta_{0 \nu}$ search, there are a number of key considerations. The three main considerations are as follows:

- energy resolution,

- track reconstruction and positional resolution,

- background suppression and rejection.

\subsubsection{Energy Resolution for $\beta \beta_{0 \nu}$}

The main requirement for a large scale xenon $\beta \beta_{0 \nu}$ experiment to be successful and achieve a sensitivity on the order of $50 \mathrm{meV}$ an energy resolution of $1 \%$ or better. The $m_{\beta \beta}$ sensitivity is dependent on the background, the ${ }^{136} \mathrm{Xe}$ mass, and the experiments lifetime. In order to accurately identify a $\beta \beta_{0 \nu}$ event the detector must be able to resolve the highly suppressed $0-\nu$ peak from the large $2-\nu$ distribution. The $2-\nu$ peak (see Figure 1.4) is an irreducible background which can only be distinguished by energy and therefore the better the energy resolution the fewer $2-\nu$ events in the energy search window. The background is limited in 
the end (i.e. perfect non $\beta \beta$ event rejection) by how many $2-\nu$ events end up in the window and hence by the energy resolution. The intrinsic resolution is defined as

$$
\frac{\delta E}{E}=2.35 \sqrt{\frac{F W_{\imath}}{E}} \quad(F W H M),
$$

where $\mathrm{E}$ is the deposited energy, $\mathrm{F}$ is the Fano factor, and $W_{\imath}$ is the average energy needed to produce an electron/ion pair.

For xenon gas, the ionization Fano factor has been found to be $\mathrm{F}=0.15 \pm 0.02$, with $W_{\imath}=21.9 \pm 0.4 \mathrm{eV}$ [24], and taking $\mathrm{E}=\mathrm{Q}_{0 \nu \beta \beta}=2.48 \mathrm{MeV}$, the intrinsic resolution is $\delta E / E=2.7 \times 10^{-3} F W H M$ as stated previously. Currently there is no estimation of the Fano factor for scintillation $F_{s}$, however, in assuming that each recombined electron-ion pair produces one scintillation photon it is predicted that $F_{s} \leq F[39]$.

In theory the only factor that affects the attainable energy resolution is the Fano factor. In practise however there are a number of factors which determine the resolution. The energy is found by counting the number of ionization electrons. In order to overcome various limitations such as electronic noise at signal processing and detector efficiency the signal is expected to be measured by a method that introduces gain. Whichever gain technique is used introduces another Fano-like 
factor which can degrade the energy resolution, represented by G. Assuming G is uncorrelated with $\mathrm{F}$, the equation for the energy resolution becomes.

$$
\frac{\delta E}{E}=2.35 \sqrt{\frac{(F+G) W_{\imath}}{E}} \quad(F W H M)
$$

To achieve optimal energy resolution under these circumstances would require $\mathrm{G} \leq \mathrm{F}[24]$, and therefore it is desirable to design a detector which minimizes $\mathrm{G}$ as much as possible. Assuming $\mathrm{F}=0.15$, achieving gain factors this low for charge amplification methods has yet to be shown possible [40]. It has been proposed that gain factors comparable to this can be achieved by using signal amplification via optical gain through the generation of electroluminescence.

The use of EL gain allows for the minimization of effects that impact the energy resolution post EL light generation such as photo-cathode QE, light loss from attenuation, and solid angle coverage. Generally factors like these are constant value contributors to fluctuations in the obtained EL signal $\left(\sigma_{E L}\right)$ or functions of $N_{E L}$ such as $1 / \sqrt{N_{E L}}$, therefore signal gain reduces their overall contribution. To achieve the optimal resolution the condition that must be met for any gain process employed is that the variance in the gain must be on the order of the Fano factor $[24,41]$, otherwise any benefits of the small Fano factor for gaseous xenon would be lost. The choice of signal amplification and detection via EL optical gain offers a very good conversion efficiency with a low statistical variance. Each electron 
travels through the same potential, producing scintillation photons sequentially in the absence of charge gain. This gives rise to normal fluctuations in the number of scintillations [24]. The $\mathrm{G}$ variance in Equation (2.16) must include contributions from fluctuations in the optical gain $\left(N_{E L}\right)$, the number of detected EL photons per electron $\left(N_{\text {detected }}\right)$, and the gain process of the photodetector $\left(\sigma_{p d}\right)$ [24]. To a good approximation $\mathrm{G}$ can be given as [24],

$$
G=1 / N_{E L}+\left(1+\sigma_{p d}^{2}\right) / N_{\text {detected }}
$$

The first term is much smaller than the second, and can generally be neglected since $N_{E L}<<N_{\text {detected }}$ due to limited light collection efficiency, typically 1-3\%. As an example consider the following case which employs signal detection with PMTs. Modern PMTs have $\sigma_{p d}^{2}<0.5$ [24]. Here a value of 0.5 will be assumed. To obtain $G \leq F \approx 0.2$ in Equation (2.17) the number of detected EL photons must satisify $N_{\text {detected }}>8$. The number of detected EL photons is $N_{\text {detected }}=($ light collection efficiency $) \times N_{E L}$. Assuming a $2 \%$ light collection efficiency results in $N_{E L}>400$ (photons/electron). While this gain value is large, it is well within experimentally measured optical gain values [3], implying that it is reasonable to achieve a $\mathrm{G}$ variance on the same order as the Fano factor. If the number of detected photons per electron is large enough, the associated fluctuations can have much less of an effect on the resolution then the intrinsic 
fluctuations represented by the Fano factor.

The generation and subsequent detection of large numbers of photons seems reasonable for signal amplification by EL since very high optical gains can be reached with moderate electric fields. The generation of EL has a number of intrinsic characteristics which make it attractive for signal extraction: the sensitivity of EL gain to density and field is small compared to charge avalanche devices, the possibility of single electron sensitivity with a good signal to noise ratio, and minimal space charge limitations due to the absence of positive ion production in the detection process [24]. These points along with the normal fluctuations of the EL gain process give the possibility to achieve a energy resolution unsurpassed by other gas amplification processes [24].

The high-gain, low-noise, low-fluctuation conversion of an ionization signal to a light signal via electroluminescence has a very low statistical variance, presently better than any other gain process, and also allows for easy and flexible signal detection using the large assortment of known optical detection techniques [24]. An estimate for the energy resolution attainable at the $\beta \beta_{0 \nu}$ Q-value using optical gain from EL, is given as $\delta Q / Q=4.2 \times 10^{-3} F W H M$ [24]. This estimate is supported by the work of Bolozdynya et al. [42], where they obtained $\delta Q / Q=4.4 \times 10^{-3} F W H M$ using a HPXe EL drift chamber and extrapolating their result for $122 \mathrm{keV}$ X-rays to the Q-value as $E^{-\frac{1}{2}}$ [24]. The detection of the 
prompt scintillation light also offers a simple method for obtaining a event start time for the TPC, though this requires the signal collection to be optimized for the large EL signals while also being sensitive to the much smaller scintillation signal.

Other factors which have an impact on the energy resolution are: ionization losses due to electron capture by internal structures of the detector or by electronegative impurities, bremsstrahlung losses, fluctuations in the specific ionization yield due to the exact conditions of field and pressure, along with factors such as readout response stability and nonlinearities. For a more detailed discussion of the factors specifically pertaining to the $\beta \beta_{0 \nu}$ search, the reader is refereed to $[24,40]$. The specific factors effecting energy resolution which pertain to the present work will be addressed later.

\subsubsection{Tracking for $\beta \beta_{0 \nu}$}

In order to identify a true $\beta \beta_{0 \nu}$ event with a high level of certainty, a considerable effort must be made to be able to distinguish these true events from background. For a particular event it may also be necessary to determine precisely which peripheral readout channels contribute to the actual signal and which contribute to noise in order to obtain the most accurate energy measurement [40]. The ability to 
accurately reconstruct the ionization tracks created by the decay electrons can provide a powerful method for gathering this information. The two electrons emitted in the decay have specific geometric topologies, making identification somewhat straightforward. Multiple scattering in xenon is large and electrons at the end of their trajectories will lose any remaining energy to elastic scattering, forming Bragg peaks in the $d E / d x$ distribution for the event, giving the endpoints of reconstructed tracks obvious "blobs" characteristic of Bragg scattering. These blobs make the task of tracking more difficult however due to the convolution of overlapping trajectories. Even with very good position resolution it is not expected that the individual trajectories making up the blobs could be resolved, and the region of the blobs will not be accurately reconstructed. Detailed track reconstruction for the region corresponding to the energy peak is not essential as long as the presence of the blobs can be recognized. Track reconstruction will also determine whether or not an event has occurred completely within the detector's fiducial volume thereby improving energy measurements. The two electron tracks from the decay will have a common vertex, the location of the decay, giving another topological signature. Obtaining these two pieces of information will give powerful topological means to positively identify a true double beta decay event while rejecting background events and determining whether or not a candidate event was fully contained within the fiducial volume. 


\subsubsection{Background suppression and rejection for $\beta \beta_{0 \nu}$}

Positive identification of a $\beta \beta_{0 \nu}$ event requires a very sensitive measurement and in order to reach desired precision and accuracy a detector must have an overall low background rate and the ability to distinguish background events from true $\beta \beta_{0 \nu}$ events. Great care is taken in choosing low background materials for the construction of the detector and advanced methods for event identification are employed. The expected energy released by the decay is $\mathrm{Q}_{\beta \beta}=2.48 \mathrm{MeV}$ and any process which deposits energy in this range must be addressed. The backgrounds which are the most troublesome are due to neutrons and $\gamma$-rays originating outside the detector or within the detector materials, with smaller scale detectors being intrinsically more sensitive to these backgrounds due to a lack of self-shielding. Problematic backgrounds such as decay of ${ }^{214} \mathrm{Bi}$ with the emission of an electron with an energy of $2369 \mathrm{keV}$ would mimic the energy signature of $\beta \beta_{0 \nu}$, and could only be excluded using tracking information [40]. Also, photo-conversion which leads to xenon fluorescence will leave a blob of deposited energy near the original interaction point which could be mistaken for the Bragg peak of an electron track. Therefore recognizing this background with a high efficiency is important [40]. Again it is hoped that accurate track reconstruction will be able to distinguish between these events and true events.

The possibility of identifying (tagging) the daughter nucleus would offer the 
best means of background rejection. The decay of ${ }^{136} \mathrm{Xe}$ results in the daughter nucleus ${ }^{136} B a$, and the positive identification of this daughter would eliminate all backgrounds which did not result in the production of the $\mathrm{Ba}$ ion, providing a very powerful tool for discriminating background events for true events. The most promising method for tagging the $\mathrm{Ba}$ ion is through the use of laser fluorescence. In order to identify the barium ion through laser fluorescence, the ion would have to be transported from the decay site to an ion trap where spectroscopy could be performed. The approach is plausible since $\mathrm{Ba}^{++}$is relatively long lived in $\mathrm{Xe}$ and identification of the barium ion through fluorescence has been demonstrated. In practise however its realization is challenging. 


\section{Chapter 3}

\section{Electroluminescence (EL) Test}

\section{Chamber}

To investigate the optical gain and energy resolution available from an EL xenon detector, a cylindrical EL test chamber was built and operated using natural xenon. The detector was designed to operate at a pressure of 1 bar and obtain an energy resolution of $\sim 1 \%$ using an Americium $241\left({ }^{241} \mathrm{Am}\right)$ alpha particle $(\alpha)$ source of energy $5.49 \mathrm{MeV}$. The chamber has a cylindrical geometry anode/cathode system which produces a cylindrical electric field (E) and EL region. The basic concept is illustrated in Figure 3.2. The formula governing the electric field is given by (3.1). 


$$
E(r)=\frac{V}{\ln (b / a) r}
$$

where $\mathrm{V}$ is voltage in $\mathrm{kV}$, a is the anode radius, $\mathrm{b}$ the cathode radius, and $\mathrm{r}$ the radial position with respect to the axis of the cylinder. Figure 3.1 illustrates the $1 / \mathrm{r}$ dependence of the electric field for various applied voltages and the field strength at which gas gain occurs. Ionization electrons produced in the chamber will drift along the radial field lines experiencing stronger potentials and gaining more energy from the field as they drift towards the anode. At a particular radial distance, $\mathrm{R}_{E L}$, the field strength becomes high enough to satisfy the condition: Equation $(2.14)=0$. Above this field strength each electron gains enough energy to participate in reaction (2.11) of Section 2.1 and produce EL light. An anode radius had to be chosen which did not produce fields that would cause electron multiplication over the range of high-voltages.

In considering a cylindrical drift field, the optical gain formula (2.15) must be modified to account for the nonuniform E/p. Equation (2.14) is a differential equation in the spatial variable $\mathrm{x}$ and was empirically derived for a uniform $\mathrm{E}$. It should however also apply to the case where $\mathrm{E}$ is a function of a spatial coordinate $[24,43]$, in this case the radial position $r$. To obtain the modified form of Equation (2.14), the electric field in Equation (2.14) (E) is replaced with the expression for a cylindrical electric field $\left(\mathrm{E}_{r}\right)$, along with the substitution $\mathrm{dx} \rightarrow \mathrm{dr}$. Integration 


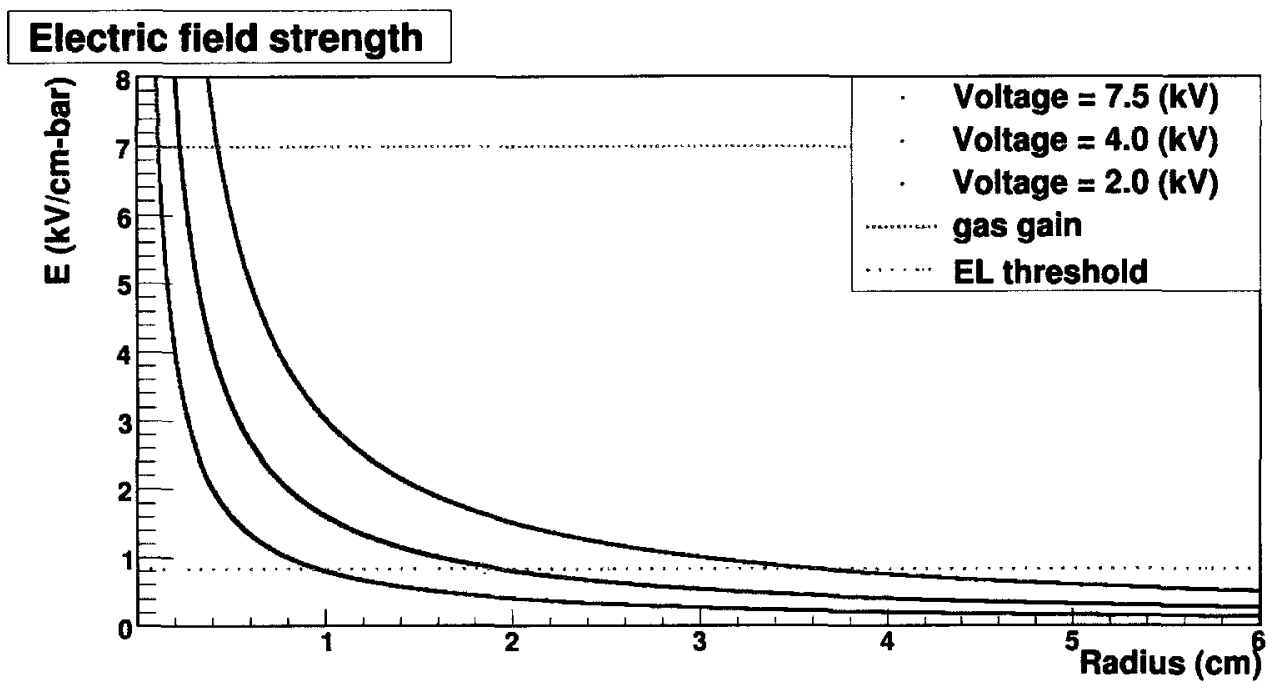

FIgURE 3.1: Electric field vs. radial position.

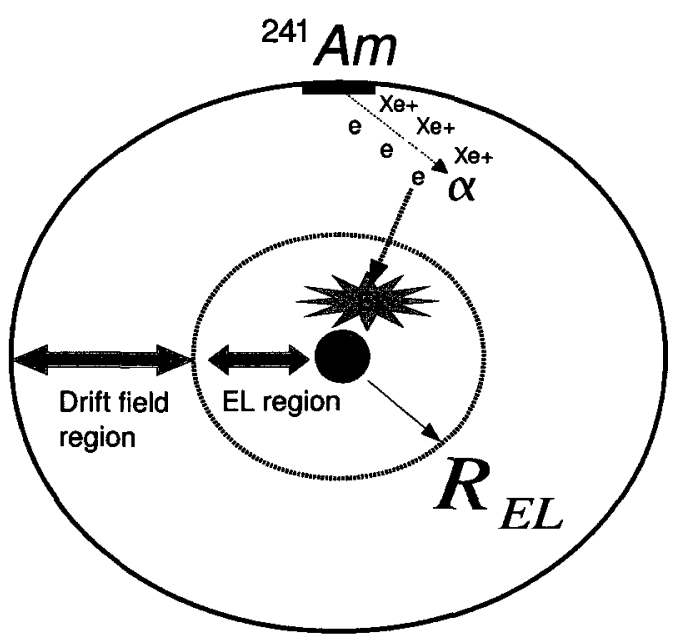

Axial view

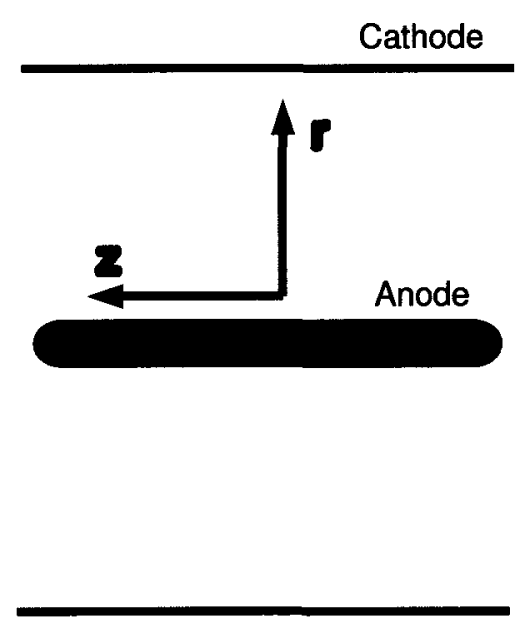

Side view

Figure 3.2: Cylindrical EL detector concept.

is then performed over the radial extent of the EL region $\left(r=a \rightarrow r=R_{E L}\right)$, resulting in the total number of EL photons produced for each electron which 
drifts entirely through the EL region. Using $\mathrm{E}_{r}$ (Equation (3.1) in Equation (2.14) and setting $\mathrm{p}=1$, the expressions obtained for $R_{E L}$ and $N_{E L}$ are:

$$
\begin{gathered}
R_{E L}=\frac{V}{\ln (b / a) B}, \\
\int N_{E L}=\int_{r=a}^{R_{E L}} A[E(r)-B] d r, \\
\int N_{E L}=A\left[\frac{V}{\ln (b / a)}\left(\ln \left(\frac{V}{\ln (b / a) B}\right)-\ln (a)-1\right)+B a\right],
\end{gathered}
$$

where $\mathrm{a}$ is the anode radius, $\mathrm{b}$ is the cathode radius, $\mathrm{A}$ is an amplification parameter and $\mathrm{B}$ is a threshold parameter.

The two main purposes of the detector are to investigate the available EL energy resolution and to investigate EL optical gain as a function of applied voltage. The EL energy resolution is defined here as,

$$
\frac{\delta E}{E}=\frac{\sigma_{E L}}{\text { Mean }_{E L}} .
$$

where $\sigma_{E L}$ and $M e a n_{E L}$ are the Gaussian $\sigma$ and mean obtained from fitting the 
resulting integrated EL distributions. Due to the lack of an absolute calibration of the digitized data, the EL optical gain is determined from using ratio of the integrated signals $\left(M e a n_{E L} / M e a n_{\text {Scint }}\right)$. To obtain the number of EL photons relative to the number of ionization electrons the previous quantity must be corrected using the $\mathrm{W}$-values for ionization and scintillation given in Chapter 2 along with efficiency averaging to account for the light collection efficiency of the detector. The overall light collection efficiency is dependent on the applied voltage due to the changing EL threshold radius, as well as the angular distribution of the ionization tracks. The scintillation region efficiency $\left(S_{\text {efficiency }}\right)$ is defined as the average efficiency over the volume of the detector corresponding to a given angular range of $\alpha$ particle emission. The EL region efficiency $\left(E L_{\text {efficiency }}\right)$ is defined as the average efficiency over the volume of the chamber where EL light is generated corresponding to a given angular range. The relative number of EL to scintillation photons can be defined in terms of the integrated scintillation and EL values as follows:

$$
\begin{gathered}
\text { ELvalue }=N_{E L} \times\left(E L_{\text {efficiency }}\right) \times(\text { PMTgainfactor }) \\
\text { Scintvalue }=N_{\text {scintillation }} \times\left(S_{\text {efficiency }}\right) \times(\text { PMTgainfactor }) .
\end{gathered}
$$

where $N_{E L}$ and $N_{\text {scintillation }}$ are the total number of EL and scintillation photons 
respectively, and the PMT gain factor is photon to volt conversion factor. Therefore the number of EL photons relative to the number of scintillation photons is given by:

$$
\frac{N_{E L}}{N_{\text {scintillation }}}=\frac{\text { Mean }_{\text {ELvalue }} \times S_{\text {efficiency }}}{\text { Mean } n_{\text {Scintvalue }} \times E L_{\text {efficiency }}}
$$

where $M e a n_{E L v a l u e}$ and $M e a n_{\text {Scintvalue }}$ are the Gaussian means of the integrated EL and scintillation distributions respectively. The number of scintillation photons relative to the number of ionization electrons is found from the ratio of $\mathrm{W}$-values $\left(N_{\text {scintıllation }} / N_{\text {ionization }}=W_{\text {oon }} / W_{s}\right)$. Therefore the number of EL photons produced for each ionization electron drifting through the entire EL region is given by:

$$
\left.E L \quad \text { gain }=\frac{\text { Mean }_{\text {ELvalue }} \times S_{\text {efficiency }} \times W_{\text {zon }}}{\text { Mean } n_{\text {Scintvalue }} \times E L_{\text {efficiency }} \times W_{s}} \quad \text { (photons/electron }\right) .
$$

After the corrections are applied the resulting curves are fit with Equation (3.4) to obtain the amplification and threshold parameters. 


\subsection{Detector Design}

The detector is housed in a Lesker stainless steel (SS) "T" chamber $32 \mathrm{~cm}$ long. It has three opening ports. Each port has a diameter of 6 " and terminates at an 8" Conflat (CF) flange (see Figure 3.3). To facilitate the production of EL light a cylindrical chamber insert was constructed based on a gas proportional scintillation counter (GPSC) design, Figure 3.5. The cylindrical insert is $10 \mathrm{~cm}$ long and has a $6 \mathrm{~cm}$ radius aluminium cathode, concentric to a cylindrical $0.5 \mathrm{~cm}$ copper anode held in place by 6 support rods that were machined out of "peek". Peek is a polyetherether ketone material which is a good insulator and can have a low outgassing rate. The choice to use a cylindrical geometry for the insert was to produce a well defined EL field and to eliminate the use of grid structures which tend to distort the field. The insert sits in the center of the T-chamber, held in place by 6 adjustable Teflon screws to ensure no electrical contact is made with the chamber walls. There are two feed through's located on port 3 to supply voltage to the anode-cathode system and allow for attachment of a preamp to measure the collected ionization signal. The two wires used to supply the voltage to the EL insert are encased in Teflon sleeves and a cylindrical Teflon "cap" (see Figure 3.4) was added to the anode end with the high voltage wire attached.

Two flanges with 6" glass viewports are mounted to axial ports, each one outfitted with a photomultiplier tube (PMT) to collect the scintillation and EL 


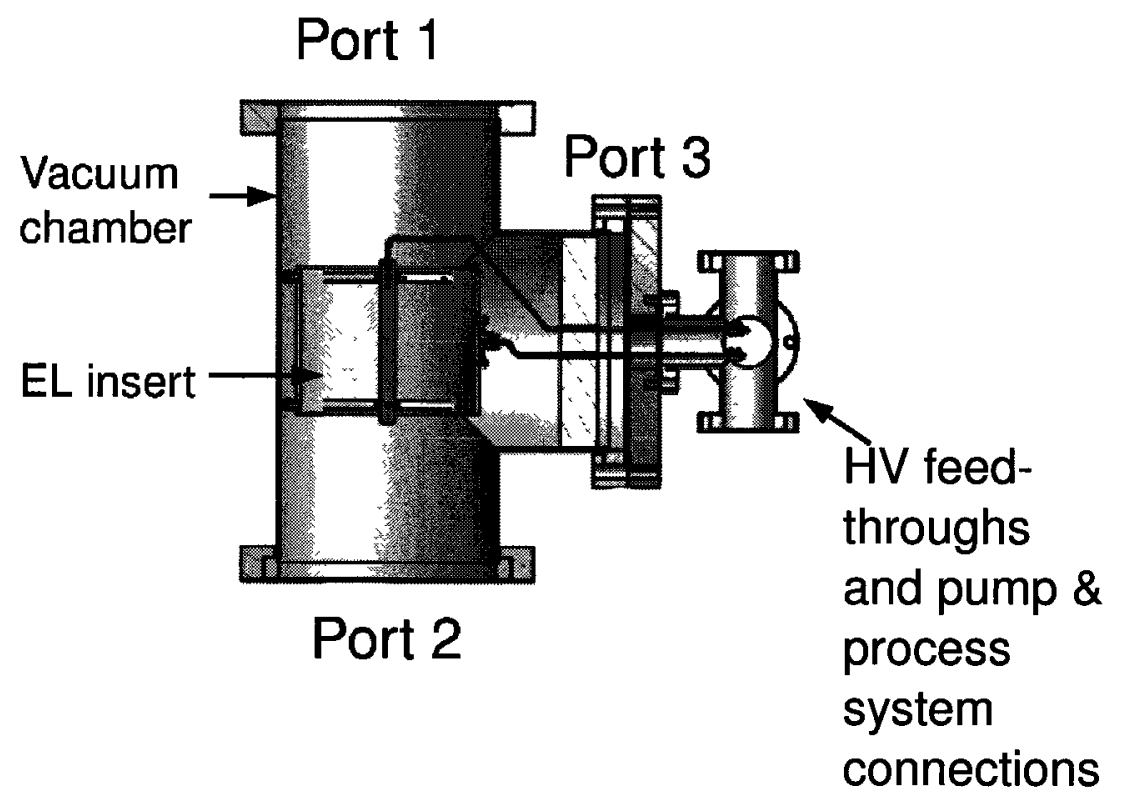

Figure 3.3: Lesker $\mathrm{T}$ vacuum chamber. Drawing by M. Bowcock.

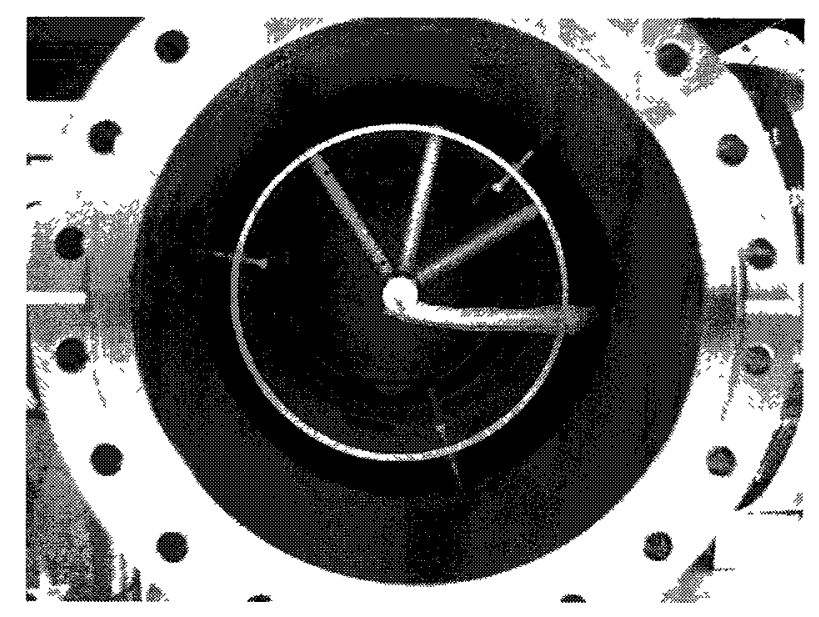

Figure 3.4: EL chamber insert with Teflon cap and Teflon insulated highvoltage wire. 
light. An ${ }^{241} \mathrm{Am}$ source is held in a hole machined out of the cathode positioned at $\mathrm{z}=0$ (see Figures 3.5 and 3.4. The ${ }^{241} \mathrm{Am}$ source emits a $5.49 \mathrm{MeV}$ alpha particle through $2 \pi$ steradians at a rate of $51.2 \pm 0.4$ (counts/s). Figure 3.6 is the $\alpha$ ionization spectrum of the ${ }^{241} A m$ source, obtained using an ORTEC 576A alpha spectrometer (Advanced Measurement Technology, Inc, 801 South Illinois Avenue, Oak Ridge, Tennessee) and an analogue to digital multi channel analyzer (ADMCA) (Amptek Inc., 14 De Angelo Drive, Bedford, MA. 01730 U.S.A.). The distribution was obtained for all angles of $\alpha$ particle emission, resulting in a Gaussian $\sigma /$ mean percent resolution of $\approx 1.6 \%(\approx 3.7 \%$ FWHM). Table 3.1 gives the relevant dimensions of the chamber and its components.

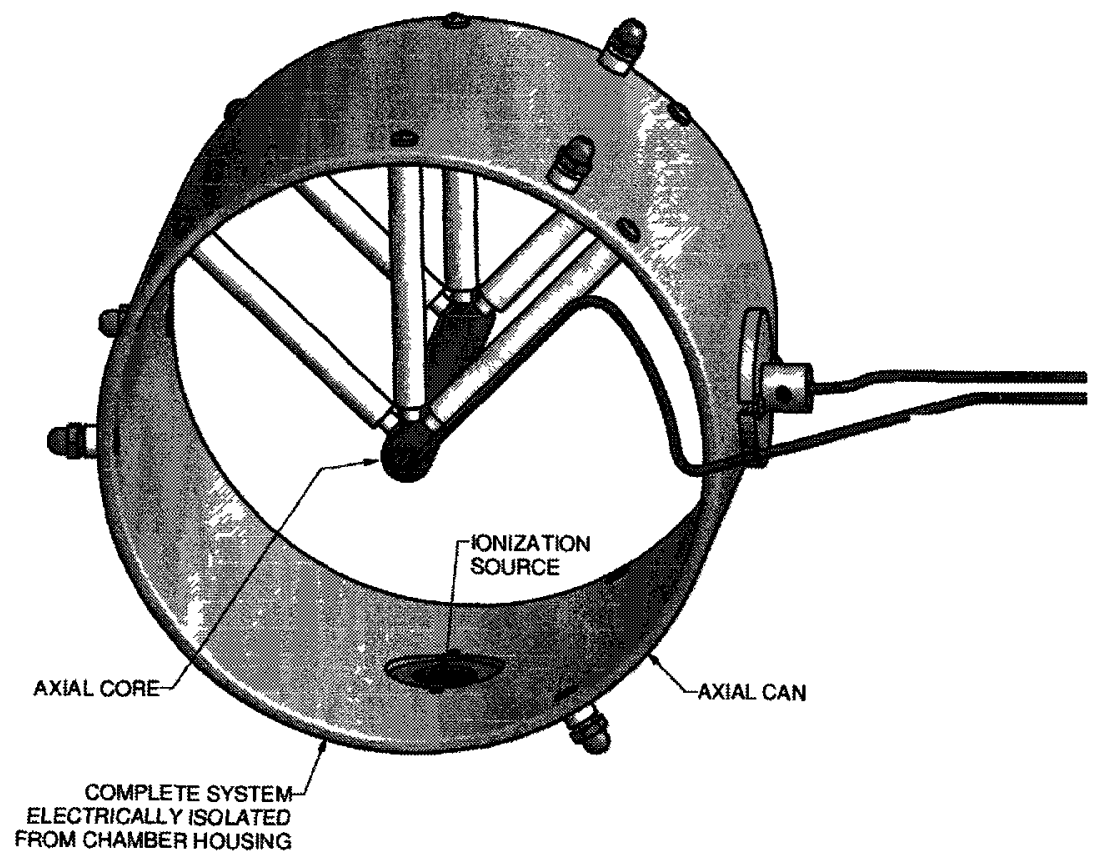

FIGURE 3.5: Drawing of cylindrical anode/cathode insert. Drawing by M. Bowcock. 


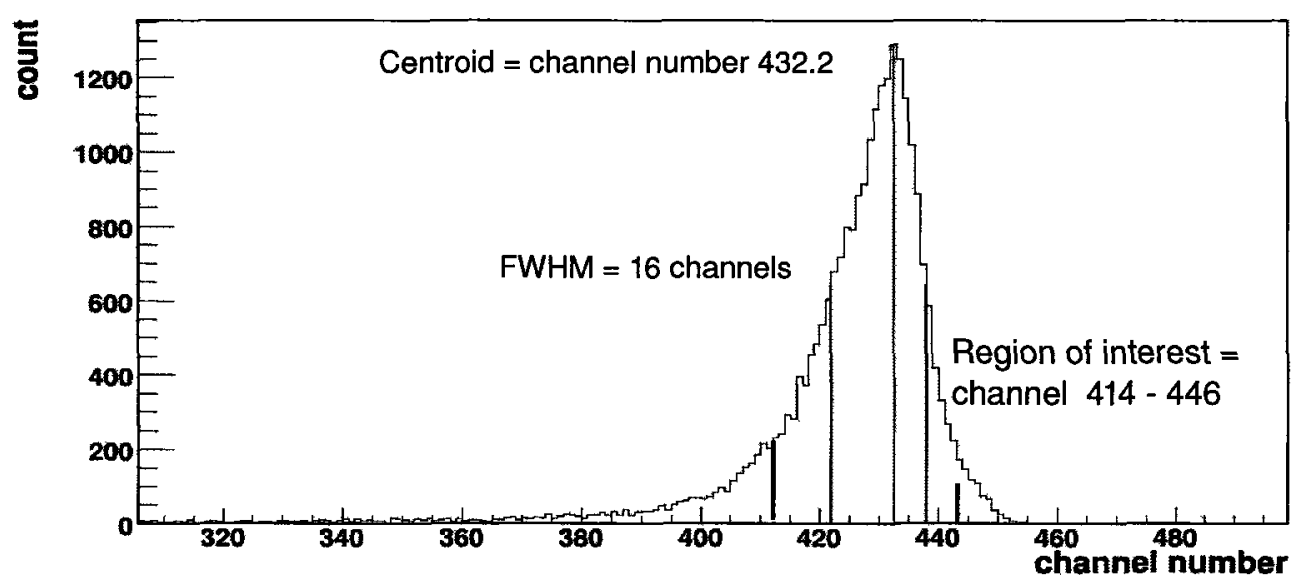

Figure 3.6: ADMCA spectrum of the ${ }^{241} A m$ source. Lines indicate the region of interest, FWHM, and the centroid.

\begin{tabular}{|c|c|}
\hline \multicolumn{2}{|c|}{ Detector Dimensions } \\
\hline chamber length & $35 \mathrm{~cm}$ \\
chamber radius & $7.5 \mathrm{~cm}$ \\
anode length & $10.4 \mathrm{~cm}$ \\
anode radius & $0.5 \mathrm{~cm}$ \\
cathode length & $10.2 \mathrm{~cm}$ \\
cathode radius & $6.0 \mathrm{~cm}$ \\
source diameter & $2.4 \mathrm{~cm}$ \\
PMT diameter & $12.7 \mathrm{~cm}$ \\
\hline
\end{tabular}

TABLE 3.1: Detector dimensions

The fully built detector was connected to a gas process system designed for purifying, filling and removing gases. A turbo-molecular pump was used to evacuate the chamber, reaching a final pressure of $1.1 \times 10^{-6} \mathrm{mb}$ before filling. Natural xenon gas, obtained from Praxair (1 City Centre Drive, Suite 1200 Mississauga Ontario) with a stated purity of $99.995 \%$, was used in making the measurements. 
While filling the chamber to 1 bar, the xenon was passed through a SEAS getter at an approximate flow rate of $\approx 50$ standard litres per minutes (slpm) to further eliminate electronegative impurities $\left(\mathrm{O}_{2}, \mathrm{H}_{2} \mathrm{O}, \mathrm{N}_{2}\right.$, etc. $)$ from the gas. SEAS (20020 Lainate (Milan) - Italy) getters are high temperature getters capable of reaching concentrations of electronegative impurities of less than $1 \mathrm{ppb}\left(\mathrm{O}_{2}\right)$. The getter works by the principle of adsorption, meaning, that the getter materials irreversibly trap contaminant gas molecules on their surface. No in-situ gas purification or purity monitoring was done once the chamber had been filled with gas. Xenon was removed from the chamber with cryogenic pumping using liquid nitrogen. The chamber was filled to an operating pressure of $1 \pm 0.001 \mathrm{bar}$, monitored with an Ashcroft 2089 digital pressure gauge, accurate to 0.05\%. All measurements were preformed at 1 bar, limited by the pressure rating of the glass viewports. The level of xenon purity is very crucial to the measurements. Levels of contaminant at the ppm level can alter the light producing and electron transport properties such as the drift velocity of the gas.

\subsubsection{EL Insert Design}

A number of considerations were made when designing the EL insert. The maximum cathode radius is constrained by the inner diameter of the T-chamber, where a radius of $6 \mathrm{~cm}$ was chosen, allowing a $\sim 1 \mathrm{~cm}$ gap between the chamber walls and 
the outer surface of the cathode. With the cathode radius fixed an anode radius had to be chosen which optimized the production of EL over a wide range of operating voltages with the constraint that all the emitted alpha particles should come to rest outside of the EL region. If alphas are allowed to enter the EL region each ionization electron will not produce the same amount of EL light because some electrons will drift through the entire region while others will only drift through a portion of the EL region. The ability to obtain optimal energy resolution relies on the accuracy by which electrons can be counted. In order to achieve a high accuracy using EL, each electron must produce the same amount of light and therefore drift over the same length in the EL region. This type of variance in the EL signal will degrade energy resolution and make EL gain measurements unreliable. Therefore in order to optimize the dimensions of the anode radius and the length of the insert, the range of the $5.49 \mathrm{MeV}$ alpha particle was also taken into account.

To determine the range of a $5.49 \mathrm{MeV}$ alpha particle in 1 bar of xenon gas a simple Monte Carlo simulation was performed using GEANT4. The simulation consisted of a cylindrical volume filled with xenon gas at a pressure of one bar. Each event emitted a single alpha particle with the desired energy into the gas volume. The simulation followed the $\alpha$ as it deposited its energy into the gas and recorded the range of the particle. The distribution obtained from the generation of 5000 events is shown in Figure 3.7. The distribution has a mean of $2.4 \mathrm{~cm}$ and 
an RMS of $0.438 \mathrm{~cm}$, with $99 \%$ of the events having a range less then $25 \mathrm{~mm}$ and $100 \%$ of the events less than $25.62 \mathrm{~mm}$. The range obtained for an alpha particle in 1 bar of xenon is in reasonable agreement with the NIST value of $2.33 \mathrm{~cm} \mathrm{[44].} \mathrm{In}$ determining the anode radius a conservative value of $3 \mathrm{~cm}$ was taken for the alpha range [45], requiring a maximum EL radius of $3 \mathrm{~cm}\left(R_{\text {max }}=R_{\text {cathode }}-R_{\text {alpha }}\right)$.

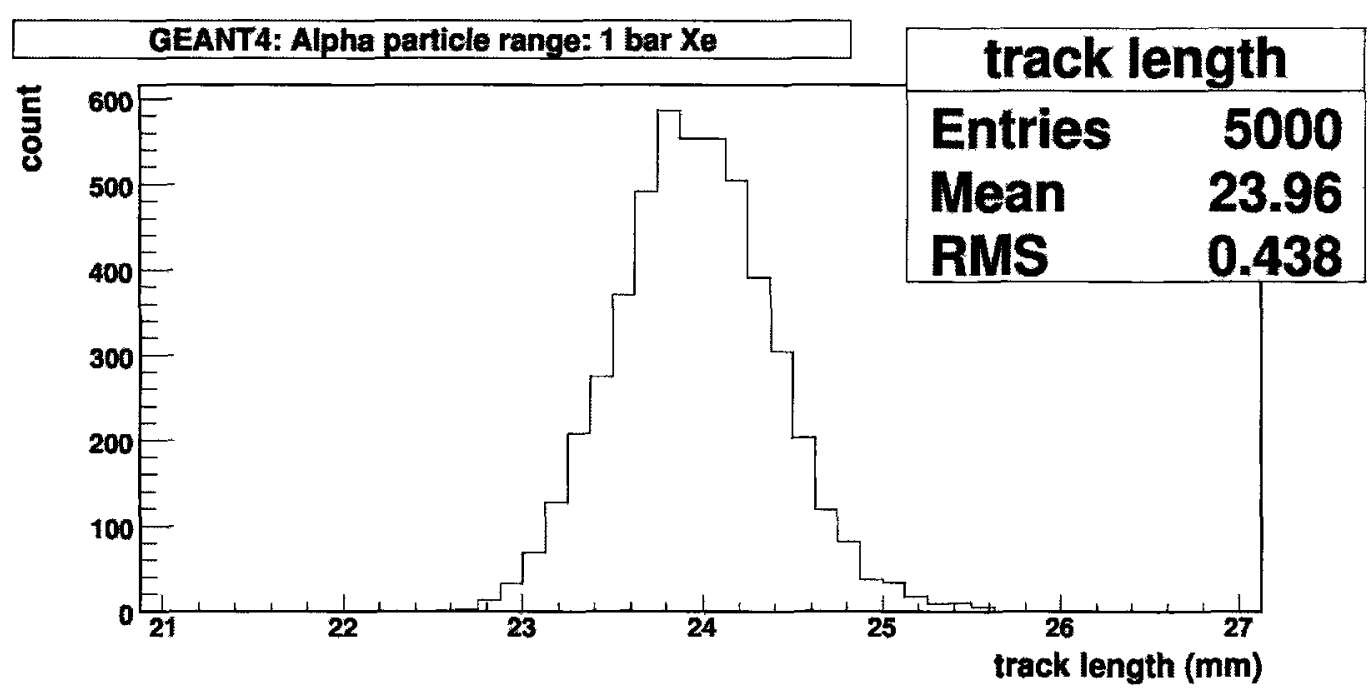

FIGURE 3.7: Distribution of ionization track lengths for alpha particles in 1 bar of xenon obtained from GEANT4.

Using the maximum EL radius $\left(r=R_{\max }\right)$ in Equations 3.2 and 2.14, and adopting the EL threshold value found by Monteiro et al. $\quad(B=0.83 \mathrm{kV} / \mathrm{cm}$-bar [3]) plots illustrating the dependence of $R_{E L}$ and $N_{E L}$ on anode radius were used to determine an optimum anode radius. Figure 3.8a shows a $R_{E L}$ optimization plot for voltages of $5.5,3.5$ and $2 \mathrm{kV}$, where the region of optimum anode radius is defined by the threshold for gas gain and the maximum allowable EL radius for $5.5 \mathrm{kV}$. Figure $3.8 \mathrm{~b}$ is the corresponding EL gain optimization plot. The figure 
shows that the chosen anode radius works for both considerations for the given voltages. Plots were made for a range of high voltages and it was determined that an anode radius of $0.5 \mathrm{~cm}$ resulted in good optical gain over a large range of operating voltages. The choice of $0.5 \mathrm{~cm}$ for the anode radius with the assumption that $\mathrm{B}=0.83(\mathrm{kV} / \mathrm{cm}$-bar $)$ results in an $\mathrm{EL}$ radius that extends past $3 \mathrm{~cm}$ for voltages higher then $6.1 \mathrm{kV}$. However, $3 \mathrm{~cm}$ is a very conservative number for the maximum EL radius since the maximum $\alpha$ track is expected to be $25.62 \mathrm{~mm}$ resulting in $R_{\max }=34.4 \mathrm{~mm}$. Assuming $R_{\max }=34 \mathrm{~mm}$ and B=0.83 (kV/cm-bar) in Equation (3.2) results in a voltage of $7.01 \mathrm{kV}$, indicating $\alpha$ particles do not enter the EL region for voltages below $7 \mathrm{kV}$.

The cylindrical field generated by the anode-cathode will have radial field lines with equipotential curves which are concentric to the anode. However the cathode is not a continuous surface since it has a $\sim 2.6 \mathrm{~cm}$ hole machined out of it where the ${ }^{241} A m$ source is positioned. Therefore the electric field produced will not be completely uniform. How much this non uniformity will affect the field lines was investigated using a FEMLAB simulation [45]. The results for a perfectly cylindrical cathode with a small hole removed had little to no effect on the field lines in the region of the hole. The field will also suffer from non-uniformitys at either end of the insert due to edge effects. The cathode extends $5 \mathrm{~cm}$ along the axis from the source center, the source diameter is $2.4 \mathrm{~cm}$, and therefore some of the alphas are emitted as far as $1.2 \mathrm{~cm}$ from the cathode center. Given that the 

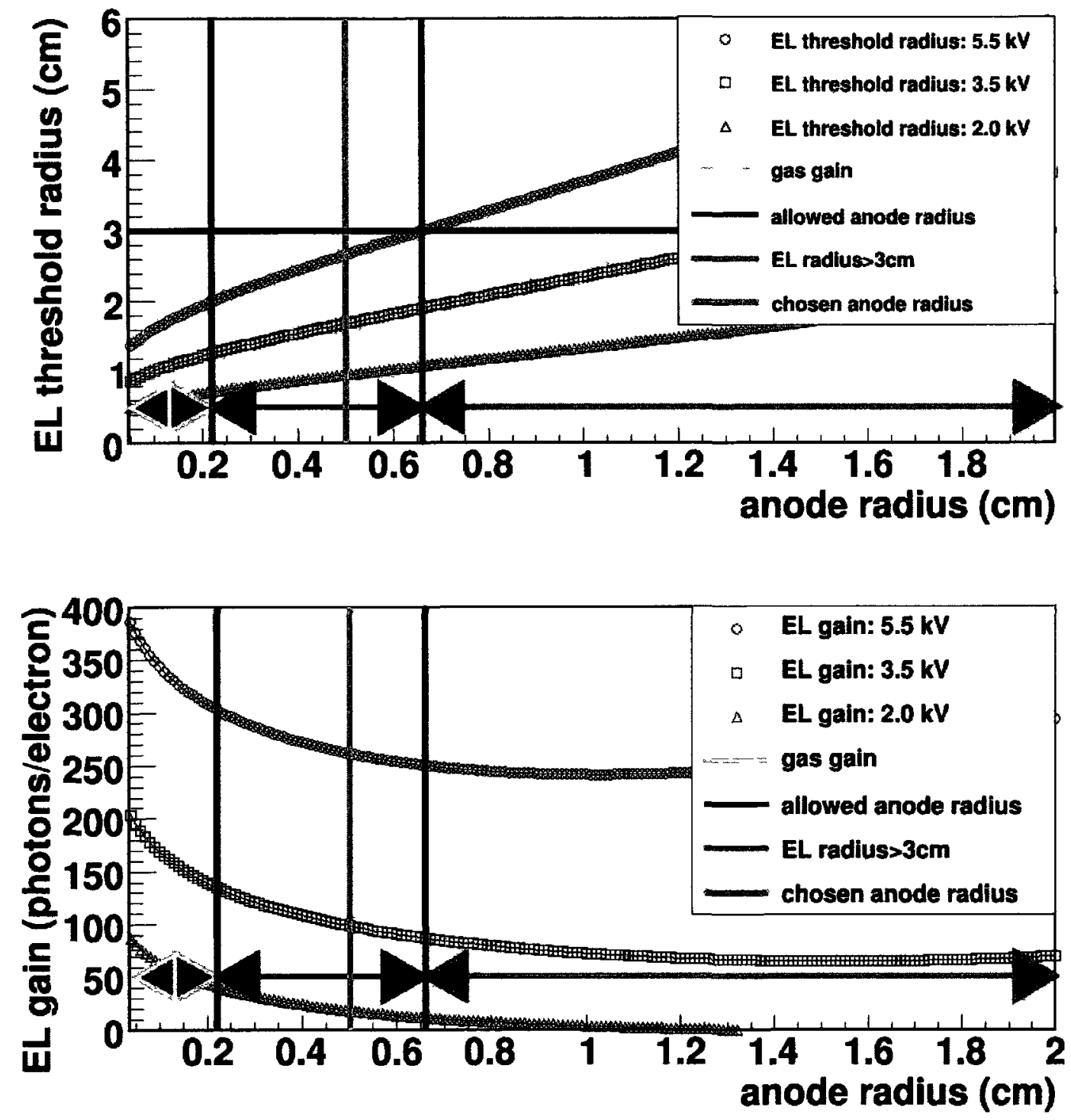

FiguRE 3.8: Optical gain and $R_{E L}$ dependence on anode radius: top: a) EL threshold plotted using Equation (3.2) with $\mathrm{B}=0.83(\mathrm{kV} / \mathrm{cm}-\mathrm{bar})[3]$, bottom:

b) EL gain determined by integrating Equation (2.14) from $\mathrm{a} \rightarrow \mathrm{r}=\mathrm{R}_{\max }$ and plotting as a function of a. $(\mathrm{B}=0.83(\mathrm{kV} / \mathrm{cm}-\mathrm{bar})[3])$.

maximum $\alpha$ length is $2.562 \mathrm{~cm}$ a uniform field is required extending at least $3.8 \mathrm{~cm}$ along the z-axis. Edge effects should not be present at a distance of $0.5 \mathrm{~cm}$ away 
from the edge, though this has not been investigated thoroughly. Therefore, $\alpha$ particles emitted in any direction, from any point on the source should experience a uniform electric field. However, if non-uniformitys are present, they can affect electron transport and EL generation. The combination of source position, angle of emission, and the geometry of the EL insert can result in alpha particles which hit the cathode wall and do not deposit all of their energy into the gas.

An ORTEC charge sensitive preamplifier was used to amplify the primary anode signal. The preamp was modified to allow voltage to be supplied through the preamp to both the anode and cathode of the EL insert. This was done to prevent breakdown that had been occurring between the high-voltage anode wire and the grounded chamber for voltages above $\sim 4000$ volts, limiting the maximum EL operating voltage to 4000 volts. With the modification, voltage of opposite polarity could be applied to the anode and cathode symmetrically. A negative bias was applied to the cathode and a positive bias applied to the anode through two Teflon insulated wires, while the chamber remained tied to ground. The voltage on both the anode and cathode could be kept below $4 \mathrm{kV}$ preventing electrical breakdown and allowed for operating at voltages up to $8 \mathrm{kV}$. 


\subsubsection{PMT Response Characterization \& Calibration}

The two PMTs (PMTA \& PMTB) used are 5" Electron Tubes model 9390QB (300 Crane Street, Sweetwater Texas 79556, USA) and are mounted to the viewports using Dow Corning Q2-3067 optical couplant. While these PMTs are capable of detecting UV light the glass viewports to which they are mounted do not efficiently transmit UV light. In order to collect the $178 \mathrm{~nm}$ UV light a thin film of wavelength-shifter (WLS) was deposited on two glass disks positioned within the xenon volume. The type of WLS chosen was Tetraphenyl-butadiene 4-1-1 (TPB) which was vacuum evaporated to a thickness of $\sim 0.2 \mathrm{mg} / \mathrm{cm}^{3}$ onto two $14 \mathrm{~cm}$ diameter, $1 / 4$ " thick glass plates. Each plate was placed $1.5 \mathrm{~cm}$ in front of either viewport, with the TPB facing the center of the detector. This way any photons incident on the viewports will first hit the TPB and get shifted from UV to the visible spectrum. TPB emits isotropically with a high conversion efficiency, and a fast fluorescence time constant of $1.9 \mathrm{~ns}$ [46].

The calibration of the PMTs was achieved by measuring each tube's response to the emission of a single photo-electron (SPE) from the photocathode, where the (SPE) spectrum of each tube was obtained for a range of PMT voltages. To resolve the SPE specta and calibrate the PMTs, an ORTEC 143B charge sensitive preamplifier was used in conjunction with an ORTEC 490 spectroscopic amplifier (Advanced Measurement Technology, Inc, 801 South Illinois Avenue, Oak 
Ridge, Tennessee), an AMPTEC pocket 8000A analogue to digital multichannel analyzer (ADMCA), and pulse generator. The raw signal from the PMTs was fed into the preamp and then to the spectroscopic amplifier and to the ADMCA. The output of all components was monitored on an oscilloscope. A pulse from the pulse generator was sent to the test input of the preamp and was used to calibrate the amount of charge in the SPE peak. Each spectrum was taken over a over run period of 5 minutes. The setup is illustrated in Figure $3.9 \mathrm{a}$, and $3.9 \mathrm{~b}$ depicts the circuit used for calibration.

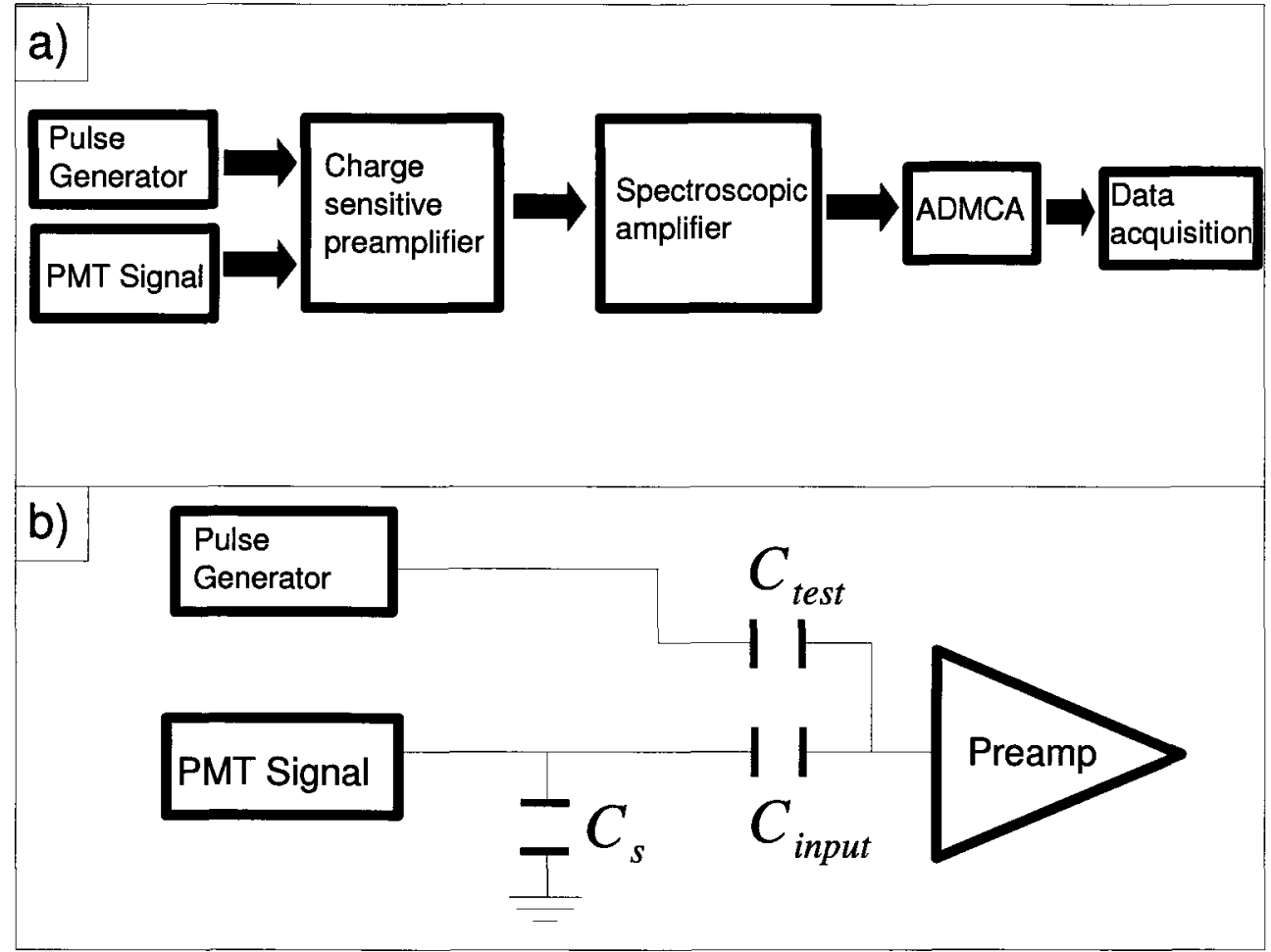

FiguRE 3.9: a) System setup used for PMT calibration.

b) Calibration circuit. 
To obtain the charge calibration of the SPE peak, a pulse generator is used to send a known voltage pulse $\left(\mathrm{V}_{P}\right)$ to the preamp's test input. Then using the known capacitance of the test input $\left(\mathrm{C}_{t e s t}\right)$, and the voltage of the pulse $\left(\mathrm{V}_{P}\right)$, the amount of charge input to the preamp from the pulser is determined by $Q_{P}=$ $C_{t e s t} V_{P}$. Using this charge and the pulser's peak mean channel number obtained from the fit a charge per channel conversion for the ADMCA spectrum is found. Then from the charge per channel conversion and the Gaussian mean obtained from the fit of the SPE distribution, the amount of charge being input to the preamp by the PMT is found $\left(\mathrm{Q}_{I N}\right)$. The preamp was modified by adding a 0.98 $\mathrm{pF}$ capacitor in series with the main input, $\left(\mathrm{C}_{I N}\right)$. This was done to allow the voltage equivalent of the PMT to be determined using $Q_{I N}=C_{I N} V_{P M T}$.

Each PMT has an associated stray capacitance from the dynode chain as well as an additional stray capacitance of the coaxial connector feeding the preamp. The two stray capacitances were measured and added in parallel to give a total stray capacitance $\left(C_{S}=C_{P M T}\right)$ of $50 \mathrm{pF}$. The charge output by the PMT can be found from $Q_{P M T}=C_{P M T} V_{P M T}$ using the total stray capacitance and the previously defined $V_{P M T}$. Using the charge one can find the total number of electrons output per single electron emitted from the photocathode, ie. the gain of the tube. Figure 3.10 is a typical spectrum taken with the above setup while Figure 3.11 showing the SPE peak fit results. The fitting function was a Gaussian distribution convoluted with an exponential background function. Each SPE peak 
has a count on the order of 100000 , resulting in a high statistical accuracy for the measurements. While the statistical error of the measurements is low the systematic error is believed to dominate and gain values are presented including systematic errors. The systematic error introduced to the measurements are due to uncertainties on the value of the capacitors used in the calculations along with the error in the power supply readings. The voltage error was taken to be half of the last digit on the supply readout while the capacitance errors were taken as being $1 \%$ of the given value. The errors were then determined from error propagation.

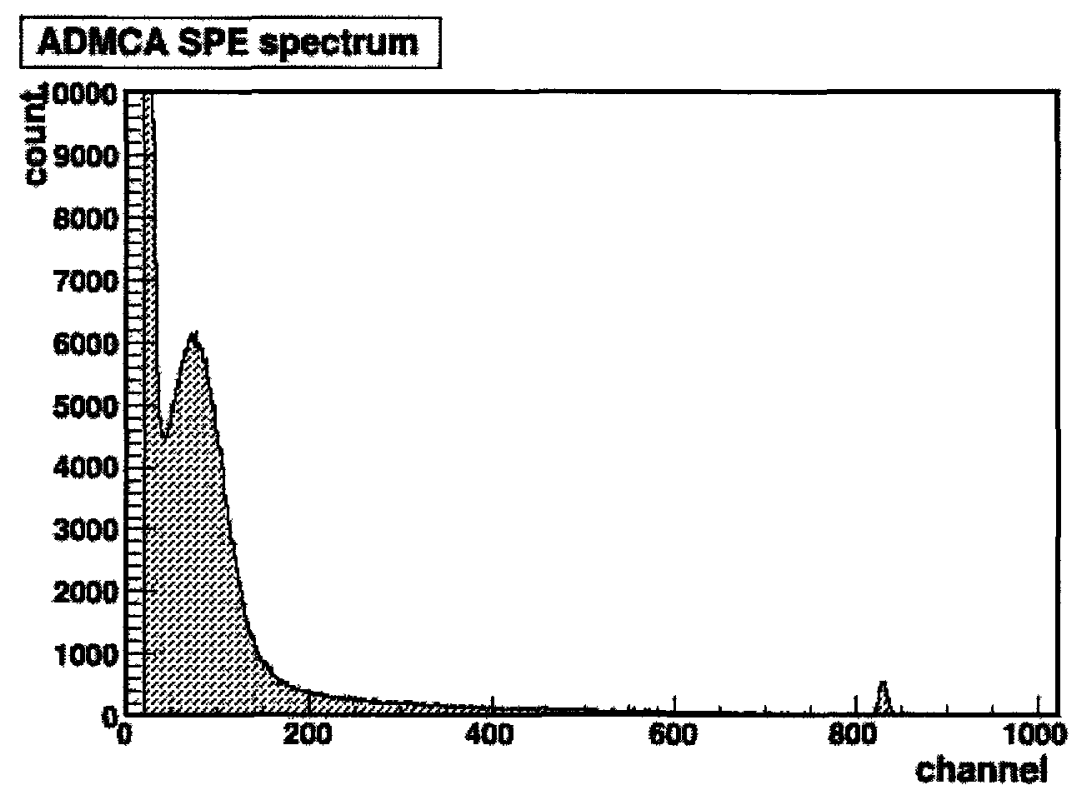

FIGURE 3.10: PMTB 1.3 kV: ADMCA spectrum showing an SPE peak centered at channel 75 and a pulse generator peak located at channel $\approx 830$

Gain measurements were recorded for each PMT over a range of operating voltages and a plot of the gain versus applied voltage for each PMT is shown in 


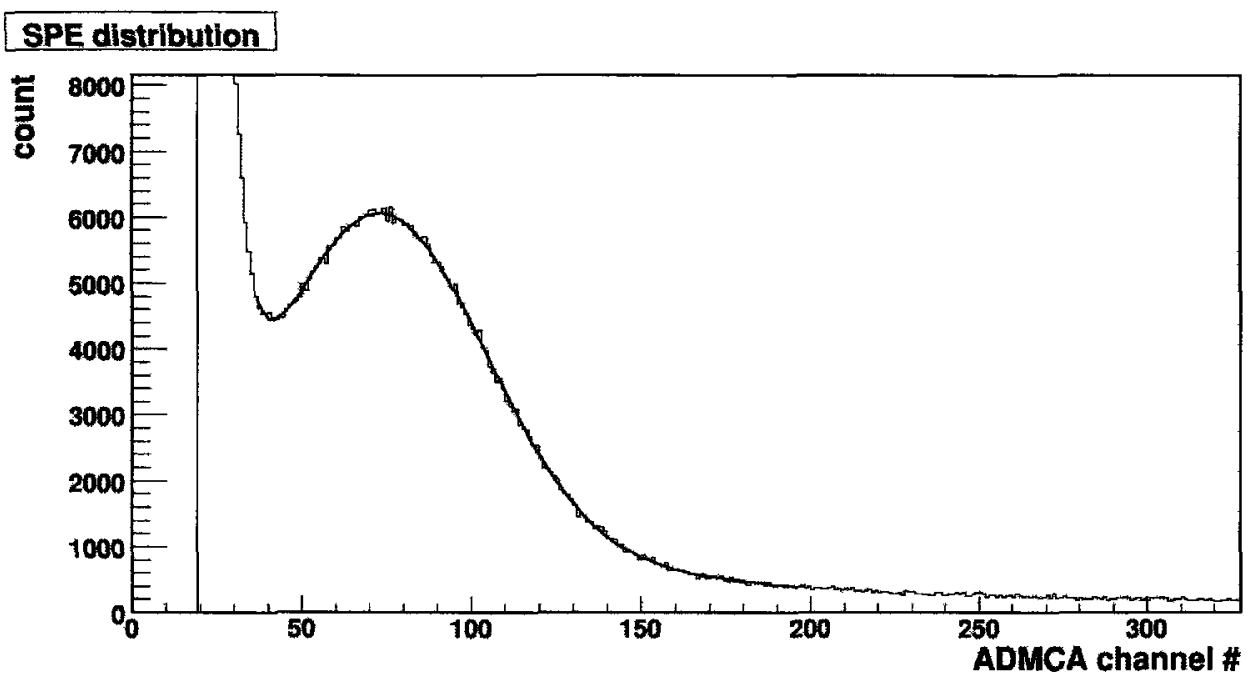

FIGURE 3.11: SPE spectrum.

\begin{tabular}{|c|c|c|c|c|}
\hline \multicolumn{5}{|c|}{ PMT gain (e's) } \\
\hline PMT voltage & PMTA gain & PMTA sys error & PMTB gain & PMTB sys error \\
\hline 1200 & $7.75 \times 10^{5}$ & $9.04 \times 10^{4}$ & $5.46 \times 10^{5}$ & $6.39 \times 10^{4}$ \\
1300 & $1.65 \times 10^{6}$ & $1.89 \times 10^{5}$ & $1.10 \times 10^{6}$ & $1.26 \times 10^{5}$ \\
1400 & $3.13 \times 10^{6}$ & $3.56 \times 10^{5}$ & $2.21 \times 10^{6}$ & $2.51 \times 10^{5}$ \\
1500 & $6.21 \times 10^{6}$ & $7.06 \times 10^{5}$ & $4.29 \times 10^{6}$ & $4.89 \times 10^{5}$ \\
1600 & $1.07 \times 10^{7}$ & $1.21 \times 10^{6}$ & $7.40 \times 10^{6}$ & $8.41 \times 10^{5}$ \\
\hline
\end{tabular}

TABLE 3.2: PMT gain.

Figure 3.12. Individual gain and error values given in Table 3.1.2. The gains are approximately logarithmic over the voltage range sampled, where the two PMTs differ in response with PMTA having a higher gain than PMTB by a factor of $\sim 1.5$.

In making measurements, it is desirable to have the response for both PMTs equalized so that the detector response is consistent for tracks emitted towards either PMT. This also allows for a more accurate measurement of the $\alpha$ track 


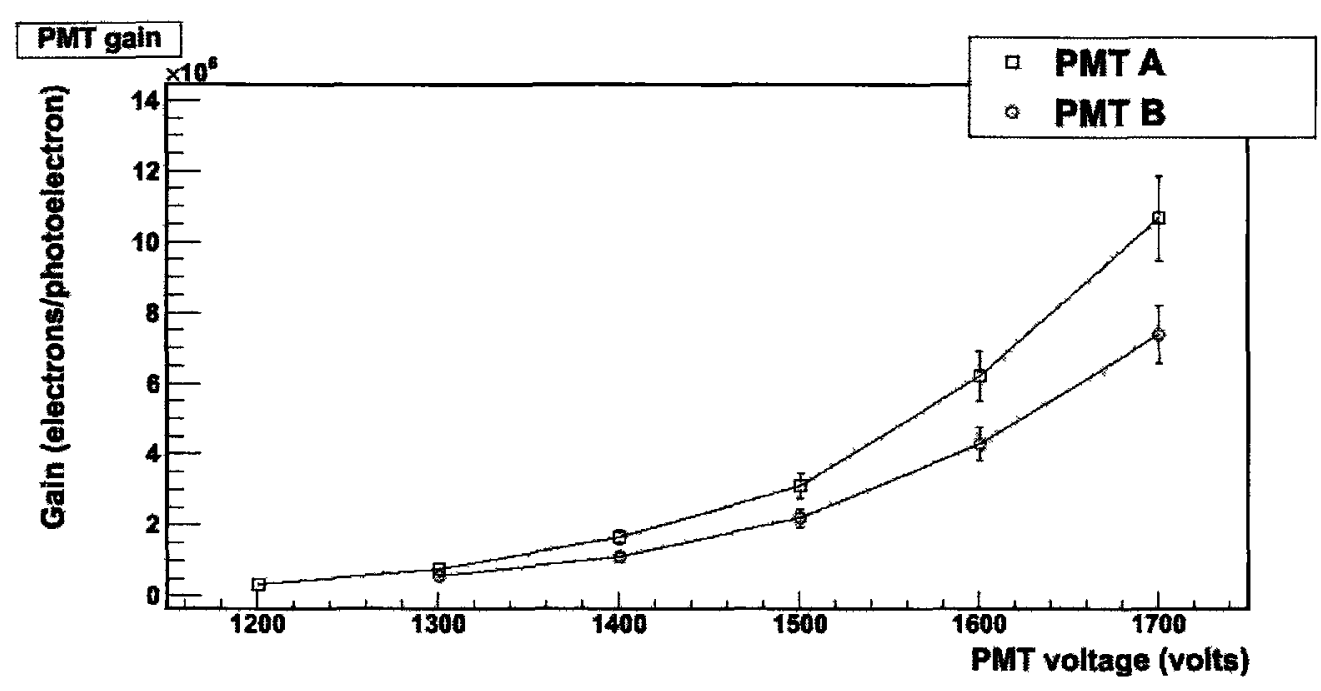

FIgure 3.12: PMT gain including systematic errors vs. PMT voltage: Top curve: PMTA, Bottom curve: PMTB

angle. Since there is no absolute calibration of the digitized data, perfect response matching is not required. PMT response equalization is accomplished by matching the gain of the tubes. The gain of a PMT is given by $G=V^{n}$ where $\mathrm{G}$ is the gain, $\mathrm{V}$ the voltage, and $\mathrm{n}$ is the number of dynodes, in this case 10. An assumption of linear gain is obviously incorrect but it should give a reasonable starting voltage in determining the equalization. Assuming a linear gain relation a PMT voltage of 1240 volts is obtained for tube A given tube B is at 1300 volts. The voltage of 1240 was used as a starting point then manually adjusted until good agreement was obtained between the raw PMT signals monitored on the scope. Figure 3.13 shows the individual PMT scintillation distributions after gain matching. The Gaussian means agree within $3 \%$. PMT operating voltages of 1.252 and $1.300 \mathrm{kV}$ were chosen for PMTA and PMTB respectively. As the insert anode/cathode voltage is 
increased the EL pulse becomes very large compared to the primary scintillation pulse. If the PMT voltages are set too high the sensitivity to the scintillation pulse at high voltages becomes poor. PMT operating voltages of $1.252 \mathrm{kV}$ and 1.300 $\mathrm{kV}$ allow for sensitivity to the scintillation pulse over the entire voltage range and are well below the saturation point.

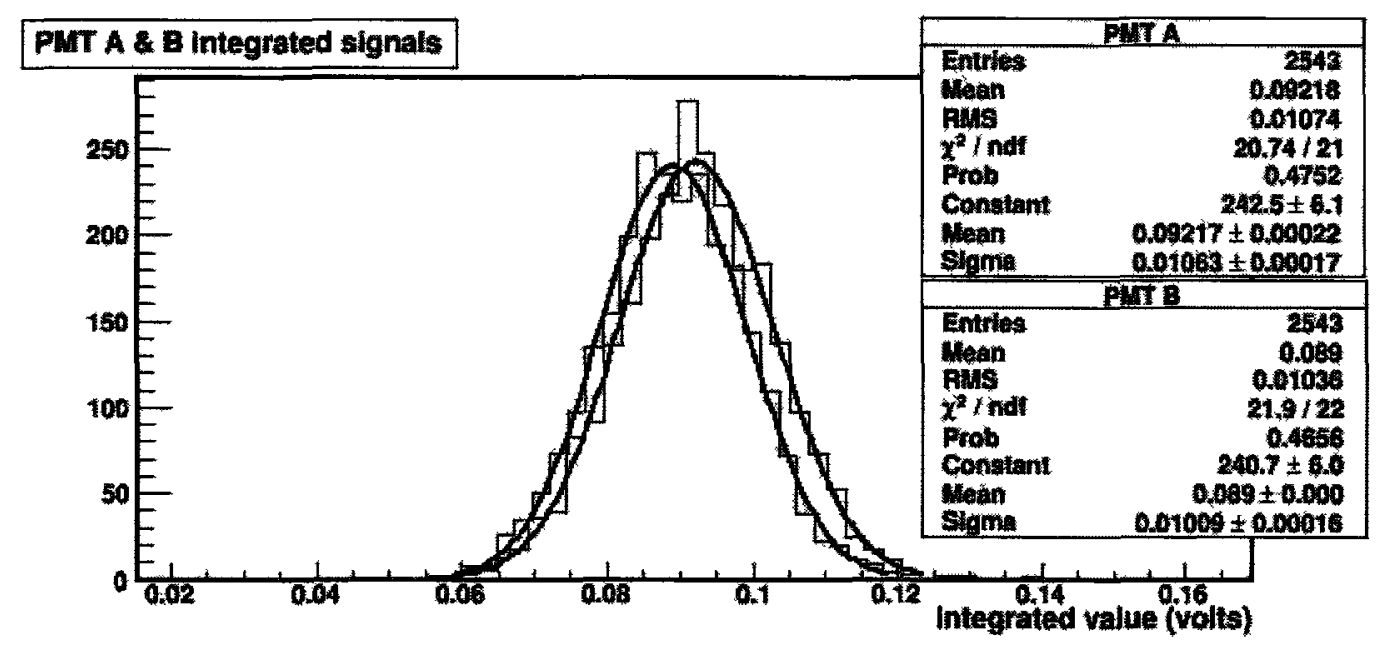

FIGURE 3.13: Integrated scintillation amplitudes for PMT's A\&B. Gaussian fits result in mean values which agree within $3 \%$.

\subsection{Data Acquisition and Detector Output}

The raw signal from each PMT along with the EL insert preamplifier signal were input to a Tektronics TDS 3054 oscilloscope (14150 SW Karl Braun Drive, Beaverton, OR). The scope was used to monitor the signals and acquire data traces of the signals for each event. A LabView program was written which acquired the 
data from a number of events, sequentially writing it to a data file. The scope was set to record over a period of $100 \mu$ s using 10000 data points resulting in a time binning of $10 \mathrm{~ns} / \mathrm{bin}$. Triggering was done on the anode signal for voltages of $0.5 \mathrm{kV}$ and above. For lower voltages the scintillation pulse was used for the trigger as the anode signals were too weak and unstable. The scope voltage window was set run by run to optimize the EL pulse in the acquisition window. The temperature was also monitored at three points, one at each PMT base and the third one at the PMT power supply, with temperature readings taken for each event recorded. Data were acquired over a set of drift field voltages ranging from $(0-8) \mathrm{kV}$ in 250 volt intervals up to $2 \mathrm{kV}$ then in 500 volt intervals from (2-8) $\mathrm{kV}$. Each run consisted of obtaining greater than 15000 events at a particular drift field voltage. The PMT's power supplies were set to voltages of 1253 and 1300 volts for tubes $\mathrm{A}$ and $\mathrm{B}$ respectively, remaining constant to within \pm 0.5 volts over each run-time. At the beginning and end of each run measurements of PMT gain and the average number of primary scintillation photons reaching the PMTs were obtained to monitor the detector's response between each run.

The combination of the cylindrical drift field with the $\theta-\phi$ dependence of the ionization tracks result in raw data traces which depend on these parameters as different track orientations result in different time and amplitude characteristics for the acquired data pulses. Figures 3.14a,b illustrate the $\theta$ and $\phi$ dependence of the alpha tracks in relation to the source and detector. The $\phi$ angle is defined to 
be $0^{\circ}$ when the $\alpha$ track is pointing directly at PMTA and $180^{\circ}$ when the $\alpha$ track in pointing directly at PMTB. The emission of alpha particles through $2 \pi$ steradians follows a $(1-\cos \theta)$ probability distribution, giving the highest probability to ionization tracks emitted nearly parallel to the surface of the source $\left(\theta \sim 90^{\circ}\right)$, and zero probability for ionization tracks emitted completely perpendicular to the sources surface $(\theta=0)$. Parallel tracks produce larger anode signals because all charge produced drifts through the entire anode/cathode separation, whereas perpendicular tracks distribute charge radially with the majority of the charge only drifting over a fraction of the total separation, inducing smaller currents. The arrival time of the mean of the charge distribution corresponds to the inflection point of the anode rise time, and is approximately equal to the time of the EL maximum for parallel tracks. The time required to drift the charge to the EL region is dependent on track angle. This results in different timings for the amplitude and duration of the EL pulse and the pulse onset relative to the scintillation pulse. PMT and anode data traces obtained at a voltage of $3 \mathrm{kV}$, displayed in the form of histograms, are shown in Figures $3.15 \mathrm{a} \& 3.15 \mathrm{~b}$. The traces correspond to approximately perpendicular and parallel alpha tracks respectively. After drifting from the cathode to the EL region, all the charge produced by a parallel track reaches the EL region at approximately the same time, this produces large amplitude EL pulses which have a short duration (see Figure $3.15 \mathrm{~b}$ ). Charge produced at the end of perpendicular tracks has a much shorter distance to drift before reaching the 
start of the EL region, resulting in shorter onset times for the EL pulses (see Figure $3.15 \mathrm{a})$. The charge is distributed along the track, sequentially drifting through the EL region, producing smaller amplitude EL pulses over a longer duration (see Figure 3.15a). Parallel and perpendicular tracks are the two extremes of alpha particle emission in the detector, Figure 3.16 shows a collection of traces obtained for $\theta$ ranging from $0^{\circ}$ to $90^{\circ}\left(0^{\circ}<\theta<90^{\circ}\right)$. Depending on the $\phi$ angle of emission some percentage of the alpha particles will hit the side of the cylindrical cathode depositing all or some fraction of its energy, reducing the scintillation light yield and producing less charge able to drift to through EL region, greatly reducing the EL yield. Events that hit the cathode and deposit energy producing less light are referred to as "range outs". Alpha tracks with $\theta \approx 90^{\circ}$ and $\phi \approx 90^{\circ}$ or $180^{\circ}$ are examples of range out events. 


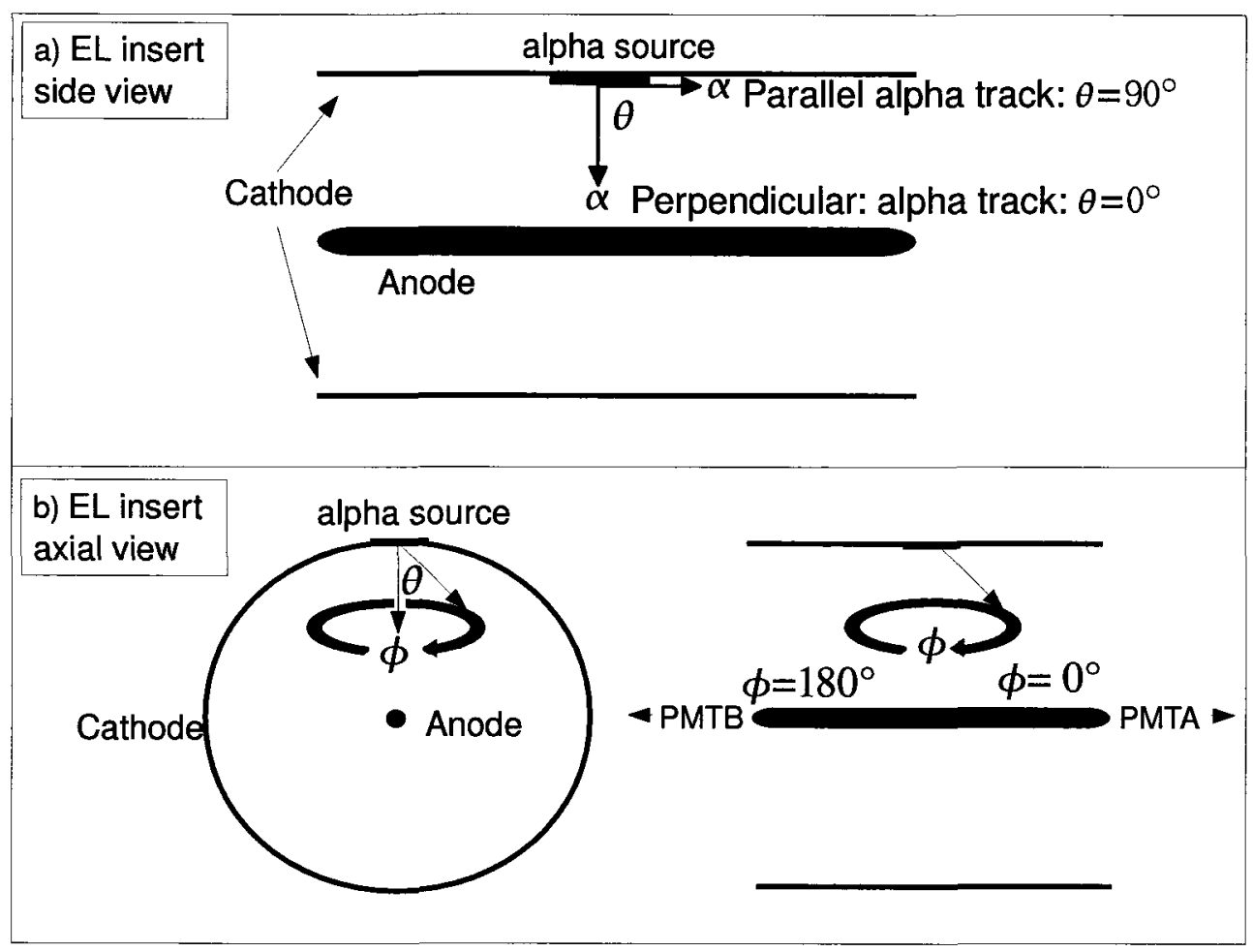

Figure 3.14: Ionization track orientation $\theta \quad \& \quad \phi$ dependence. a) Side view of EL insert. b) Axial view of EL insert. 

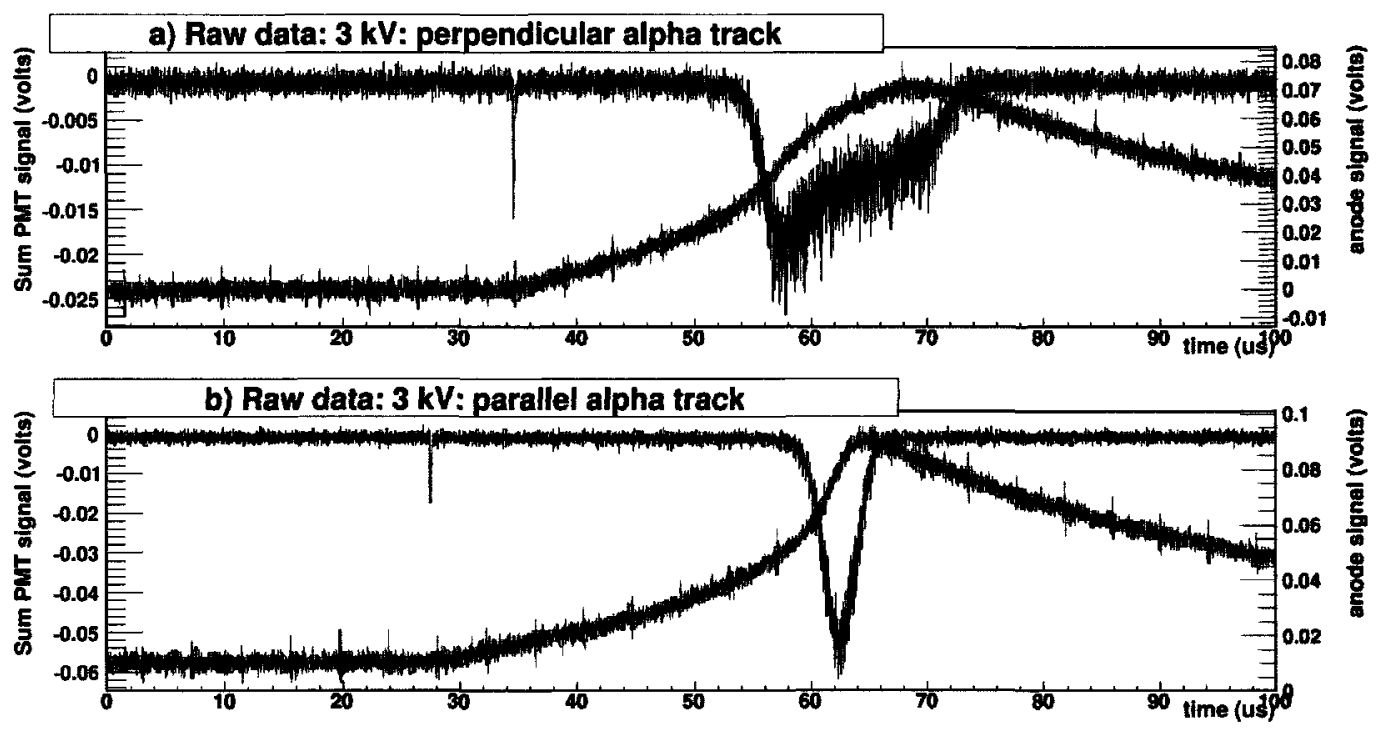

Figure 3.15: a) Perpendicular ionization track: Raw PMT signal shown in black. Raw anode signal shown in red. b) Parallel ionization track: Raw PMT signal shown in black. Raw anode signal shown in red.

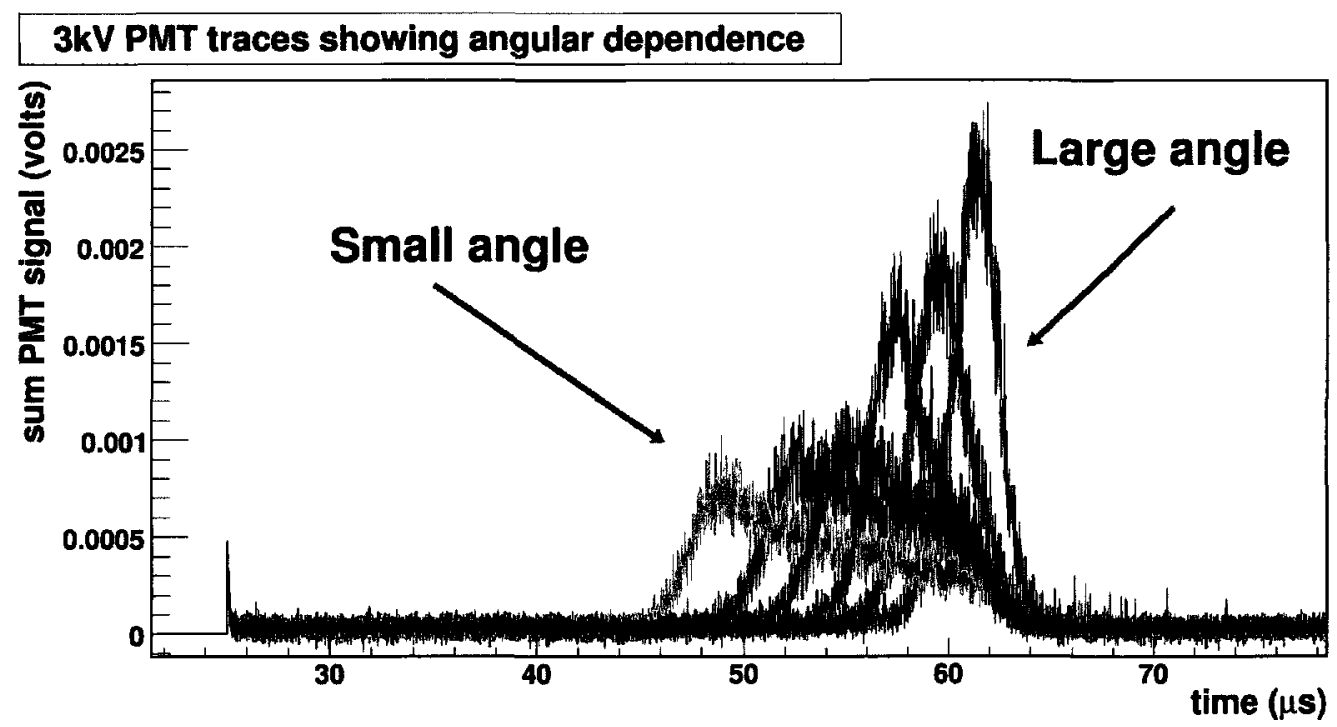

FIGURE 3.16: Raw PMT traces illustrating angular dependence. 


\section{Chapter 4}

\section{Monte Carlo Chamber Simulation}

Monte Carlo (MC) studies were performed in order to characterize the detector's expected response and output. The main purpose of the MC studies was to obtain the efficiency correction factors used in Equation (3.9). A detailed GEANT4 application (for details on GEANT4, see [47]) was constructed which simulated the interaction of $5.49 \mathrm{MeV}$ alpha particles within the $\mathrm{Xe}$ of the detector and was set to record the details of position, time, and energy deposited for the ionization tracks and the output of primary scintillation light. GEANT4 efficiency simulations were run which determined the point by point light collection efficiency for the light emitting regions of the detector. The chamber geometry was modelled as a cylinder excluding the "T" portion containing the electrical feed-throughs and vacuum connections. All other chamber and component dimensions were set to match the 
real detector dimensions. Figure 4.1 depicts the GEANT4 representation of the detector used in the simulation where the red, green and blue lines are the $\mathrm{x}, \mathrm{y}$, and z-axes respectively, and the red disks indicate the TPB WLS, blue are the glass plates that TPB is deposited on, with the EL insert in purple, and the Teflon cap in white. A TPB fluoresence time of 1.9 ns with a conversion efficiency of $100 \%$ were used as the WLS input parameters. A $\mathrm{C}++$ program was written which used the ionization tracks produced by GEANT4 to simulate the resulting EL pulses. The simulation of the PMT traces was of secondary importance but acted to check the consistency of the expected to measured detector response. The simulation details and results will now be discussed.

\subsection{Shadowing \& Chamber Efficiency}

Scintillation and EL light are emitted isotropically, and due to solid angle and shadowing effects from internal detector components the amount of light that reaches the two PMT faces is greatly reduced from the amount produced by either scintillation or EL. To determine the overall solid angle, shadowing due to specific detector components, and the individual efficiencies of spatial coordinates in the regions where light is produced, $100 \mathrm{k}$ photons were produced isotropically at representative points throughout the detector (photon bombs) and the simulation was set to record the spatial distribution of where the photons terminated 


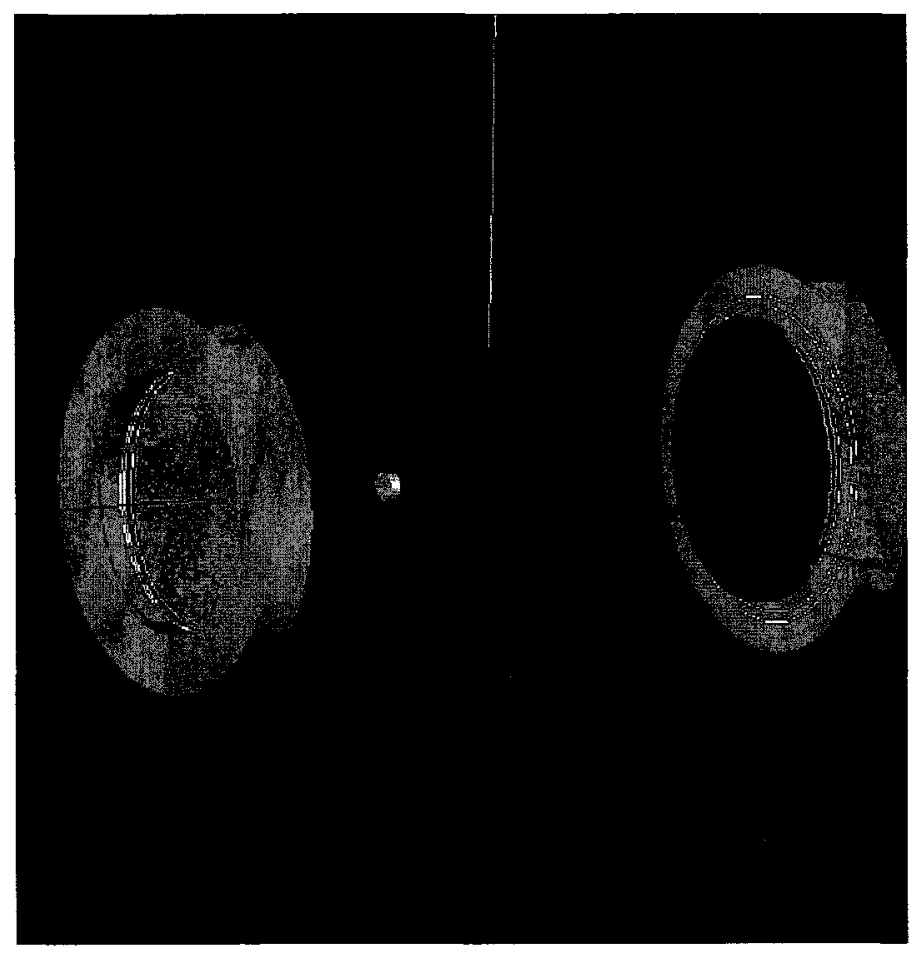

FIGURE 4.1: GEANT representation of EL test chamber. The vacuum chamber walls and PMTs are not shown. Axis colours: Red $=\mathrm{x}$, Yellow=y, Blue=z

in the detector. Define the solid angle to be the fractional number of generated photons that reach the PMT faces, and anode shadowing as the fractional number of generated photons which hit a particular structure. The collection efficiency at a particular point is then simply the ratio of the number of photons that hit either PMT face to the total number generated, while total solid angle and shadowing effects are determined from the distribution of photon hits on particular chamber components. The shadowing and efficiency simulation consisted of generating the $100 \mathrm{k}$ photons emitted isotropically at various points in the $y-z$ plane of the detector. The basic scheme is depicted in Figure 4.2a. The photon generation 
points were chosen to cover a 2-D region of the detector where scintillation and EL light would be produced. Positions were chosen at 33 locations along the z-axis between $\mathrm{z}=-4 \mathrm{~cm}$ and $\mathrm{z}=4 \mathrm{~cm}$ at $0.5 \mathrm{~cm}$ intervals, and 20 points at $0.275 \mathrm{~cm}$ intervals along the $\mathrm{y}$-axis spanning the $5.5 \mathrm{~cm}$ radial distance between the anode and cathode.

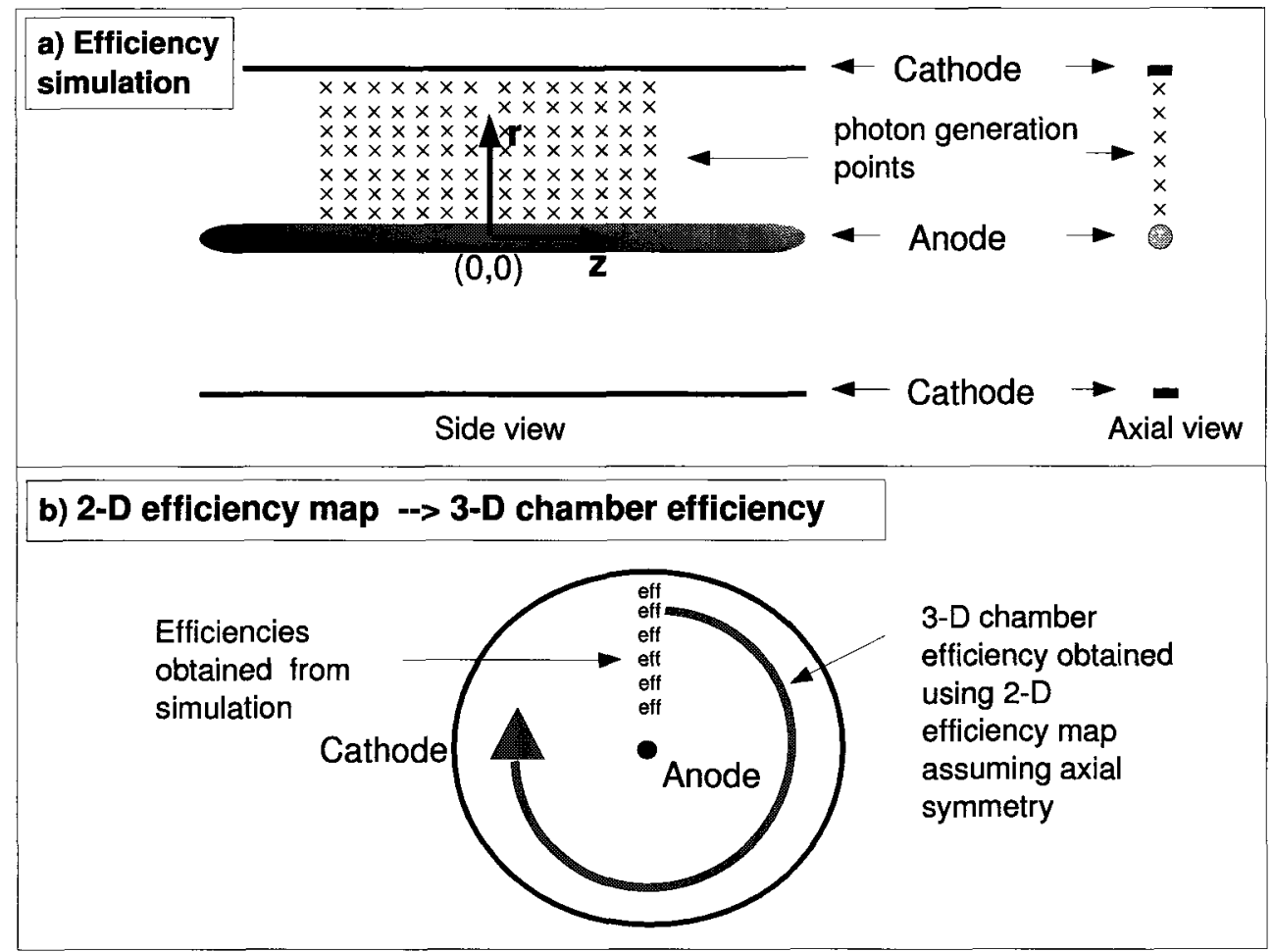

Figure 4.2: a) Depiction of efficiency simulation. b) Conversion of the 2-D efficiency map into a 3-D efficiency distribution.

The efficiency response can be broken into two independent regions, one corresponding to the region of the detector where scintillation light is produced (scintillation region) and the other corresponding to the region where EL light is 
produced (EL region). All primary scintillation light will be produced in the radial range $(3<\mathrm{r}<6) \mathrm{cm}$ where shadowing from the anode is minimal and solid angle coverage is dependent on the $\theta$ and $\phi$ of the track. EL will be produced in the radial range, $(0.5<\mathrm{r}<3) \mathrm{cm}$, resulting in large shadowing effects from the anode, especially for small radial distances. Efficiency distributions for the scintillation and EL regions are given in Figures 4.3 and 4.4 respectively. For comparison, the figures also show distributions obtained corresponding to the chamber without the EL insert. The mean efficiencies obtained for the chamber including the EL insert are $2.5 \%$ and $0.97 \%$ for the scintillation and EL regions respectively. In comparing the mean of the scintillation efficiency distributions with and without the EL insert, there is a $\approx 1 \%$ difference, indicating the addition of the EL insert has little effect on the solid angle efficiency for light emitted in the scintillation region. In considering the EL region efficiencies there is a $\approx 57 \%$ difference in the means obtained with and without the EL insert, the distributions are also quite different in shape. The distribution including the insert displays a low valued tail, with the events making up this portion of the distribution due to photon bombs that were generated very near the anode where shadowing is the highest. Anode shadowing distributions are shown in Figure 4.5, which gives the number of generated photons which are absorbed by the anode for photon bombs generated in the scintillation and EL regions. The reduction of solid angle coverage and added photon absorption due the addition of the insert structure is the reason for 
the $\sim 57 \%$ loss in the average chamber efficiency for the EL region.

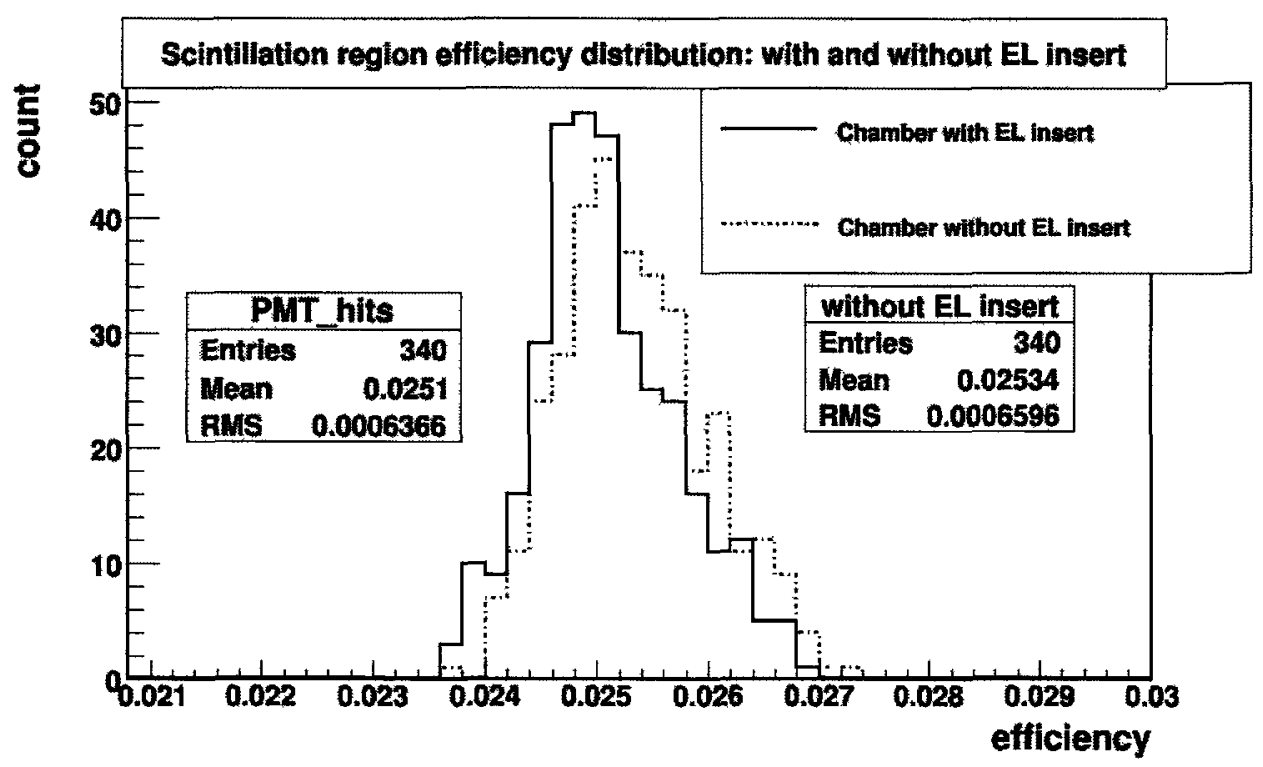

FIGURE 4.3: Light collection efficiency distributions corresponding to the scintillation region. Obtained with and without the EL insert.

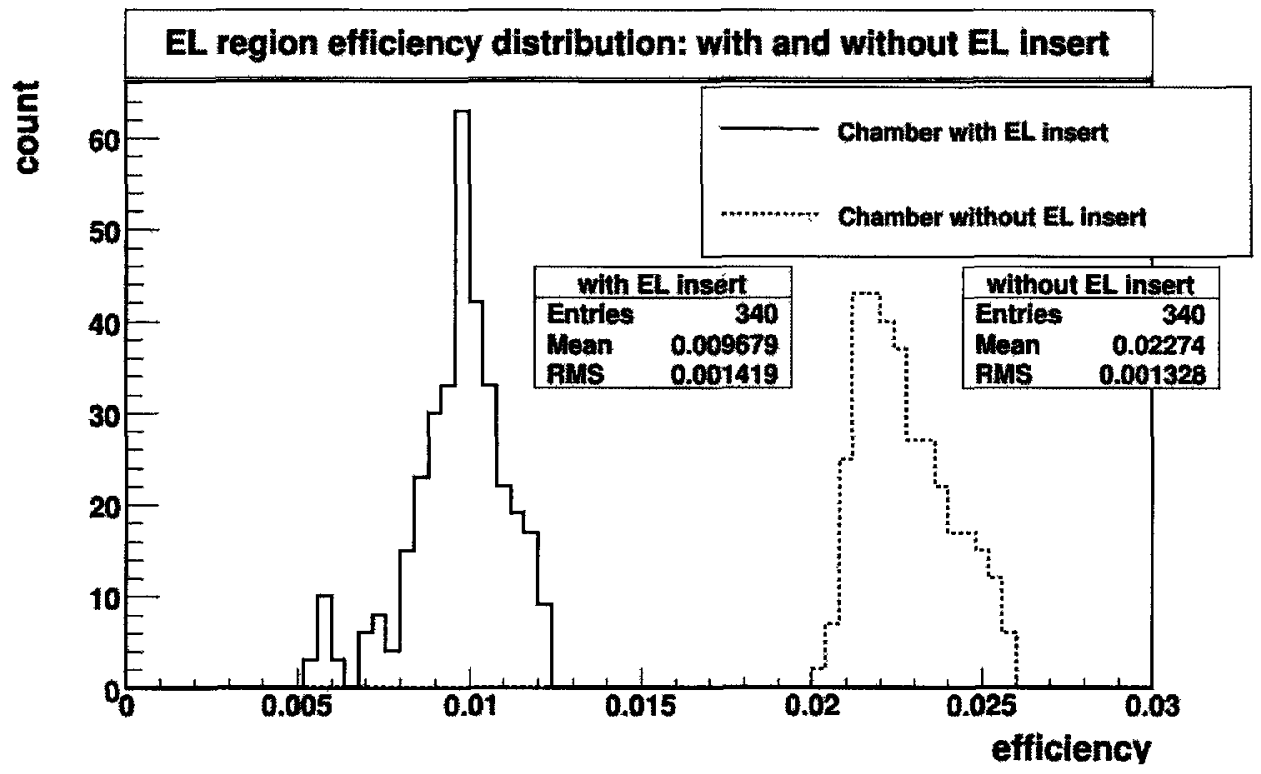

FIgURE 4.4: Light collection efficiency distributions corresponding to the EL region. Obtained with and without the EL insert. 


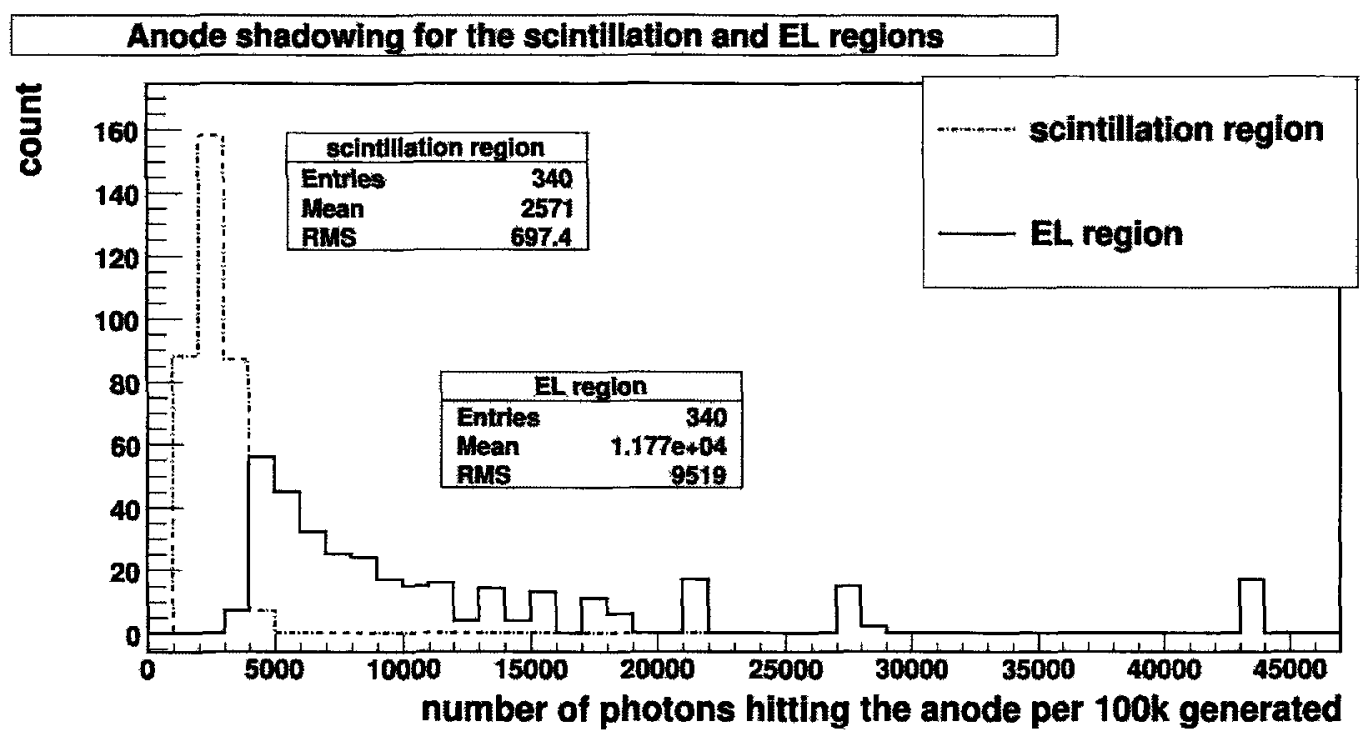

FIGURE 4.5: Anode shadowing distributions for the scintillation and EL regions.

For simulation of the EL pulse as well as to determine the efficiency for particular track angles, a point by point 3-D chamber efficiency is required. Figure 4.6 shows efficiency plots obtained as a function of $y$-position in the detector for $\mathrm{z}=-4 \mathrm{~cm}, \mathrm{z}=0 \mathrm{~cm}$, and $\mathrm{z}=4 \mathrm{~cm}$. Figure 4.7 presents the efficiencies as a function of z-position for $\mathrm{y}=0.51 \mathrm{~cm}, \mathrm{y}=5.5 \mathrm{~cm}$, and the $\mathrm{EL} /$ scintillation region boundary $y=3 \mathrm{~cm}$. Combining all of the efficiency plots forms a 2-D efficiency map for the entire y-z region, Figure 4.8. The 2-D efficiency map is then converted to a $3-\mathrm{D}$ efficiency assuming cylindrical symmetry (see Figure 4.2b), giving a point by point chamber efficiency for all regions where scintillation and EL light are produced. The chamber efficiencies range from $0.4 \%-2.2 \%$ over the entire region with the efficiency error determined from counting statistics. Assuming a 20\% PMT QE the total detection efficiency is $0.08 \%-0.44 \%$. The addition of the Teflon cap 
to the positive end of the anode introduces a small asymmetry in the efficiency response of the detector. The effect is only significant for points near the anode indicated in the efficiency plot for $y=0.51 \mathrm{~cm}$, Figure 4.7 , where the efficiency for the end with the cap $(\mathrm{z}=4 \mathrm{~cm})$ is lower than the efficiency of the end without the cap $(\mathrm{z}=-4 \mathrm{~cm})$, while for $\mathrm{y}=3 \mathrm{~cm}$ and $\mathrm{y}=5.5 \mathrm{~cm}$ there is very little asymmetry along the z-axis. Therefore the asymmetry should have little effect on scintillation signals but may have noticeable effects for the measured EL signals.
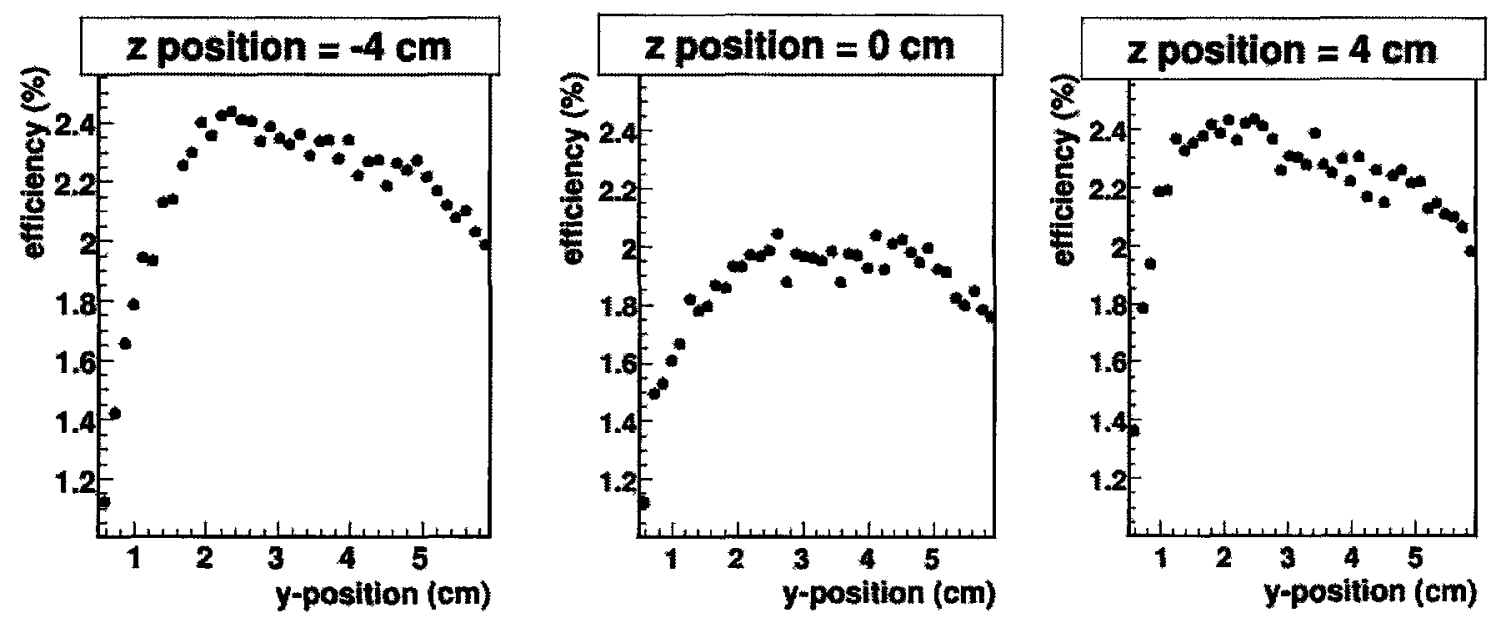

FIGURE 4.6: Sum PMT photon hit efficiency as a function of y-position for selected z-positions. 

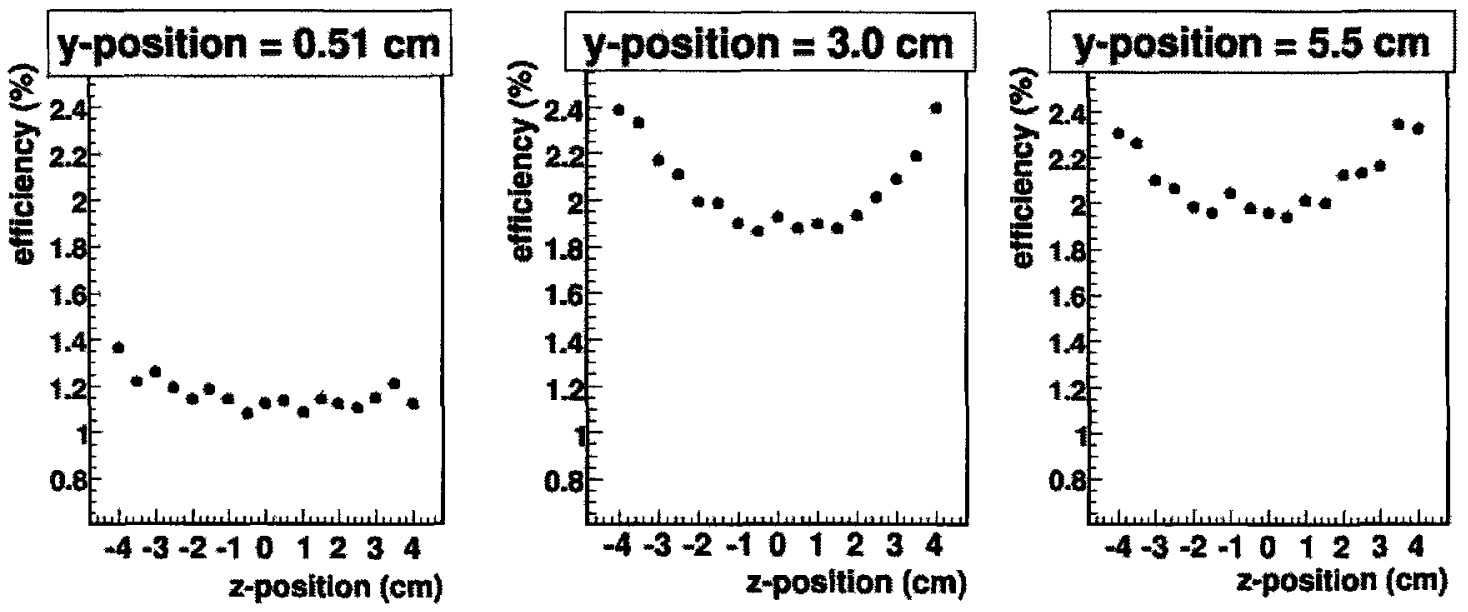

FIgURE 4.7: Sum PMT photon hit efficiency as a function of z-position for selected y-positions.

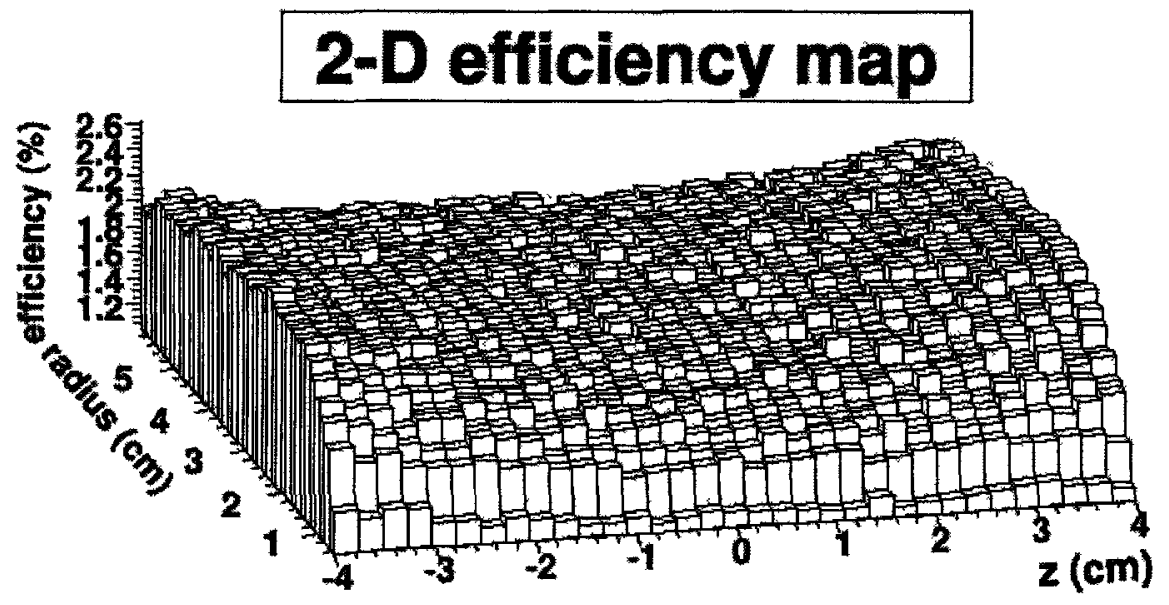

Figure 4.8: 2-D efficiency map of the $y-z$ plane for $(0.51<y<5.95) \mathrm{cm}$ and $(-4<z<4) \mathrm{cm}$. 


\subsection{Ionization \& Scintillation Pulse Simulation}

GEANT4 was also used to perform MC studies of the alpha particle interactions in xenon gas, with the primary points of interest being the ionization track orientations in the detector, their $d E / d x$ distributions, and the resultant scintillation pulses. Each event of the simulation was set to emit a $5.49 \mathrm{MeV}$ alpha particle through $2 \pi$ steradians from a primary vertex positioned at $(0.0,-6.0,0.0) \mathrm{cm}$ relative to the coordinate system defined in Figure 4.1. At every step of each event, the position, time, and energy deposited were recorded along with the process which defined the step. A typical ionization track produced by the simulation and represented by the the step points where each interaction took place is shown in Figures 4.9a,b, and c. The 3-D projection plots depict an ionization track in relation to the cylindrical EL insert for the $\mathrm{xy}, \mathrm{yz}$, and $\mathrm{zx}$ planes. The $d E / d x$ distribution obtained for the given track is shown in Figure 4.10.

The representation of the scintillation process in GEANT4 requires the input of the photon yield, along with the singlet and triplet state decay times. $W_{s}$ values of $34.3 \mathrm{eV}$ and $49.6 \mathrm{eV}$ were used to determine the scintillation yield per $\mathrm{MeV}$ of energy deposited in the gas, with 5 and $105 \mathrm{~ns}$ used for the singlet and triplet decay times respectively. The scintillation process is implemented by emitting photons isotropically through $4 \pi$ steradians, uniformly along the ionization track length, where the number of photons emitted at each point is weighted 

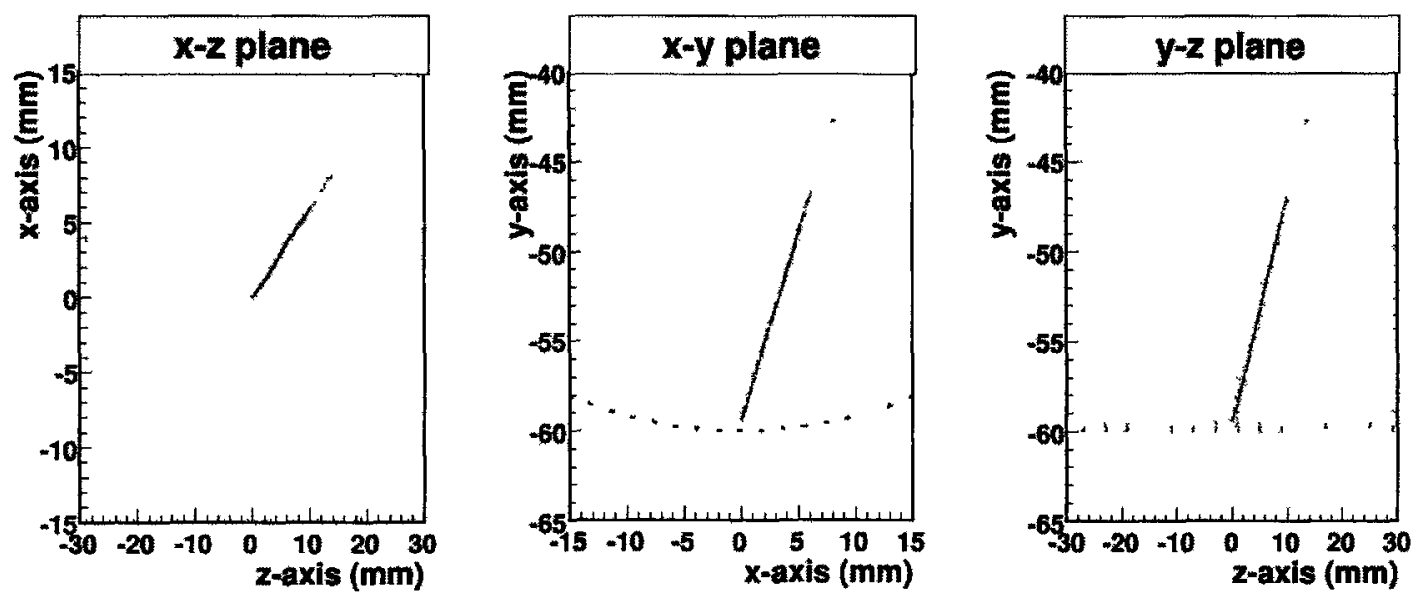

FIGURE 4.9: $\alpha$ ionization track produced in GEANT4.

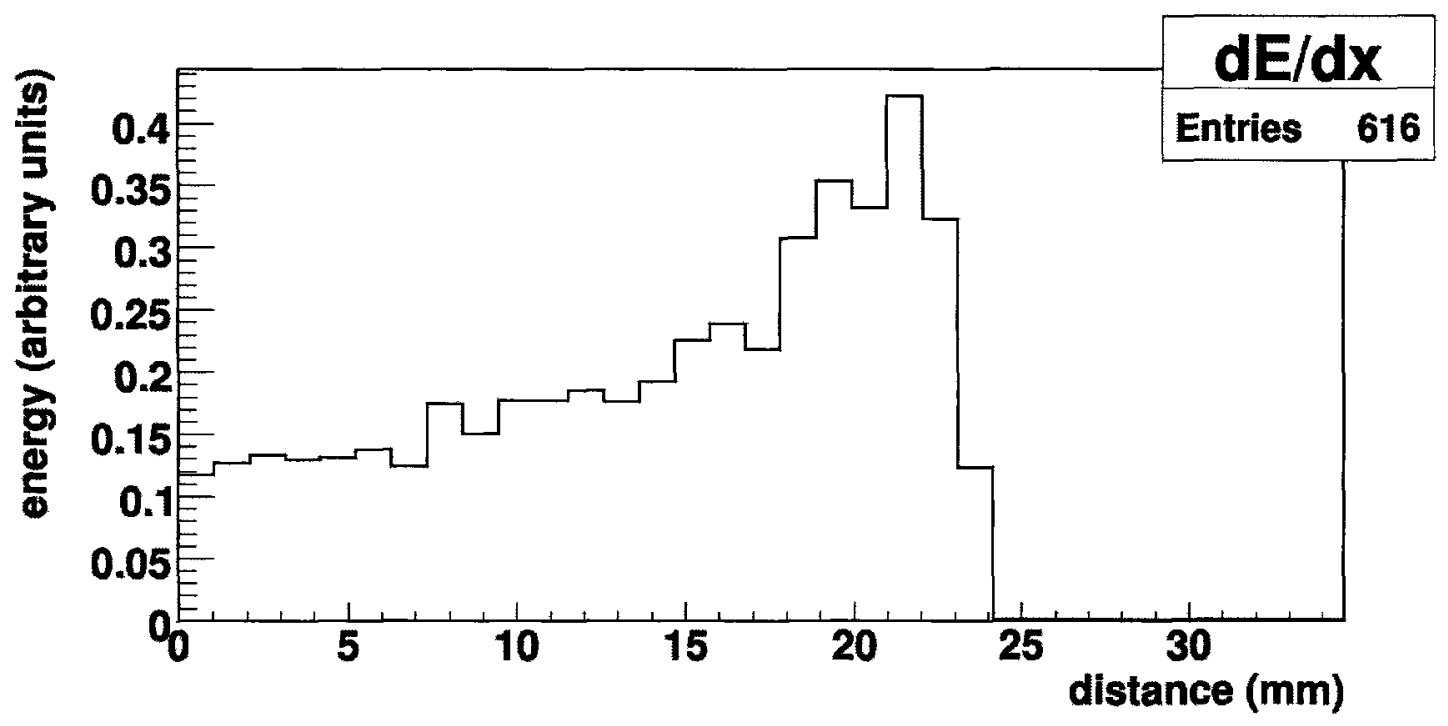

FIgURE 4.10: $\mathrm{dE} / \mathrm{dx}$ corresponding to the ionization track of Figure 4.9.

by the energy deposited, while the emission times are determined from the decay times. The simulated scintillation pulse is reconstructed using the number of photons that hit the PMT faces and their arrival times. The scintillation pulse obtained corresponding to the track shown in Figure 4.9 is given in Figure 4.11. 
The simulation was set to generate 2000 events assuming $W_{s}=34.3 \mathrm{eV}$ and a 20\% PMT QE. The distribution obtained for the sum number of photons detected by the PMTs is displayed in Figure 4.12. The number of photon hits exhibits a Gaussian distribution with a low energy tail due to range out events. The $\sigma /$ mean resolution is $\approx 5 \%$ with 573 detected photons per event expected. The simulation was also run assuming $W_{s}=49.6 \mathrm{eV}$ and a $20 \%$ PMT QE. The resulting distribution of photon hits is given in Figure 4.13, where the Gaussian fit results in 417 detected photons expected at this $\mathrm{W}$-value and an expected Gaussian $\sigma /$ mean resolution of $\approx 5 \%$.

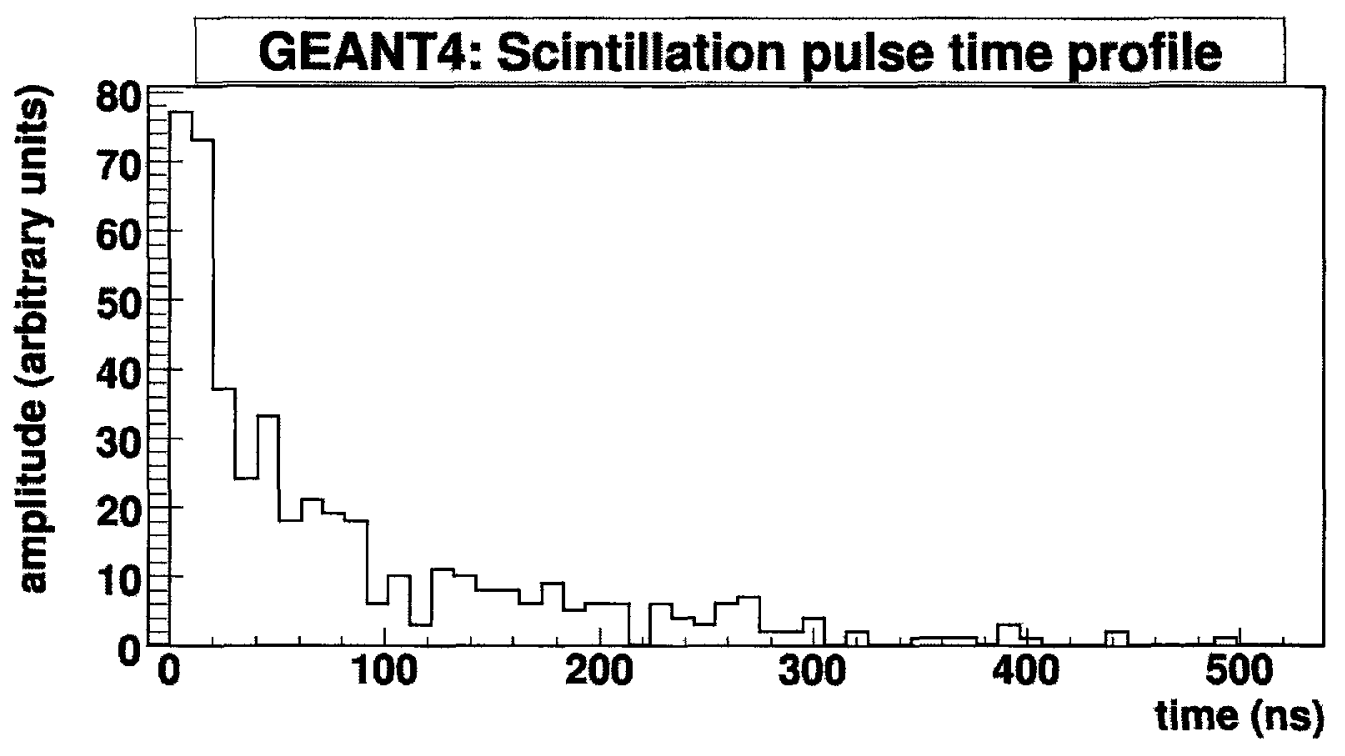

FIGURE 4.11: Scintillation pulse corresponding to the $\alpha$ event of Figure 4.9 


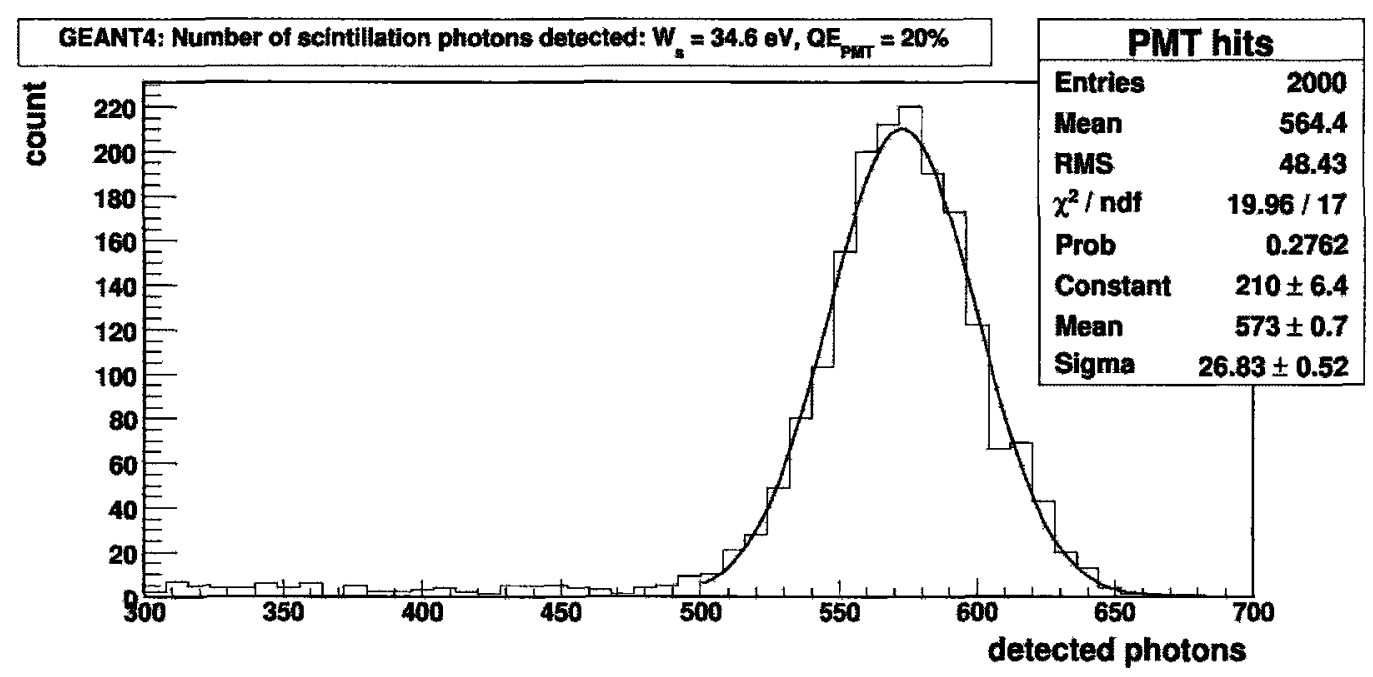

FIGURE 4.12: GEANT4 result for the number of expected photons detected by the PMTs assuming $W_{s}=34.3 \mathrm{eV}$.

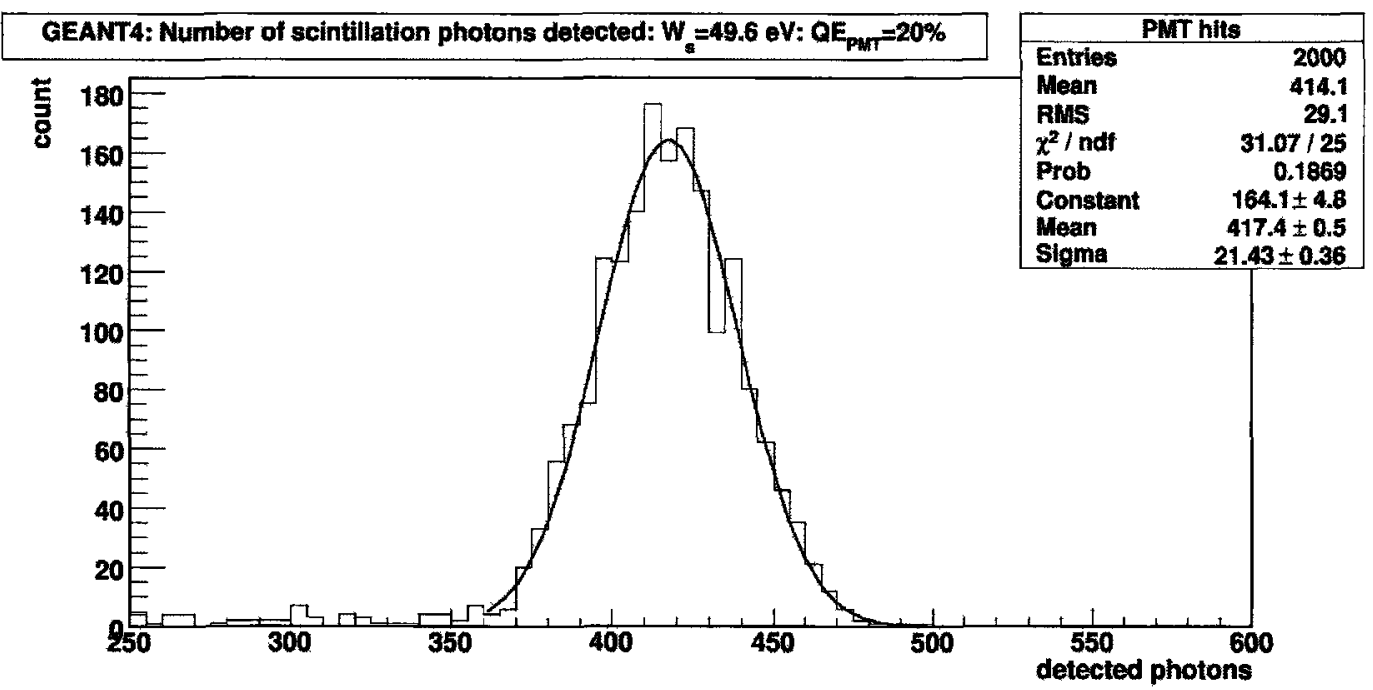

FIGURE 4.13: GEANT4 result for the number of expected photons detected by the PMTs assuming $W_{s}=49.6 \mathrm{eV}$. 


\subsection{PMT Signal Simulation}

To simulate the EL pulse and entire PMT trace corresponding to the ionization tracks generated by GEANT4 an independent ROOT program was written. The ROOT program took the position, time, and $d E / d x$ results obtained from GEANT4 and constructed EL pulses. The construction of the EL pulses was achieved by transporting the charge distribution to the anode, generating EL photons in the region defined by Equation (3.2), and according to Formula (3.4). To facilitate the transport of the charge distributions obtained from GEANT4, electron drift velocity and diffusion curves as a function of reduced electric field were obtained from the literature and input to the simulation. Each curve was digitized to 10000 points over the range of $0.1-1000$ volts $/ \mathrm{cm}$-bar. The drift velocity and longitudinal diffusion curves were obtained from Pack et al. [48].

The simulation time-step was chosen to be $10 \mathrm{~ns}$ in order to match the time binning of the raw data taken with the scope. The simulation reads in the position, time and energy deposited for each step-point of a GEANT4 ionization track. Next it calculates the electric field strength at that point from Equation (3.1). For each time-step the position and field strength are calculated and the drift velocity and diffusion are sampled from the digitized curves via interpolation to the calculated field strength. The total diffusion is calculated corresponding to drift over the distance from the step point to the anode. The charge deposited 
at the step point is spread out over 13 points following a Gaussian distribution, each one transported to the EL region boundary. Once a diffusion point reaches the EL region the charge is converted to a number of electrons and Equation (3.4) is used to determine the number of EL photons produced per time-step. The efficiency map along with the assumption of a $20 \%$ PMT QE are used to determine the efficiency at each time-step. From the efficiency the number of EL photons that reach the PMTs is determined, ie. the pulse amplitude for that time-step. This procedure is repeated for each time-step of each diffusion point until all of the charge is transported to the anode. The EL pulse is constructed from the times required to drift the entire charge distribution through the EL region and the calculated pulse amplitude for each time-step. A simulated PMT trace is obtained by combining the GEANT4 scintillation pulses with the EL pulses constructed using the corresponding ionization track. The start of the scintillation pulse is fixed at $2.5 \mu \mathrm{s}$ and this time is used as $t_{0}$ for the EL pulse construction. The PMT trace for $3 \mathrm{kV}$ obtained corresponding to the ionization track of Figure 4.9 is shown in Figure 4.14.

Raw data traces and traces obtained from the simulation were normalized to their respective integrated EL values and a comparison was made of the overall shape and trace timings for particular track orientations. The data and simulated trace timings are matched by matching the scintillation start times for each pulse, with the result for a perpendicular trace obtained at $3 \mathrm{kV}$ given in Figure 4.15. 


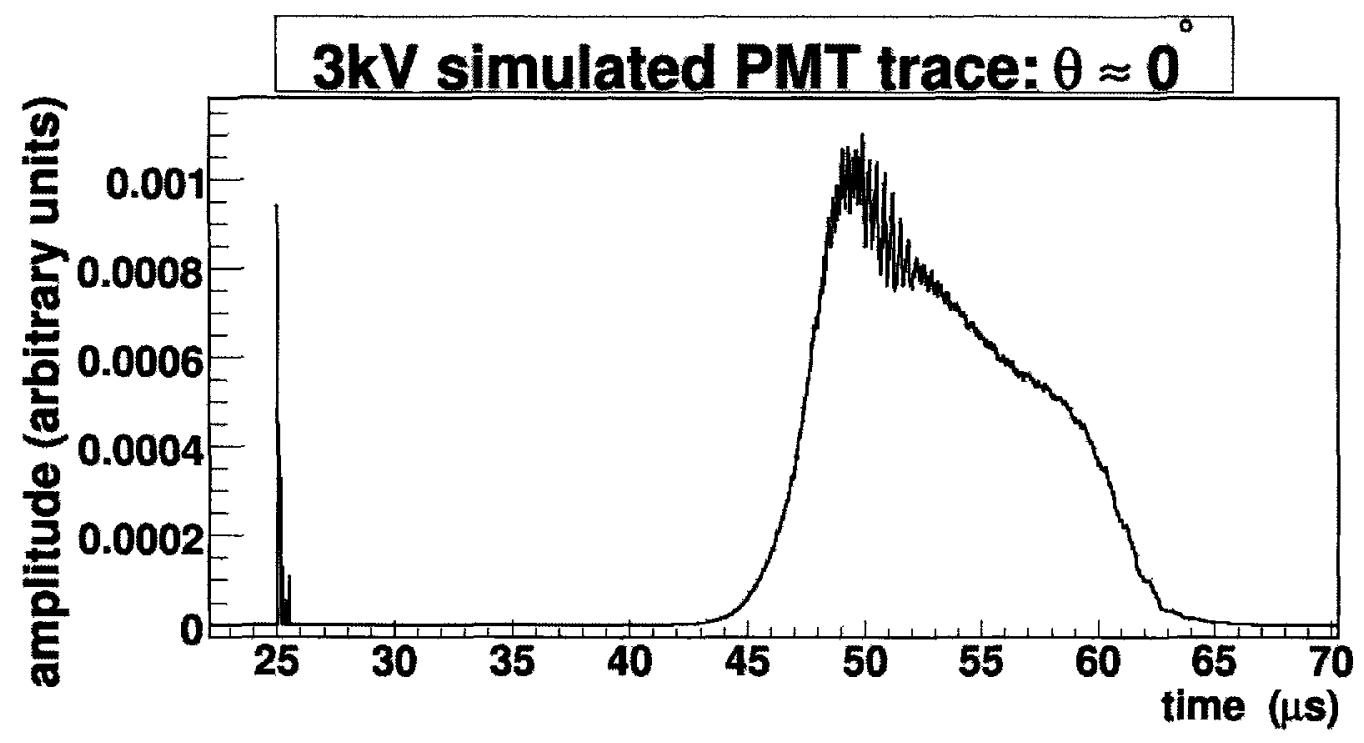

FIgURE 4.14: $3 \mathrm{kV}$ simulated PMT trace corresponding to the ionization track of Figure 4.9 .

Comparisons of the individual scintillation and EL pulses are given in Figures 4.16 and 4.17 respectively. There is a reasonable agreement between the pulse shapes and trace timings for perpendicular ionization tracks. The EL pulse comparison of parallel ionization tracks is given in Figure 4.18.

The EL pulse resulting from the simulation of parallel tracks is segmented compared to the data pulse. The segmentation of the EL pulse is caused by granularity in the method used to disperse and transport the initial charge distribution produced by GEANT4. Re-binning the traces removes the granularity and results in a improved agreement between the pulse shapes (see Figure 4.19). Figure 4.20 is a comparison of $3 \mathrm{kV}$ PMT traces for a selection of track angles. Each $d E / d x$ distribution produced by GEANT4 is made up of a collection of $600-800$ spatial points 


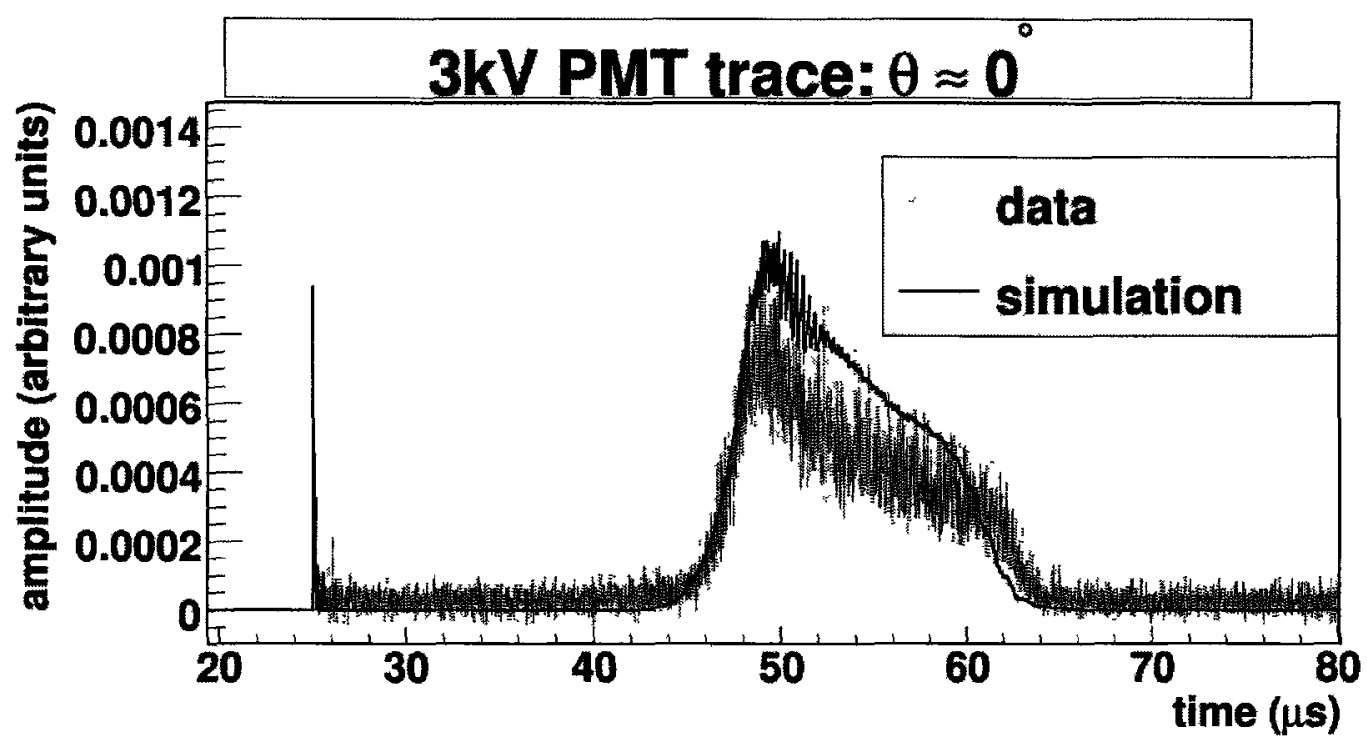

FiguRE 4.15: $3 \mathrm{kV}$ : Simulated PMT trace comparison with data: perpendicular $\alpha$ track

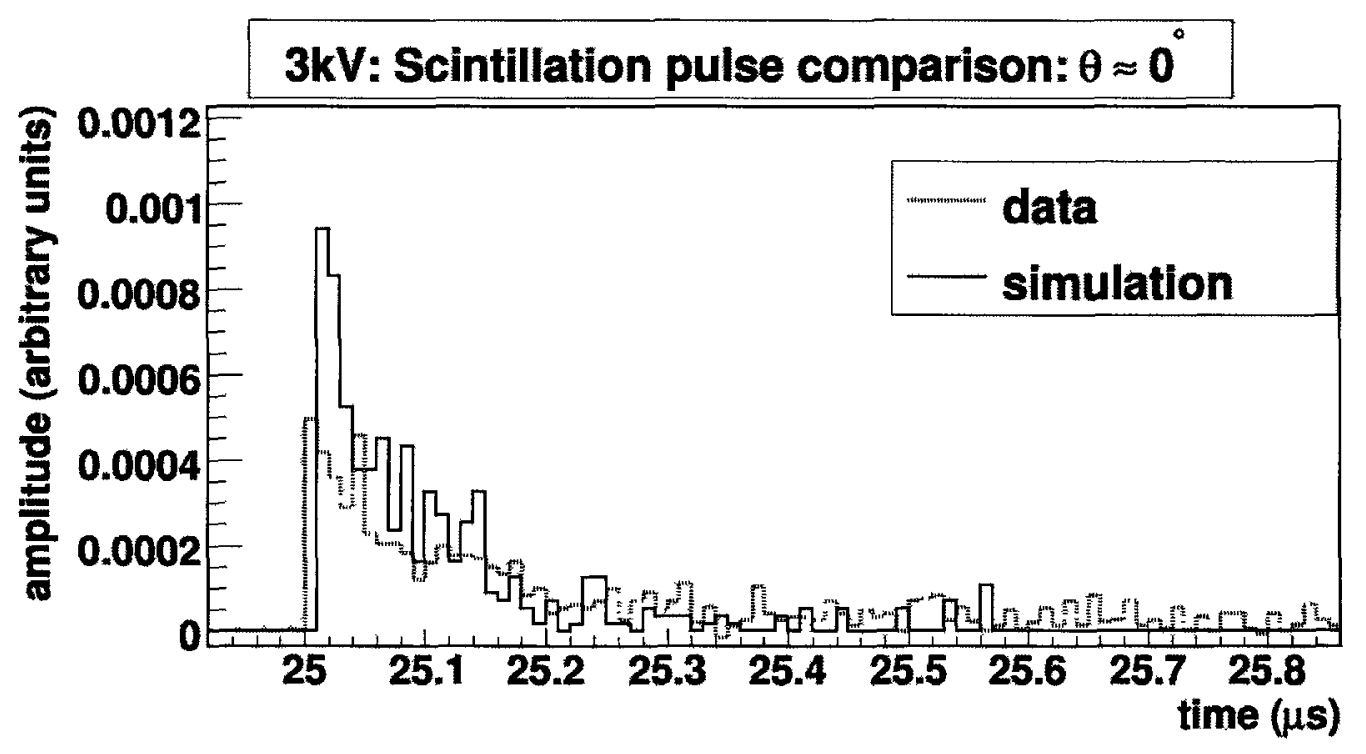

FIgURE 4.16: Comparison of real scintillation pulse to the pulse generated by GEANT4. 


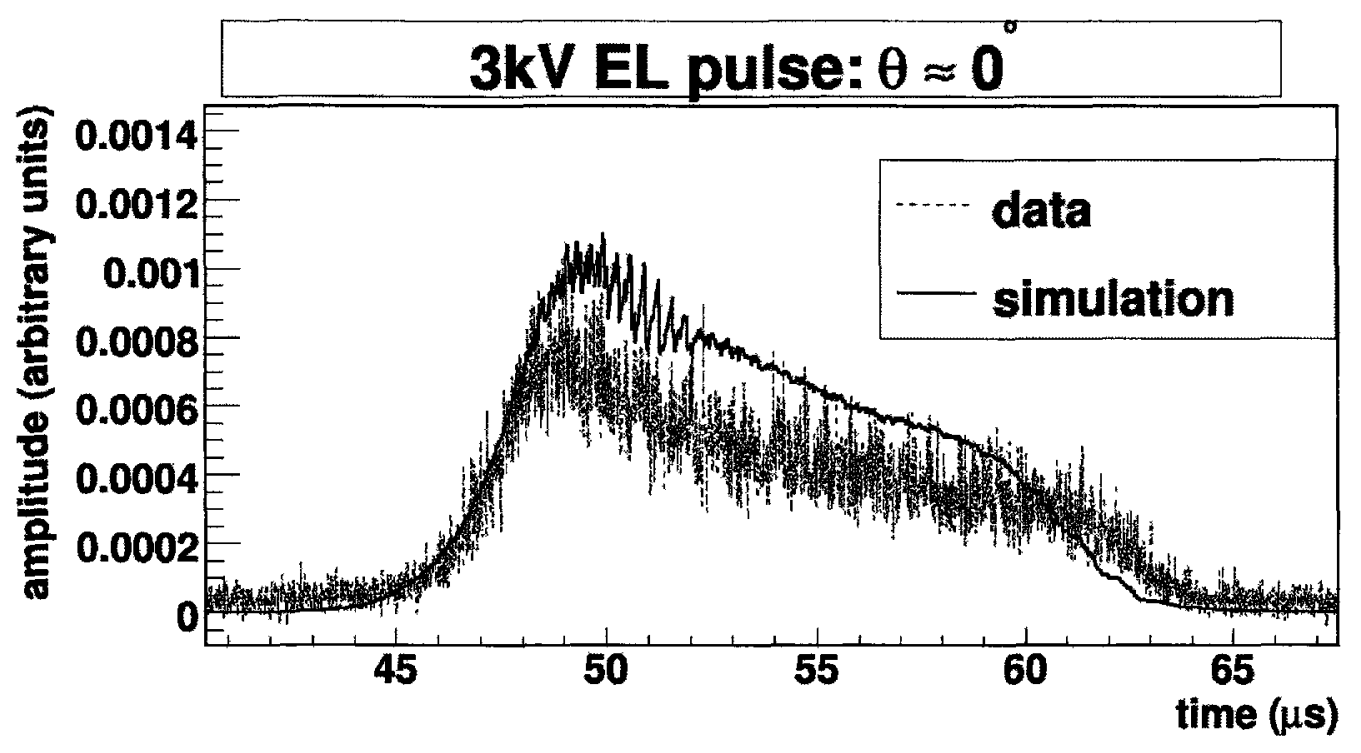

FIGURE 4.17: $3 \mathrm{kV}$ simulated EL pulse comparison with data for a perpendicular $\alpha$ track.

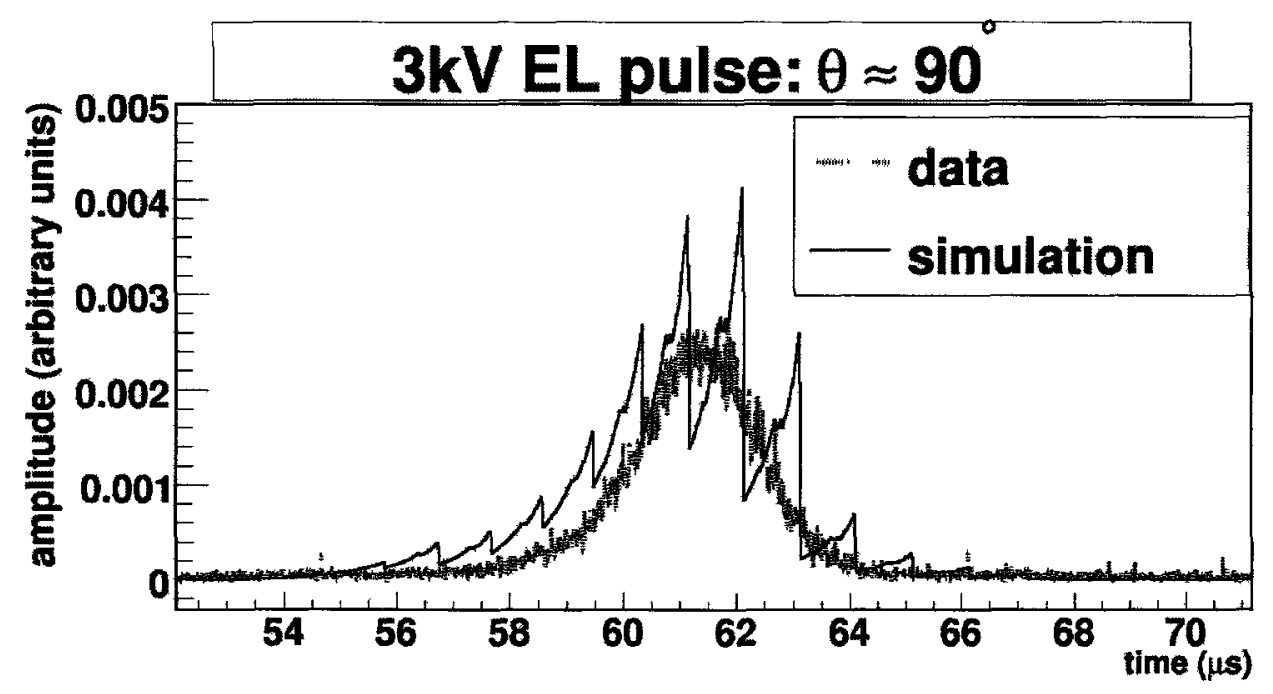

FIgURE 4.18: $3 \mathrm{kV}$ simulated EL pulse comparison with data for a parallel $\alpha$ track.

each weighted by a corresponding amount of deposited energy. The charge contained at each position however has no spatial extent, with very little radial spread 
in the points about the line of the track. Therefore the $\approx 250 \mathrm{k}$ electrons produced are spread out over the $600-800$ points, each with no spatial extent. The charge at each position is spread over 13 points following a normal distribution. The spatial position of a point of deposited charge is used as the initial position of the mean of the Gaussian distribution. The Gaussian $\sigma$ is determined from the calculated sum diffusion for drift from the initial point to the anode. Each point is then transported through the detector. The process of smearing the charge distribution over the 13 points introduces a granularity in the simulated results. Perpendicular $\alpha$ tracks are essentially parallel to the drift field and therefore granularity effects get smeared out during transport. Parallel $\alpha$ tracks are aligned perpendicular to the drift field, and the only smearing of the resulting EL distribution is introduced by distributing the charge over the Gaussian points. The resulting traces exhibit a rough granularity with 13 distinct peaks. Increasing the number of drift points would reduce the granularity, but it also dramatically increases the simulation run time. The main purpose of the MC studies was to obtain the efficiency correction factors and secondly to compare expected and measures average drift times. The PMT trace simulation acts as a check that the expected efficiencies and drift times are consistent with the data, but not as a precise analysis tool. Since precise simulation of the EL pulse shapes was not of utmost importance the number of points was left at 13 , resulting in a reasonable balance between computation time and granularity. Comparisons of the PMT traces for a selection of track angles 
obtained at a voltage of $6 \mathrm{kV}$ are given in Figure 4.21 .

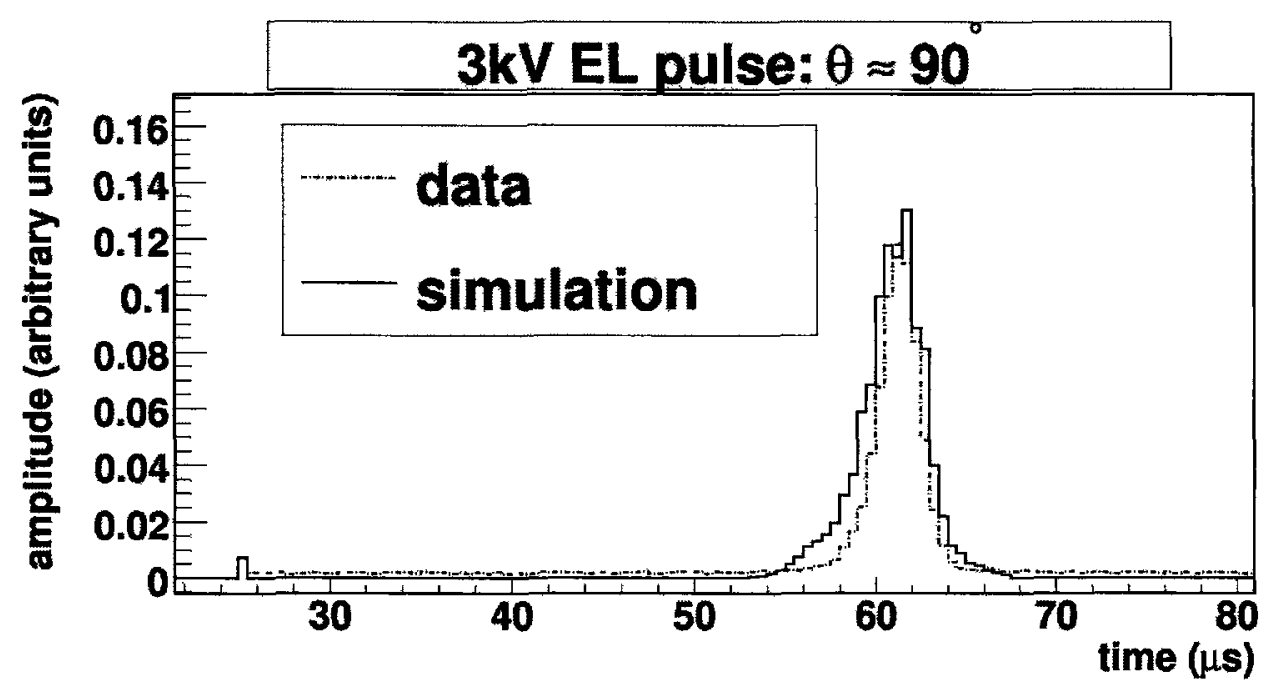

FIGURE 4.19: $3 \mathrm{kV}$ simulated EL pulse for a perpendicular $\alpha$ track: comparison with data after rebinning.

Simulated pulse amplitudes are larger then the data amplitudes. This is because the simulation uses PMT gain values obtained from the ADMCA calibration, however, this calibration method is not applicable to data acquired by the scope, resulting in the mismatch in the normalized pulse amplitudes. The spreading out the charge following a normal distribution is not completely accurate. The shape of the charge distributions will likely be non-normal due to the nonlinear drift field. The field will be higher for the leading edge of the charge cloud than the trailing edge. The differing fields will cause slightly larger drift velocities and lower longitudinal diffusion for the leading edge of the charge, resulting in a non-Gaussian distribution. If true, then the assumption of normal distribution will distort the 
resulting EL pulse shapes, with the level of discrepancy being proportional to how much the real distribution departs from normal. 


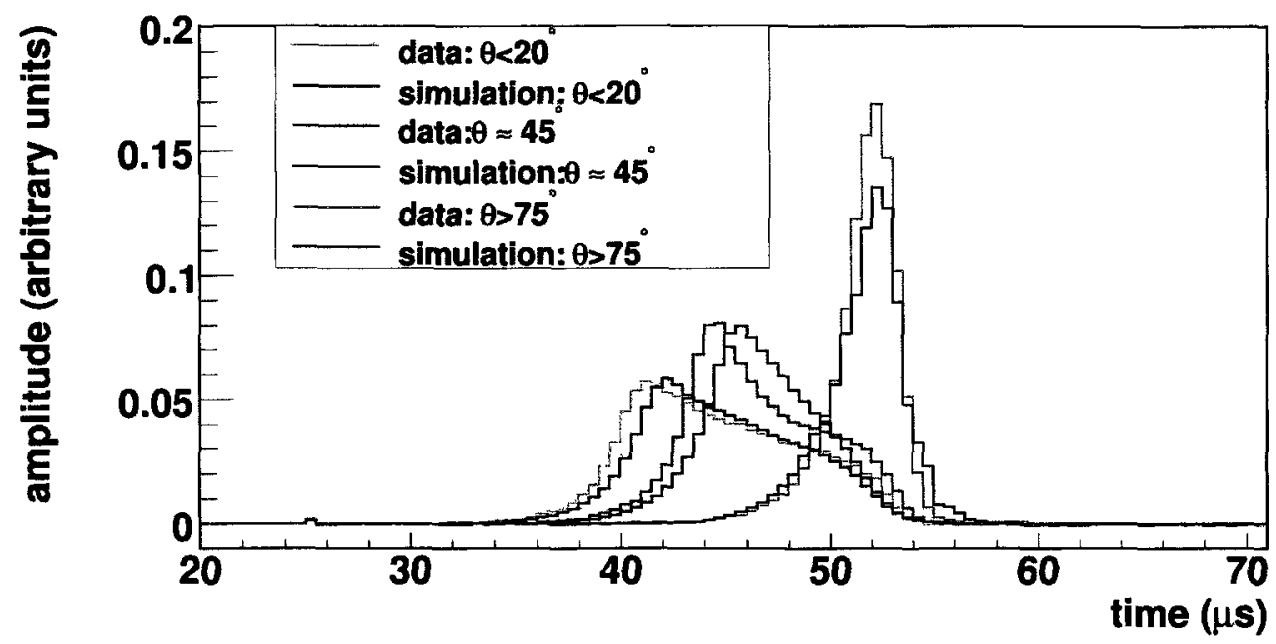

Figure 4.20: MC/data $6 \mathrm{kV} \mathrm{PMT} \mathrm{trace} \mathrm{comparison} \mathrm{for} \mathrm{a} \mathrm{selection} \mathrm{of} \alpha$ track angles.

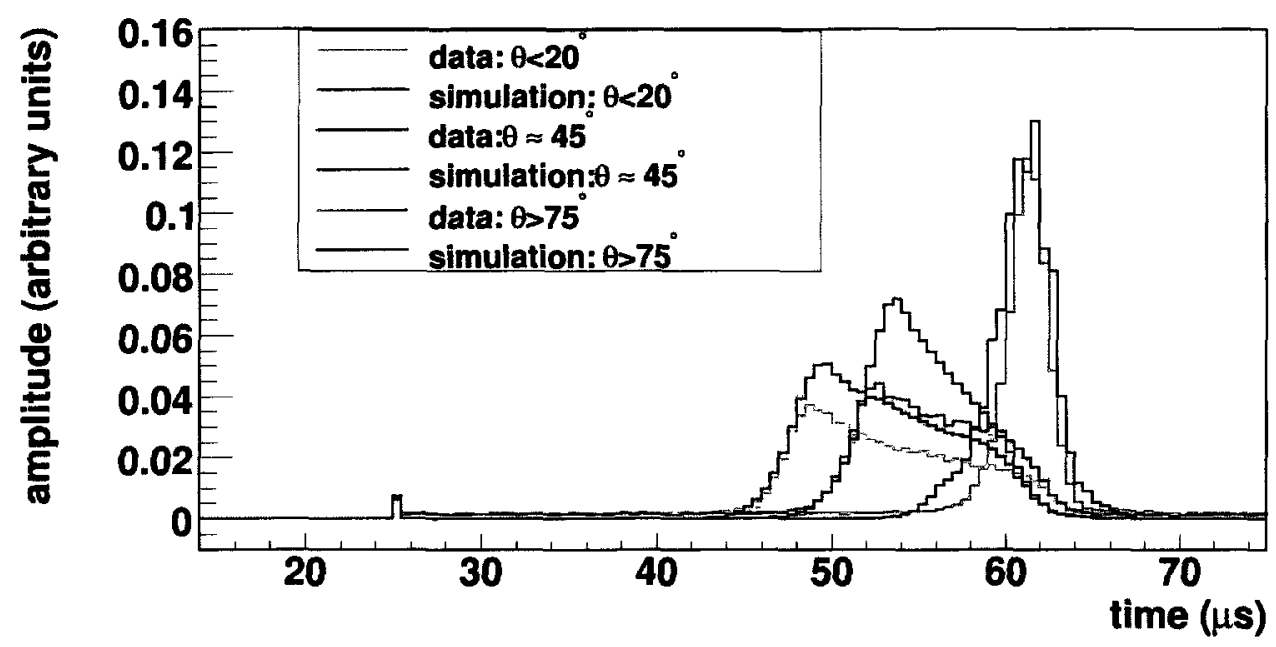

Figure 4.21: MC/data $3 \mathrm{kV}$ PMT trace comparison for a selection of $\alpha$ track angles. 


\section{Chapter 5}

\section{Data Analysis and Results}

The first step in the analysis is to convert the raw data into physical variables such as pulse amplitudes, trace baselines and timings, and integrated signal values. At this stage of processing, each raw data file is read event by event and a ROOT file made containing the variables of interest. For the PMT signals, each data trace is sequentially searched through and the scintillation and EL pulses are identified and their maximum amplitudes recorded. The scintillation start time is first approximated $\left(t_{a 0}\right)$ as being the trace time where the scintillation pulse maximum occurs. The trace baseline is found for the region $t=0 \quad n s \rightarrow t=$ $\left(t_{a 0}-250\right) \quad n s$. Then the actual scintillation pulse start time, or equivalently the event start time $\left(t_{0}\right)$, is found by starting from the scintillation pulse maximum and searching the data backwards until the first value is found that is less than 
the baseline mean plus $3 \sigma$, with the mean and $\sigma$ obtained from Gaussian fits. The time required for the onset of the EL pulse relative to the scintillation pulse is found along with the end time for the pulse by scanning through the trace from the EL maximum to its end, and finding the time-bin where the average over the 10 neighbouring bins is less then $3 \sigma$ above baseline. The integrated pulse amplitude is obtained by choosing an appropriate integration time window and summing over the pulse bins, subtracting the baseline bin by bin. For the anode signal the trace maximum and baseline are calculated, and the amplitude of the signal determined. The trace characteristics are indicated Figure 5.1 for a PMT trace obtained at $3 \mathrm{kV}$.

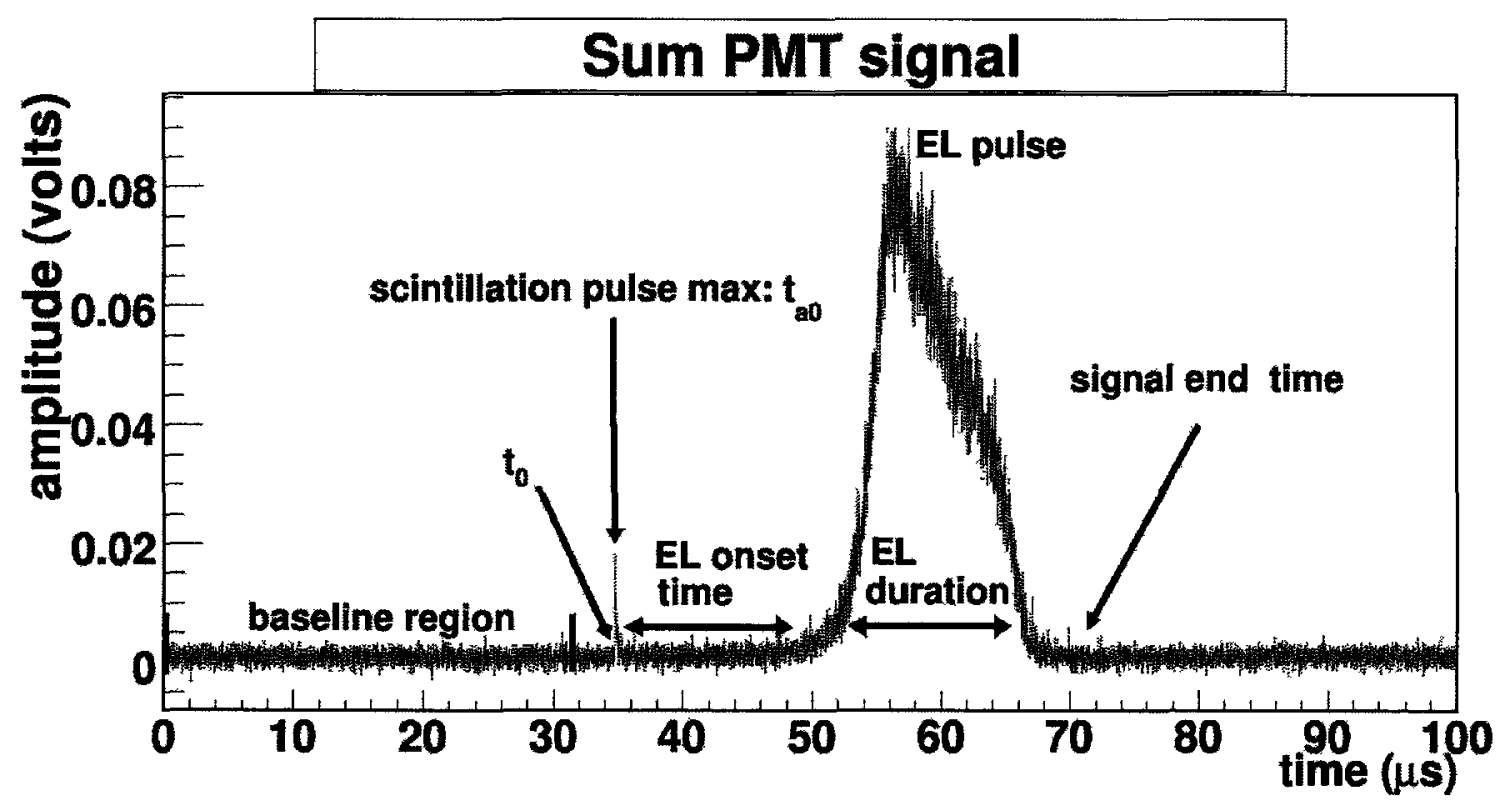

FIGURE 5.1: Raw PMT signal illustrating the trace characteristics.

During processing there are a number of quality checks of the data were 
made and any event that does not meet the criteria is removed from the analysis. The three main reasons for cutting an event are:

- Signal pileup: Two alpha particles that are emitted within a single event time window, causing two scintillation and EL pulses on a single data trace. The anode signal amplitude is also much higher due to the drift and collection of the extra charge. The anode signal was used to cut these events by requiring that the amplitude of the signal after baseline subtraction was not above a tolerance set to $1.3 \times$ the expected maximum amplitude.

- Event windowing: Not all events acquired end up centered in the data acquisition window. Scintillation and EL pulses may end up cut off at the trace boundaries or be completely missed. These events are cut by requiring that the obtained EL and scintillation pulse maxima are located within defined time windows and any events that do not have both pulses within the defined times are rejected.

- Null events: The trigger was set on the scintillation and anode signals depending on the applied drift field. Sometimes the trigger would miss-fire on noise and the acquired trace would not contain any signal. These events are removed by requiring that value of the trace maximum is larger then a cutoff defined by the noise on the signal. 
The number of events of a particular run that does not meet the criteria is on the order of $5 \%$ for all the operating voltages. The full details and final results of the analysis will now be discussed.

\subsection{PMT Baselines}

The resulting scintillation and EL values are dependent on the PMT trace baselines due to the bin by bin subtraction in integrating the over the pulses. Each trace consists of $10 \mathrm{k}$ data points taken over $100 \mu s$, resulting in $10 \mathrm{~ns}$ binning. To characterize the trace baseline, the baseline is obtained for the region $t=0 \rightarrow$ $\left(t_{0}-3\right) \mu s$ by a number of methods. First the baseline and error are determined from the average of the data over the region. Second the baseline and error are obtained from fitting the region with a constant function. A third baseline is obtained for the region by recalculating the average baseline but excluding any data points that lie outside of $3 \sigma$ of the mean obtained from a Gaussian fit to the original baseline distribution. Finally, a fourth baseline is found from a fit to the end of the trace over the region $t=(95 \rightarrow 100) \mu s$. Figure 5.2 is a PMT trace obtained at $3 \mathrm{kV}$ showing the results from fitting the beginning and end of a PMT trace with a constant function. Figure 5.3 gives the original data distribution over the baseline region and the distribution obtained excluding events which lie outside $3 \sigma$ corresponding to the original distribution. The distribution of the 
baseline values obtained for the selected voltages of 2,4 , and $6 \mathrm{kV}$ are shown in Figure 5.4.
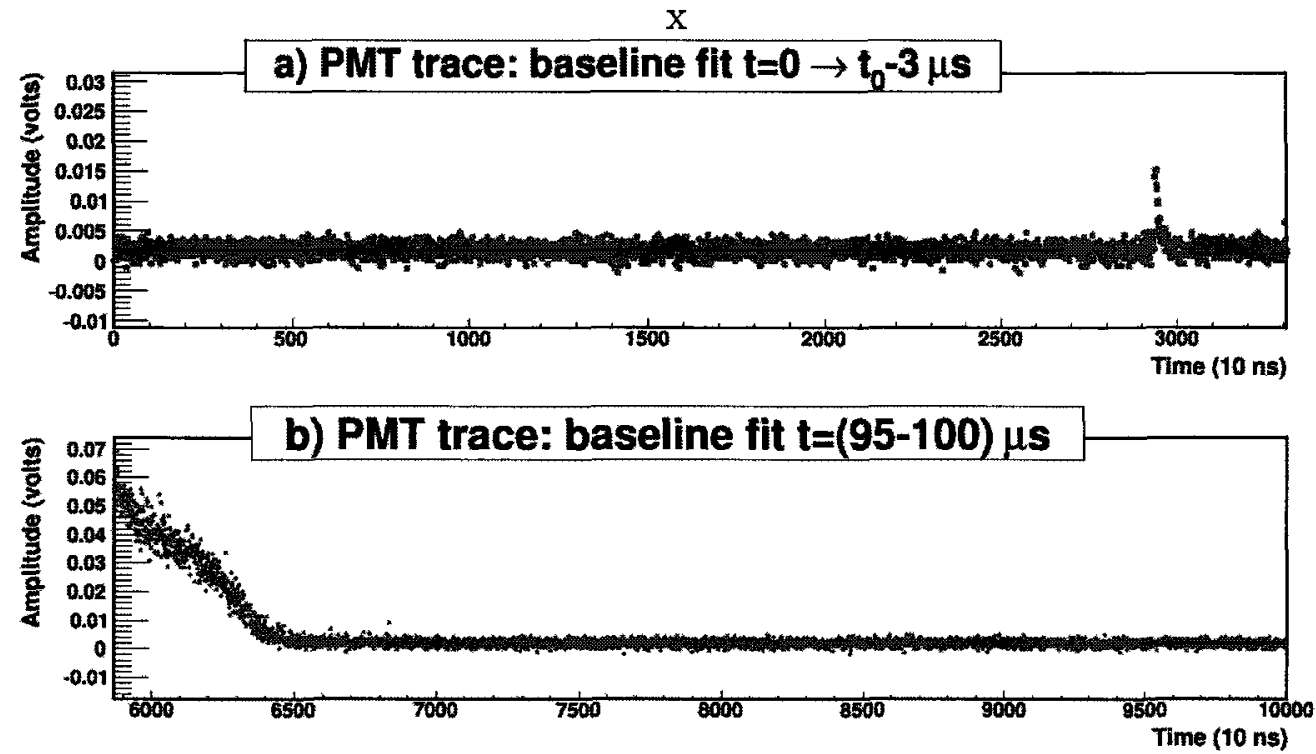

FIgURE 5.2: PMT baselines obtained from fit: 1) baseline fit over region $t=$ $0 \rightarrow t=t_{0}-3 \mu \mathrm{s} .2$ ) baseline fit over region $t=95 \mu \mathrm{s} \rightarrow t=100 \mu \mathrm{s}$

The baseline distributions obtained from the fit to the beginning and end of the trace are in excellent agreement. This indicates the trace time window used was sufficiently long to ensure that all the light was collected for each event and the PMT baseline returned to its initial value after the EL pulse. The two distributions corresponding to the mean baseline and the mean baseline excluding points residing outside of $3 \sigma$ are also in excellent agreement, where $\sigma$ is obtained from a Gaussian fit to the original baseline distribution. This implies the region of the trace used to find the baseline values does not contain signal. The baseline values obtained from the fit and distribution means are also in excellent agreement. 

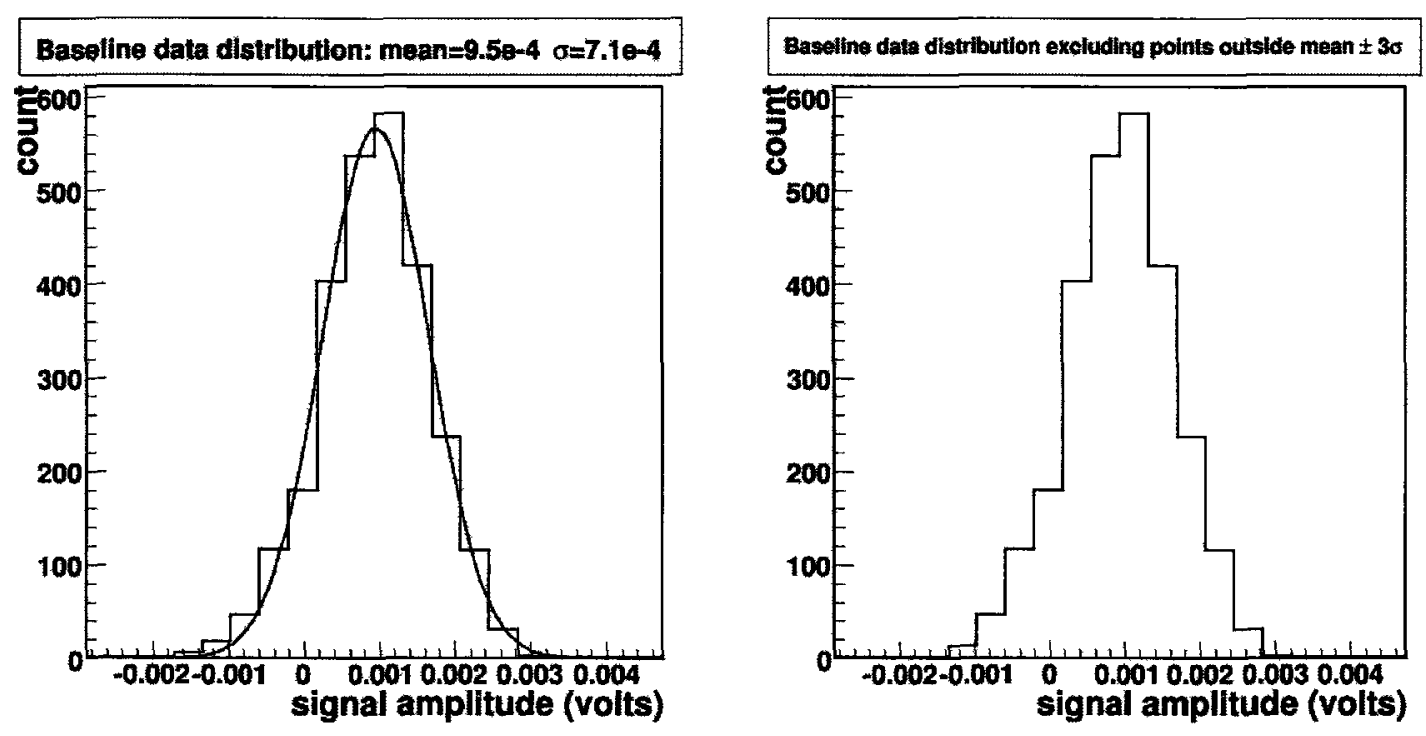

FIGURE 5.3: PMT average baselines over the region $\left.t=0 \rightarrow t=t_{0}-3 \mu s: 1\right)$ raw distribution of data. 2) distribution of data excluding points outside $3 \sigma$
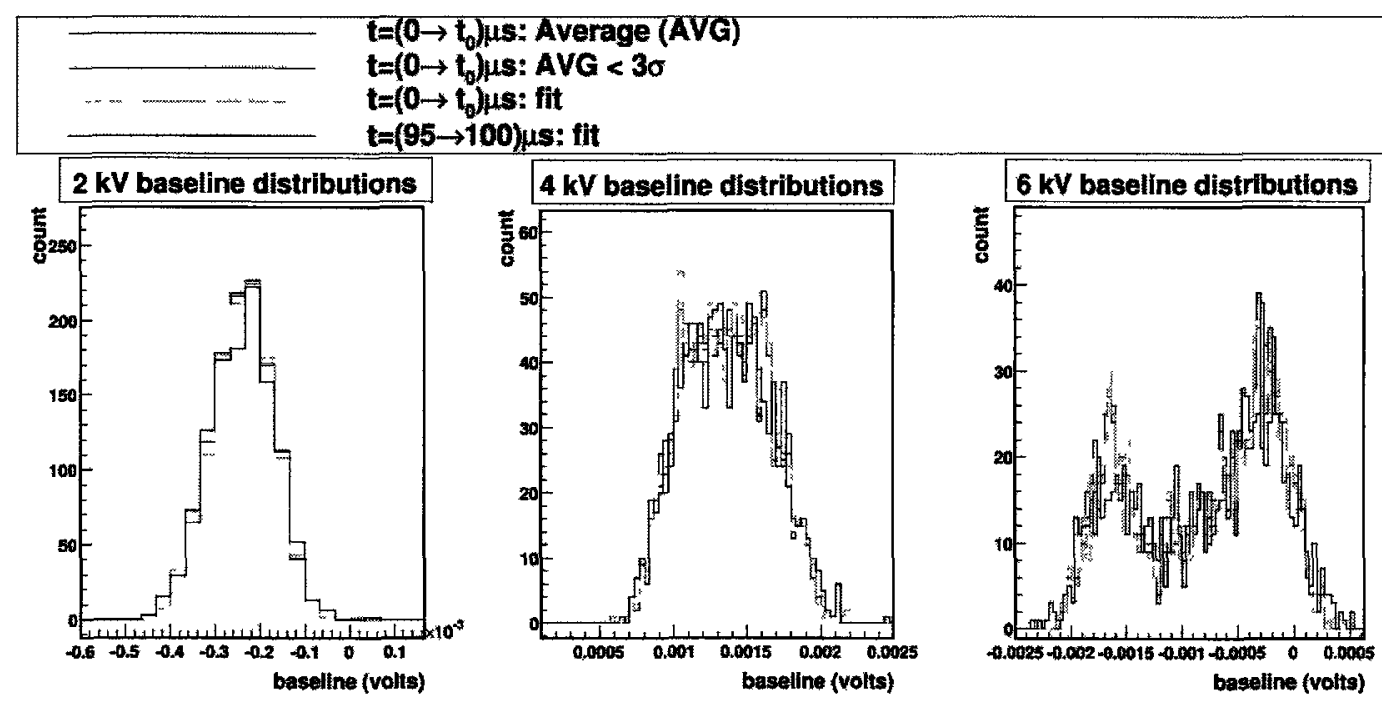

FIGURE 5.4: Baseline distributions obtained for voltages of 2, 4, and $6 \mathrm{kV}$.

Figures 5.5 and 5.6 give plots of the mean and RMS of the obtained baseline distributions versus high-voltage. All values follow the same trend with increasing 
voltage, with the mean and RMS in excellent agreement. Therefore the use of either method for calculating the trace baseline should result in similar integrated values.

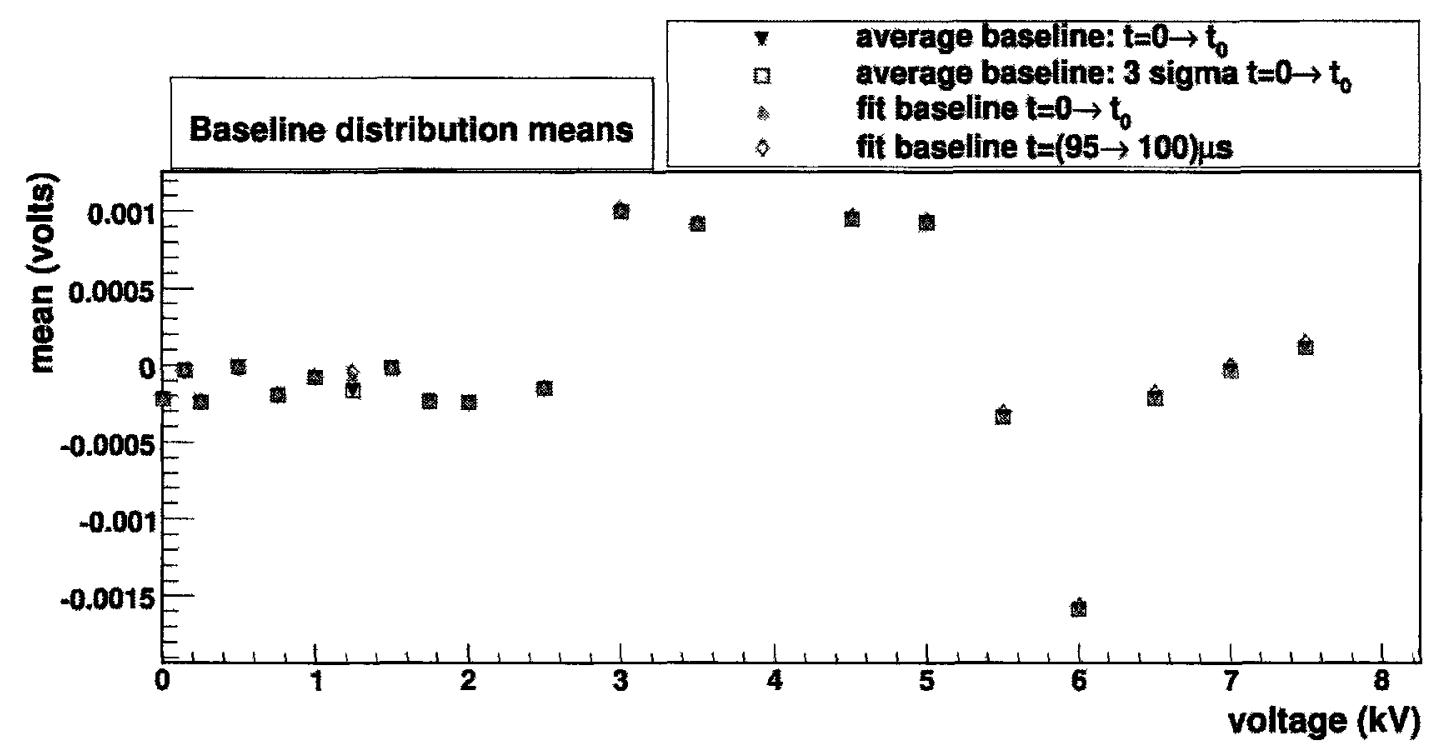

FIGURE 5.5: Baseline mean values vs. voltage.

Any difference between the mean and fit baseline values will result in a difference in the integrated signal values. The difference between the mean and error of the distributions resulting from the two methods can be considered as a source of systematic error introduced in the measurements due to the baseline subtraction. Results will be presented for the baseline obtained from the distribution mean and any discrepancy between the parameters obtained using the fit baseline will be used in assigning a rough systematic error. 


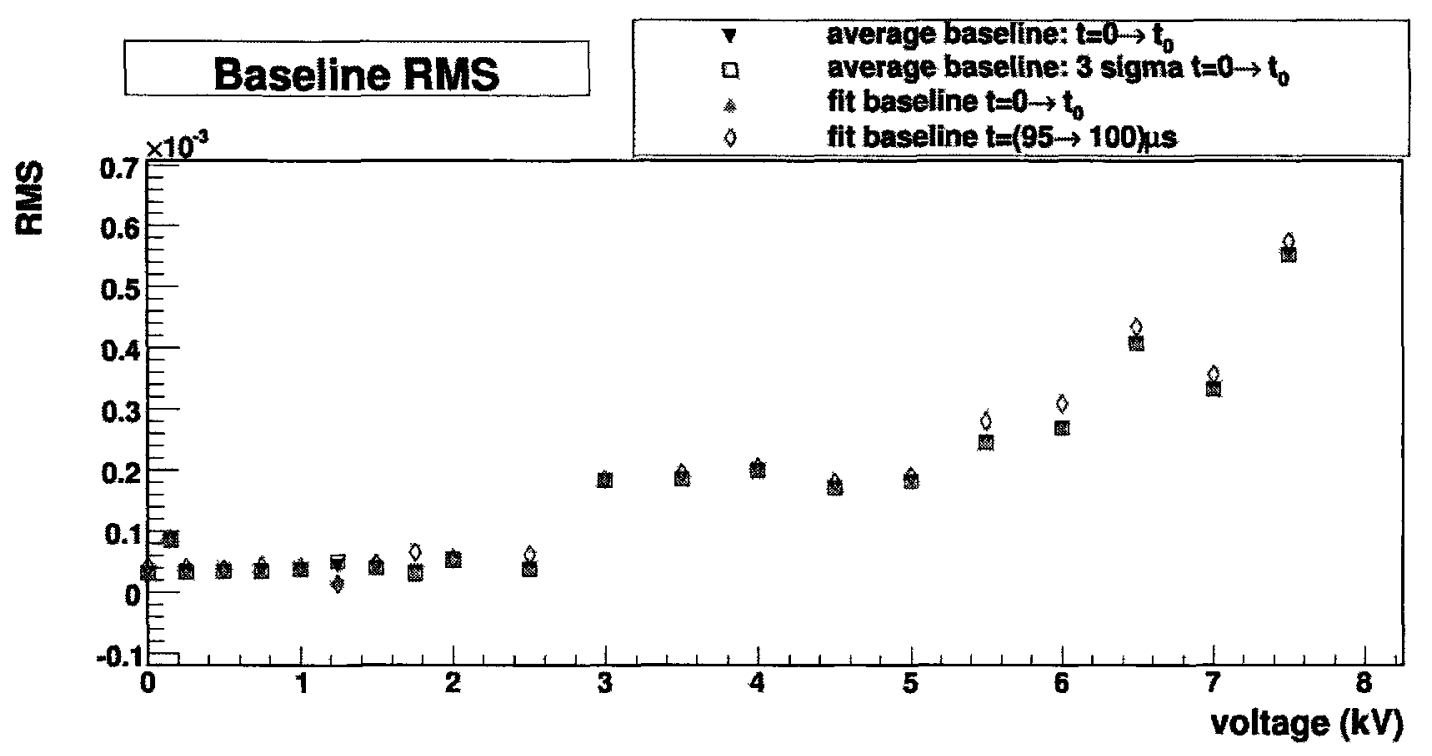

FiguRE 5.6: Baseline RMS vs. voltage.

\subsection{Track Angle Cuts and Efficiency}

In order to characterize the orientation of each ionization track, a number of trace timings were determined relative to the event start time $\left(t_{0}=\right.$ scintillation pulse start time). The time required for the charge to drift from its initial position to the EL region was determined from the PMT traces by finding the onset time of the EL pulse from an exponential fit to the pulse's leading edge. The result of fitting the leading edge of the EL pulse is presented in Figure 5.7, resulting in an onset time of $\approx 15.3 \mu s$.

The fit parameters are used for determining the EL onset time relative to $t_{0}$ by determining where the function exceeds $3 \sigma$ above baseline, indicated by the 


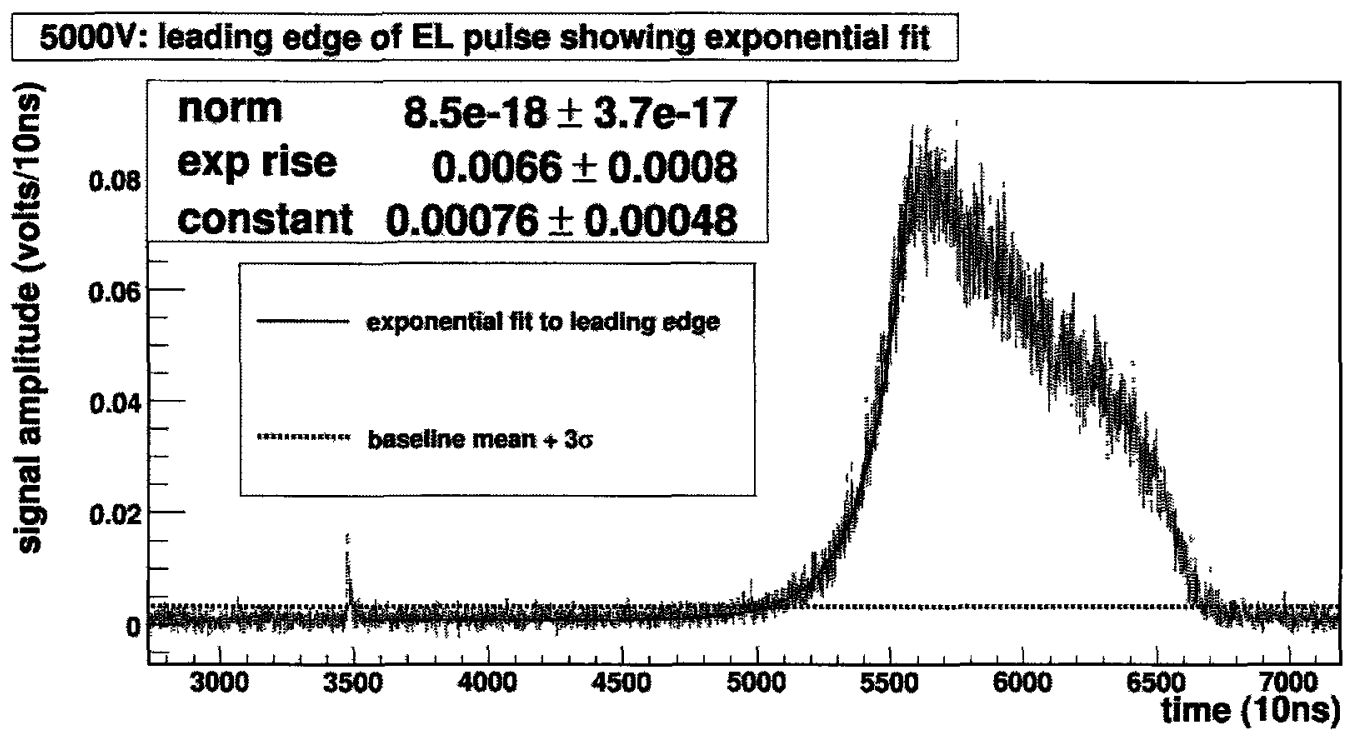

FIGURE 5.7: Exponential fit to the leading edge of a perpendicular $\alpha$ track.

blue line in Figure 5.7, with the Gaussian $\sigma$ obtained from Figure 5.3. Parallel (perpendicular) tracks correspond to the shortest (longest) times of the resulting time distribution. The PMT signal end time was found from the trace region after the EL pulse, by determining the time bin where the average found over the 5 bins on either side ( $50 \mathrm{ns)} \mathrm{had} \mathrm{dropped} \mathrm{back} \mathrm{to} \mathrm{the} \mathrm{baseline} \mathrm{value.} \mathrm{For} \mathrm{each}$ trace the signal end time, the EL pulse onset and duration times were measured. Ionization tracks corresponding to $\theta \approx 90^{\circ}$ result in the longest $\mathrm{EL}$ onset times and have the shortest EL duration, while tracks corresponding to $\theta \approx 0^{\circ}$ result in the shortest EL onset times and have the longest EL duration. The signal endtime is approximately constant for all track angles. The end-time is defined by the time required for the last electron to be collected on the anode and ionization is produced along the entire length of a track, therefore the total drift distance 
for the charge produced at the start of a track is roughly fixed at $6 \mathrm{~cm}$. By using the drift times along with known drift distances the average drift velocity over the various drift regions are found. The methods of the trace simulation discussed in Chapter 4 are used to predict the drift times and velocities for the regions and compare these to the measured values. Plots of the EL onset time and total signal duration are given in Figure 5.8 as a function of applied voltage, with the corresponding average drift velocities given in Figure 5.9. The ratios of expected to measured drift velocities are presented in Figure 5.10.

The error is assigned based on estimates of the accuracy of EL onset and signal end-time measurements. A $2 \%$ error on the drift times found from the data, and a $0.5 \%$ error on the drift times obtained from the simulation were assumed and propagated through the calculations to obtain the given errors. The final error on the ratio of average drift velocities is on the order of $10 \%$. Since the drift velocity of electrons is highly dependent on gas purity at the level of ppm, drift velocity measurements can and were used as a gas contamination check. Gas contamination was found in earlier runs by comparing the drift velocities, where drift velocity ratios ranged from 1-2 over the voltage range, indicating something was wrong with the gas. The chamber was evacuated and leak tested with a helium leak check detector, where small diffusive leaks were found and fixed. The chamber was then re-filled with new xenon. The level of agreement in the current ratio measurements agree with 1 within the given errors. 

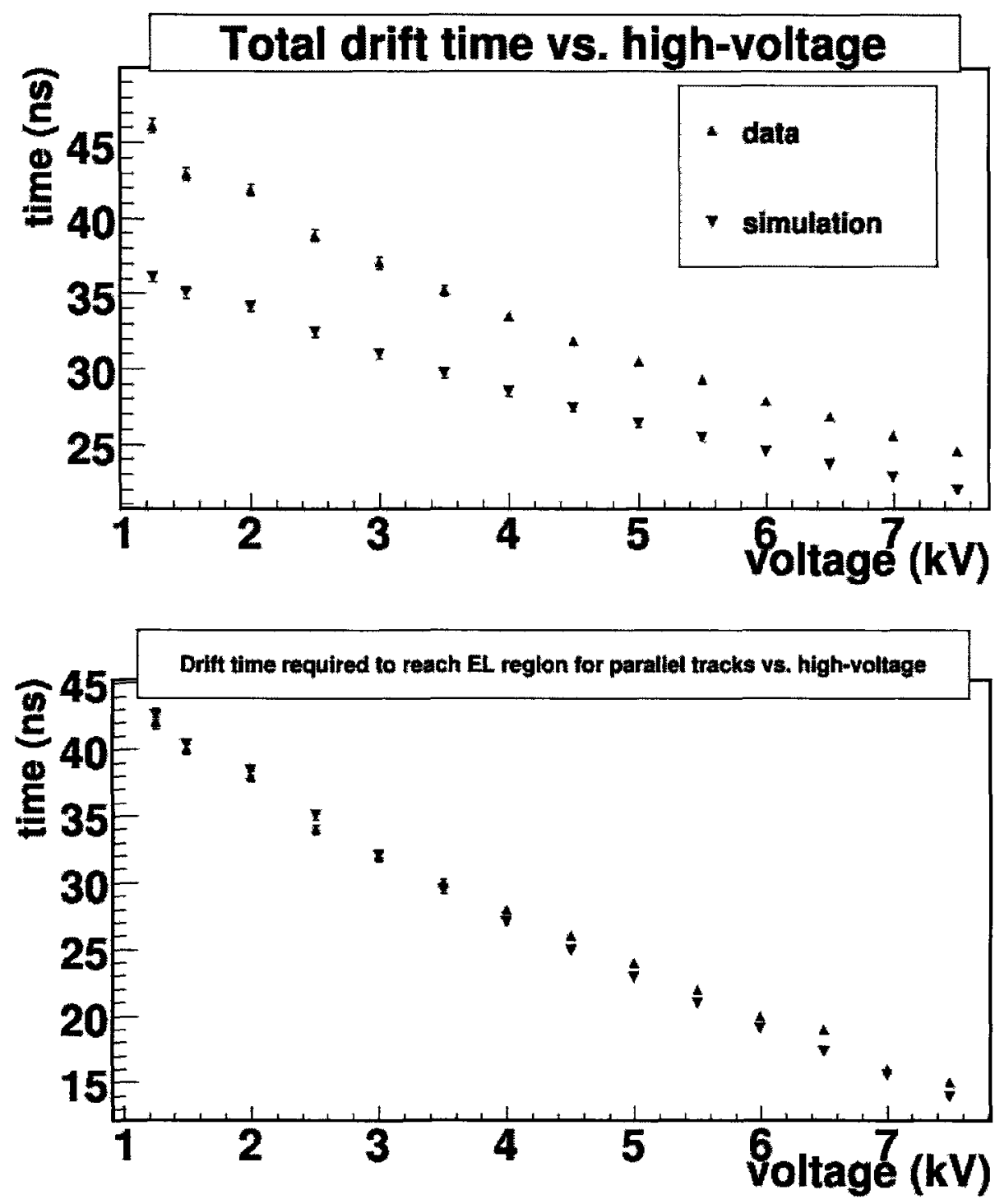

FiguRE 5.8: Signal and EL onset drift times: measured values and simulated results

The values obtained for the integrated EL signal and anode amplitudes are dependent on the ionization track orientation and therefore will affect the energy resolution. There are a number of geometrical factors which influence signal amplitudes and shapes. Anode amplitudes are larger for parallel tracks due 

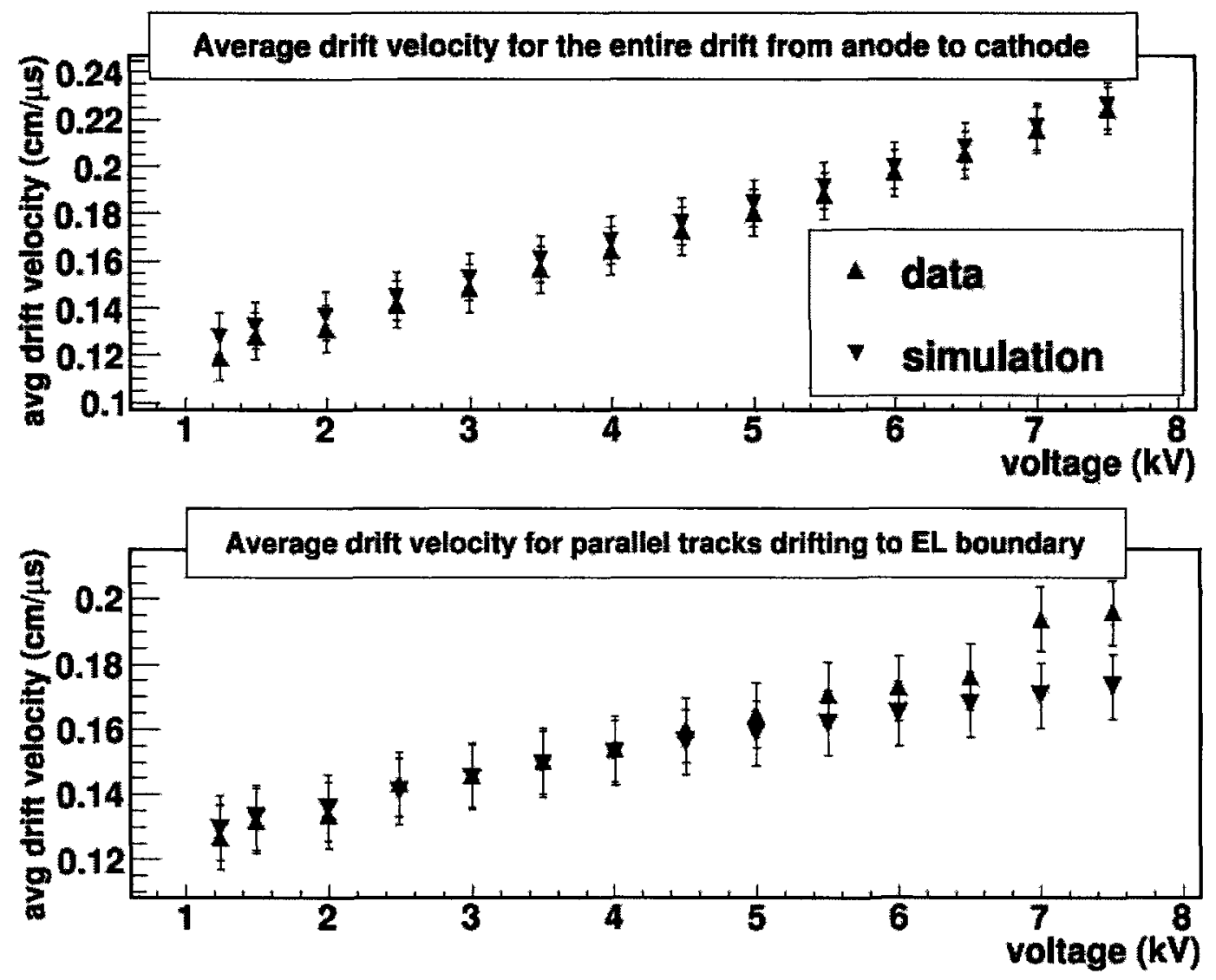

FIGURE 5.9: Average drift velocities for drift to the EL region and for total charge collection: measured values $\&$ simulated results

to the charge drifting over longer distances and inducing bigger currents on the anode. The integrated EL signal on the other hand follows the opposite trend, with larger integrated amplitudes corresponding to perpendicular tracks. Alpha particles emitted parallel to the source plane lose more energy then perpendicular tracks since they traverse more source material while leaving the surface, producing less ionization. The majority of the charge produced has to drift over a longer 

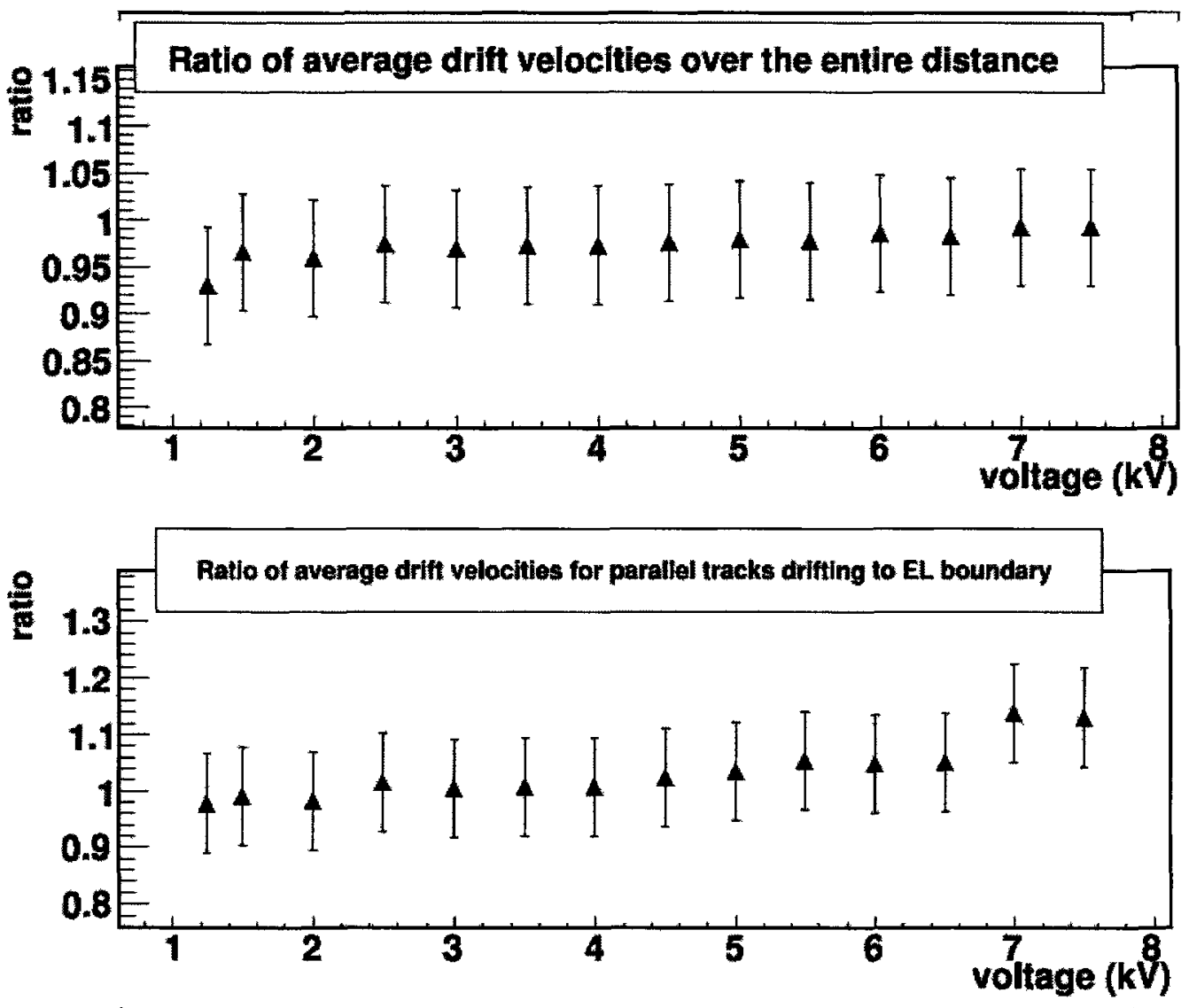

FIGURE 5.10: Ratio of the measured to simulated average drift velocities.

distance and a larger fraction can be lost due to capture by electronegative impurities. A plot of the EL signal versus the $\mathrm{EL}$ onset time obtained at $6 \mathrm{kV}$ is given in Figure 5.11a, with the corresponding onset time distribution given in Figure 5.11b. The slope of the EL value with onset time, Figure 5.11a, is due to the loss of energy from electron capture and source plane effects. The low energy tail of the distribution for long drift times is due to range out events that deposit energy into the cathode. The best EL energy resolution will be achieved for small angle 
ionization tracks since the amplitude will be the largest and energy loss will be minimal. The EL onset time distribution is a function of the angle of $\alpha$ emission, with the shortest times corresponding to small $\theta \mathrm{s}$ and the largest times to large $\theta$. In order to determine the angular dependence of the EL value and subsequent measurements, event cuts were applied by selecting time ranges based on slices of the EL onset distribution.

The onset distribution for each voltage is cut into 30 time sections starting with the shortest onset times, with each section containing between 1000 and 1200 events. The resulting time cuts are depicted in Figure 5.11b, where the red and blue markers represent the start and end points for the cuts respectively. By using the measured times and applying the electron transport simulation, the drift distances and angular range corresponding to a particular cut are found. Using the angular distribution and applying the chamber efficiency map, the average efficiency of the scintillation and EL regions for each time cut and voltage is determined. The procedure is applied to each voltage resulting in an average efficiency of the light collection for each voltage and $\theta$ range. Figure $5.11 \mathrm{c}$ is a plot of $\theta$ as a function of cut number corresponding to the time cuts of the EL onset distribution given in Figure 5.11b for $6 \mathrm{kV}$. Statistical errors are included but are not visible on the given scale. The two values at each cut represent the start and end points of the $\theta$ range. 

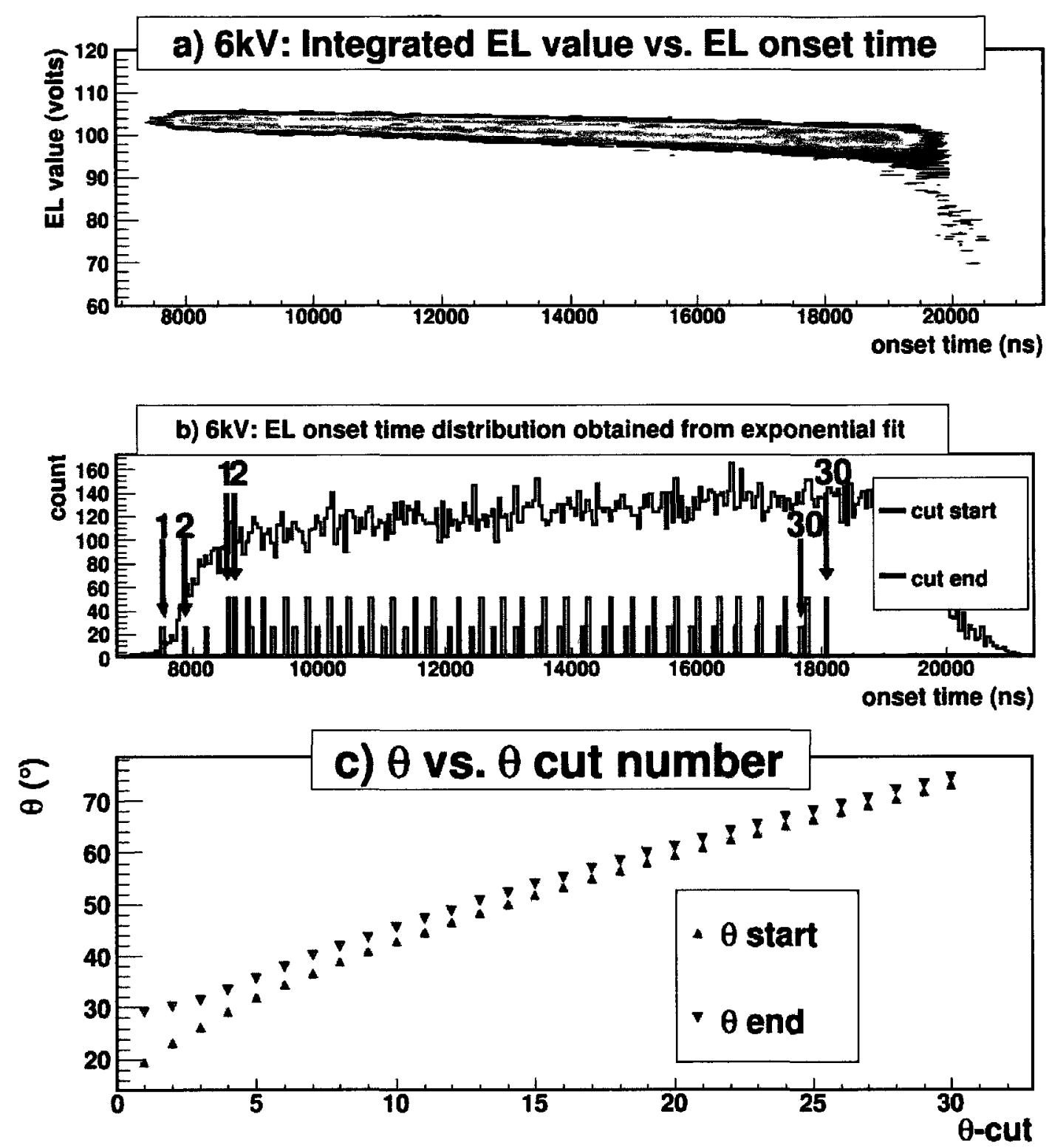

FIGURE 5.11: a) EL vs. EL onset time distribution. b) The corresponding onset time distribution showing the timing cuts ( 30 in total). Red signifies the cut start and blue the cut end. c) $\theta$ ranges corresponding to the timing cuts in b.

The scintillation and EL region average efficiencies corresponding to the obtained $\theta$ ranges for $6 \mathrm{kV}$ are presented in Figure 5.12. Figure 5.12 also gives the 
ratio of the scintillation to EL efficiencies used in Formula (3.9). The EL region efficiency plot exhibits a step like structure with eight semi-distinct regions. This is due to the segmentation of the efficiency map. The z-axis of the efficiency simulation was sectioned into $0.25 \mathrm{~cm}$ segments, resulting in 33 points ranging from $(-4.0 \rightarrow 4.0) \mathrm{cm}$. As $\theta$ increases incrementally, the $\alpha$ track's extent along the z-axis increases. However, the number of $\theta$ increments is larger than the number of efficiency divisions. Therefore a number of $\theta$-cuts correspond to the same EL efficiency region defined by the efficiency map, resulting in the same average efficiency for those cuts.

The EL efficiency as a function of voltage is given in Figures 5.13 for a selection of $\theta$ ranges. Average efficiencies are higher for small angle tracks. EL begins somewhere around $1.2 \mathrm{kV}$ and therefore the EL efficiency is essentially 0 below this value. EL efficiencies are given for voltages above $1.5 \mathrm{kV}$.

\subsection{Run-time Signal Stability}

The stability of the signals during data acquisition at a particular voltage was investigated and it was found that there was variation in the signals that correlated with temperature. The temperature was recorded at three positions in the lab, one at each PMT base and one at the crate containing the power supplies. Figure 

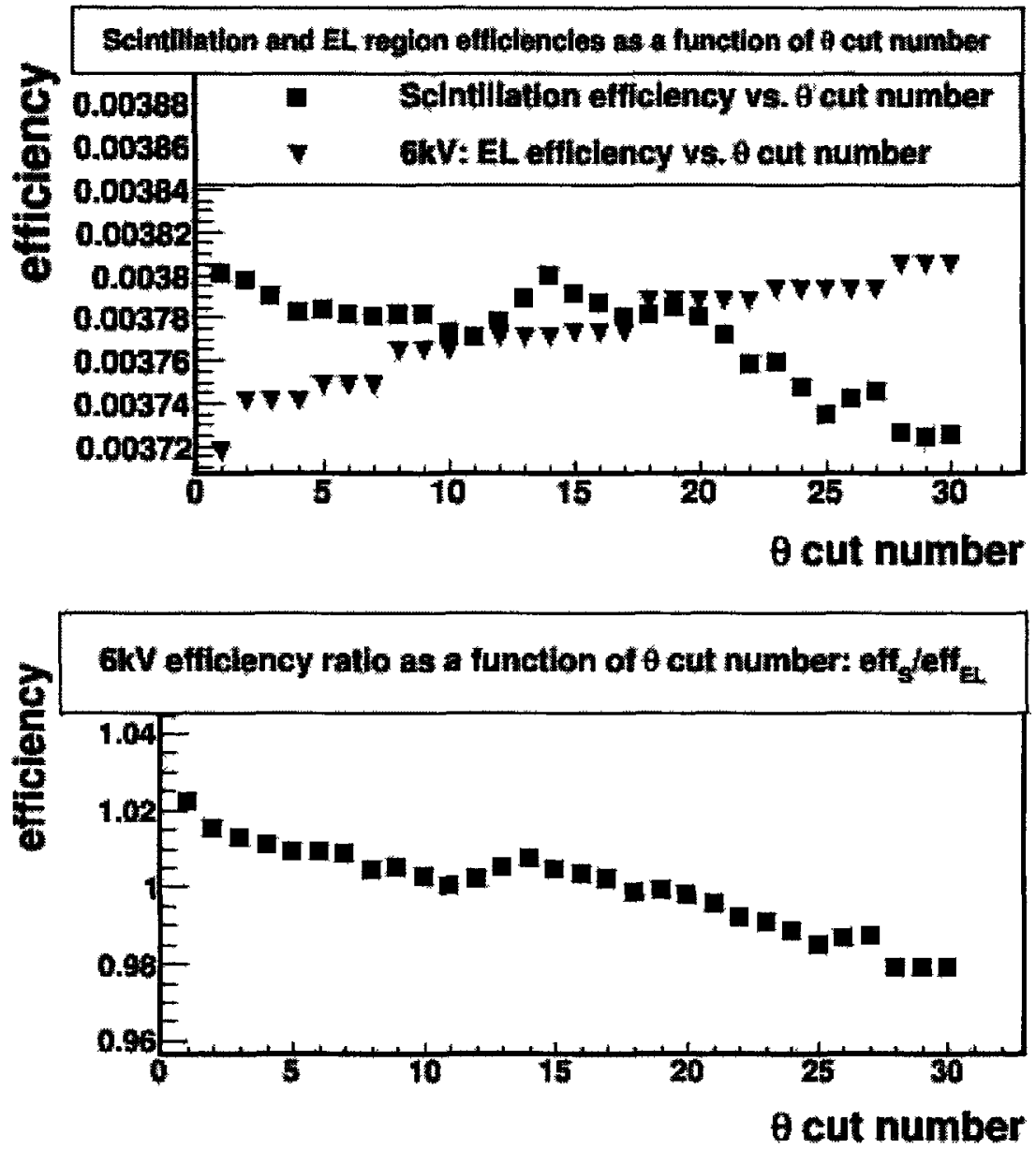

FIGURE 5.12: Efficiency of the scintillation and EL regions as a function of $\theta$-cut for $6 \mathrm{kV}$.

5.14 is a plot of the temperature as a function of time obtained during a $6 \mathrm{kV}$ run. The time axis represented by event number, where the event rate is $\approx 0.35$ Hz. The two readings taken at the PMT bases are essentially measurements of the room temperature, while temperature recorded at the crate is much higher due to heat given off by the power supplies. All three temperature readings follow the same dependence on event number, each showing a temperature change during run 


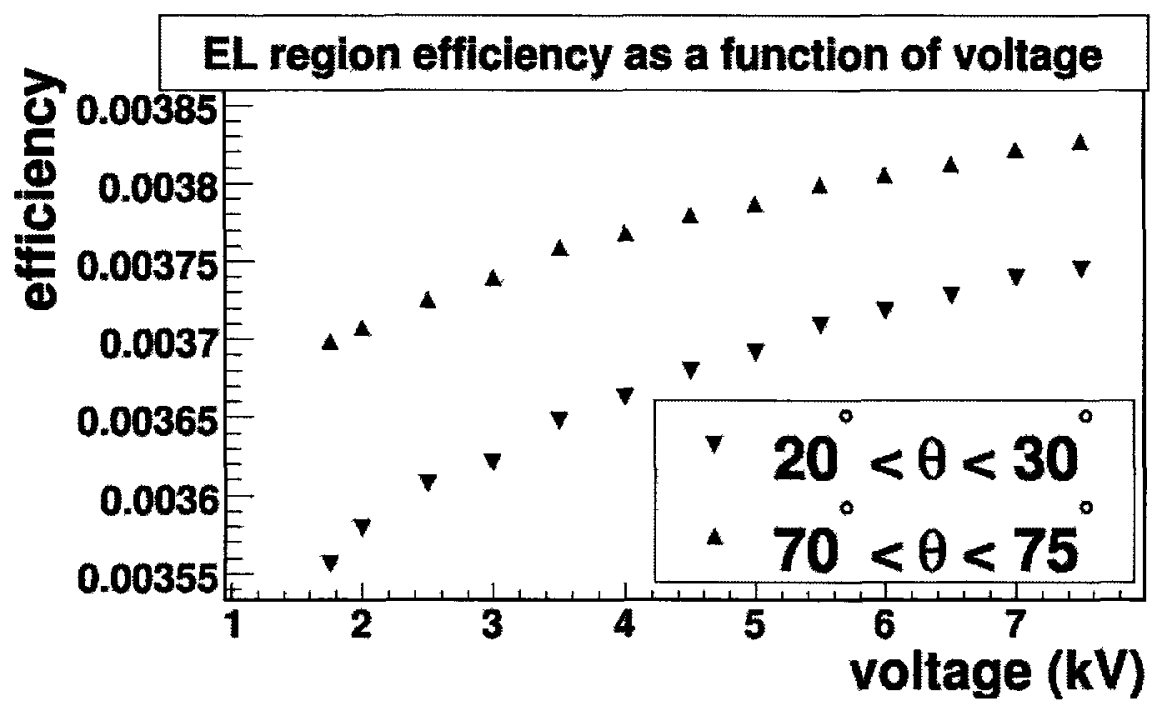

FIgURE 5.13: EL region efficiency vs. voltage: Angular dependence of EL efficiency for selected $\theta$-ranges.

acquisition of $\approx 4 \%$. The anode, scintillation, and EL signals versus event number obtained at a voltage of $6 \mathrm{kV}$ are given in Figure 5.15.

The variation of the mean of the EL signal with event number is apparent, with the signal roughly tracking the temperature changes but responding in the opposite sense. Anode and scintillation amplitudes are small, making temperature effects if present, harder to perceive in their respective distributions. To investigate the magnitude of the variation the data were divided into 500 event intervals and the mean of each determined. The mean, RMS and their ratio are plotted as a function event number in Figure 5.16. The same procedure was applied to the scintillation and anode signals. The maximum variations in the EL, scintillation, and anode signals are $\approx 2 \%, \approx 1.5 \%$, and $\approx 1 \%$ respectively. The variation in the 


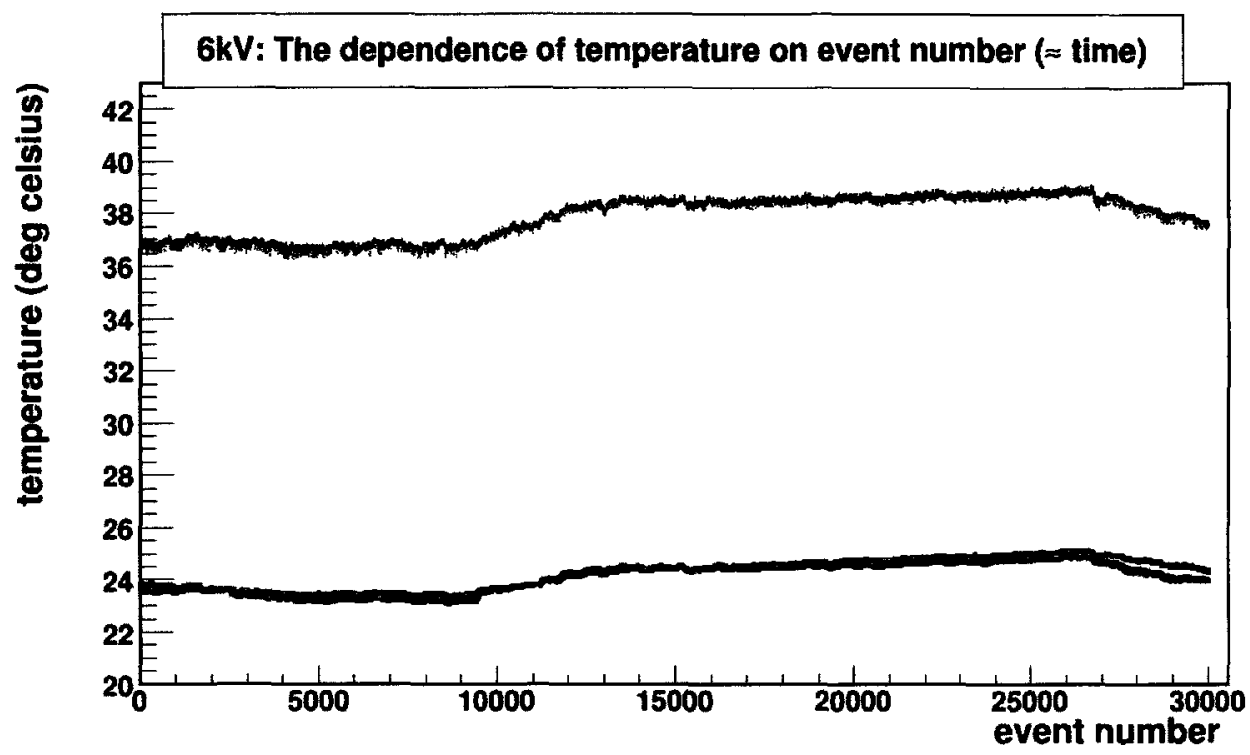

FiguRe 5.14: Temperatures measured at 3 representative positions in the lab. The time period is $\approx 16 \mathrm{~h}$ :

Bottom two curves PMTA \& PMTB, Top curve: Power supply rack
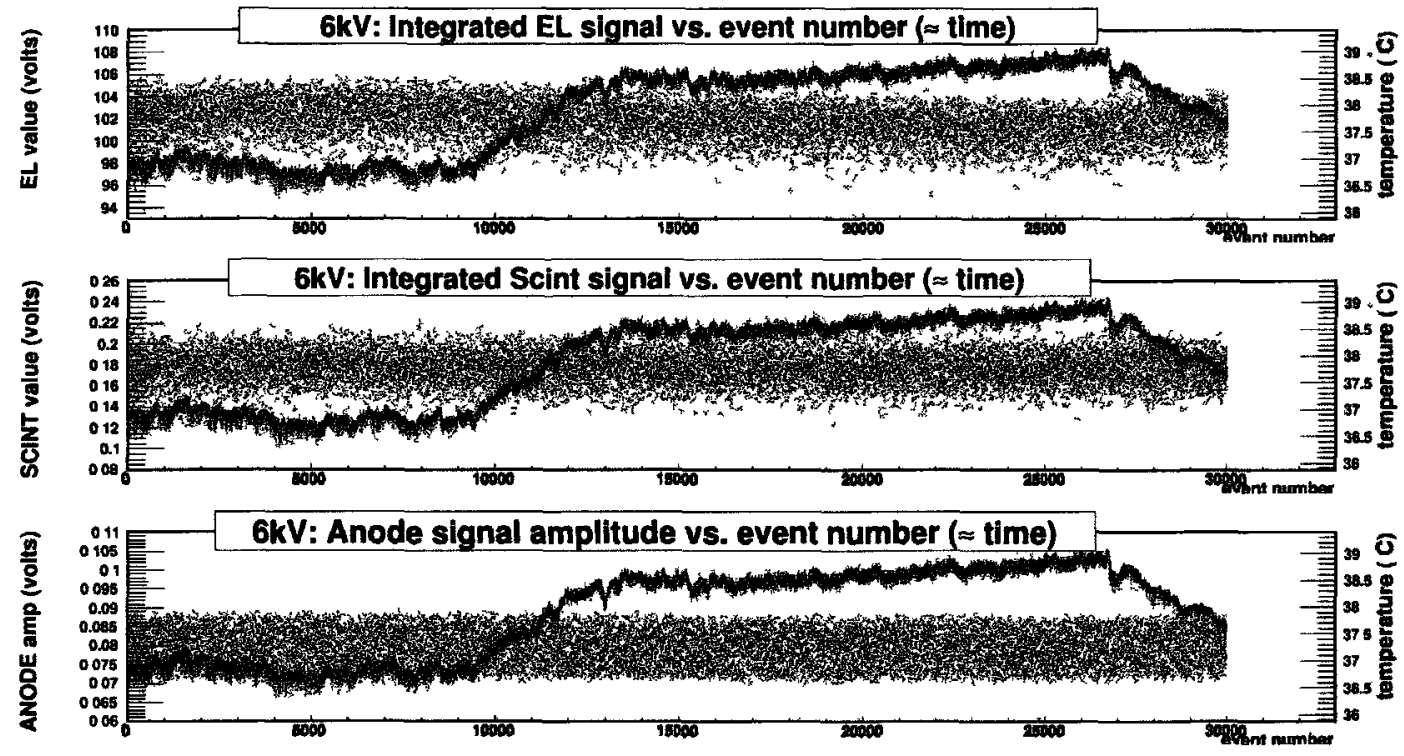

Figure 5.15: EL, scintillation, and anode signals vs. event number. Red: temperature, Black: Signal 
scintillation signal should not affect the measured resolution since it is expected to be above $(5-10) \%$. The $2 \%$ variation in the EL signal however will be a limiting factor in achieving a $1 \%$ resolution.

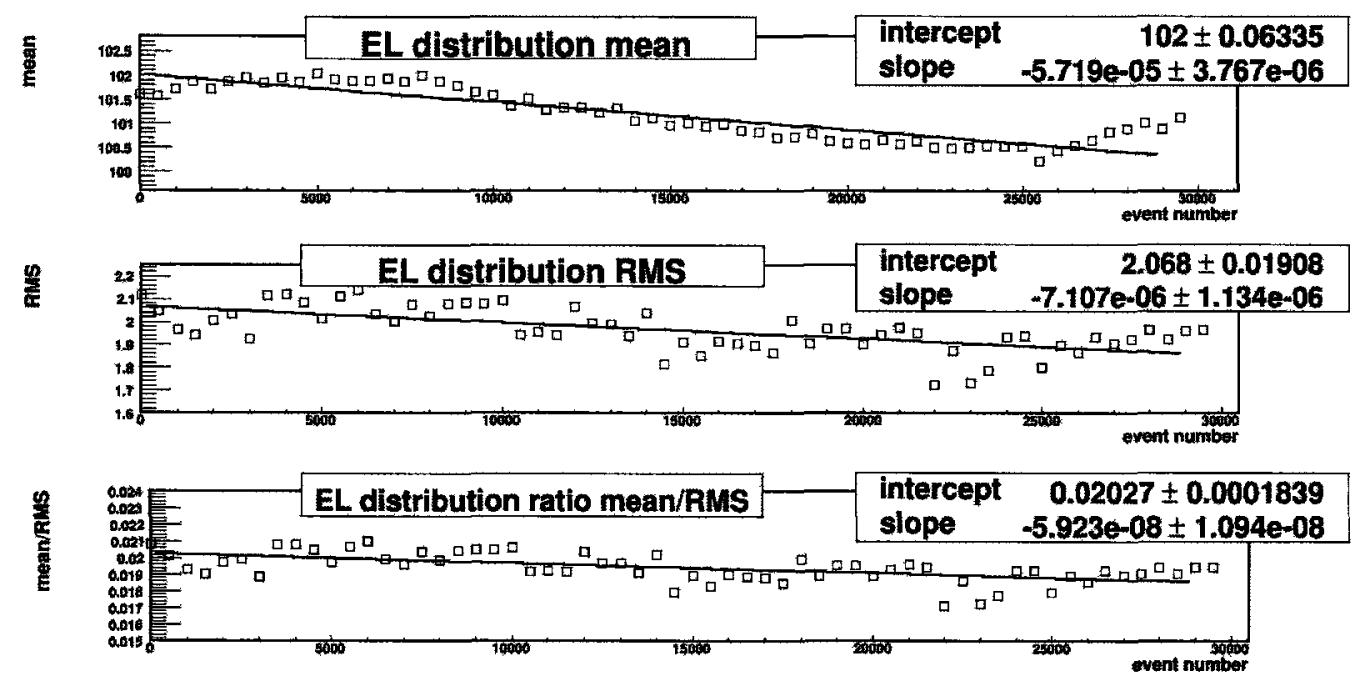

Figure 5.16: 1.) The mean of every 500 events for the EL signal 2.) The RMS of every 500 events for the EL signal 3.) RMS/mean ratio

A simple method was developed to correct the signals for temperature induced variations. The purpose of the correction is to examine the effects that the temperature induced signal variation have on the obtained energy resolutions. Oscillations in the signal are reduced by finding a scale factor for each event interval from the ratio of the mean value to the average of the entire data distribution. The data belonging to each event interval are then scaled and new distributions built. Figure 5.17 is the $6 \mathrm{kV}$ EL signal after the correction is applied. 
6kV: Corrected EL signal vs. event number ( $\approx$ time)

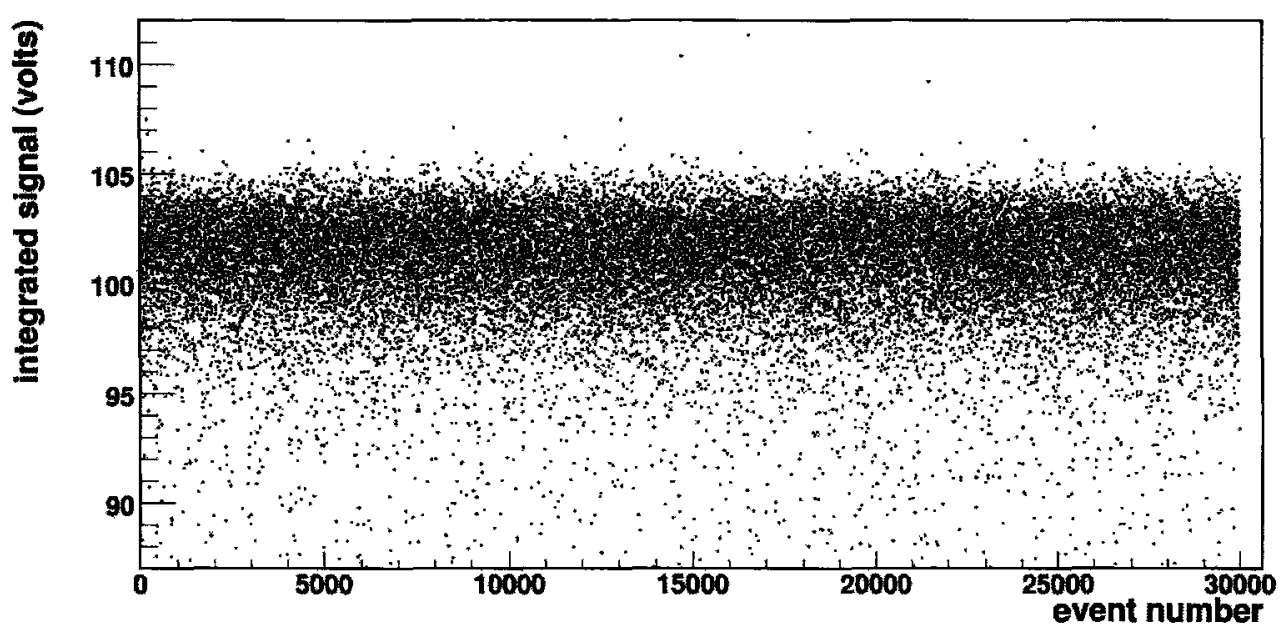

FIGURE 5.17: EL distribution corrected for temperature variations.

The temperature variations affect both the mean and rms of the EL signal (see Figure 5.18). The three plots in descending order were obtained for the event ranges, (0-6)k and (19-24)k, and (0-30)k respectively. All distributions are obtained for the same angular range $\left(\theta<45^{\circ}\right)$, each fit with a Gaussian excluding the low energy tail due to the large $\theta$-range. The angular range was chosen so the distributions obtained for the reduced event ranges contained at least 1400 events. There is a $1.4 \%$ shift in the mean and a $2.6 \%$ difference in the $\sigma$ 's for the uncorrected distributions obtained over the event ranges (0-6)k and (19-24)k, the resolution of the two distributions agree within $1.3 \%$. The resolution obtained for the entire event range is $\approx 16 \%$ larger than for the other two due to the signal variation with temperature. Figures 5.19 and 5.20 are the corresponding plots for the scintillation and anode signals. The scintillation signal does exhibit temperature 
induced variations but, due to the small amplitude, they are harder to perceive.

The resulting distributions show little difference in the Gaussian parameters. The anode amplitude has a much larger angular dependence than both the scintillation and EL values and the given $\theta$-range results in anode distributions which are less Gaussian as evidenced by the poor $\chi^{2} / n d f$ obtained from the fits. The key point for the anode signal is that the distribution parameters are in good agreement for all three event ranges indicating very little effect from signal oscillations.
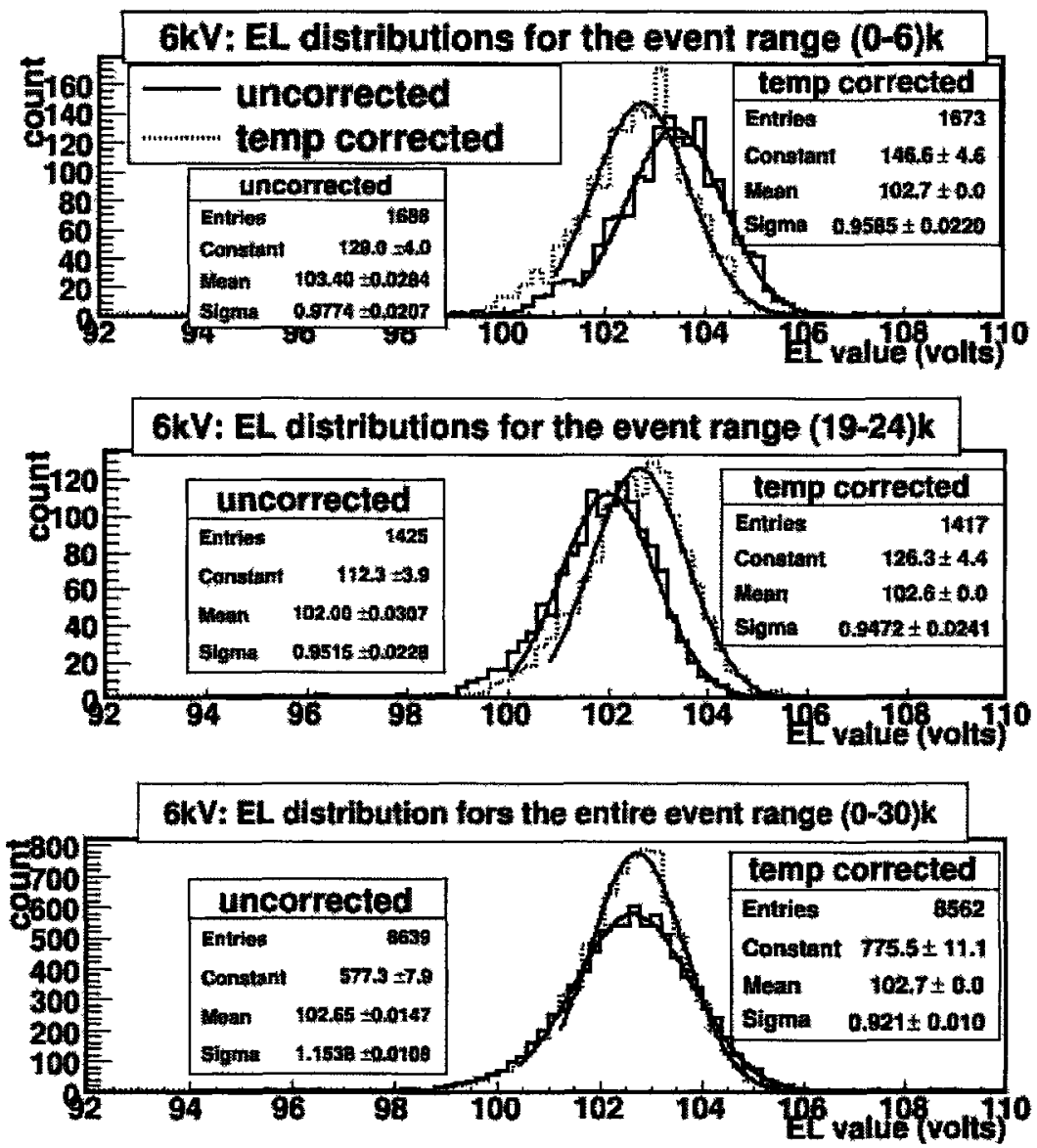

FIGURE 5.18: $6 \mathrm{kV}$ EL distributions obtained over various event ranges before and after temperature corrections. 

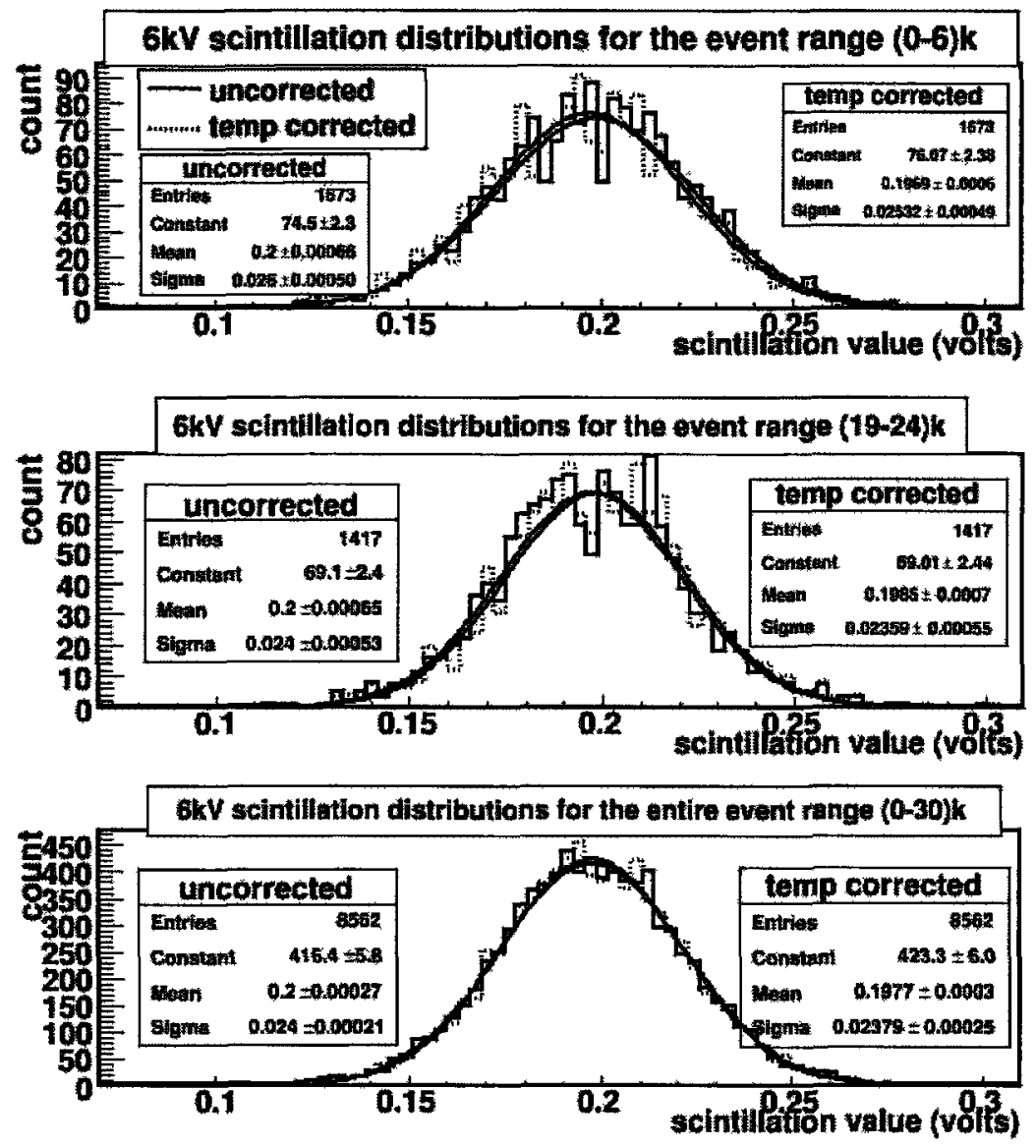

FIGURE 5.19: $6 \mathrm{kV}$ scintillation distributions obtained over various event ranges after temperature corrections.

The EL distributions before and after the correction is applied result in equivalent Gaussian means and $\sigma$ 's which agree within $4 \%$ for all three event ranges for the given angular range (see Figure 5.18). Therefore the use of the correction in making EL resolution measurements seems justified. While the scintillation signal also varies with temperature, the difference in the resulting distributions after temperature corrections is minimal. Since the scintillation resolutions are on the order of (5-10)\%, signal variations on the order of $2 \%$ should dramatically affect the 

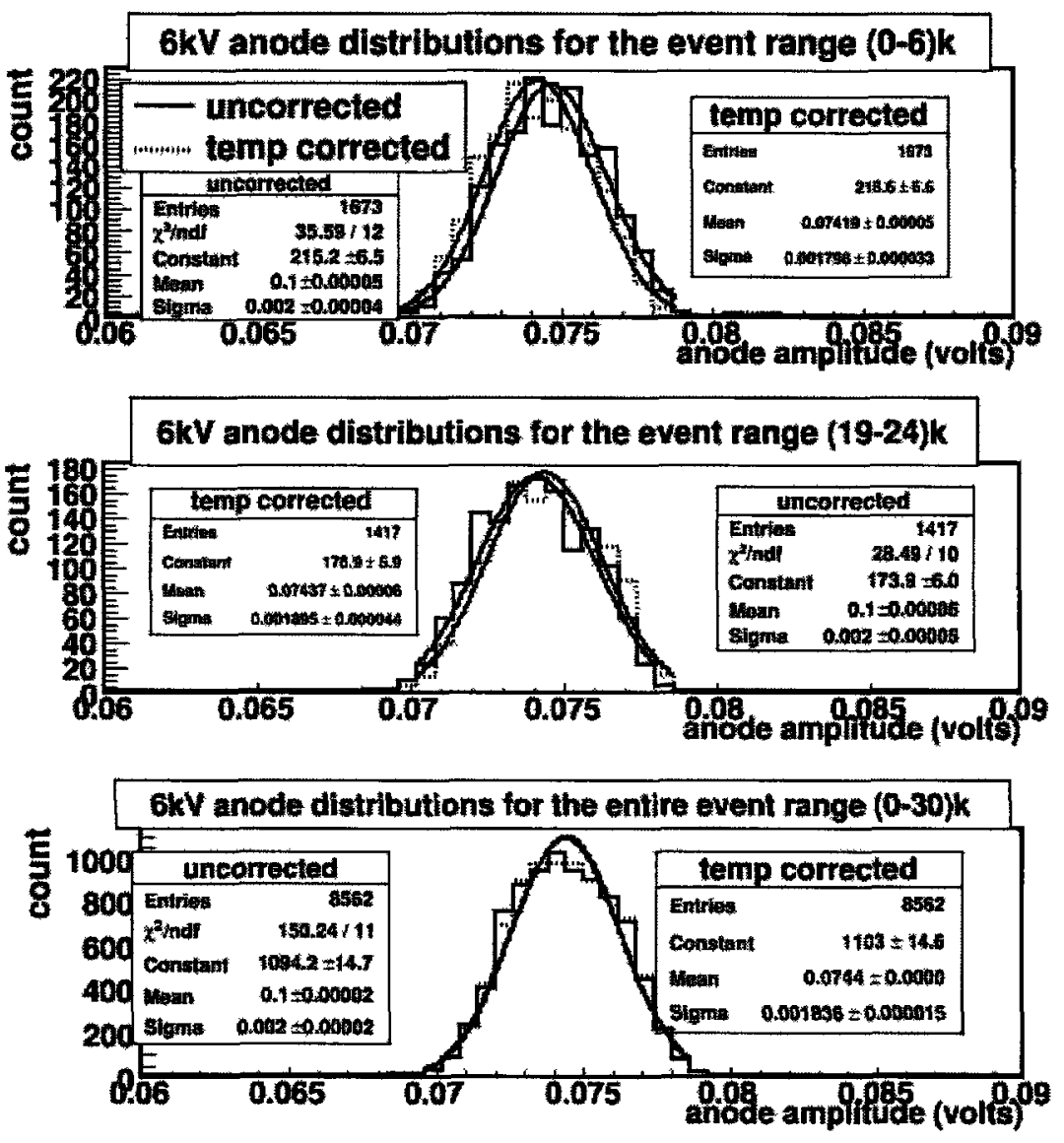

FIGURE 5.20: $6 \mathrm{kV}$ anode amplitude distributions obtained over various event ranges after temperature corrections.

resolution measurements. EL optical gain measurements rely on the ratio of the EL to scintillation signals and therefore the variation in the EL and scintillation signals should essentially cancel out resulting in a flat distribution. This will be addressed in the section on EL optical gain. Corrected distributions for the anode signal are essentially no different from the uncorrected ones (see Figure 5.20). Also there is little difference compared to the uncorrected resolutions, indicating that very little benefit will result by applying the correction to these quantities. 


\subsection{Response Stability During The Data Acqui- sition Period}

The last section addressed the stability of the signal during a single run, where it was found that the response was dependent on temperature. During the entire acquisition period the room temperature remained in the range $(22-26)^{\circ} \mathrm{C}$ and signal variations were on the order of $(1-3) \%$. These variations are small and independent of other factors which can affect the detector's response, such as, gas contamination due to a leak, virtual leak, out-gassing, or changes in the overall response of the PMTs. To monitor the stability of the detector over the data acquisition period. The ADMCA setup described in Section 3.1.2 was used to measure the average number of detected scintillation photons per ionization event at regular intervals.

The average number of scintillation photons produced is proportional to the energy deposited and so the $5.49 \mathrm{MeV}$ alphas produce, on average, the same initial number of photons. By measuring the average scintillation light yield daily the detector's response during the data acquisition period was monitored. Each day single photoelectron (SPE) and scintillation spectra were obtained. The SPE peaks were fit as described in Chapter 3 and Gaussian fits were applied to the scintillation peaks (see Figure 5.21). The yield was determined from the ratio of 
the obtained means. The values obtained from both PMT's were added together and plots of the resulting values versus day are given in Figures 5.22a,b, and c, for the scintillation and SPE means and the photon yield respectively. Both the gain and scintillation signals exhibit similar modulation which essentially cancels when taking their ratio. The fit to the sum number of photoelectrons results in a slope that is consistent with a straight line. indicating a stable detector response during the acquisition period. The average number of photons detected over the entire acquisition period is $434.2 \pm 6.4$. The given errors are systematic and were calculated assuming the PMT voltage error was taken to be half of the last digit taken from a multimeter used to monitor the PMT power supply, while the capacitance errors were taken as being $1 \%$ of the given value. The errors were then propagated through the calculations.

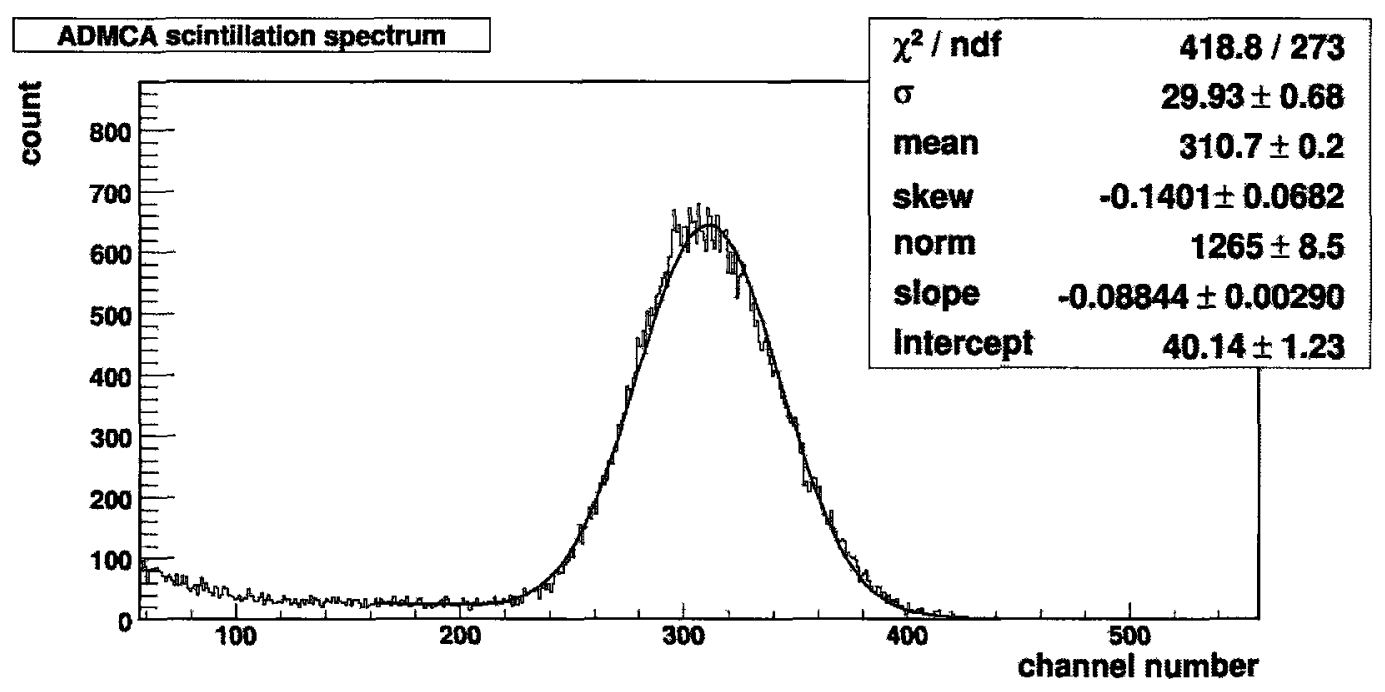

FIGURE 5.21: Scintillation spectrum obtained with ADMCA setup. Fit with a skewed Gaussian convoluted with a linear background function. 

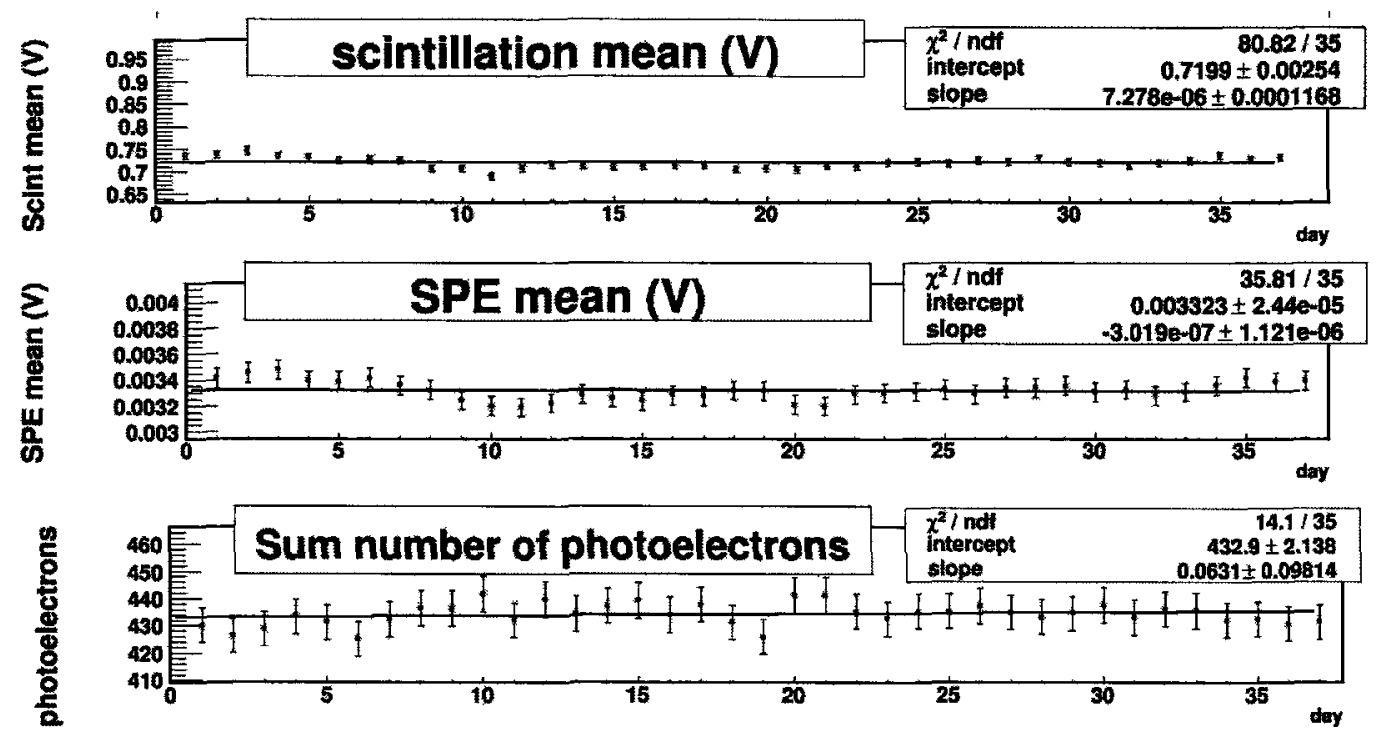

FIGURE 5.22: SPE and scintillation Gaussian means, and the total number of detected scintillation photons per event vs. day: Measurements obtained using the ADMCA setup. Systematic errors are presented.

\subsection{Anode signal analysis}

The analysis of the direct ionization signal collected by the anode provides a number of important pieces of information such as a measurement of the potential charge loss during transport and collection. The anode signal also provides an independent measurement of the source energy resolution and is utilized in making angular cuts for voltages below EL threshold.

Each alpha event ionizes the gas producing an amount of charge proportional to the deposited energy and the charge then drifts under the influence of an applied electric field until it is collected by the anode. The drifting charge induces currents in the anode, producing signal amplitudes that are proportional to the 
amount of charge and the distance travelled following Ramo's theorem [49]. The dependence of the anode amplitude on the $\alpha$ particle track angle arises from the different drift distance the electrons traverse as discussed. The track angle dependence due to the different drift distances is plainly apparent in examining a plot of anode amplitude as a function of EL onset time given in Figure 5.23 for $6 \mathrm{kV}$. The angular cuts of the previous section obtained from the onset time distribution were applied to the data and Gaussian fits performed on the resulting amplitude distributions.

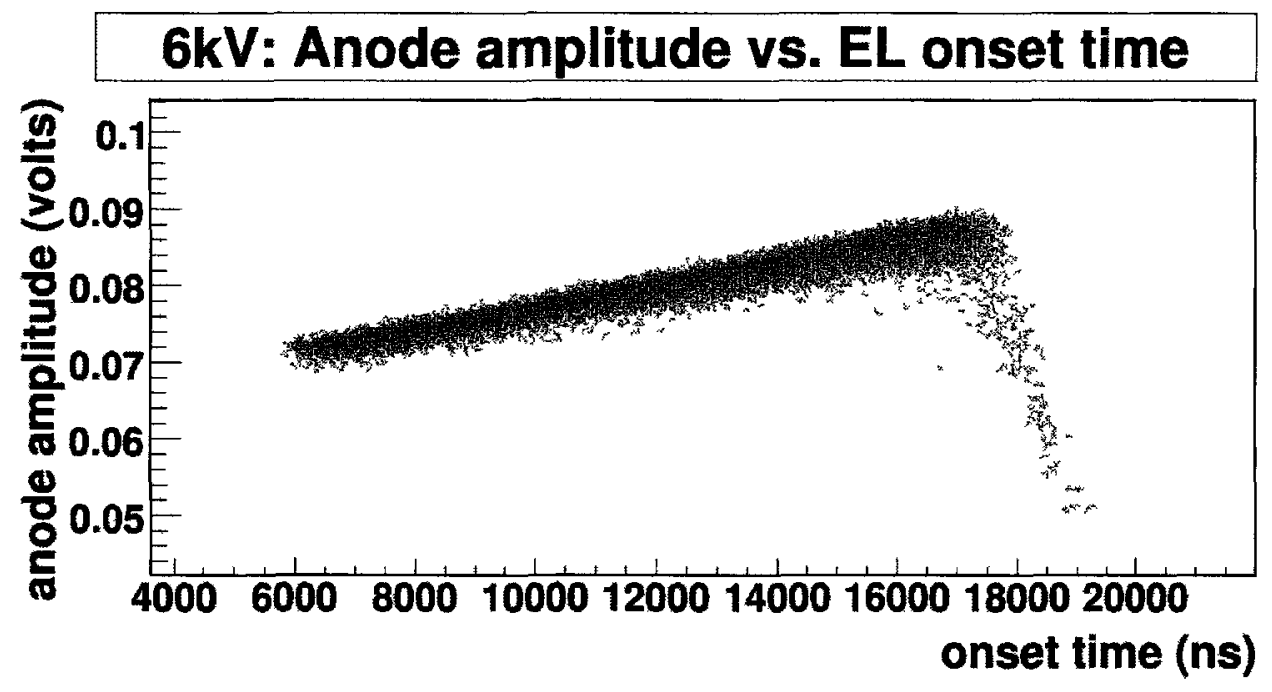

Figure 5.23: Anode amplitude vs. EL onset time.

The amplitude distribution obtained at $6 \mathrm{kV}$ for the angular range corresponding to the first $\theta$-cut is given in Figure 5.24. The amplitude mean versus operating voltage is given in Figure 5.25 for a selection of angular ranges. Errors 
are included in the plots but can not be seen on the given scale. The anode amplitude versus voltage exhibits a large angular dependence due to the different drift distances for parallel and perpendicular tracks (see Figure 5.25). The given plots have not been corrected for the decay time of the preamp. Corrections are applied which remove the angular dependence. A linear fit is applied to the amplitude versus onset time distributions and the slope is found as a function of voltage (see Figures 5.26). The fit is performed excluding the low energy tail due to range outs. Using the obtained fit parameters and the time corresponding to each angular region, a correction is applied for each $\theta$-cut. The resulting anode amplitudes agree over the entire angular range (see Figure 5.27). The slopes obtained below $2 \mathrm{kV}$ are less reliable and therefore the correction for these voltages is not perfect. This is because the EL pulses are not well defined at low voltages, resulting in a larger error in determining the correct onset time.

The anode amplitude is 0 at zero drift field and exhibits a very quick rise with voltage up to about $0.75 \mathrm{kV}$ at which point it approaches a limiting value. Past voltages of $\approx 0.75 \mathrm{kV}$ the rise of the amplitude is moderate with approximately a $14 \%$ increase over the range (see Figure 5.25). The increasing anode amplitude as a function of voltage over the range $\approx(0-0.75) \mathrm{kV}$ is due to the dependence of the recombination fraction on the electric field strength. At 0 field the probability for recombination is high but not $100 \%$, as some number of electrons will always escape recombination. As the field increases from 0 , the potential felt by an 


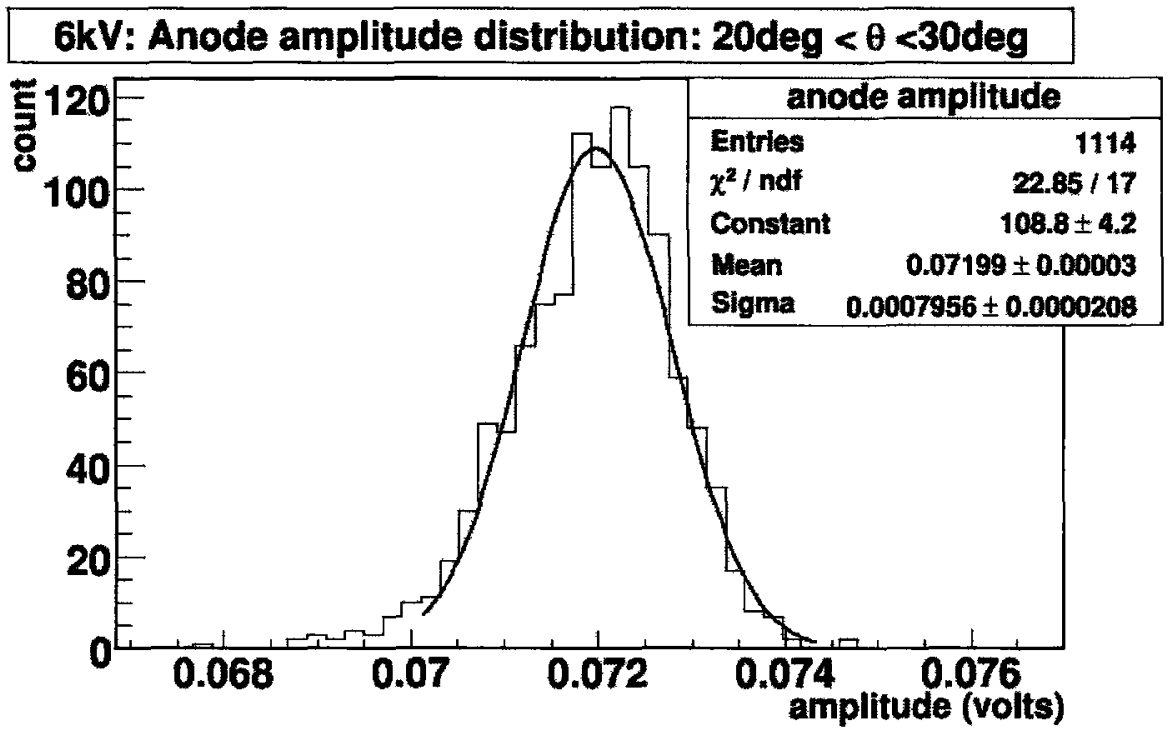

FIgURE 5.24: Gaussian fit to a $6 \mathrm{kV}$ anode amplitude distribution for the angular range: $20^{\circ}<\theta<30^{\circ}$.

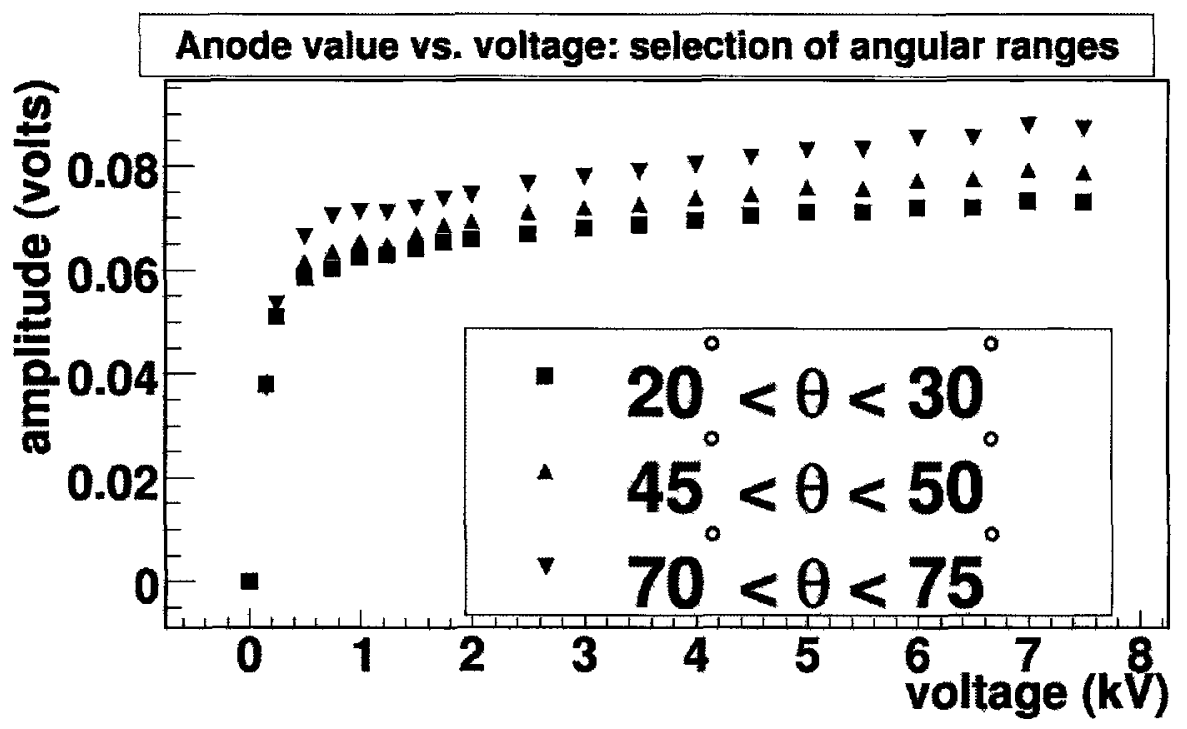

FIGURE 5.25: Anode amplitude vs. voltage for selected $\theta$-ranges showing angular dependence. Statistical errors are included but are not visible on the given scale. 


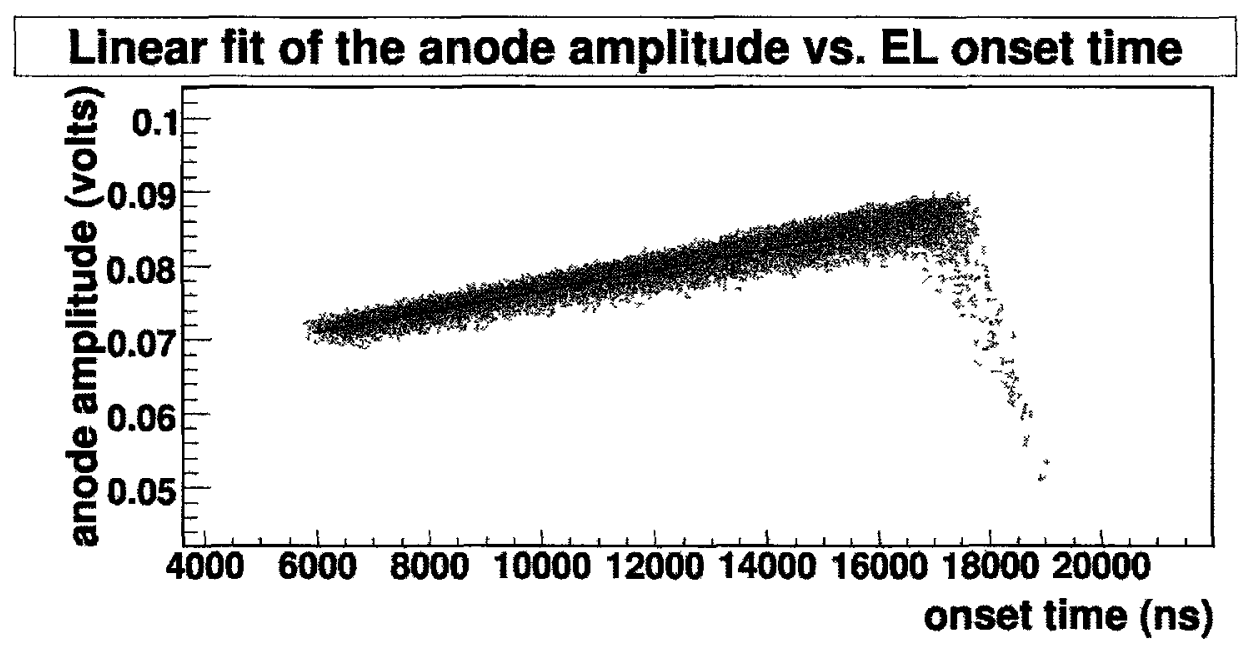

FIGURE 5.26: Linear fit applied to the anode amplitude vs. onset time.

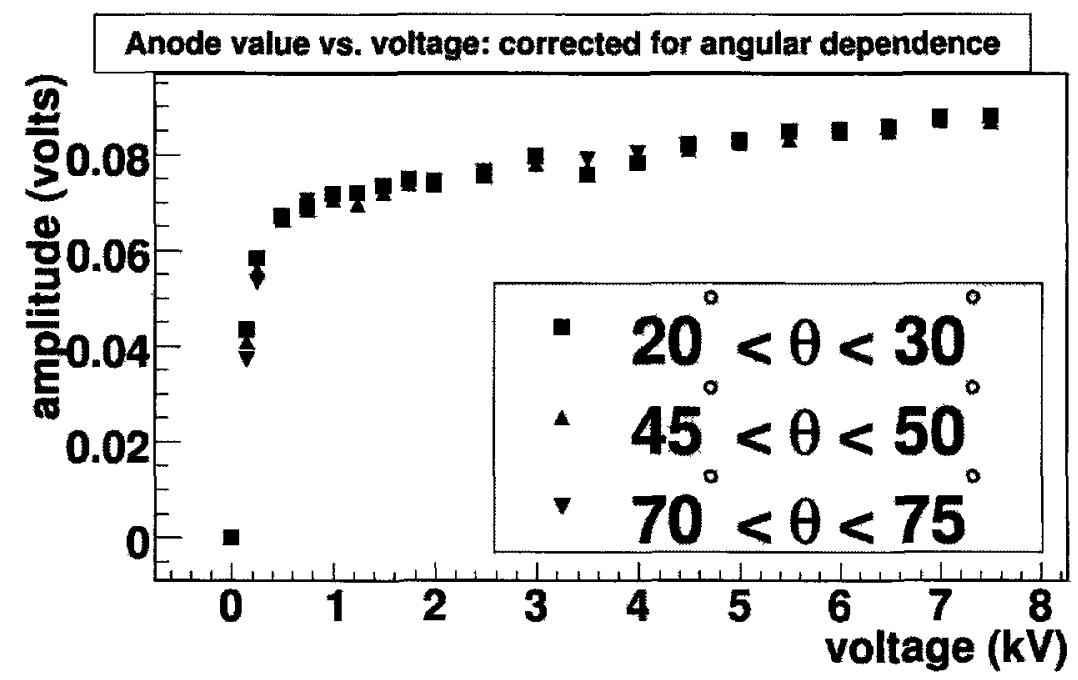

FIgURE 5.27: Anode amplitude corrected for angular dependence vs, voltage for selected $\theta$-ranges. The angular dependence is now removed.

electron/ion pair due to the field increases until it exceeds the coulomb potential preventing the majority of recombination. Above voltages of $1.75 \mathrm{kV}$ the potential is strong enough to allow for the collection of the majority of the produced charge. The increase in the anode amplitude after this point is due to the decay time of 
the preamp. The preamp time constant was measured to be $35 \mu s$ and was used to perform a amplitude correction for the exponential decay of the signal with time. The corrected amplitude as a function of voltage is given in Figure 5.28. After the correction there is an increase in amplitude and the distribution of amplitudes is essentially flat past $2 \mathrm{kV}$.

The preamp was calibrated by determining the response to a known input charge and determining a volt to electron conversion. By applying the conversion to the corrected anode values the number of ionization electrons as a function of applied voltage is found (see Figure 5.29). By fitting a constant to the voltage range (1.5-7.5) $\mathrm{kV}$ a value of $227900 \pm 1654$ collected ionization electrons is obtained. This value is $10 \%$ less then the expected number of electrons $\left(N_{\text {ion }}=E_{\alpha} / W_{\text {ion }}=5.49 \mathrm{MeV} / 21.9 \mathrm{eV}=250230 \pm 4488\right)$. This is a concern for making EL optical gain measurements. The introduction of the $\mathrm{W}$-value correction in the formula for EL gain (Equation (3.9)) assumes all of the charge produced drifts the entire distance through the EL region. If the discrepancy in the measured to expected number of electrons is attributed to charge loss due to capture by electronegative impurities, the amount of charge that drifts through the EL region is reduced, resulting in smaller EL amplitudes. The probability for a electron to be captured is proportional to the drift time and hence inversely to the drift velocity. Therefore the amount of charge lost is largest for low voltages. Assuming 
the level of impurities present does not affect the primary scintillation light production, the ratio of EL to scintillation values will be reduced, resulting in lower EL gain measurements. If the presence of impurities introduces added attenuation effects, scintillation and EL light will be affected equally.

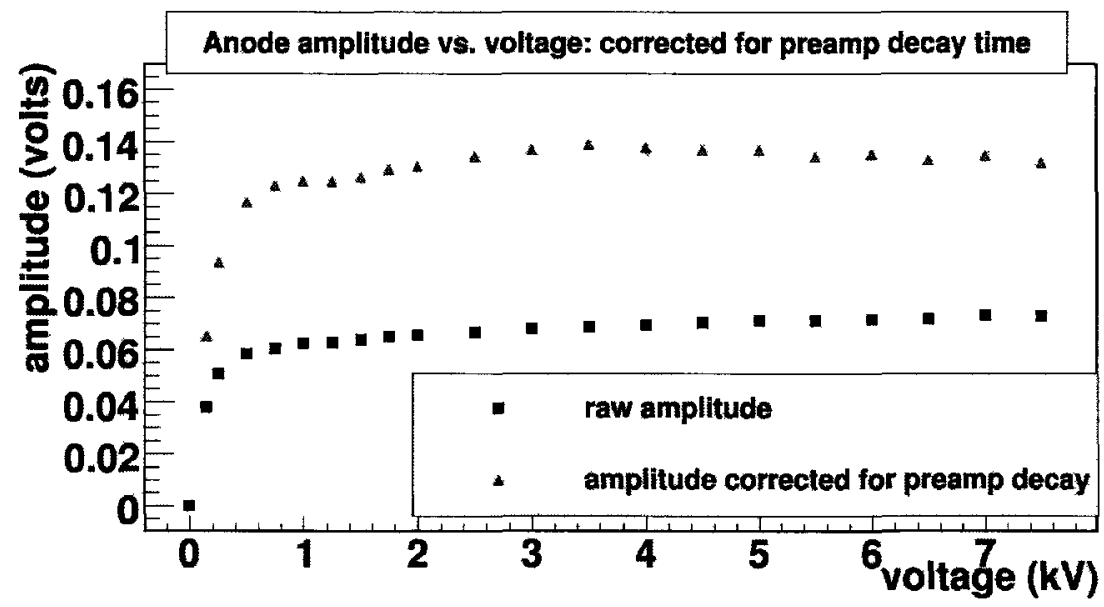

FIgURe 5.28: Anode amplitude vs. voltage: raw amplitude and decay time corrected amplitude. Statistical errors are included but are not visible on the given scale.

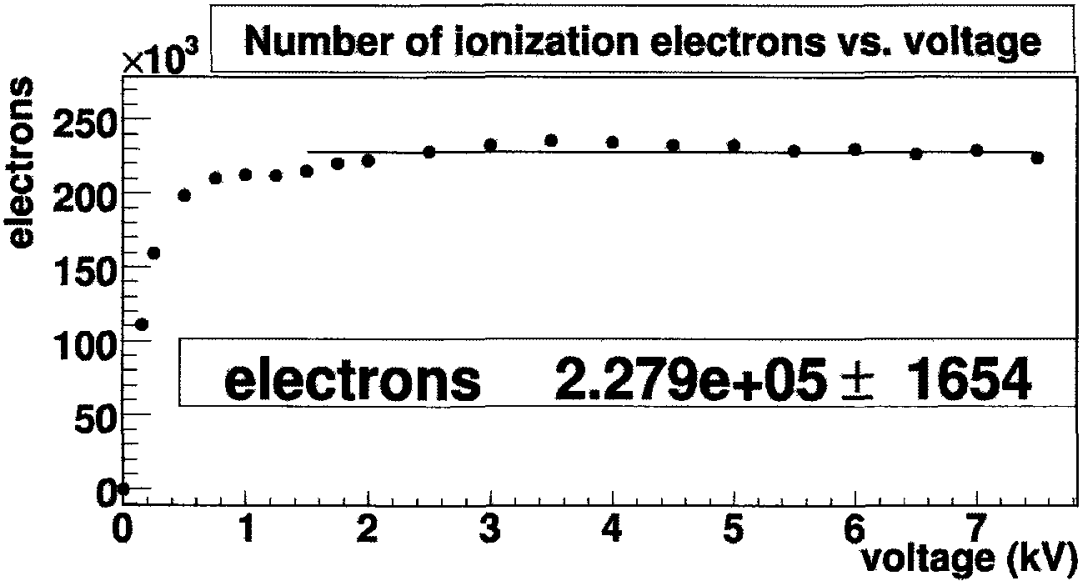

FIgURE 5.29: Number of ionization electrons collected. Constant fit applied above $1.5 \mathrm{kV}$ resulting in $\approx 228000$ electrons. 
The distribution of amplitudes is used in determining the energy resolution of the ionization signal, defined as $\delta E / E=\sigma_{\text {anode }} / M e a n_{\text {anode }}$. The Gaussian fit to the distribution of Figure 5.24 results in a resolution of $1.1 \pm 0.03 \%$. Figure 5.30 is a comparison of resolution curves corresponding to a selection of angular ranges. There is a clear angular dependence.

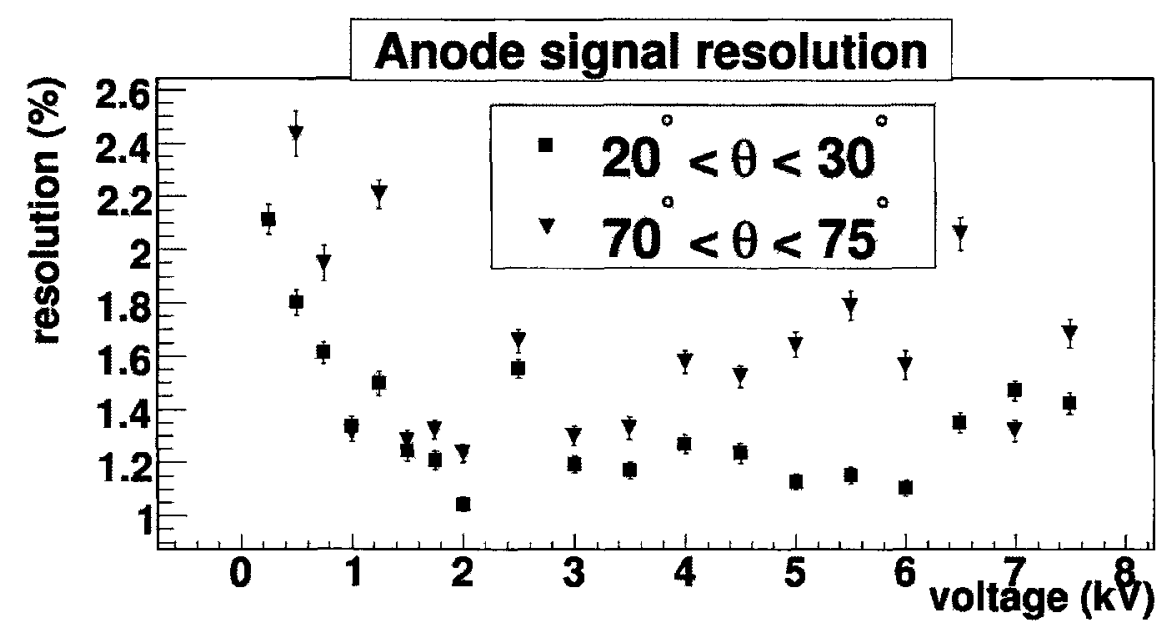

FIGURE 5.30: Resolution of the ionization signal for a selection of angular range.

The best ionization signal resolution achieved is $1.1 \pm 0.03 \%$, with values on the order of $1.3 \%$ over the entire angular range, for voltages above $3 \mathrm{kV}$. This level of energy resolution for the ionization signal is very good, especially considering the system was not optimized for direct charge measurements. The level of resolution achievable from the ionization signal is dependent on how accurately the amplitude of each anode signal is determined, and a noticeable feature of the anode signal is the periodic noise present throughout the trace at $\approx 4 \mu s$ time intervals. The 
detector was operated in the Carleton EXO lab where other experiments were operating and all electrical components were powered from the general Carleton electrical grid. While efforts were made to ensure the detector and all components were properly grounded, the exact source of the noise is not understood and all efforts to remove it were unsuccessful. The noise spikes introduce uncertainty in the position of the maximum and in the calculated average amplitude. This periodic noise is likely the dominant source of error, limiting the achievable resolution of the ionization signal. Temperature corrections were not applied to the anode signal based on the results of Section 5.2.

\subsection{Scintillation Signal}

The integrated scintillation amplitude was found by summing over the time region containing the pulse while subtracting the baseline bin by bin. The length of the signal integration window was defined by the expected time profile of the pulse, and a conservative window was chosen to ensure all the primary scintillation light was accounted for. The time interval for the window (T) was set as $T=\left(t_{0}-100 \rightarrow t_{0}+600\right) \mathrm{ns}$, with a second window set to $T=\left(t_{0}-100 \rightarrow t_{0}+300\right)$ ns. The implementation of a second integration window was based on preliminary results which indicated a dependence of scintillation amplitude on voltage. Two windows allow for a comparison of the resulting integrated values as a function of 
angle and voltage. Ionization track angle cuts were applied, and the integrated signal values were obtained from Gaussian fits to the resulting distribution. The distribution of integrated scintillation values obtained at $2 \mathrm{kV}$ corresponding to the two integration windows are given in Figure 5.31. The distributions were obtained for the angular range defined by the second $\theta$-cut $\left(\theta<30^{\circ}\right)$. The Gaussian parameters agree within $4 \%$ with smaller values corresponding to the $300 \mathrm{~ns}$ window. The resulting resolutions agree to better then $1 \%$. The $4 \%$ difference in the mean values is not unexpected. The expected time profile of the scintillation pulses obtained from the simulation indicates a minimum time window on the order (450-500) ns to allow for the full collection of the scintillation light, and that the choice of a 300 ns time window can result in a $\approx 3 \%$ loss in the number of collected scintillation photons.

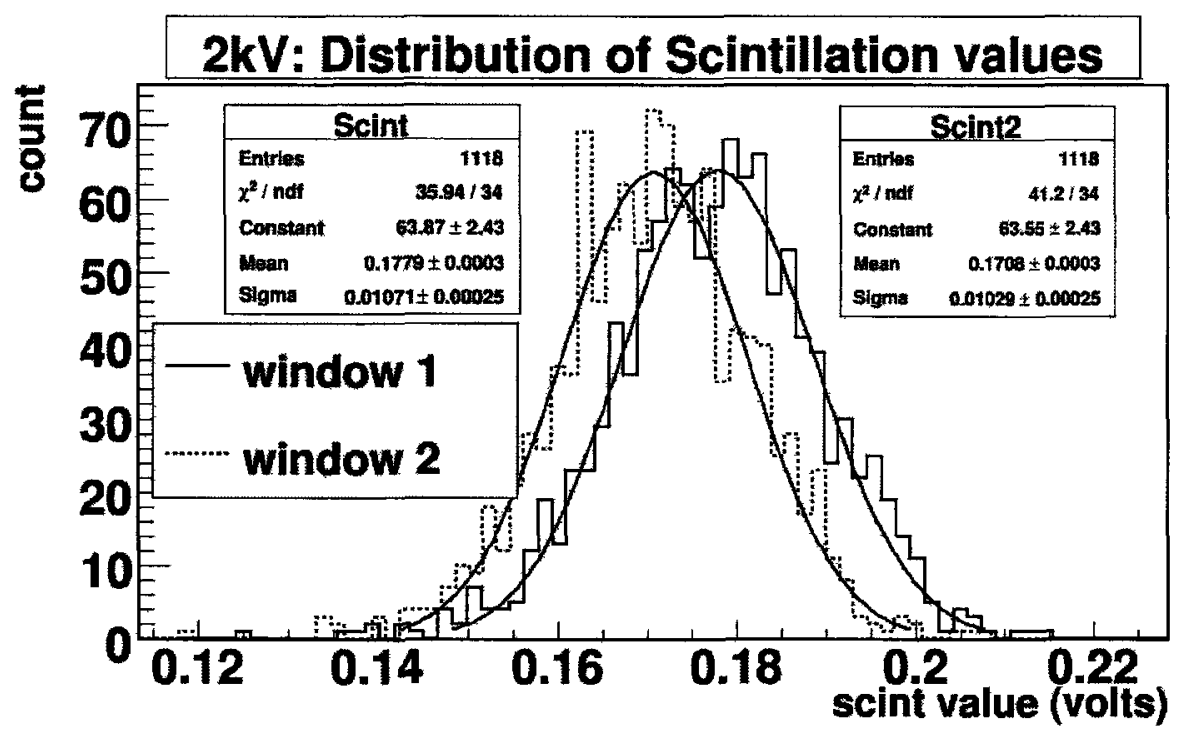

FIGURE 5.31: Gaussian fit to the scintillation distribution obtained at $2 \mathrm{kV}$. 
An anti-correlation exists between ionization and scintillation yields when recombination luminescence is present. The effect is well documented for LXe [50]. Recombination luminescence in xenon is highly dependent on the gas pressure, and previous experimental results reported that very few recombination photons contribute to scintillation in xenon at atmospheric pressure [31, 32]. Therefore, the scintillation yield is not expected to be largely dependent on the applied field, this is supported in the data where no correlation between the scintillation and ionization signals is observed. Since the average number of scintillation photons is directly proportional to the energy deposited in the gas, and not highly dependent on field at these pressures, the average scintillation value should be constant over the range of operating voltages, within the error introduced by the detection process. Fluctuations in the detector response include factors such as the during-run temperature induced signal variations, and the long term PMT gain response and stability. The error introduced by temperature induced signal variations is on the order of $2 \%$ over a single run, and the error associated with the PMT stability over the acquisition period is also on the order of $2 \%$. However, these two errors are likely correlated since changes in temperature affect the response the PMTs and the electronics. While a precise assessment of the expected error has not been performed, is not unreasonable to expect a variation on the order of $(2-5) \%$ in the mean scintillation values obtained from one run to the next.

The scintillation values obtained from the two time windows, corresponding 
to the angular range $\left(20^{\circ}<\theta<30^{\circ}\right)$, are plotted as a function of voltage in Figure 5.32. Above $5 \mathrm{kV}$ there is an obvious dependence of the scintillation value on voltage. Below $5 \mathrm{kV}$ the mean values agree within $6 \%$. This is a little larger then expected but not inconsistent with a flat distribution. The dependence on voltage is reduced for the $300 \mathrm{~ns}$ window. Since the drift time required for a perpendicular track to reach the EL region decreases with increasing voltage, the time between the scintillation pulse and the onset of EL also decreases. If the scintillation integration window is long enough and the drift time short enough, small amounts of EL light could be integrated with the scintillation light, artificially increasing the scintillation value and variance. Parallel tracks on the other hand have a long drift time compared to perpendicular tracks, and the time between the scintillation pulse and EL pulse is much longer then the scintillation time window. Therefore no EL light would be integrated with the scintillation light, distorting the mean and variance of the signal.

Plots of the scintillation values obtained from the two time windows versus voltage, corresponding to selected $\theta$-ranges, are given in Figure 5.33. Both time windows result in scintillation values which are dependent on voltage. The dependence is not nearly as pronounced for angular ranges with $\theta$ approaching $90^{\circ}$, and both distributions level off above voltages of $5 \mathrm{kV}$. Windowing and angular effects are considered to be the main contributing factor, but they do not fully explain the increase with voltage. 


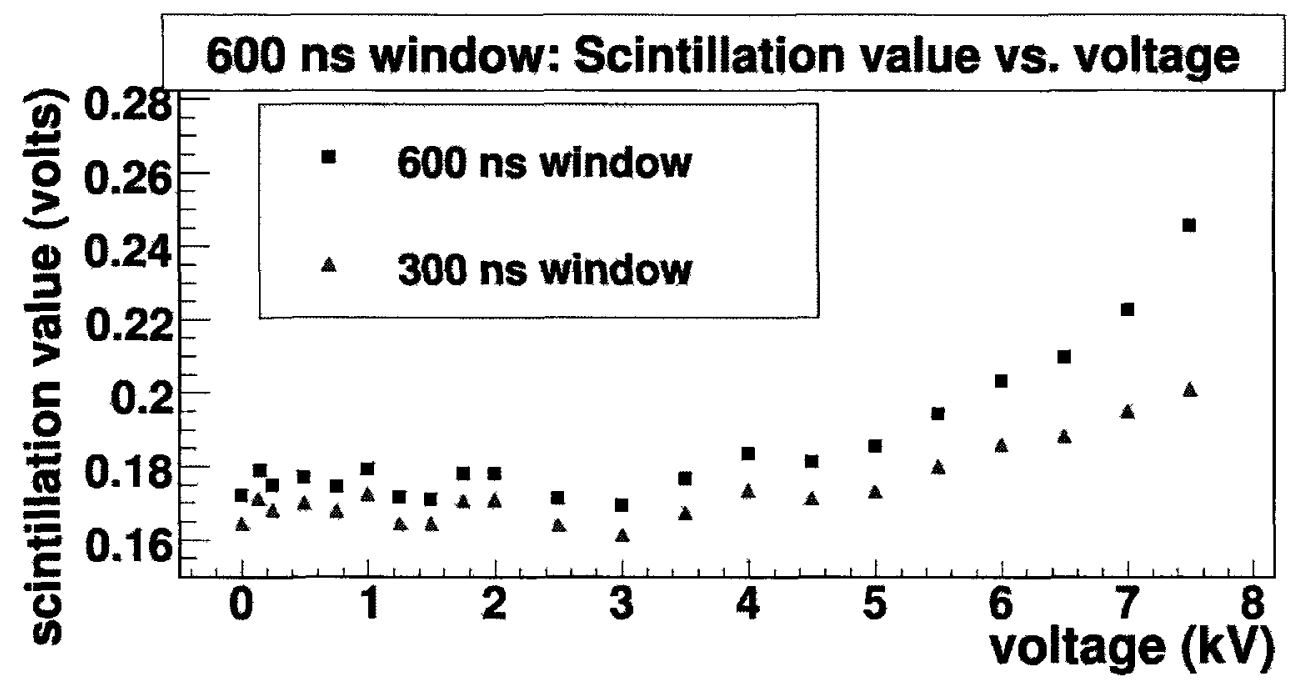

FIGURE 5.32: Scintillation mean vs. voltage for the angular range $\left(20^{\circ}<\right.$ thet $<_{30^{\circ}}$ ). Statistical errors are included but are not visible on the given scale.

The scintillation values obtained for the $600 \mathrm{~ns}$ and the $300 \mathrm{~ns}$ integration window are in $4 \%$ agreement with each other below $5 \mathrm{kV}$, with the $300 \mathrm{~ns}$ window exhibiting a smaller $\theta$ dependence. The $4 \%$ reduction of the scintillation value corresponding to the $300 \mathrm{~ns}$ time window is likely the result of a loss of collected scintillation light. This light loss will skew the EL gain measurements. Therefore the $600 \mathrm{~ns}$ window will be used. Also, since the increase in scintillation value above $5 \mathrm{kV}$ is believed to be artificial and not due to real light emitting processes, the average scintillation value taken over the voltage range $(0-5) \mathrm{kV}$ is adopted in making the EL gain measurements. Figure 5.34 gives the average scintillation value corresponding to the voltage range $(0-5) \mathrm{kV}$ as a function of angular range. There is a $\approx 3-4 \%$ difference in the values obtained for the two extreme $\theta$ ranges. 

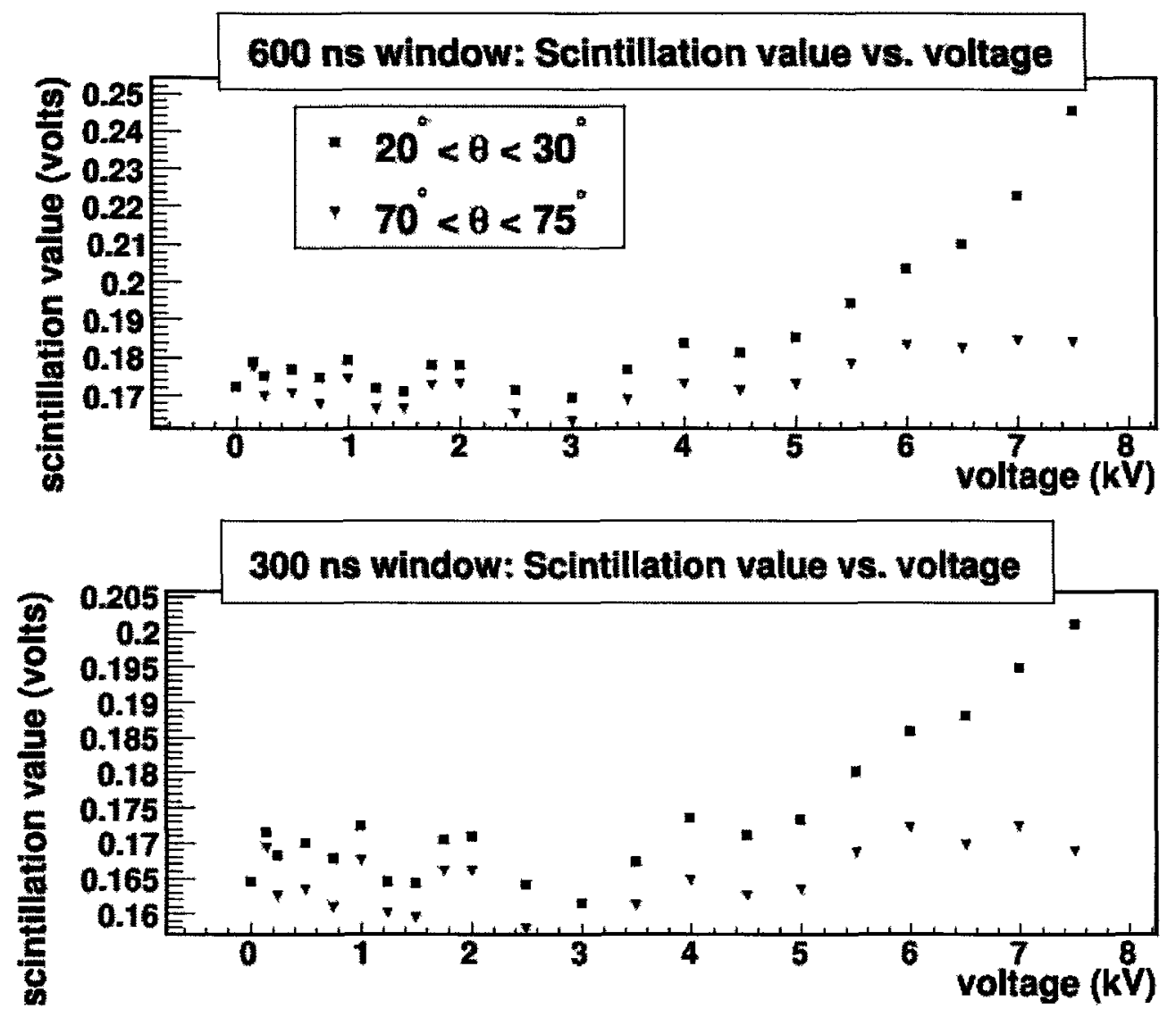

FiguRE 5.33: Scintillation values as a function of voltage corresponding to the two integration time windows for a selection of angular ranges. Statistical errors are included but are not visible on the given scale.

The decrease in scintillation value with increasing $\theta$, excluding range out events, is due to the contribution of two effects: energy loss due to the $\alpha$ traversing more source material, and the decrease in the efficiency for scintillation light collection with increasing $\theta$. The scintillation region efficiency corresponding to large $\theta$ ranges is on the order of $5 \%$ lower then for small angular ranges due to solid angle coverage (see Figures 5.11c and 5.12, indicating that efficiency effects are the dominant source of the decrease in scintillation value. 


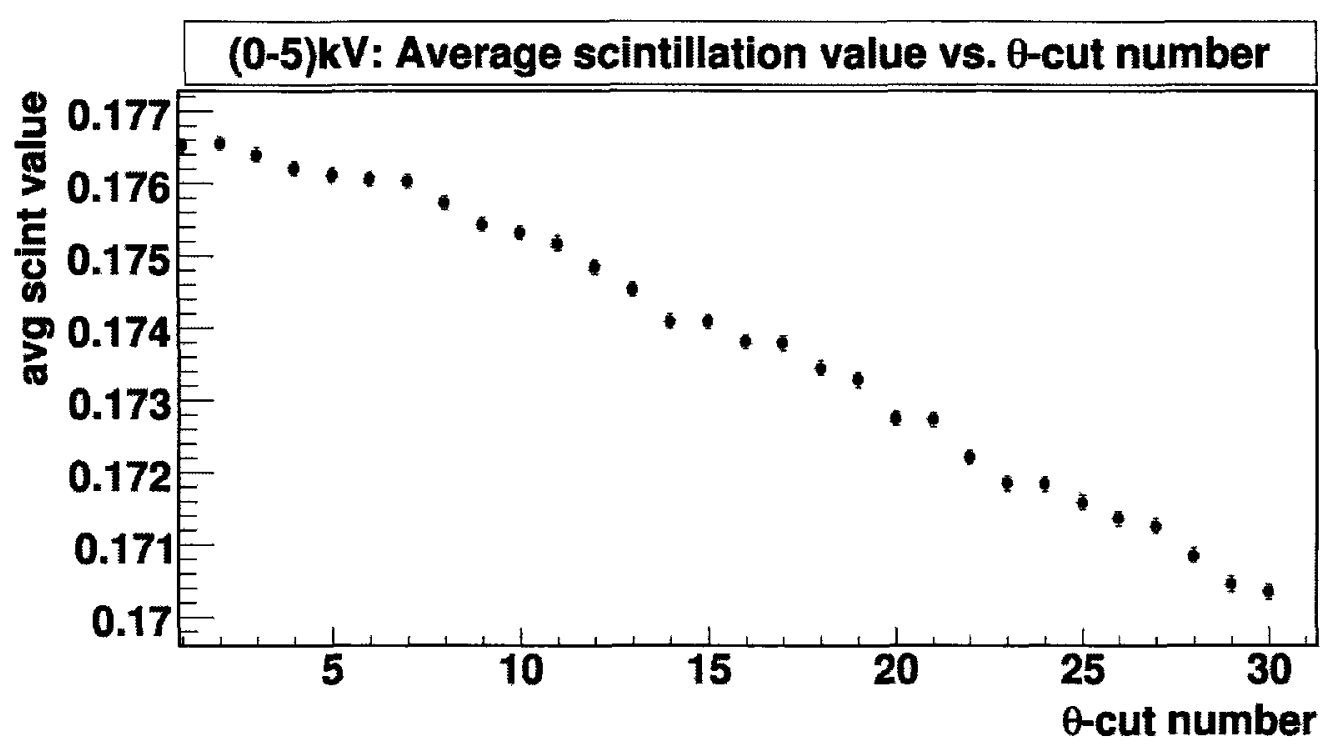

FIGURE 5.34: Average scintillation value taken over the voltage range (0-5) $\mathrm{kV}$ as a function of angular range ( $\theta$-cut).

The resolution of the scintillation signal is determined from the Gaussian parameters of the signal distribution. The resolution obtained from Figure 5.31 is $5.76 \pm 0.14 \%$. The resolution as a function of voltage corresponding to the 600 ns time window and the angular region $\left(\theta<30^{\circ}\right)$ is given in Figure 5.35. Below $5 \mathrm{kV}$ the resolution is fairly stable and on the order of (6-8)\%, at $6 \mathrm{kV}$ the resolution degrades suddenly and values obtained above $6 \mathrm{kV}$ are on the order of $(10-13) \%$. The mean and $\sigma$ of the scintillation distributions obtained at $6 \mathrm{kV}$ are $7 \%$ and $33 \%$ larger then those obtained at $5 \mathrm{kV}$ (see Figure 5.36), resulting in the large discrepancy in the measured resolution for two voltages. The change in the resolution above and below $5.5 \mathrm{kV}$ is correlated with the increase in the measured scintillation mean above $5 \mathrm{kV}$, lending support to the conclusion that 
non-scintillation light is being included in the integration window. The degradation in the resolution also seems to coincide with the increase in the RMS of the baseline distributions, indicating that fluctuations in the measured baseline value are introducing a corresponding variation in the measured scintillation signal.

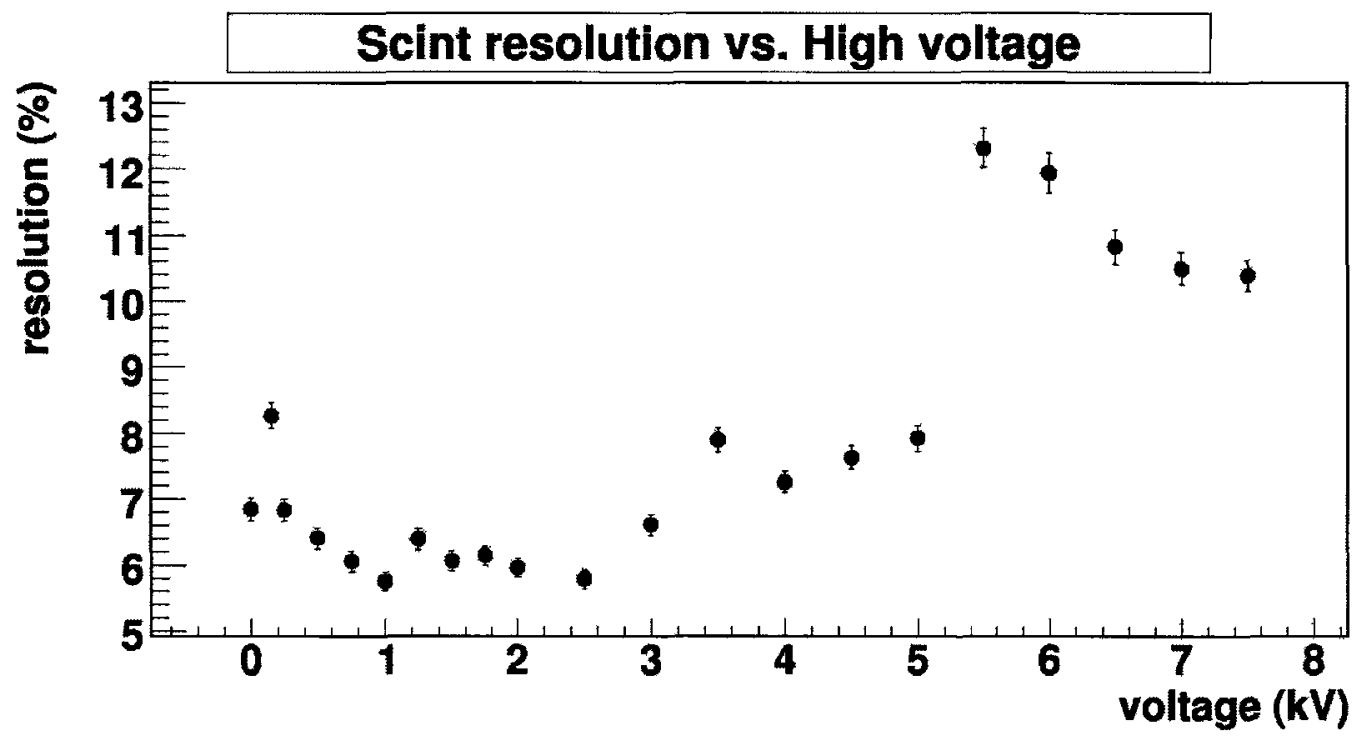

FIGURE 5.35: Scintillation signal resolution vs. voltage.

The resolution plotted as a function of angular range, for voltages of 2 , 4, and $6 \mathrm{kV}$ is given in Figure 5.37. The obtained resolutions are fairly consistent over the angular range, exhibiting a moderate dependence on $\theta$. The angular dependence is due to the fact that large $\theta$ tracks have lower efficiencies for light collection then do corresponding small $\theta$ tracks. The resolutions as a function of voltage corresponding to the two extreme angular ranges are given in Figure 5.38. The difference in the two resolutions at a particular voltage is on the order of $(5-7) \%$ over the entire range of voltages. The $5 \%$ difference in the efficiencies for 


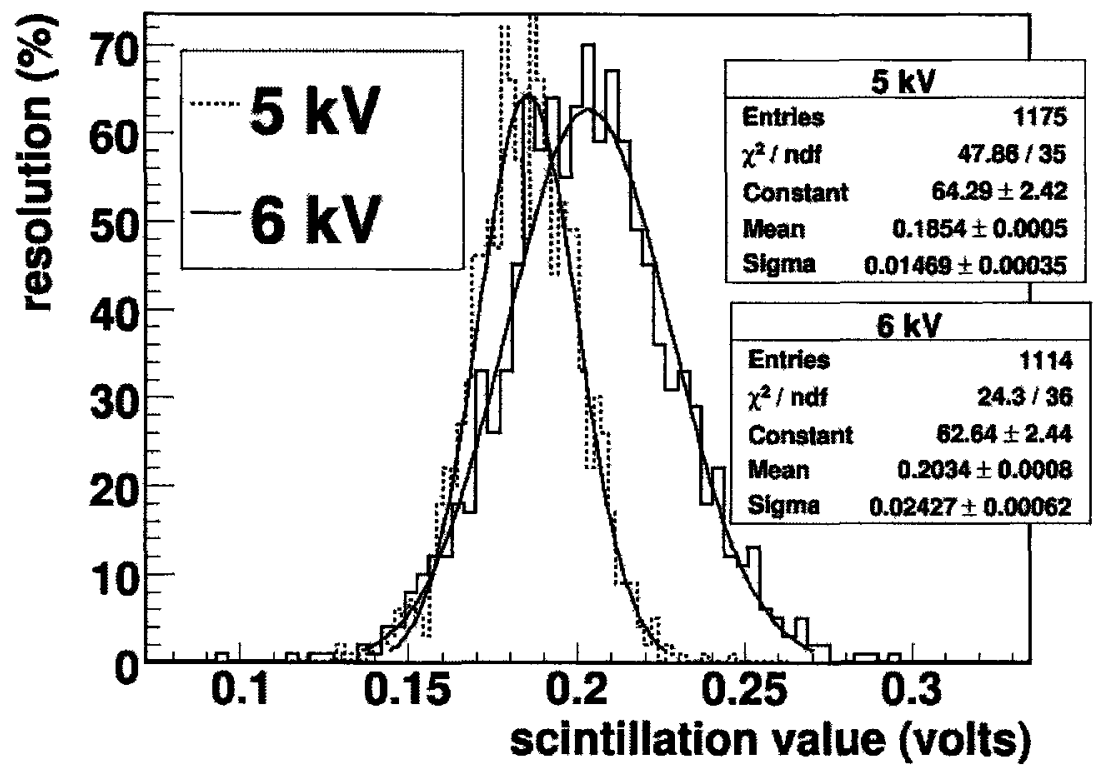

FIGURE 5.36: Scintillation distributions obtained at voltages of $5 \mathrm{kV}$ and $6 \mathrm{kV}$.

parallel and perpendicular tracks is likely the dominant cause for the different resolutions measured for the two angular ranges at a particular voltage. The results of Section 5.2 imply little improvement will result in resolutions from the application of temperature corrections. This is verified in comparing the resolutions as a function of voltage for corrected and uncorrected scintillation signals (see Figure 5.39).

Using the results from Section 5.4 for the number of detected photons per $\alpha\left(N_{p h}=434.2 \pm 6.4\right.$ photons $\left./ \alpha\right)$, the resolution expected from counting statistics 

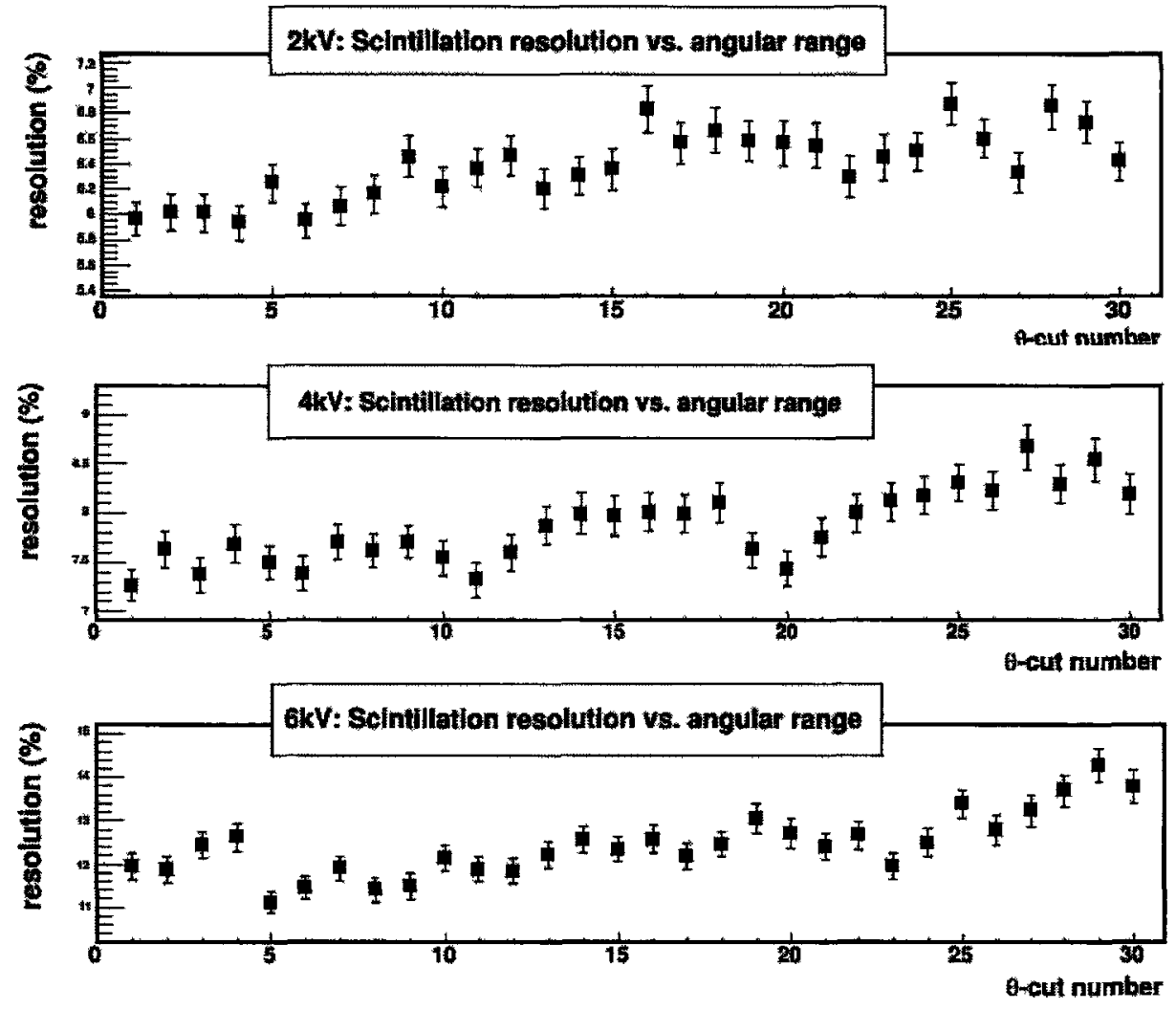

FiguRE 5.37: Plots of the scintillation resolution as a function of $\theta$-cut number for voltages of 2,4 , and $6 \mathrm{kV}$. The presented errors are statistical but there is correlation between neighbouring points due to overlap in the angular ranges.

is determined by,

$$
\text { resolution }(\%)=\frac{1}{\sqrt{N_{p h}}} \times 100 \%=4.799 \pm 0.007 \%
$$

The lowest scintillation resolution obtained is $5.76 \pm 0.14 \%$ at $1 \mathrm{kV}$, almost reaching the value predicted from counting statistics alone. The average resolution corresponding to the angular range $\left(\theta<30^{\circ}\right)$, taken over the voltage range $(0-5) \mathrm{kV}$, is $6.74 \pm 0.16 \%$. There are a number of factors which can degrade the scintillation 


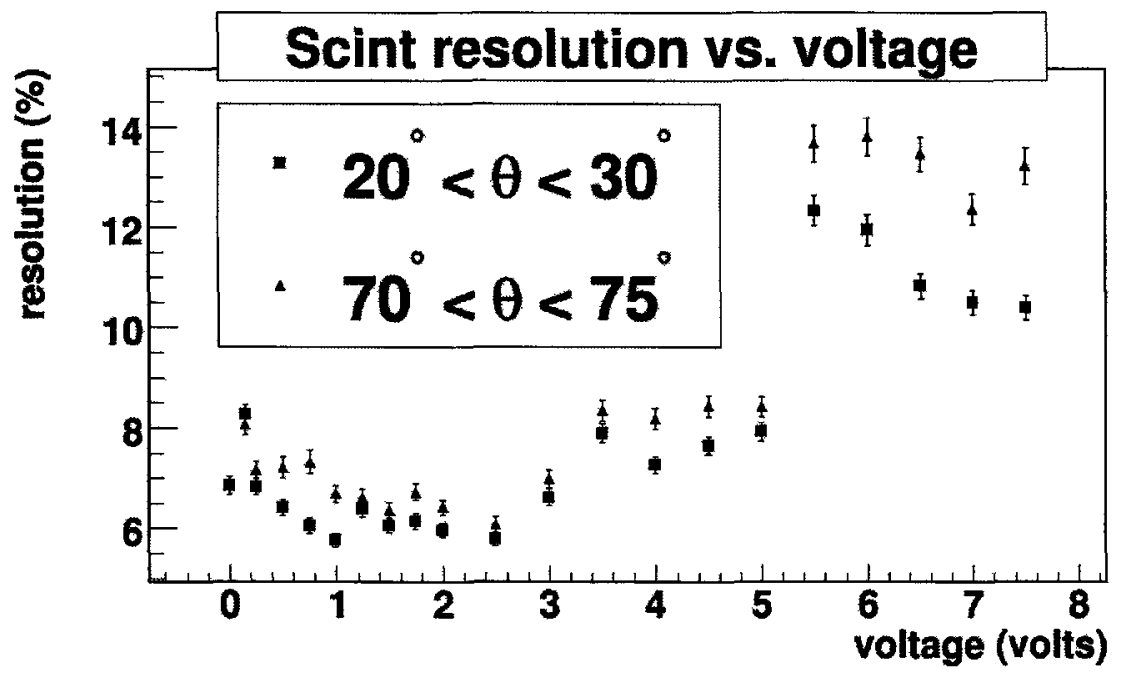

FIGURE 5.38: Scintillation resolution vs voltage corresponding to the two given $\theta$-ranges.

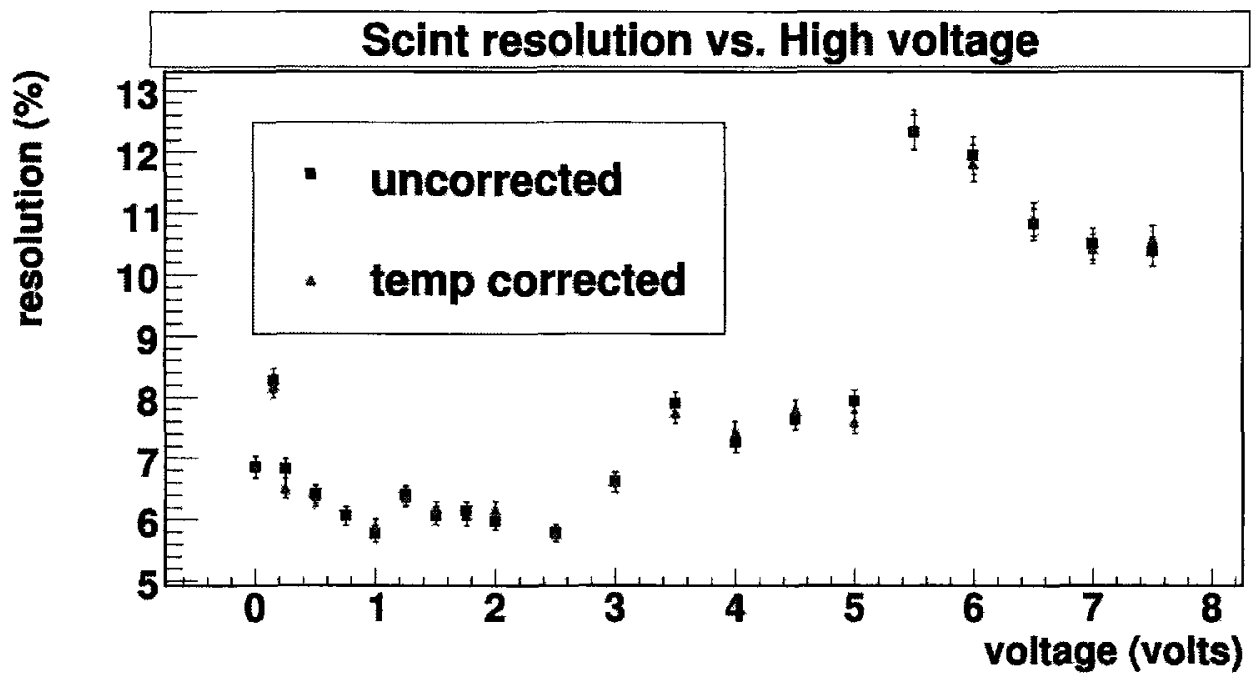

FIGURE 5.39: Comparison of temperature corrected and uncorrected scintillation resolutions.

resolution, such as intrinsic fluctuations in the scintillation process represented by a Fano like factor, response fluctuations of the PMTs, and fluctuations in the conversion process of the WLS. These are the likely cause of the discrepancies in 
the measures and expected values.

Using the average efficiency for the scintillation region obtained from the simulation $\left(S C I N T_{\text {eff }}=0.00373 \pm 1.39 \times 10^{-5}\right)$ the total number of scintillation photons per $\alpha$ can be estimated as,

$$
N_{s}=\frac{N_{p h}}{S C I N T_{\text {eff }}}=116400 \pm 1770 \text { scintillation photons }
$$

From the obtained number of scintillation photons per $\alpha$ an estimate of $W_{s}$ can be obtained from Equation (2.4). The resulting $W$-value is $W_{s}=47.2 \pm 0.7 \mathrm{eV}$. The obtained value is not at all consistent with the value of M. Mimura et al. $\left(W_{s}=34.6 \pm 1.6 \mathrm{eV}\right)[27]$, but is in agreement with the value of M. Miyajima et al. within two standard deviations $\left(W_{s}=49.6 \pm 1.1 \mathrm{eV}\right)$ [32]. The method used for obtaining $W_{s}$ is not precise. The result however, does indicate that the use of the larger of the two $W_{s}$ values in the EL gain correction is appropriate for the analysis. The use of $W_{s}=49.6$ is also supported by the results of the scintillation simulation, where by assuming $W_{s}=49.6 \mathrm{eV}$, the distribution of the expected number of scintillation photons detected resulted in a mean of $417.4 \pm 0.5$ with a standard eviation of $21.43 \pm 0.36$. There is a $4 \%$ discrepancy in the number of detected photons obtained from the ADMCA calibration and the simulation $\left(W_{s}=49.6 \mathrm{eV}\right)$, with the two values agreeing within 3 standard deviations of the 
error on the ADMCA measurements.

\subsection{EL Signal and Optical Gain}

The integrated EL signal is obtained by summing over the EL pulse bins while subtracting the calculated baseline bin by bin. A conservative integration window was set using the expected trace and EL pulse timings obtained from the simulation. The following results were obtained using this window. An assortment of other integration windows were investigated, allowing for the comparison of final results obtained from the different integration windowing's. This gives a rough estimate of the error introduced by the choice of integration window. Angular event cuts were applied, and the Gaussian parameters determined (see Figure 5.40).

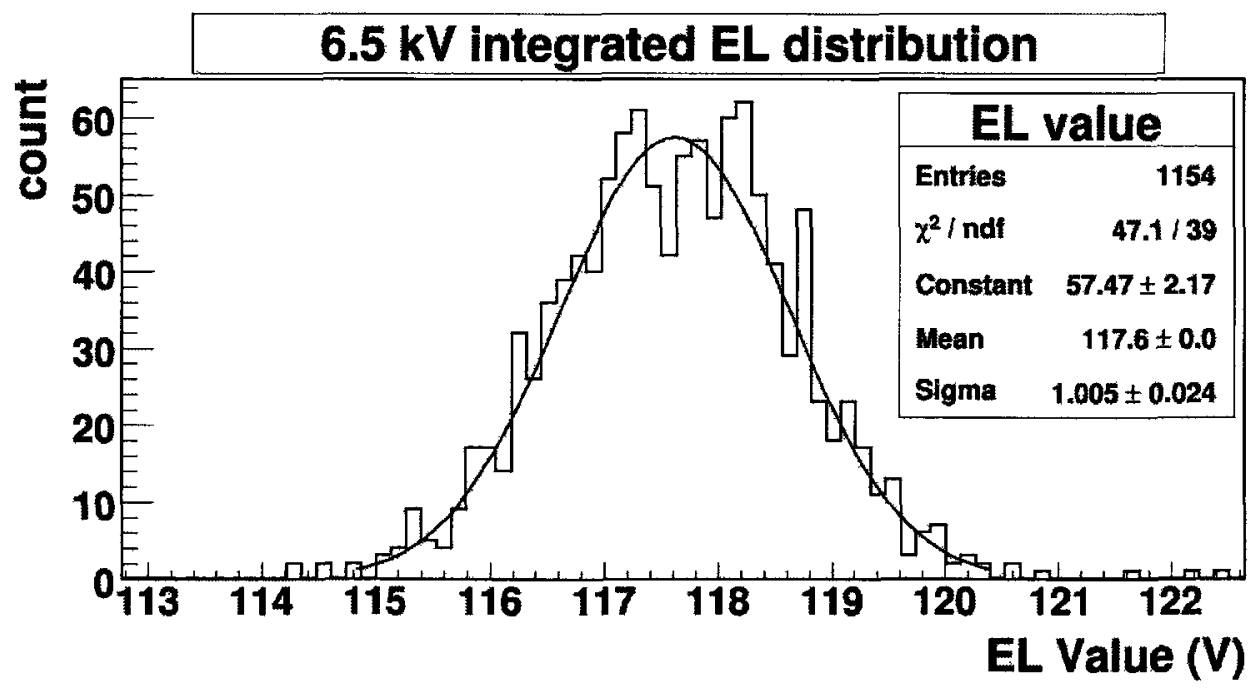

FIGURE 5.40: $6.5 \mathrm{kV}$ : distribution of integrated EL values. 
The EL Gaussian mean as a function of voltage, for a selection of angular ranges is given in Figure 5.41. The EL amplitude is essentially zero for voltages below threshold, and rises sharply above. The EL value obtained at $7.5 \mathrm{kV}$ is not consistent with overall trend in EL values, being lower then expected. The cause of this is likely due to $\alpha$ particles entering the EL region reducing the EL light yield. Any electrons that are produced in the EL region do not drift through the entire region, thereby reducing the light output compared to electrons produced outside the region. The plot exhibits a small dependence of the EL value on $\theta$. As the voltage increases so does the difference between EL values at a particular voltage. The amount of light loss for a parallel track relative to a perpendicular track can be estimated from the slope of the graph of the EL value versus onset time and a correction applied to remove the dependence (see Figure 5.11a). A linear fit was applied, excluding the low energy tail due to range outs, to the distribution obtained at each voltage and the slope determined. The obtained slopes are only reliable for voltages above $1.5 \mathrm{kV}$ and and no corrections were applied below this voltage. Using the obtained fit parameters and the time interval corresponding to each angular region, a correction is applied for each $\theta$-cut. Figure 5.42 plots EL values after the ad-hoc correction is applied. There a number of effects which account for the decrease in EL amplitude with voltage. The main contributors are the efficiency of the EL region increases with voltage due to the increase in EL radius, $\alpha$ tracks with a large $\theta$ have a lower efficiency then small $\theta$ tracks due to 
solid angle, and charge loss due to capture by electronegative impurities increases for longer drift times (large $\theta$ ).

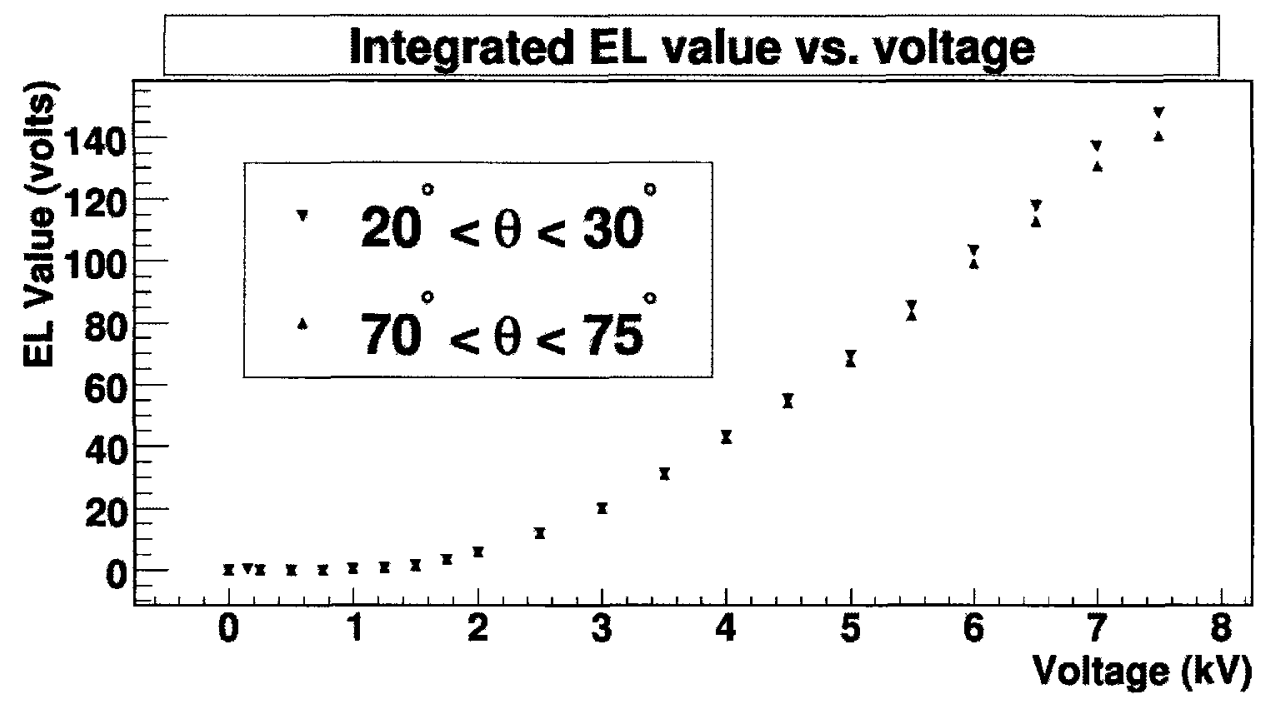

FIGURE 5.41: EL values vs. voltage corresponding to the given angular ranges. Statistical errors are included but are not visible on the given scale.

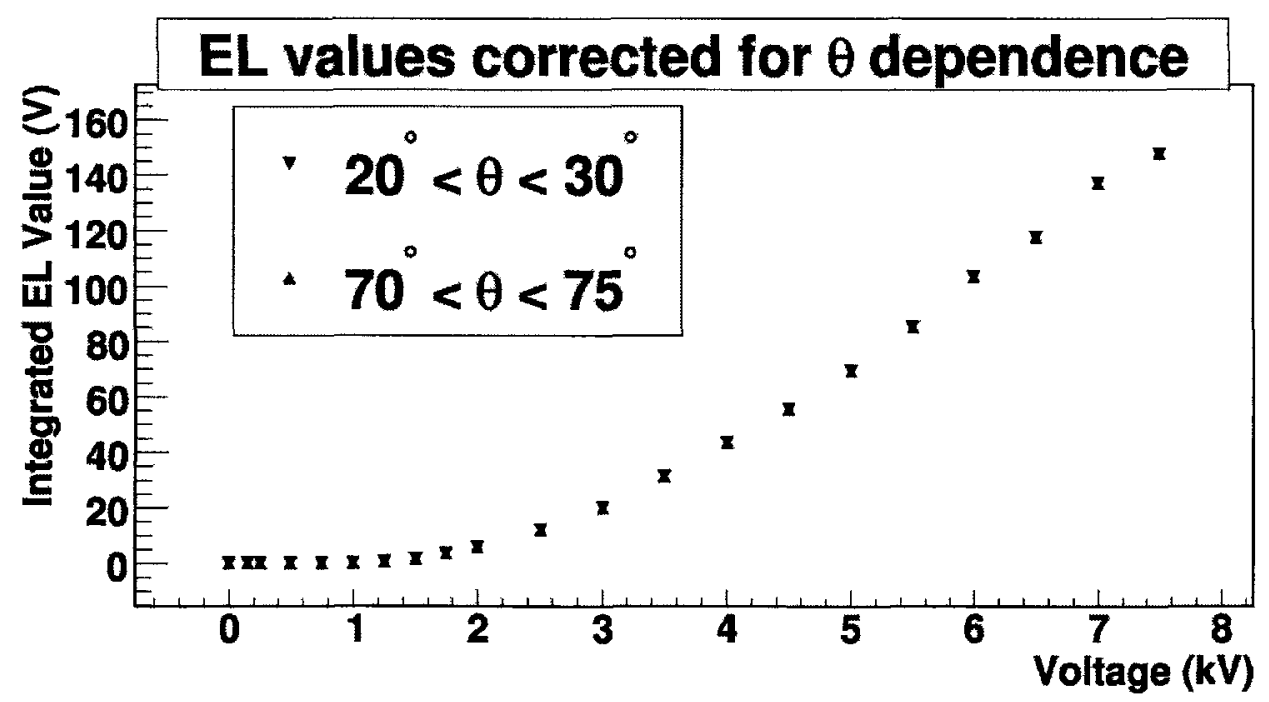

FIGURE 5.42: EL values vs. voltage for a selection of angular ranges with the angular dependence removed. Statistical errors are included but are not visible on the given scale. 
The EL optical gain is obtained from the ratio of the EL signal to the scintillation signal by applying $\mathrm{W}$-value corrections and efficiency averaging as described in Section 3. The efficiencies were obtained from the simulation, described in Section 4.1. Temperature corrections are not applied in making gain measurements since both the scintillation and EL signals track the same oscillation with a similar percent variation. Therefore any effects due to temperature induced variation should be essentially divided out. Figure 5.43 is the event by event EL gain corresponding to $6 \mathrm{kV}$. The distribution does not exhibit an appreciable variation, and the slope resulting from a linear fit is consistent with a straight line.

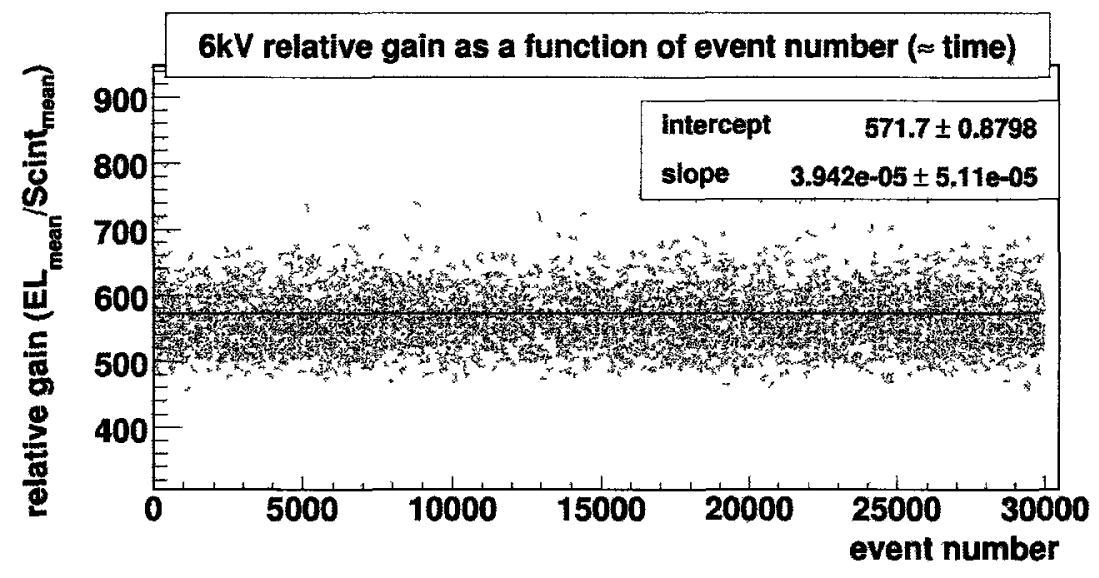

FIGURE 5.43: Event by event EL gain vs. event number: Temperature induced signal variations are cancelled out.

The following EL gain results were obtained using the average scintillation value over the voltage range (0-5) $\mathrm{kV}$ for reasons discussed in Section 5.6. The $\mathrm{W}$ value correction is highly dependent on the choice of $W_{s}$. The ionization $\mathrm{W}$-value is well established and consistent throughout the literature $\left(W_{\text {zon }}=21.9 \pm 0.4\right.$ 
eV) [27]. The results of the scintillation analysis indicates $W_{s}=47.2 \pm 0.7 \mathrm{eV}$, supported by the results of the GEANT4 simulation, where the assumption of $W_{s}=49.6$ resulted in a 3 standard deviation agreement with the mean number of scintillation photons obtained from the ADMCA calibration. After the corrections are applied the optical gain is fit with Equation (3.4), and the EL amplification and threshold parameters are determined for each angular range. The fit was performed over the voltage range (0.1-7.0) $\mathrm{kV}$. The point at $7.5 \mathrm{kV}$ was excluded since this point is thought to suffer from a reduced amplitude due to $\alpha$ particles entering the EL region. Figure 5.44 compares the EL gain corresponding to the two $\mathrm{W}$-values. Subsequent gain results will be given for $W_{s}=49.6 \pm 1.1 \mathrm{eV}$, since this value appears more consistent with the measured value from the detector.

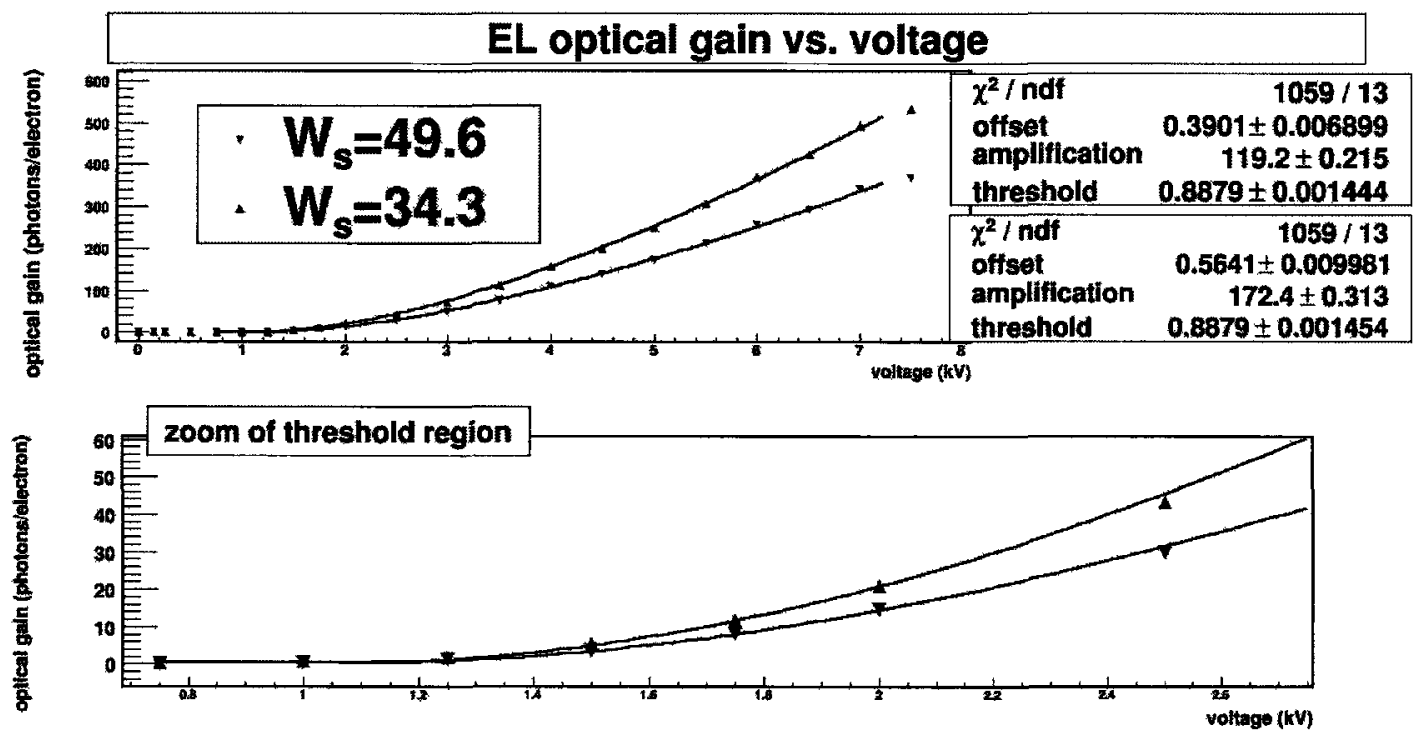

FIGURE 5.44: EL optical gain $\left(45^{\circ}<\theta<50^{\circ}\right)$ : Comparison of W-value corrections without efficiency averaging. Statistical errors are included but not visible on the given scale. 
Optical gain curves for the two extreme angular ranges are given in Figure 5.45. There is an angular dependence, with a number of contributing factors. The ratio of the amount of scintillation to EL light collected is dependent on the angular range, and the efficiency ratio in Equation 3.9 decreases with increasing $\theta$ and/or voltage, and therefore the EL gain measurements become somewhat skewed with angular range. Figure 5.46 gives the EL amplification and threshold parameters obtained for $W_{s}=49.6 \mathrm{eV}$ as a function of $\theta$-cut number. The values for the amplification and threshold parameters obtained from the 10th angular range $\left(45^{\circ}<\theta<50^{\circ}\right)$ are the most accurate since the efficiency for light collection in this range is close to unity, and therefore there is no skewing due to different EL ans scintillating light collection efficiencies. There is correlation between neighbouring points due to a small amount of overlap in the chosen angular ranges. The ranges were chosen with the requirement of keeping at least 1000 events. The values obtained for the 10th angular range are: amplification $=\mathrm{A}=119.9 \pm 0.280$ (stat) (photons $/ \mathrm{kV}$ ), threshold $=\mathrm{B}=0.882 \pm 0.0017$ (stat) $(\mathrm{kV} / \mathrm{cm}$-bar).

The $\mathrm{W}$-value correction assumes no loss to electronegative capture, and therefore $\approx 250000$ ionization electrons per event are expected $\left(E_{\alpha} / W_{i o n}=250230 \pm\right.$ 4488). The anode analysis results in a total number of collected ionization electrons on the order of $227900 \pm 1654$, resulting in a $\approx 9 \%$ overall loss. An independent measurement of the number of ionization electrons was made using the ADMCA setup, resulting in $229000 \pm 3690$ electrons, in agreement with the number obtained 


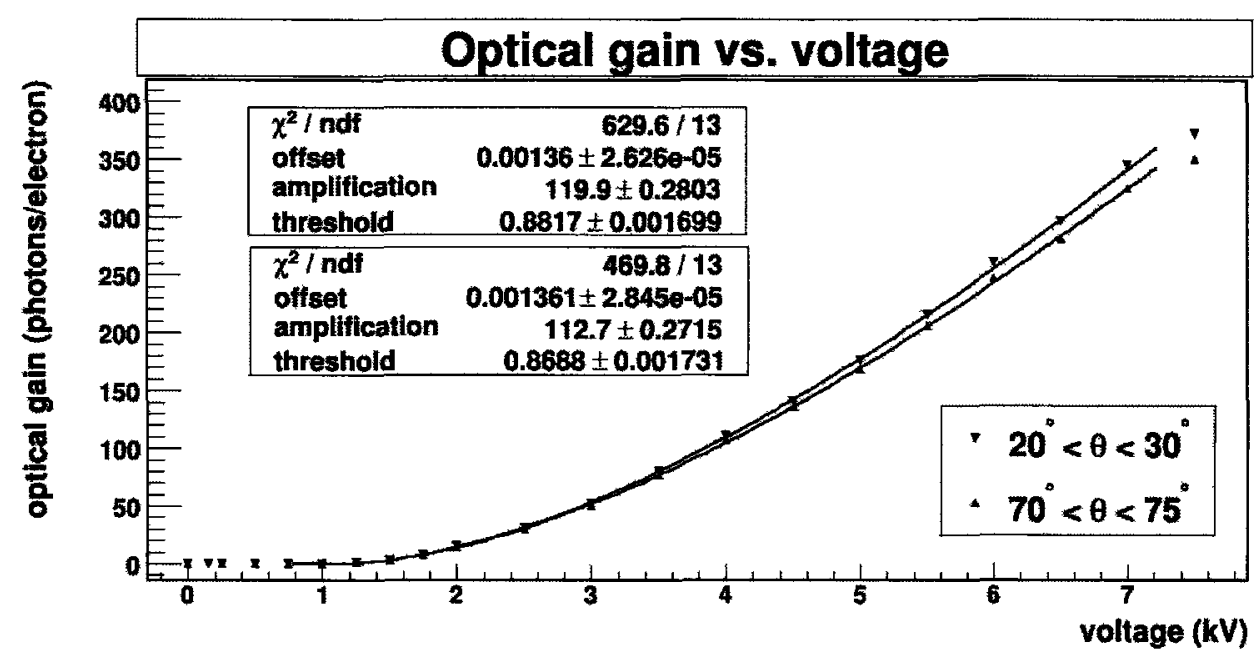

FIGURE 5.45: $W_{s}=49.6 \mathrm{eV}$ with efficiency averaging: Gain curves corresponding to the first and last $\theta$-ranges. Statistical errors are included but not visible on the given scale.
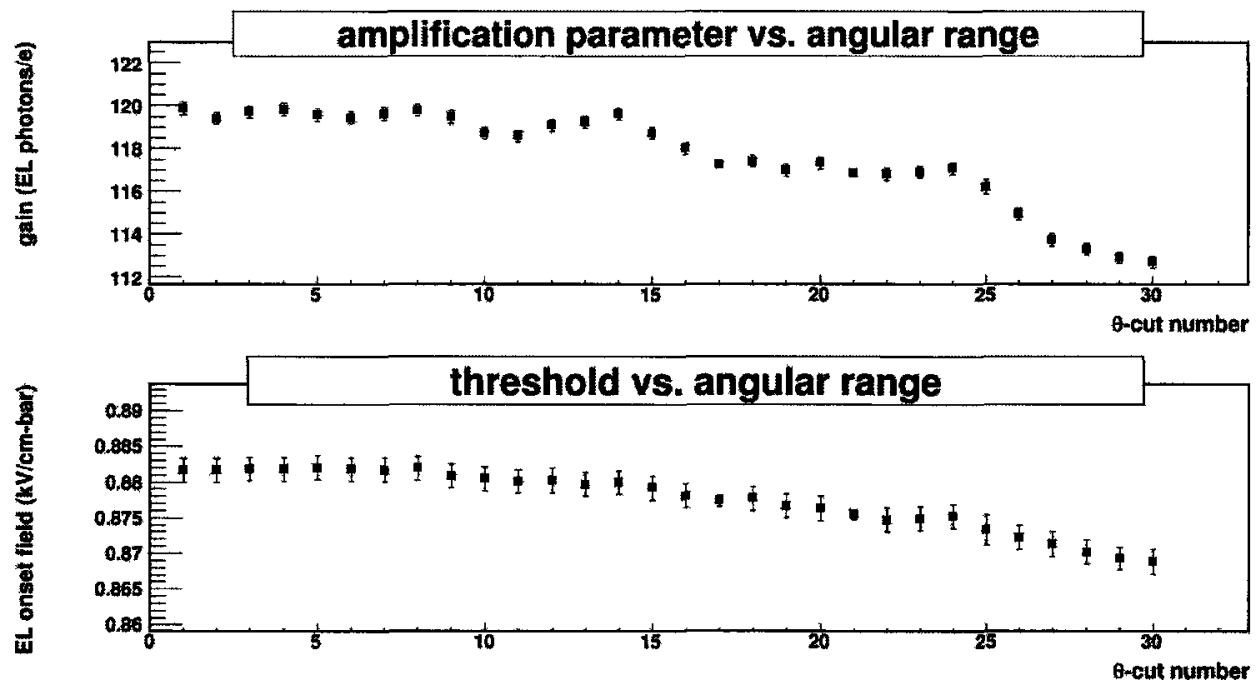

FIGURE 5.46: $W_{s}=49.6 \mathrm{eV}$ : EL amplification and threshold parameters vs. angular range $(\theta$-cut). The presented errors are statistical but there is correlation between neighbouring points due to overlap in the angular ranges.

from the digitized data. Therefore the measured EL gain is likely systematically lower due to the $9 \%$ difference in the number of ionization electrons. 
The statistical error for the gain was obtained assuming no correlation between the EL and scintillation signals. A plot of the EL value versus scintillation value obtained at $6 \mathrm{kV}$ is given in Figure 5.47, the plot exhibits no obvious correlation between the two variables and results in a correlation coefficient of 0.02 , supporting the assumption.

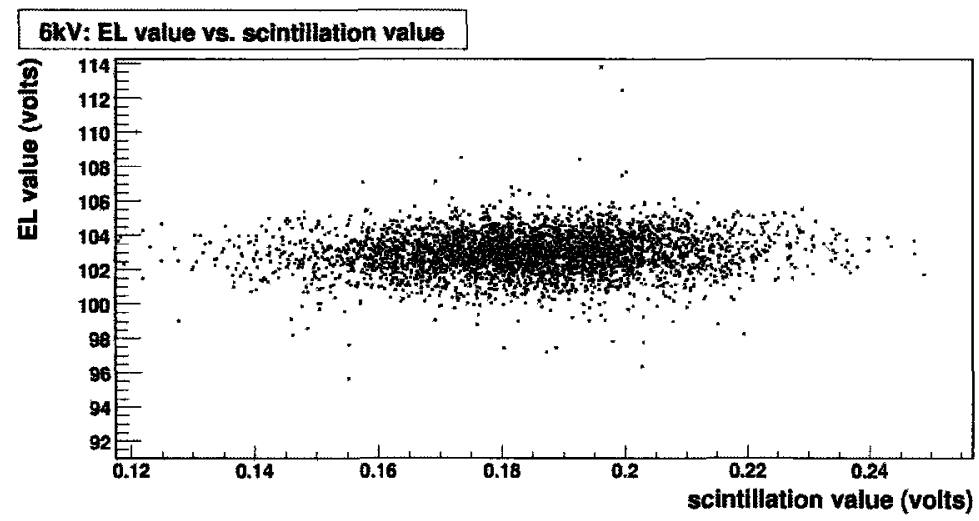

Figure 5.47: EL value vs. scintillation value.

The gain plots given so far include only the statistical errors but not the error introduced by the $\mathrm{W}$-value and overall electron loss corrections and efficiency averages. The gain plot for $W_{s}=49.6 \pm 1.1 \mathrm{eV}$ including systematic errors is given in Figure 5.48, where the fit was performed to the data including the statistical error, with the systematic error represented by the extended error bars. Systematic errors were estimated from the errors introduced by the $\mathrm{W}$-value and efficiency scaling and the choice of scintillation value. The values of A and B for the 10 angular range including systematic errors are: $A=119.9 \pm 0.280 \pm 8.7$ (sys) (photons $/ \mathrm{kV}$ ), $\mathrm{B}=0.882 \pm 0.0017$ (stat) \pm 0.0206 (sys) $(\mathrm{kV} / \mathrm{cm}$-bar). 


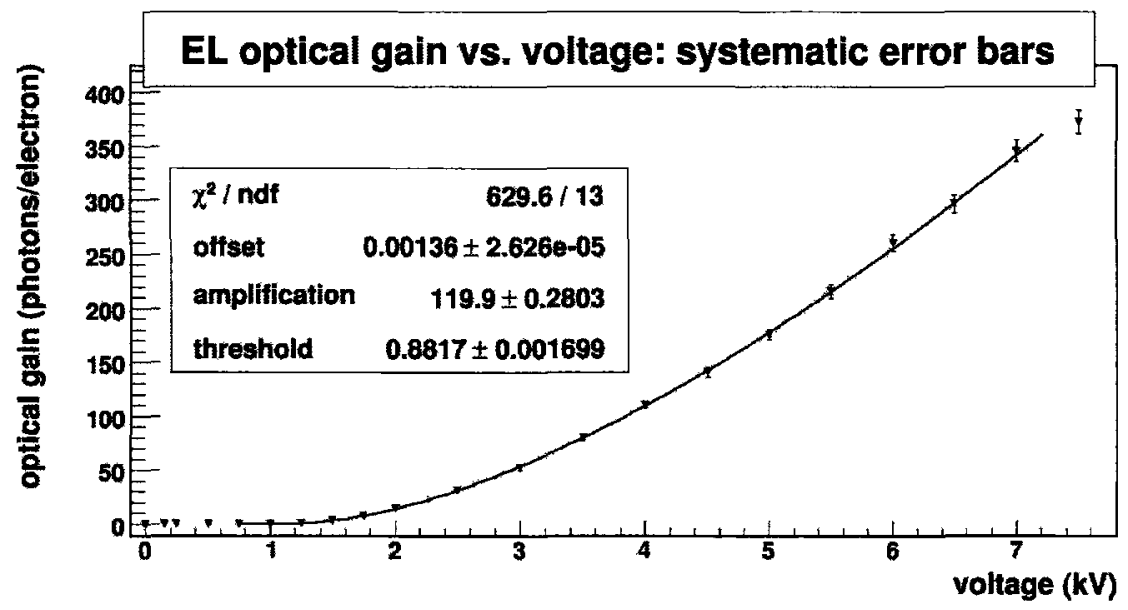

FIgURE 5.48: $W_{s}=49.6 \mathrm{eV}:\left(45^{\circ}<\theta<50^{\circ}\right)$ : EL optical gain including systematic errors introduced by the $\mathrm{W}$-value and efficiency scaling.

\subsubsection{EL resolution}

The resolution of the EL signal is determined from the distribution of integrated EL values as $\delta E / E=\sigma_{E L} /$ mean $_{E L}$. Temperature variations can degrade the resolution, but can be corrected out to allow for measurements of the detector resolution without temperature effects. The temperature corrections of Section 5.3 were applied. The EL value distributions obtained corresponding to the corrected and uncorrected EL signals are given in Figure 5.49. The Gaussian means are equivalent but the standard deviation of the temperature corrected distribution is $24 \%$ smaller then for the uncorrected distribution resulting in a $16 \%$ percent improvement in the resolution. 


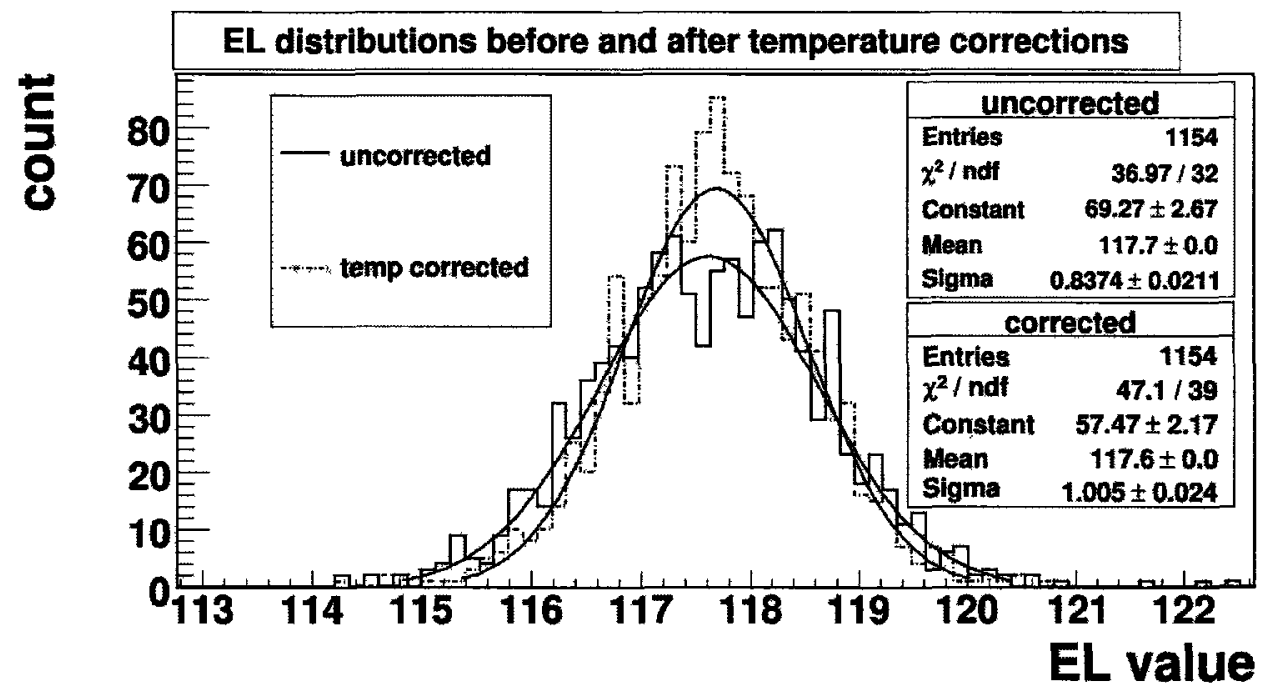

FIGURE 5.49: Temperature corrected and uncorrected EL distributions obtained at $6.5 \mathrm{kV}\left(20^{\circ}<\theta<30^{\circ}\right)$.

The resolutions obtained before and after temperature correction as a function of voltage, corresponding to the angular range $\left(\theta<30^{\circ}\right)$, are given in Figure 5.50. Both curves exhibit a sharp decrease in resolution over the voltage range (1.75-4.5) $\mathrm{kV}$, after which the resolution becomes relatively constant and stable with values on the order of $0.75 \%$ and $1 \%$ for the temperature corrected and uncorrected measurements respectively. There is a significant improvement in the resolution measurements over the entire voltage range for the temperature corrected signal. Also shown on the plot is the resolution expected from counting statistics. If one assumes that the resolution is constrained solely by photon counting statistics, then the expected resolution is determined in terms of the number of detected EL photons. Equation (3.4) is used to determine the expected number 
of EL photons per electron produced at a particular voltage. The expected number of photons per electron, combined with the average efficiency of the EL region $\left(E L_{e f f}\right)$, the ionization $\mathrm{W}$-value $\left(W_{i o n}=21.9 \mathrm{eV}\right)$, and the $\alpha$ particle energy $\left(E_{\alpha}\right)$, are used to obtain the expected number of EL photons $\left(N_{E L}\right)$. The resolution can then be represented as,

$$
\text { resolution }(\%)=\frac{1}{\sqrt{N_{E L}}} \times 100 \%=\frac{1}{\sqrt{E L_{e f f} \times \int N_{E L p h} \times E_{\alpha} / W_{i o n}}} .
$$

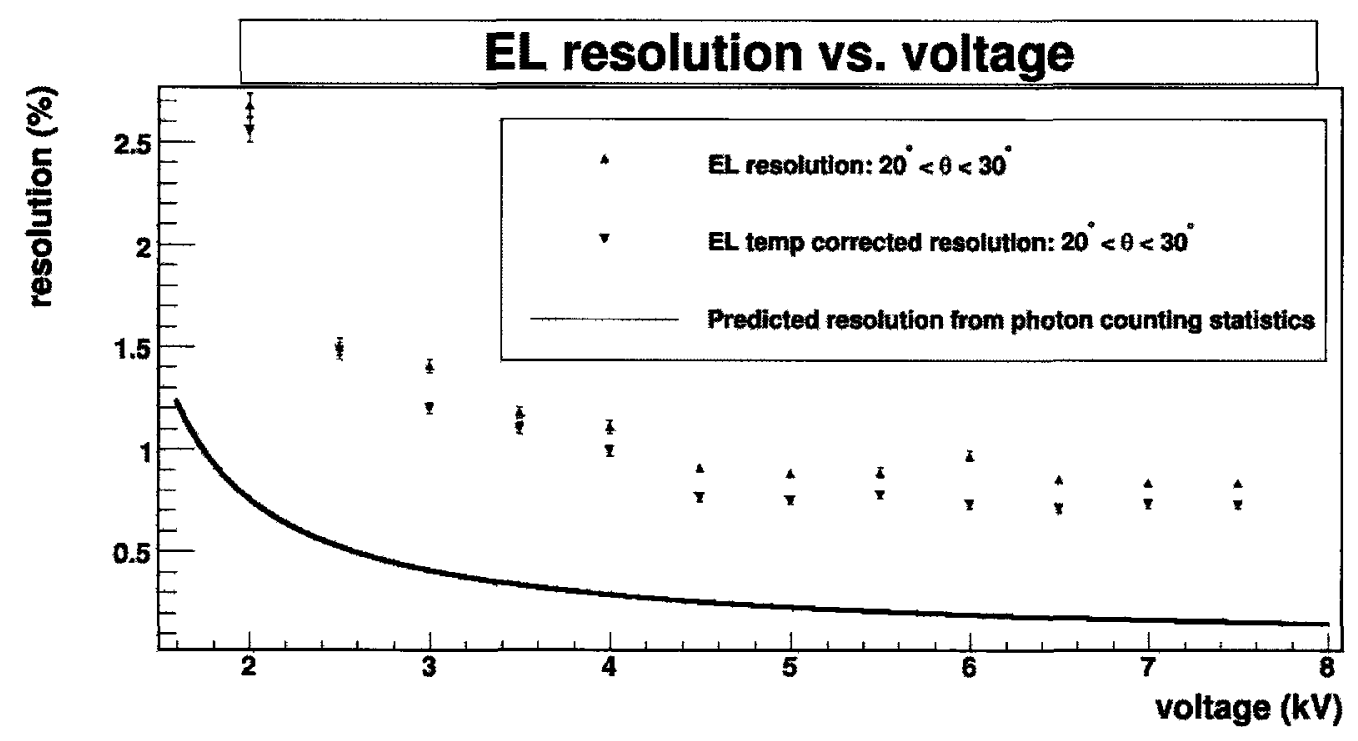

FIGURE 5.50: EL resolution for temperature corrected and uncorrected signals compared to the resolution expected from counting statistics.

The measured resolutions do not reach the values predicted from counting statistics alone. However, this is not surprising since there are obviously many 
factors other than counting statistics which affect the achievable resolution. The corrected resolution over the voltage range (4.5-7.5) $\mathrm{kV}$ is given in Figure 5.52, with the lowest value obtained at a voltage of $6.5 \mathrm{kV}$, resulting in a $0.71 \pm 0.02 \%$ resolution.

The values presented so far have been for the first angular range $\left(20^{\circ}<\right.$ $\theta<30^{\circ}$ ). The EL resolution slowly degrades as a function of the $\theta$. Figure 5.51 gives the EL resolution versus $\theta$-cut number, for voltages of 2,4 , and 6 $\mathrm{kV}$. The distributions obtained for the first $10 \theta$-cuts result in less than a $10 \%$ variation in measured resolutions. After the 10th cut the EL distributions become less Gaussian exhibiting low energy tails. The factors which contribute to the degradation of resolution with increasing $\theta$ have previously been discussed.

The EL energy resolution achieved in this work is on the order of $0.8 \%$ for the voltage range (4.5-7.5) $\mathrm{kV}$, and the angular range $\approx\left(20^{\circ}-38^{\circ}\right)$. This is an excellent resolution and a very encouraging result when considering the development of a xenon based TPC designed to achieve an energy resolution of $1 \%$ or better. The resolution in this work was obtained for a $5.49 \mathrm{MeV} \alpha$ particle source. The expected energy released in the $\beta \beta_{0 \nu}$ decay of ${ }^{136} \mathrm{Xe}$ is $2.48 \mathrm{MeV}$. To obtain the predicted energy resolution at the $\beta \beta_{0 \nu}$ Q-value, a $1 / \sqrt{E}$ extrapolation is used. There are a number of ways to parametrize the energy resolution of a detector, it is assumed here that the resolution is given by [51], 

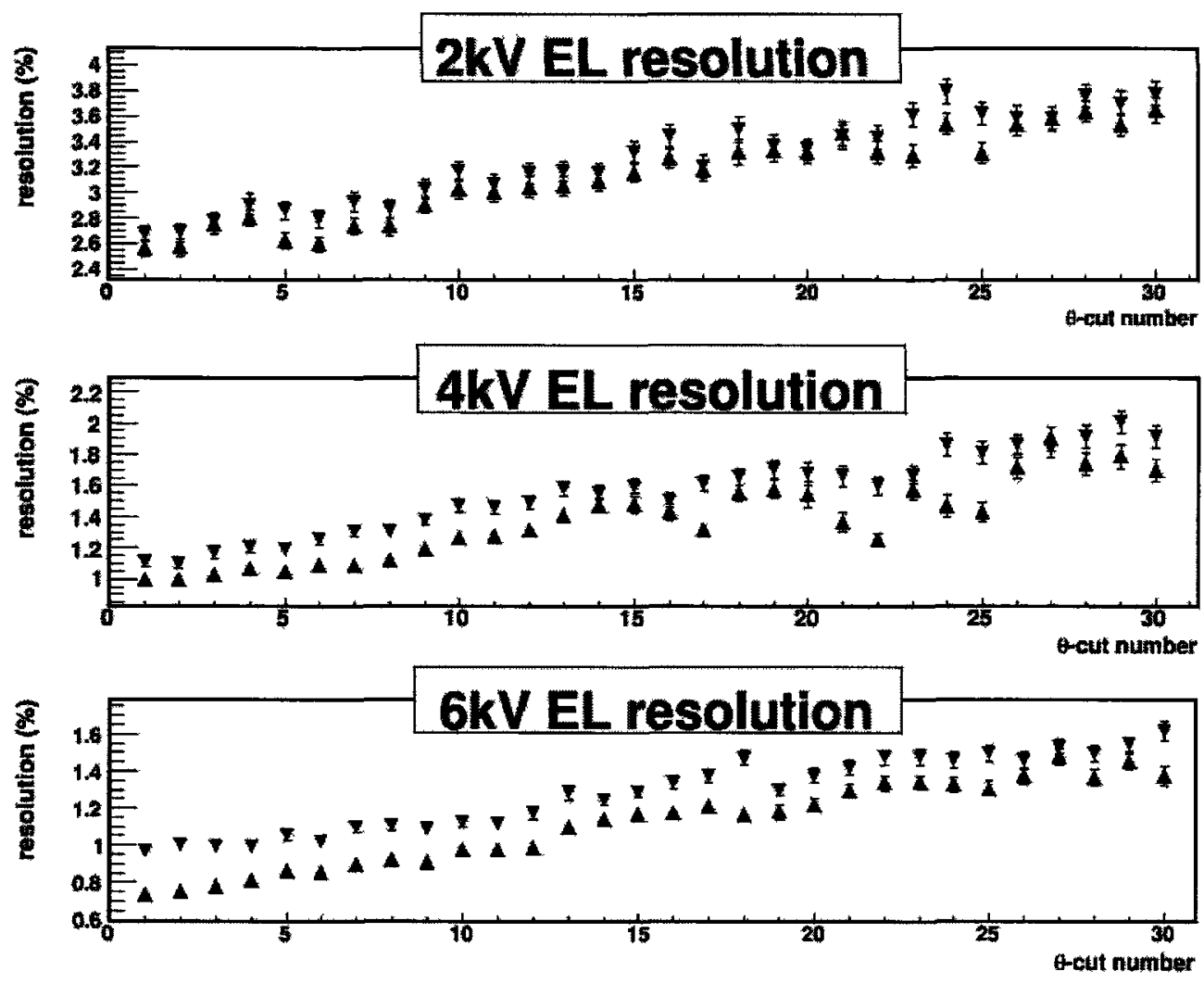

FIgURE 5.51: Temperature corrected EL resolution vs. $\theta$-cut for the selected voltages 2,4 , and $6 \mathrm{kV} . \triangle$ : temp corrected, $\nabla:$ uncorrected. The presented errors are statistical but there is correlation between neighbouring points due to overlap in the angular ranges.

$$
\frac{\sigma_{E}}{E}=A \oplus \frac{B}{\sqrt{E}}
$$

Here $\mathrm{A}$ and $\mathrm{B}$ are constants, with $\mathrm{A}$ being a noise term and $\mathrm{B}$ a statistical term.

Due to only having one energy data point both constants cannot be determined and an assumption of $A=0$ is used. This assumes that the resolution is dominated by the statistical term and that there is no contribution from noise; which is not 
completely realistic but also not unreasonable, in reality $0<A<B$.. Taking a value of $0.8 \%$ as the available energy resolution for $5.49 \mathrm{MeV} \alpha$ particles in xenon, the expected resolution at the $\beta \beta_{0 \nu}$ Q-value is obtained from the extrapolation. Figure 5.53 is the resulting resolution curve, resulting in a resolution of $\delta(E) / E=$ $1.18 \%$ at the Q-value.

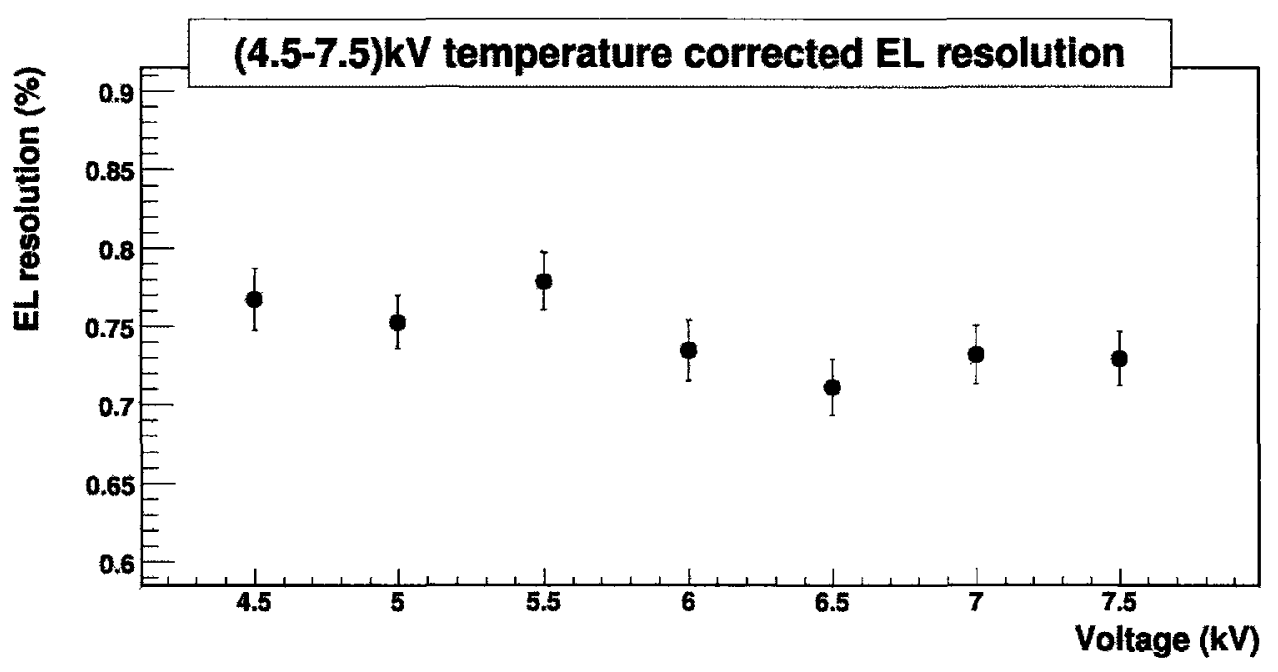

FIGURE 5.52: Temperature corrected EL resolutions for the voltage range (4.57.5) $\mathrm{kV}$. 


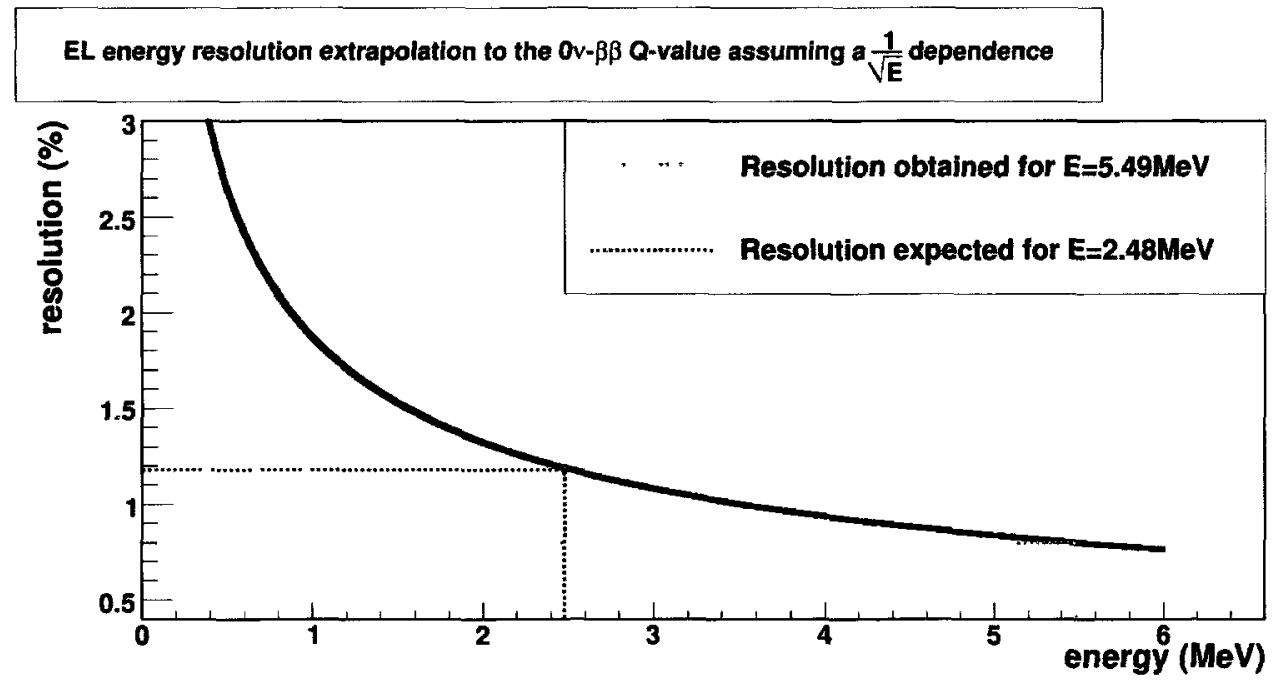

FIGURE 5.53: Energy resolution extrapolation curve obtained by taking $0.8 \%$ as the energy resolution at $5.49 \mathrm{MeV}$. 


\section{Chapter 6}

\section{Discussion and Conclusions}

General results have been presented for ionization, scintillation, and EL signals produced by alpha particles in 1 bar of xenon obtained from the cylindrical EL test chamber described in Chapter 3. The main physics measurements and their associated errors will now be addressed.

The ionization signal was obtained from the distribution of anode amplitudes. Corrections for angular dependence and preamp decay time were applied. The total number of collected ionization electrons was measured to be $227900 \pm 1654$ assuming $W_{\text {ron }}=21.9 \pm 0.4 \mathrm{eV}$. The value was obtained from a constant fit applied to the voltage range (1.5-7.50) $\mathrm{kV}$ (see Section 5.5). This value is $9.1 \pm 0.2 \%$ lower than the expected number indicating an appreciable energy/charge loss. An independent measurement made using the ADMCA setup 
resulted in $229000 \pm 3690$ electrons, in agreement with the number obtained from the digitized data. This loss is likely due to electronegative capture during the drift time. This amount of loss is somewhat surprising since the xenon used has a 99.995\% purity and was passed through a heated zirconium getter during filling. All components were cleaned before being introduced to the chamber. The final chamber vacuum pressure before the fill was $6.2 \times 10^{-6} \mathrm{mb}$ and the chamber was leak checked using a helium leak detector resulting in the detection of no significant leaks. The most reasonable explanation for the cause of the xenon contamination is outgassing from various components in the detector such as the peek support posts used to hold the anode in place and the Teflon sleeves and end-cap used to prevent electrical breakdown. Another factor which can contribute to the loss is contamination on the surface of the source such as dust, dirt, or oil from human hands due to mishandling. Also, the source is old and the exact handling history is not well known. Drift velocity measurements are sensitive to gas contamination and a comparison to the expected values has been used to indicate gas contamination in previous xenon fills. The calculations of drift velocity corresponding to the xenon used for the current measurements, are in good agreement with the simulation results, indicating any contaminants present were not significant enough to alter the transport properties within the errors on the measurements.

The resolution of the ionization signal was determined from the uncorrected anode amplitude distributions from $\delta E / E=\sigma_{\text {anodeamp }} / M$ ean anodeamp. The 
measured resolution corresponding to the first $\theta$-cut (see Figure 5.30) reaches a stable value on the order of $1.3 \%$ over the voltage range (3-6) $\mathrm{kV}$. The resolution becomes poor above $6 \mathrm{kV}$ and there is an outlier point at $3 \mathrm{kV}$ which does not quite follow the behaviour of the resolution curve. Nothing out of the ordinary is apparent in examining the amplitude distribution for $3 \mathrm{kV}$ except that the width is larger. The measured resolutions exhibited an angular dependence which could not be removed with angular corrections for varying drift distances, and is due to energy loss mechanisms which are dependent on angle of emission. A noticeable amount of noise was present on the raw signal making precise measurements of the signal amplitude difficult, introducing a significant variation in the width of the distributions. The preamp used was fairly old and had been modified a number of times. Efforts were made to reduce the noise but were unsuccessful. The detector was optimized for measurements of the light signal and measurements of the anode signal were of secondary importance and therefore these issues were not of paramount concern. Systematic errors were not evaluated for the anode signal analysis.

The scintillation analysis resulted in scintillation values which exhibited a dependence on $\theta$. The dependence is attributed to a combination of the choice of integration time window and the dependence of EL onset time on the angle of $\alpha$ emission. Two integration windows were chosen (300 ns and $600 \mathrm{~ns}$ ) and it was found that the $300 \mathrm{~ns}$ window resulted in a mean scintillation value which agrees 
within $4 \%$ of the $600 \mathrm{~ns}$ window for voltages below $4 \mathrm{kV}$, exhibiting a smaller angular dependence above $5 \mathrm{kV}$. The final scintillation value chosen for the gain measurements was the average scintillation value taken over the voltage range $(0-5) \mathrm{kV}$ for the $600 \mathrm{~ns}$ time window based on the results of Section 5.6.

The results from the independent ADMCA scintillation analysis indicated 434.2 \pm 6.4 photoelectrons per $\alpha$ ionization event. Using the measured number of photoelectrons and the average scintillation region efficiency obtained from the simulation, the scintillation $\mathrm{W}$-value was estimated to be $W_{s}=47.2 \pm 0.7 \mathrm{eV}$, which is in better agreement with the larger value given in the literature $\left(W_{s}=\right.$ $49.6 \pm 1.6 \mathrm{eV})$. This is by no means an accurate determination of the $W_{s}$ value, and only is used as a consistency check. The results of the GEANT4 $\alpha$ particle simulation predicted the mean numbers of detected photons to be $416.8 \pm 22.04$ and $573 \pm 26.8$ for $W_{s}=49.6 \mathrm{eV}$ and $W_{s}=34.3 \mathrm{eV}$ respectively. The number obtained for $W_{s}=49.6 \mathrm{eV}$ is in agreement with the measured number from the ADMCA calibration, supporting the use of the larger of the two W-values in EL gain measurements. The measured resolution of the scintillation signal is fairly stable and is on the order of $7 \%$ over the voltage range (0-5) $\mathrm{kV}$ (see Figure 5.35), with the lowest measured resolution being $5.76 \pm 0.14 \%$. The resolution degrades sharply after $\approx 5.5 \mathrm{kV}$. The best resolutions are obtained for the first two or three angular regions, after which point the resolution slowly degrades with increasing $\theta$. Temperature corrections applied to the scintillation signal did not result in 
a significant increase to the measured resolutions as was expected following the results of Section 5.3.

The integrated EL amplitudes were obtained over the voltage range (0.07.5) $\mathrm{kV}$. The measurements resulted in EL values consistent with 0 for voltages below $1 \mathrm{kV}$, after which EL generation begins and there is a sudden rise in value which becomes basically linear above $3 \mathrm{kV}$. Temperature corrections were not applied to the EL values as they would distort the real distribution mean. There is an angular dependence of the EL value which was corrected for using an ad-hoc correction. The EL value corresponding to $7.5 \mathrm{kV}$ does not follow the trend in the data and has a lower value then expected. This lower value is attributed to $\alpha$ particles entering the EL region, resulting in a smaller EL yield. Using Equation (3.2) and the EL threshold parameter obtained from the fit gives an EL radius of $3.64 \mathrm{~cm}$ at $7.5 \mathrm{kV}$. The expected $\alpha$ range is $2.4 \mathrm{~cm}$ supporting the assumption that near perpendicular ionization tracks are entering the EL region for $7.5 \mathrm{kV}$.

The EL optical gain was measured from the ratio of the Gaussian means obtained for the EL and scintillation distributions by applying efficiency averaging and $\mathrm{W}$-value corrections. The results of the ADMCA scintillation analysis and from the simulation indicate the use of $W_{s}=49.6 \pm 1.1 \mathrm{eV}$ as the appropriate value for gain measurements. The optical gain curve corrected using $W_{s}=49.6 \mathrm{eV}$ was then fit with Equation (3.4), and the EL amplification and threshold parameters 
are obtained as a function of $\theta$. The EL gain exhibited an angular dependence which was not removed by the efficiency averaging. A rough systematic error analysis was applied, where the main contributors to the error on the gain measurements were assumed to be errors introduced by the efficiency averaging and the associated error on the W-values. The resulting EL gain parameters corresponding to the angular range, $\left(45^{\circ}<\theta<50^{\circ}\right)$, are $A=119.9 \pm 0.280($ stat $) \pm 8.7($ sys $)$ (photons $/ \mathrm{kV})$ and $B=0.882 \pm 0.0017($ stat $) \pm 0.0206($ sys $)(\mathrm{kV} / \mathrm{cm}-\mathrm{bar})$. The values are presented for this angular range because the efficiency ratio is one and therefore the measurement is not skewed due to a mismatch in the light collection efficiency for photons generated in the EL and scintillation regions.

The resolution of the EL signal was obtained as a function of applied voltage and angular range. Temperature corrections were applied to the EL signal resulting in improvements ranging from (10-20)\% in the measured resolutions. The resolution over the voltage range (4.5-7.5) $\mathrm{kV}$ becomes relatively stable on the order of $0.75 \%$, reaching a minimum value of $0.71 \pm 0.02 \%$, obtained at 6.5 $\mathrm{kV}$. The uncorrected values follow the same trend over the voltage range but they level off on the order of $0.9 \%$ with a minimum resolution of $0.85 \pm 0.02 \%$. There are two main sources of systematic error introduced in the measurements of the EL resolution, one due to the choice of integration time window and the second from the choice of baseline used in the integration. The associated systematic 
errors are estimated from the difference in the mean and $\sigma$ of the EL distributions resulting from the different variables. The first integration window started at the scintillation end time and extended to the trace end time. The second time window was obtained from the simulation of drift times, and was used to determine the results given. The difference in the mean and $\sigma$ of the resulting EL distributions was adopted as the systematic error on the measurement. The two errors are assumed to be uncorrelated and are added in quadrature. Figure 6.1 plots the resolution including the associated systematic error corresponding to the angular range $\left(20^{\circ}<\theta<30^{\circ}\right)$. The errors are large for low voltages and decrease with increasing voltage. For the resolution at $6.5 \mathrm{kV}$ is then given as $0.71 \pm$ (stat) $\pm 0.02($ sys $) \%$.

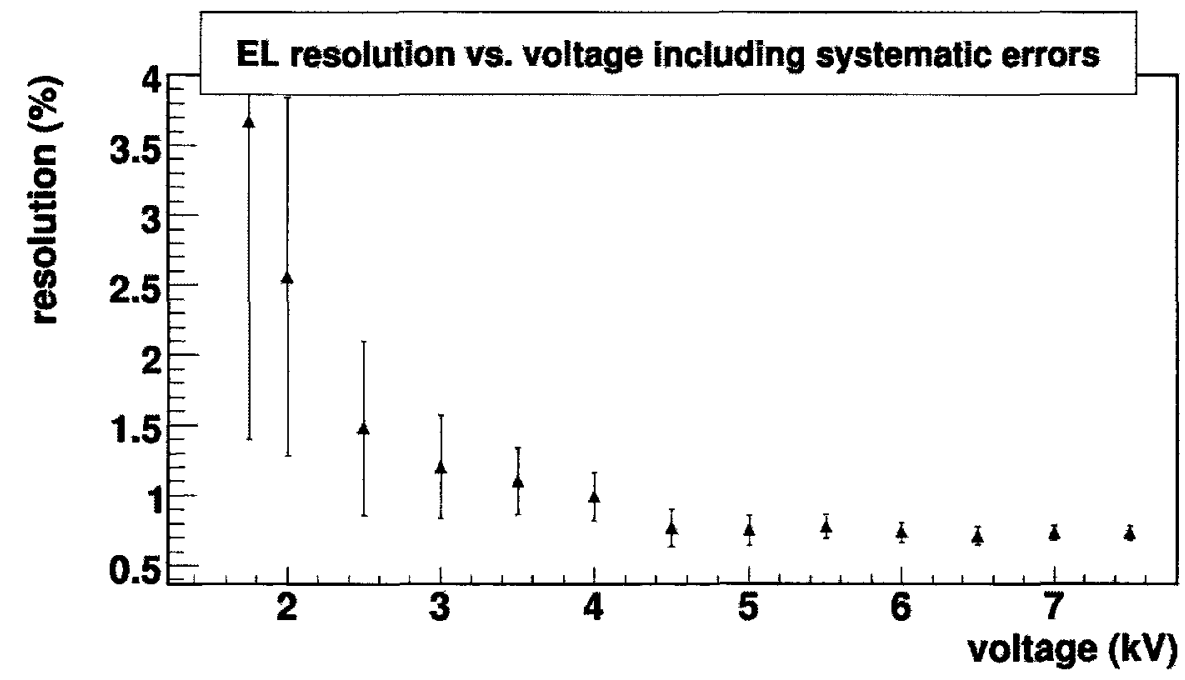

FIGURE 6.1: EL resolution as a function of voltage including systematic errors due to integration windowing and baseline subtraction. 
The main results of the work are summarized as follows. The EL gain parameters were measured to be $A=125.6 \pm 0.28($ stat $) \pm 2.4861($ sys $)($ photons $/ k V)$ and $B=0.9185 \pm 0.0017$ (stat) \pm 0.01297 (sys) for the angular range $\left(20^{\circ}<\theta<\right.$ $30^{\circ}$, and an angular dependence was observed. The analysis of EL optical gain results in fit parameters which do not agree with the published values, where threshold parameters on the order of (7-10)\% larger, and amplification parameters which are on the order of (10-15)\% smaller than the recent published measurements, and the values predicted from Boltzmann calculations and MC studies. The detector's energy resolution was measured as a function of voltage and angle, and resolutions on the order of $0.8 \%$ were achieved with the best measured value being $0.71 \pm$ (stat) \pm 0.02 (sys) $\%$, an excellent result. By adopting $0.8 \%$ as the energy resolution of the detector corresponding to $5.49 \mathrm{MeV}$ of deposited energy, the expected resolution at the $\beta \beta_{0 \nu}$ Q-value was obtained by assuming that the resolution represented by Equation (5.4) is dominated by the statistical contribution with no noise contribution. The result of the extrapolation predicts an energy resolution of $1.18 \%$ at the $\beta \beta_{0 \nu}$ Q-value. This result implies that measurement of the $\beta \beta$ energies via EL production is a very promising approach to achieve the energy resolution requirements for a tonne scale experiment to reach a sensitivity to the neutrino mass spectrum down to a few $\mathrm{eV}$. 


\section{References}

[1] O. Cremonesi. Neutrinoless Double Beta Decay: Present and Future. hepex/0210007v1, pages 1-10, 2002.

[2] S. R. Elliott F.T. Avignone III and J. Engel. Double beta decay, Majorana neutrinos, and neutrino mass. Reviews of Modern Physics, 80:481-516, 2008.

[3] C.M.B. Monteiro, L.M.P. Fernandes, J.A.M. Lopes, L.C.C. Coelho, J.F.C.A. Veloso, J.M.F. dos Santos, K. Giboni, and E. Aprile. Secondary Scintillation Yield in Pure Xenon. Journal of Instrumentation, 2:5001-5012, 2007.

[4] Kurt Riesselmann. logbook: Neutrino Invention. Symmetry: A joint Fermilab/SLAC publication., 4:4-4, 2007.

[5] Karsten M. Heeger. Evidence For Neutrino Mass: A Decade Of Discovery. In Conference preceedings: arXiv:hep-ex/0412032v2, pages 1-16, 2008. 
[6] T. Araki et al. (KamLAND Collaboration. Measurement of Neutrino Oscillation with Kamland: Evidence of Spectral Distortion. Phys. Rev. Letters, 94: 801-806, 2005.

[7] Q. R. Ahmad et al (SNO Collaboration). Direct Evidence for Neutrino Flavor Tranformation From Neutral-Current Interactions in the Sudbury Neutrino Observatory. Phys. Rev. Lett., 89:001301-111306, 2002.

[8] S. N. Ahmed et al (SNO Collaboration). Measurement of the Total Active B Solar Neutrino Flux at the Sudbury Neutrino Observatory with Enhanced Neutral Current Sensitivity. Phys. Rev. Lett., 92:181301, 2004.

[9] S. Pascoli and S.T. Petcov. The absolute neutrino mass scale, neutrino mass spectrum, Majorana CP-violation and neutrinoless double-beta decay. $\mathrm{Nu}$ clear Physics B, 734:24-49, 2006.

[10] Donald H. Perkins. Introduction to High Energy Physics. Addison Wesley Publishing Conpany, Menlo Park, Ca, 1987.

[11] Y. Ashie et al Super-Kamiokande Collaboration. Measurement of atmospheric neutrino oscillation parameters by Super-Kamiokande i. Physical Review D, 71:112005-112040, 2005. 
[12] R. Wendell et al. (The Super-Kamiokande Collaboration). Atmospheric neutrino oscillation analysis with subleading effects in Super-Kamiokande i, ii, and iii. Phys. Rev. D, 81:1-6, 2010.

[13] J. Boger et al (SNO Collaboration). The Sudbury Neutrino Observatory. Nucl. Inst. Meth., A449:172, 2000.

[14] K. Eguchi et al (KamLAND Collaboration. Precision Measurement of Neutrino Oscillation Parameters with Kamland. Phys. Rev. Lett., 90:1, 2003.

[15] W.C. Haxton and Jr. G.J. Stephenson. Double beta decay. Prog. Part. Nucl. Phys., 12:409-479, 1984.

[16] S. F. King. Double beta decays and neutrino masses. Rep. Prog. Phys., 67: 107-157, 2003.

[17] H. V. Klapdor-Kleingrothaus et al. Evidence for Neutrinoless Double Beta Decay. Modern Physics Letters A, 16:2409-2420, 2001.

[18] S. R. Elliott and J. Engel. Double beta decay. Journal of Physics G: Nucl. and Part. Phys., 30:183-215, 2004.

[19] Vincent Lepeltier. Review on TPCs. Journal of Physics: Conference Series, $65: 1-13,2007$.

[20] F. Sauli and A. Sharma. Micropattern gaseous detectors. Ann. Rev. Nucl. Part. Sci., 49:341, 1999. 
[21] Y. Giomataris, P. Rebourgeard, J.P. Robert, and G. Charpak. Micromegas: a high-granularity position-sensitive gaseous detector for high particle-flux environments. Nucl. Inst. Meth. in Physics Research A, 376:29, 1996.

[22] F. Sauli. GEM: a new concept for electron amplification in gas detectors. Nucl. Inst. Meth. in Physics Research A, 386:531, 1997.

[23] J. B. Rajam. Atomic Physics. S. Chand and Co., New Delhi, 1960.

[24] David Nygren. High-pressure xenon gas electroluminescent TPC for 0-v double beta decay search. Nucl. Intr. and Meth. in Physics Research A, 603: $337-348,2009$.

[25] S.C.P. Leite M. Salete. Radiolulinescence of Rare Gases. Portgal. Phys, 11: $53-100,1980$.

[26] M. Mimura, S. Kobayashi, T. Ishikawa, M.Miyajima, and N. Hasebe. Intensity and time profile of recombination luminescence produced by an alpha-particle in dense xenon gas. Nucl. Inst. and Meth. in Physics Research A, 613:106-111, 2009.

[27] M. Mimura, S. Kobayashi, N. Masuyama, M. Miyajima, and N. Hasebe. Average Numbers of Scintillation Photons and Electrons Produced by Alpha Particle in High-Density Xenon Gas. Japan Society of Applied Physics, DOI:10.1143/JJAP.48.076501:076501-076508, 2009. 
[28] M. H. R. Hutchinson. Excimers and excimer lasers. Applied Physics, 21: 95-114, 1980.

[29] M. Suzuki and S. Kubota. Mechanism of Proportional Scintillation in Argon, Krypton and Xenon. Nucl. Inst. and Meth., 164:197-199, 1979.

[30] J. Wieser, D. E. Murnick, A. Ulrich, H. A. Huggins, A. Liddle, and W. L. Brown. Vacuum ultraviolet rare gas excimer light source. Rev. Sci. Instrum., 68:1360-1365, 1997.

[31] K. Saito, S. Sasaki, H. Tawara, T. Sanami, and E. Shibamura. Simultaneous Measurements of Absolute Numbers of Electrons and Scintillation Photons Produced by 5.49 Mev Alpha Particles in Rare Gases. IEEE Transactions on Nuclear Science, 50:2452-2459, 2003.

[32] M. Miyajima S. Sasaki and E. Shibamura. Absolute number of photons produced by alpha-particles in liquid and gaseous xenon. Nucl. Inst. Meth. in Physics Research B, 63:297-308, 1991.

[33] M. Alegria Feio and A.J.P.L. Policarpo. Secondary Light Emission by Nobel Gases. Nucl. Inst. and Meth., 217:473-478, 1983.

[34] M. Alegria Feio, A.J.P.L. Policarpo, and M.A.F. Alves. Thresholds for Secondary Light Emission by Noble Gases. JJAP, 21:1184-1190, 1982. 
[35] F.P. Santos, T.H.V.D. Dias, A.D. Stauffer, and C.A.N. Conde. Threedimensional Monte Carlo calculation of the VUV electroluminescence and other electron electron transport parameters in xenon. Journal of Physics D, 27:42-48, 1993.

[36] T.H.V.D. Dias, A.D. Stauffer, and C.A.N. Conde. A unidimensional Monte Carlo simulation of the electron drift velocities and electroluminescence in argon, krypton, and xenon. Journal of Physics D, 19:527-545, 1986.

[37] F. Favata, A. Smith, M. Badvaz, and T.Z. Kowalski. Light Yield as a Function of Gas Pressure and Electric Field in Gas Scintillation Proportional Counters. Nucl. Inst. and Meth. in Physics Research A, 294:595 601, 1990.

[38] C.A.N. Conde, L.R. Ferreira, M. Fatima, and A. Ferreira. The Secondary Scintillation Output of Xenon in a Uniform Field Gas Proportional Scintillation Counter. IEEE Trans. Nucl. Sci, 24:221-224, 1977.

[39] J. Seguinot, J. Tischhauser, and T. Ypsilantis. Liquid xenon scintillation: photon yield and fano factor measurements. Nucl. Inst. and Meth. in Physics Research A, 354:280-287, 1995.

[40] David Nygren. Optimal detectors for WIMP and 0-vBB searches: Identical high-pressure xenon gas TPCs? Nucl. Intr. and Meth., Phys. Research A, $581: 632-642,2007$. 
[41] M. Ball on Behalf of the NEXT collaboration. NEXT: A Neutrinoless Double Beta Decay Experiment. In IEEE Nuclear Science Symposium Conference Record, pages 1034-1039, 2009.

[42] A. Bolozdynya, V. Egorov, A Koutchenkov, G. Safronov, G. Smirnov, and S. Medved. A high pressure xenon self-triggered scintillation drift chamber with 3D sensitivity in the range of 20-140 kev deposited energy. Nucl. Inst. and Meth. in Physics Research A, 385:225-238, 1997.

[43] D.Yu. Akimov, A.A. Burenkov, V.N. Kuzichev, V.L. Morgunov, and V.N. Solovov. Cylindrical Scintillation Proportional Counter with WLS Fiber Readout. Nuclear Science, 45:497 - 501, 1998.

[44] http://www.nist.gov/. The National Institute of Standards and Technology (nist) is an agency of the u.s. commerce department.

[45] Ossama S. AbouZeid. Phys 4909 Final Report: Ionization Signal Measurement via Electroluminescence for the Exo Collaboration. 2009.

[46] W. M. Burton and B. A. Powell. Fluorescence of Tetraphenyl-Butadiene in the Vacuum Ultraviolet. APPLIED OPTICS, 12:87-90, 1973.

[47] J. Allison et al. Geant4 developments and applications. Nuclear Science, IEEE Transactions on, 53:270-278, 2006. 
[48] J. L. Pack, R. E. Voshall, A. V. Phelps, and L. E. Kline. Longnitudinal electron diffusion coefficients in gasses: Noble gases. J. Appl. Phys., 71:5363$5371,1992$.

[49] S. Ramo. Currents induced by electron motion. Proc. IRE, 27:584-585, 1939.

[50] R. DeVoe E. Conti, G. Gratta, T. Koffas, S. Waldman1, J. Wodin, G. Bower D. Akimov and, M. Breidenbach, R. Conley, M. Danilovand Z. Djurcic, A. Dolgolenko, C. Hall, A. Odian, A. Piepke, C.Y. Prescott, P.C. Rowson, K. Skarpaas, J-L. Vuilleumier, K.Wamba, and O. Zeldovich (EXO Collaboration). Correlated fluctuations between luminescence and ionization in liquid xenon. Phys. Rev B, 68:1-5, 2003.

[51] R. Fernow. Introduction to experimental particle physics. Cambridge University Press, New York, NY, 1989. 